Mechanical Regulation of Fibroblast Migration and

Collagen Remodeling in Healing Myocardial Infarcts

A Dissertation
Presented to
the faculty of the School of Engineering and Applied Science
University of Virginia
in partial fulfillment
of the requirements for the degree
Doctor of Philosophy
by

Andrew D. Rouillard

May

2013 
APPROVAL SHEET

The dissertation

is submitted in partial fulfillment of the requirements

for the degree of

Doctor of Philosophy

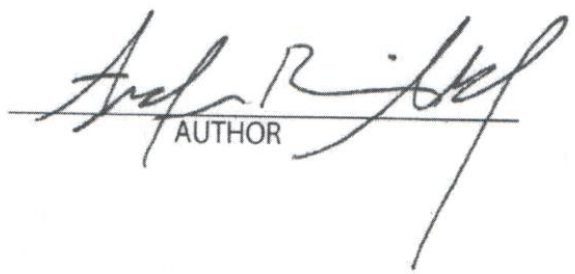

The dissertation has been read and approved by the examining committee:

\begin{tabular}{c} 
Dr. Jeffrey Holmes \\
\hline $\begin{array}{c}\text { Advisor } \\
\text { Dr. Brent French }\end{array}$ \\
\hline Dr. Douglas DeSimone \\
\hline Dr. Brian Helmke \\
\hline Dr. Jeffrey Saucerman \\
\hline
\end{tabular}

Accepted for the School of Engineering and Applied Science:

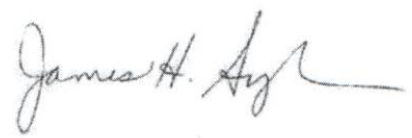

Dean, School of Engineering and Applied Science

May

2013 


\title{
Mechanical Regulation of Fibroblast Migration and COLLAGEN REMODELING IN HEALING MYOCARDIAL INFARCTS
}

\author{
Dissertation \\ presented to \\ The Faculty of the School of Engineering and Applied Science \\ University of Virginia \\ in partial fulfillment of the requirements for the degree of \\ Doctor of Philosophy in Biomedical Engineering
}

Andrew D. Rouillard

April 24, 2013 


\section{TABLE OF CONTENTS}

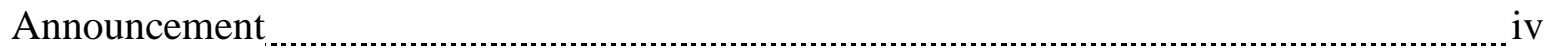

Abstract _ vii

Acknowledgments

Chapter 1: Introduction

1.1. Motivation

1.2. Background

1.3. Aims and Approach

1.4. Significance $\quad 10$

1.5. References

Chapter 2: $\quad$ Mechanical Regulation of Fibroblast Migration and

Collagen Remodeling in Healing Myocardial Infarcts

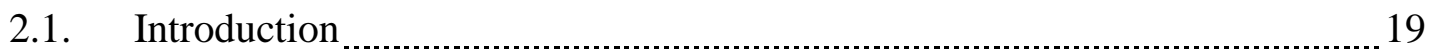

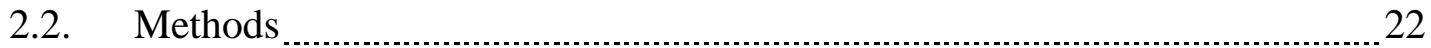

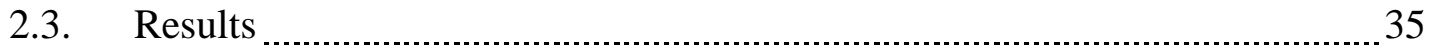

2.4. Discussion $\quad 39$

2.5. Conclusion

2.6. Figures and Tables

Appendix 2.A. Calculation of Guidance Cue Vectors _...............................66

Appendix 2.B. List of Model Parameters _................................................ 69

2.7. References 71

Chapter 3: Computational Screening of Candidate Processes of

Collagen Fiber Remodeling in Healing Myocardial Infarcts

3.1. Introduction $\quad 76$

3.2. Methods $\quad 7 \quad \ldots \ldots \ldots$

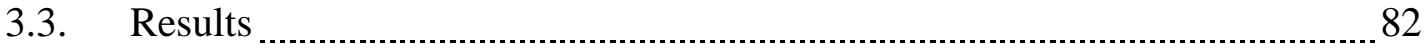

3.4. Discussion

3.5. Conclusion 101

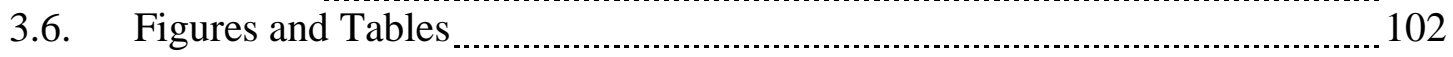

Appendix 3.A. Summary of selective degradation studies _............................ 114

Appendix 3.B. Calculation of selective degradation rate function _................ 117

3.7. References $\quad 120$ 


\section{TABLE OF CONTENTS}

Chapter 4: Empirical Approach to Predicting Cell Alignment and Biased Migration Driven by Mechanical, Structural, and Simultaneous Guidance Cues

4.1. Introduction 123

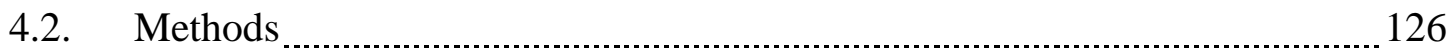

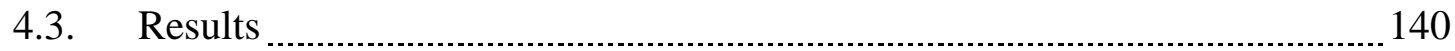

4.4. Discussion 146

4.5. Conclusion $\ldots$

4.6. Figures and Tables

4.7. References

Chapter 5: $\quad$ Mechanical Regulation of Fibroblast Migration in a Collagen-Fibrin Wound Model

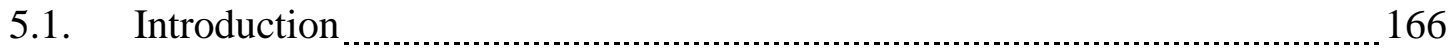

5.2. Methods 167

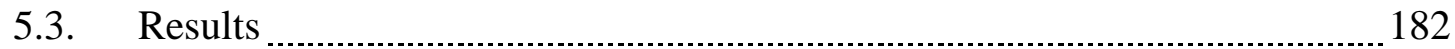

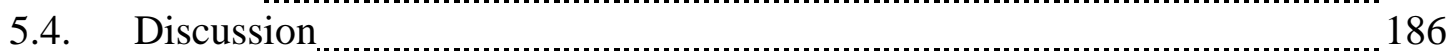

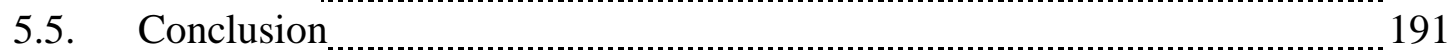

5.6. Figures and Tables

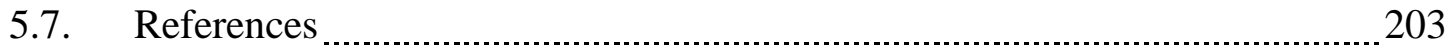

Chapter 6: Agent-Based Model with Integrated Finite Element Analysis for Investigation of the Mechanobiology of Healing Myocardial Infarcts

6.1. Introduction 206

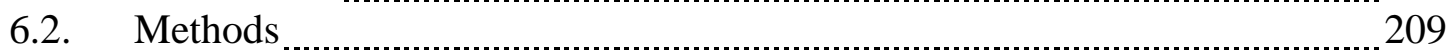

6.3. Results and Discussion $\ldots \ldots \ldots \ldots \ldots \ldots$

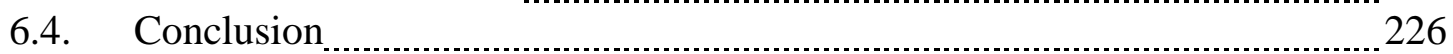

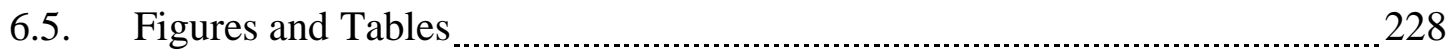

6.6. References

Chapter 7: $\quad$ Summary and Future Directions

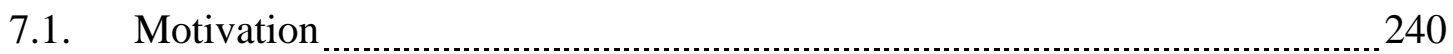

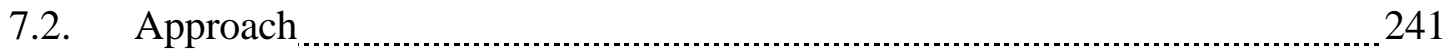

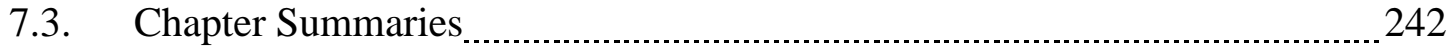

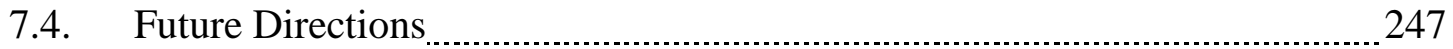

7.5. References 250 


\section{ANNOUNCEMENT}

Attention BME Faculty and Students

Andrew Rouillard will hold his Ph.D. defense:

Wednesday, April 24th at 9:00am in MR6 Room G501

Committee Members:

Dr. Brent French, BME, Committee Chair

Dr. Jeffrey Holmes, BME, Advisor

Dr. Douglas DeSimone, Cell Biology

Dr. Brian Helmke， BME

Dr. Jeffrey Saucerman, BME

Title:

Mechanical Regulation of Fibroblast Migration and Collagen Remodeling in Healing Myocardial Infarcts

\section{Brief Abstract:}

Each year, approximately 900,000 Americans experience a myocardial infarction. Most patients survive the initial event, making management of post-infarction healing and remodeling a high priority. After infarction, damaged myocardium is replaced by passive scar tissue, which impairs cardiac function and contributes toward adverse remodeling that leads to heart failure. Our lab has shown that the level of anisotropy of infarct scar is an important determinant of heart function. However, factors regulating infarct anisotropy are not well understood. Mechanical, 
structural, and chemical environmental cues have all been shown to regulate alignment of fibroblasts and collagen in vitro. A better understanding of how fibroblasts integrate these cues as they deposit and remodel extracellular matrix in a healing infarct is needed in order to develop interventions that modify infarct scar anisotropy for therapeutic benefit.

Our overall hypothesis was that collagen alignment in infarct scar tissue is primarily determined by mechanical cues that guide alignment of fibroblasts, which actively re-orient nearby collagen fibers. Our approach was to build a computational model of infarct healing and use it to test hypotheses about how the collagen fiber structure of infarct scar arises from the collective activity of individual fibroblasts sensing and responding to signals in their local environment.

We developed an agent-based model that accounted for the influence of mechanical, structural, and chemical cues on fibroblast alignment, collagen deposition, and collagen remodeling. We found that a mechanical cue was critical to reproducing the collagen alignment observed experimentally in healing infarcts. We also found that although active re-orientation of collagen fibers by fibroblasts could explain the collagen alignment observed in healing infarcts, aligned deposition of new collagen fibers by fibroblasts could also explain the experimental data. We performed parameter sensitivity analyses on the two alternative models, identified novel behaviors that each model predicted, and designed experiments that will further test the predictive capabilities of the models. In order to obtain experimental evidence in support of our modeling results, we created fibrin wounds in fibroblast-populated collagen gels, applied different mechanical restraints to the gels, and quantified the migration behavior of fibroblasts that entered the wounds. We found a strong guidance effect of anisotropic strain, indicating that 
mechanical cues can have strong effects on fibroblast behavior in wound-like environments. Finally, we developed a finite element model of an infarct and surrounding myocardium, and coupled the finite element model to our agent-based model, enabling the mechanical environment of the infarct to be updated in response to the structural remodeling that occurs during healing. The coupled model will be useful for further investigation of the mechanobiology of healing infarcts. Future versions could link the agent-based model to a finite element model of a whole heart, allowing the functional impact of different scar structures to be assessed in silico.

This work will aid the development of treatments that improve healing after myocardial infarction. We identified environmental factors and fibroblast behaviors that regulate anisotropy of infarct scar tissue and embedded this understanding in a computational framework that can be used to identify interventions that modify the properties of infarct scar for therapeutic benefit. 


\begin{abstract}
Each year, approximately 600,000 Americans experience a new myocardial infarction and 300,000 Americans experience a recurrent infarction. Most patients survive the initial event, making management of post-infarction healing and remodeling a high priority. After infarction, damaged myocardium is replaced by passive scar tissue, which impairs cardiac function and contributes toward adverse remodeling that leads to heart failure. The structural properties of infarct scar tissue partly determine how severely the infarct compromises cardiac function.
\end{abstract}

Our lab has shown that the level of anisotropy of infarct scar is an important determinant of heart function. However, factors regulating infarct anisotropy are not well understood. Mechanical, structural, and chemical environmental cues have all been shown to regulate alignment of fibroblasts and collagen in vitro. A better understanding of how fibroblasts integrate these cues as they deposit and remodel extracellular matrix in a healing infarct is needed in order to develop interventions that modify infarct scar anisotropy for therapeutic benefit.

The overall goal of this work was to understand the regulation of collagen fiber alignment during healing after myocardial infarction. Our overall hypothesis was that collagen alignment in infarct scar tissue 1) is primarily determined by mechanical cues (uniaxial strain) that guide fibroblast alignment and 2) requires active re-orientation of collagen fibers by fibroblasts. Our approach was to build a computational model of infarct healing and use it to test hypotheses about how the collagen fiber structure of infarct scar arises from the collective activity of individual fibroblasts sensing and responding to signals in their local environment. 
First, we tested the hypothesis that a mechanical guidance cue is necessary to explain collagen fiber alignment observed in infarct scars. We developed an agent-based model that accounted for the influence of mechanical, structural, and chemical cues on fibroblast alignment, collagen deposition, and collagen remodeling. We independently perturbed the mechanical, structural, and chemical cues in the model in order to determine how strongly each cue influenced the predicted collagen structure, and we found that a mechanical cue was critical to reproducing the collagen alignment observed experimentally in healing infarcts.

Next, we tested the hypothesis that active re-orientation of nearby collagen fibers by fibroblasts is necessary to explain collagen fiber alignment observed in infarct scars. We found that it was not necessary to include active re-orientation of collagen fibers by fibroblasts in our agent-based model in order to reproduce the collagen alignment and cell alignment observed in healing infarcts. The experimental data could also be explained by aligned deposition of collagen fibers along the axis of fibroblast alignment. We performed parameter sensitivity analyses on the aligned deposition and rotation models, identified novel behaviors that each model predicted, and designed experiments that will further test the predictive capabilities of the models.

Next, we developed an empirical approach to predicting fibroblast alignment and biased migration in response to environmental guidance cues. We formulated equations and fit for parameter values that could reproduce experimental measurements - reported in the literatureof cell alignment in response to mechanical (cyclic uniaxial stretch), structural (micro-ridged substrates), or simultaneous mechanical and structural guidance cues. We found empirical support for an interaction of structural and mechanical guidance cues during infarct healing that 
we anticipated with our agent-based model. Our mathematical framework made transparent areas where we have little understanding of how guidance cues interact, which motivates future experimentation.

Next, we tested the hypothesis that anisotropic mechanical boundary conditions increase the directionality and speed of migration of fibroblasts into a model wound. We created elliptical fibrin wounds in fibroblast-populated collagen gels and applied uniaxial mechanical restraint along the short or long axis of the fibrin wounds. We quantified fibroblast migration and found a strong guidance effect of short axis restraint relative to long axis restraint, without an increase in speed of fibroblast migration along the short axis of the wounds. This supported the finding of our computational study that mechanical cues can have strong effects on fibroblast behavior in wound-like environments.

Finally, we developed a finite element model of an infarct and surrounding myocardium and coupled the finite element model to our agent-based model of infarct healing. We defined coefficients of the constitutive equation of the finite element model as functions of the collagen area fraction and collagen mean vector length predicted by the agent-based model, and computed mechanical guidance cues in the agent-based model from strains predicted by the finite element model. The coupled model will be useful for investigating the mechanobiology of healing infarcts and future versions could link the agent-based model to a finite element model of a whole heart, allowing the functional impact of different scar structures to be assessed in silico. 
This work will aid the development of treatments that improve healing after myocardial infarction. We identified environmental factors and fibroblast behaviors that regulate anisotropy of infarct scar tissue and embedded this understanding in a computational framework that can be used to identify interventions that modify the properties of infarct scar for therapeutic benefit. 


\section{ACKNOWLEDGMENTS}

I thank Dr. Jeffrey Holmes for his dedicated mentorship, exceptional standards, and seemingly infinite patience. His enthusiastic yet exacting approach to science has been an inspiration.

I thank the members of my dissertation committee, Dr. Douglas DeSimone, Dr. Brent French, Dr. Brian Helmke, and Dr. Jeffrey Saucerman, for their invaluable advice, support, and interest with respect to my research endeavors.

I thank current and former members of the Cardiac Biomechanics Group, Elizabeth Ames, Jennifer Bartell, Tracy Burcin, Samantha Clarke, Dr. Gregory Fomovsky, Dr. Charles Haggart, Christian Moyer, Katherine Parker, and Dr. William Richardson, for their friendship, encouragement, sharing of ideas, and awesome sense of humor.

I thank my friends and family for their unwavering support of, confidence in, and excitement for my scientific pursuits. I thank my brother for making science and engineering look cool. I especially thank my parents, whose care, encouragement, and sacrifices provided me the opportunity and determination to pursue my goals.

Finally, I thank my wife, Celine, who has been my greatest fan and who inspires me to give my absolute best every day. She has helped me every step of the way, and I could not have completed this work without her by my side. 


\section{CHAPTER 1: Introduction}

\subsection{MOTIVATION}

Each year, approximately 600,000 Americans experience a new myocardial infarction and 300,000 Americans experience a recurrent infarction (Lloyd-Jones et al., 2010). Most patients survive the initial event, making management of post-infarction healing and remodeling a high priority (Lloyd-Jones et al., 2010). Current revascularization procedures and pharmacologic therapies seek to limit infarct extension, infarct expansion, and adverse remodeling of remote myocardium (Sutton \& Sharpe, 2000). Additionally, researchers are investigating regenerative therapies that may one day be able to restore contractile function to the infarct region (Forrester et al., 2003). These treatments are all motivated, at least in part, by the understanding that mechanical dysfunction is a key driver of the progression toward heart failure.

Treatments intended to reduce mechanical dysfunction of the heart or regenerate myocardium may be improved by controlling the anisotropy of infarct tissue. Anisotropy is an important feature of normal myocardium (Costa et al., 2001), which led our lab to study its importance in healing infarcts. Our lab has recently shown that the level of infarct anisotropy is a critical determinant of heart function following a large anterior infarction (Fomovsky et al., 2011, 2012a). However, factors regulating infarct anisotropy are not well understood. Mechanical, structural, and chemical environmental cues have all been shown to regulate alignment of fibroblasts and collagen in vitro (Dickinson et al., 1994; Lee et al., 2008; Melvin et al., 2011), but understanding of fibroblast behavior in the complex environment of a healing infarct is 
lacking. A better understanding of how fibroblasts integrate these cues as they deposit and remodel extracellular matrix in a healing infarct is needed in order to develop interventions that modify infarct scar anisotropy for therapeutic benefit.

\subsection{BACKGROUND}

\section{After myocardial infarction, the mechanical properties of the healing scar tissue determine}

the pathological outcome of the trauma. Over time, necrotic muscle is degraded and replaced with a collagen-rich scar tissue (Sun \& Weber, 2000; Lu et al., 2004; Holmes et al., 2005; Frangogiannis, 2008). Early in the healing process, if synthesis of extracellular matrix cannot keep pace with degradation of necrotic muscle, the infarct will lose mechanical strength and become susceptible to expansion and rupture (Bogen et al., 1980; Radhakrishnan et al., 1980; Holmes et al., 2005; Gao et al., 2005; Fang et al., 2007). Infarct expansion increases cardiac wall stresses globally due to the dilation of the heart (Bogaert et al., 2000; Holmes et al., 2005). Impairment of cardiac function depends not only on chamber dilation, but on the local mechanics of the infarct. Soft infarcts stretch during systole, which reduces ventricular ejection (Parmley et al., 1973; Laird \& Vellekoop, 1977; Bogen et al., 1980). Stiff infarcts resist stretching during diastole, which increases the wall stress needed to fill the ventricle (Janz \& Waldron, 1978; Bogen et al., 1980). A stiff infarct will also interfere with shortening and thickening of surrounding myocardium (Holmes et al., 1997). Over the long term, the infarct imposes a persistent disturbance in the mechanics of the heart, which triggers adverse remodeling of healthy myocardium (fibrosis and hypertrophy) in reaction to higher stresses and loss of contractile myocardium (Pfeffer \& Braunwald, 1990; Jugdutt, 2003). 


\section{Anisotropy of infarct scar tissue can reduce left ventricular dysfunction and may limit} adverse remodeling. Studies have shown that infarct scar tissue is not simply soft or stiff, but anisotropic, with different mechanical properties in different directions (Gupta et al., 1994; Holmes et al., 1997; Costa et al., 2001). Using Continuity, a finite-element model of the dog heart, our lab simulated an anteroapical infarct and found that anisotropic scar, with greater stiffness in the longitudinal direction, preserved ventricular function better than isotropic scar (Fomovsky et al., 2011). This result was confirmed in a dog study that used a patch to longitudinally reinforce an acute infarct (Fomovsky et al., 2012a). By reducing the mechanical disturbance imposed by the scar, anisotropy may limit adverse ventricular remodeling.

\section{Fibroblasts mediate the development of the mechanical properties of infarct tissue by} regulating the composition and structure of the extracellular matrix. Fibroblasts are the cell type primarily responsible for depositing and organizing collagen fibers in scar tissue (Kanekar et al., 1998; Brown et al., 2005; Camelliti et al., 2005; Baudino et al., 2006; Porter \& Turner, 2009; Souders et al., 2009). Fibroblasts assemble collagen fibers, secrete proteolytic and crosslinking enzymes, and apply traction forces that physically deform collagen fiber networks (Harris et al., 1981; Carver et al., 1991; Canty et al., 2004; Thomopoulos et al., 2005; Kadler et al., 2008). Fibroblasts create the microstructure, that is, the density, orientation, thickness, and crosslinking of collagen fiber bundles, that explains the mechanical properties of the infarct (Sellaro et al., 2006; Wagenseil \& Okamoto, 2007; Kroon, 2010). In particular, anisotropy is determined by the orientation distribution of the collagen fibers (Sacks, 2003; Thomopoulos et al., 2005; Lee et al., 2008). As the microstructure of the infarct changes, the mechanical 
properties of the infarct change, and in turn, the mechanical loads and deformations, both in the infarct and in the rest of the heart wall, change.

\section{In vivo evidence has identified mechanical and structural guidance cues as potential} determinants of infarct collagen alignment. A wide range of studies have reported differing structural and mechanical properties of infarcts (Whittaker et al., 1989; Gupta et al., 1994; Omens et al., 1997; Holmes et al., 1997; Fomovsky \& Holmes, 2010). It is difficult to make inferences about the determinants of infarct scar microstructure due to the many variables that change from study to study, such as animal model, infarct location, infarct size, and infarct shape. Furthermore, other variables can change as any one of these variables changes, such as muscle fiber orientation, deformation pattern through the cardiac cycle, and chemokine concentration profile. Our lab performed a cryoinfarction study that independently varied infarct location and shape in a consistent animal model (rat) and found that infarct scar collagen alignment depended on location but not shape (Fomovsky et al., 2012b). Scar collagen fiber alignment was correlated with both the direction of greatest systolic deformation and the direction of the myofibers that the scar replaced. This study suggests that mechanical deformations and/or structural templates regulate collagen alignment in infarct scar.

In vitro evidence has identified mechanical, structural, and chemical cues that guide fibroblast alignment as potential determinants of infarct collagen alignment. Fibroblasts in collagen rich tissues, such as scar and tendon, are typically found aligned with the direction of local collagen fibers (Canty et al., 2004, 2006; Richardson et al., 2007; Kapacee et al., 2008). This suggests that fibroblasts organize collagen in an oriented manner (Den Braber et al., 1998; 
Wang et al., 2003). According to this model, any cue for fibroblast alignment may subsequently cause collagen alignment. In vitro studies have shown that fibroblasts will align in the direction of uniaxial mechanical stretch (Eastwood et al., 1998; Wang \& Grood, 2000; Neidlinger-Wilke et al., 2001; Raeber et al., 2008; Lee et al., 2008; Hsu et al., 2009; Steward et al., 2010; Pang et $a l ., 2011$ ), in the direction of aligned matrix fibers or micropatterned ridges (Guido \& Tranquillo, 1993; Dickinson et al., 1994; Sutherland et al., 2005; Loesberg et al., 2007; Doyle et al., 2009; Sun et al., 2010), and in the direction of chemokine gradients (Knapp et al., 1999; Grinnell et al., 2006; Melvin et al., 2011). Mechanical, structural, and chemical cues are all present during infarct healing, which makes all of them potential determinants of infarct collagen alignment.

\section{The dynamics of wound infiltration by fibroblasts may be regulated by the mechanical} boundary conditions acting on the wound. Cues that orient fibroblasts tend to direct fibroblast migration as well. Chemotaxis is a well-documented phenomenon thought to be responsible for guiding infiltration of fibroblasts and other cell types into wounds (Mutsaers et al., 1997; Haugh, 2006; McDougall et al., 2006; Shaw \& Martin, 2009). It is possible that mechanical guidance also regulates wound infiltration. As mentioned above, in an anisotropic mechanical environment, fibroblasts have been shown to align in the direction of greatest stiffness or stretch. This effect may cause fibroblasts infiltrating a wound to follow trajectories along the direction of greatest stiffness (Raeber et al., 2008; Pang et al., 2011). Also, the speed of fibroblast migration has been shown to depend on substrate stiffness (Lo et al., 2000; Discher et al., 2005; Dokukina \& Gracheva, 2010). This effect may cause fibroblasts to infiltrate a wound faster along the direction of greatest stiffness. The mechanical boundary conditions acting on a wound may influence both the pattern and rate of infiltration of fibroblasts. 


\section{Mechanically-induced collagen alignment may be regulated at the level of collagen} deposition, collagen reorientation, or collagen degradation. Tendon research has suggested that collagen fibrils are secreted from fibripositors oriented along cell protrusions, which causes co-alignment of deposited collagen with the axis of cell elongation (Birk \& Trelstad, 1986; den Braber et al., 1998; Wang et al., 2003; Canty et al., 2004). In vitro studies of fibroblasts in collagen gels have shown that spindle-shaped fibroblasts exert traction forces predominantly along their axis of elongation, and these traction forces cause nearby collagen fibers to rotate into alignment with the cell (Petroll et al., 2003, 2008; Rape et al., 2011). Since anisotropic mechanical boundary conditions have been shown to cause alignment of fibroblasts, either of these two mechanisms could explain mechanically-induced collagen alignment. Additionally, there are two plausible mechanisms that do not depend on fibroblast alignment. The kinetics of enzyme-mediated degradation of collagen fibers have been shown to depend on the tensile load or stretch applied to the fiber (Wyatt et al., 2009; Bhole et al., 2009; Zareian et al., 2010; Araújo et al., 2011). Also, in the presence of anisotropic mechanical boundary conditions, anisotropic deformation will occur regardless of whether fibroblast traction forces are coordinated in a particular direction or randomly oriented (Fernandez \& Bausch, 2009). Anisotropic deformation of a collagen matrix will cause rotation of collagen fibers toward the direction of greatest stiffness (Roeder et al., 2004; Matsumoto et al., 2007; Vader et al., 2009; Fernandez \& Bausch, 2009). Any one or some combination of these mechanisms may dominate during infarct healing. 


\subsection{AIMS AND APPROACH}

The overall goal of this work was to understand the regulation of collagen fiber alignment during healing after myocardial infarction. Our overall hypothesis was that collagen alignment in infarct scar tissue 1) is primarily determined by mechanical cues (uniaxial strain) that guide fibroblast alignment (Katsumi et al., 2002; Fomovsky et al., 2012b) and 2) requires active reorientation of collagen fibers by fibroblasts (Petroll et al., 2003; Lee et al., 2008; Pang et al., 2011). Alternatively, structural (pre-existing aligned fibers) (Dickinson et al., 1994; Sun et al., 2010) and chemical (chemokine gradients) (Knapp et al., 1999; Melvin et al., 2011) guidance

cues may influence the alignment of fibroblasts in healing infarcts. Also, aligned deposition of collagen fibers by fibroblasts (Wang et al., 2003; Richardson et al., 2007; Kapacee et al., 2008) and selective degradation of fibers experiencing low strain (Huang \& Yannas, 1977; Flynn et al., 2010) may contribute toward alignment of collagen fibers in healing infarcts.

Our approach was to build a computational model of infarct healing and use it to test hypotheses about how collagen fiber alignment is regulated during infarct healing. Our hypotheses revolved around the question of how the collagen fiber structure of infarct scar arises from the collective activity of individual fibroblasts sensing and responding to signals in their local environment. We decided to build an agent-based model, because such models are well-suited to studying how behaviors of individual agents (e.g. fibroblasts) within a system (e.g. infarct) give rise to emergent properties of the whole system (Chavali et al., 2008). 
In Chapter 2, we present our agent-based model of infarct healing, which incorporated experimentally measured fibroblast behaviors, environmental conditions, and parameter values in order to predict the structural properties of the collagen scar formed after myocardial infarction. We independently perturbed the mechanical, structural, and chemical cues in the model in order to determine how strongly each cue influence the predicted collagen structure, and we tested the specific hypothesis that a mechanical guidance cue is necessary to explain collagen fiber alignment observed in infarct scars.

In Chapter 3, we used the agent-based model to investigate the effects that three candidate processes of collagen fiber remodeling-aligned deposition, fiber rotation, and selective degradation — could have on collagen fiber alignment in healing infarcts. We measured the strength of cell alignment in infarct scars for use as an additional model constraint. We then modified our agent-based model to allow collagen fiber remodeling via aligned deposition only, rotation only, or selective degradation only and tested the specific hypothesis that active reorientation of nearby collagen fibers (fiber rotation) by fibroblasts is necessary to explain collagen fiber alignment observed in infarct scars.

In Chapter 4, we assembled and analyzed literature data in order to develop an empirical approach to predicting fibroblast alignment and migration in response to environmental guidance cues. One of our findings in Chapter 2 was that although a mechanical guidance cue was critical to predicting collagen fiber alignment in infarct scar, structural guidance cues had important effects. This motivated us to assemble data from studies investigating how fibroblast alignment and migration are influenced by mechanical, structural, and simultaneous mechanical and 
structural guidance cues. We used the literature data to formulate an approach to predicting fibroblast alignment and migration from measureable characteristics of mechanical and structural guidance cues, such as strain amplitudes, fiber size, and fiber spacing.

In Chapter 5, we developed a wound healing model using a three-dimensional collagen-fibrin gel composite that would allow us to address questions about the interaction of guidance cues during wound healing in a controlled, readily observable setting. We sought to advance our finding of the important influence of mechanical environment on fibroblast alignment during infarct healing by asking if mechanical environment regulated fibroblast migration in our experimental wound model. We created elliptical fibrin "wounds" in fibroblast-populated collagen gels and applied uniaxial mechanical restraint at the edges of the collagen gels along the short or long axis of the fibrin wounds. We tested the specific hypothesis that anisotropic mechanical boundary conditions increase the directionality and speed of migration of fibroblasts into a model wound.

In Chapter 6, we present an approach to coupling a finite element model to our agent-based model of infarct healing. Ultimately, in order to predict how interventions that change the collagen fiber structure of infarct scar affect heart function, we must first compute the mechanical properties of the scar, and then use a finite element model of the heart to compute how the scar affects heart function. This task is complicated by the fact that infarct strains, and therefore mechanical guidance cues, are partly determined by the structural properties of the infarct. A feedback loop may exist, where the pattern of mechanical strain guides structural remodeling of the infarct, which changes the infarct strains. We developed a finite element 
model of an infarct and an approach to coupling the finite element model to our agent-based model of infarct healing. We used the finite element model to test how sensitive infarct strains are to variations of infarct structure.

\subsection{SIGNIFICANCE}

The significance of this work is that it will aid the development of treatments that improve healing after myocardial infarction. We identified environmental factors and fibroblast behaviors that regulate anisotropy of infarct scar tissue and embedded this understanding in a computational framework that can be used to identify interventions that modify the properties of infarct scar for therapeutic benefit. Because a myriad of potential therapies for myocardial infarction are currently in development (Johnston et al., 2009; Chung et al., 2010; Morita et al., 2011; Rane \& Christman, 2011), we can use the model to anticipate unintended impacts of such interventions on the collagen fiber structure of infarct scar, and ultimately on heart function. This research has the potential to reduce the occurrence of heart failure and improve the quality of life of patients after myocardial infarction. 


\subsection{REFERENCES}

Araújo AD, Majumdar A, Parameswaran H, Yi E, Spencer JL, Nugent MA \& Suki B (2011). Dynamics of enzymatic digestion of elastic fibers and networks under tension. Proceedings of the National Academy of Sciences 108, 9414-9419.

Baudino TA, Carver W, Giles W \& Borg TK (2006). Cardiac fibroblasts: friend or foe? American Journal of Physiology Heart and Circulatory Physiology 291, H1015-H1026.

Bhole AP, Flynn BP, Liles M, Saeidi N, Dimarzio CA \& Ruberti JW (2009). Mechanical strain enhances survivability of collagen micronetworks in the presence of collagenase: implications for load-bearing matrix growth and stability. Philosophical Transactions of the Royal Society A: Mathematical, Physical and Engineering Sciences 367, 3339-3362.

Birk DE \& Trelstad RL (1986). Extracellular compartments in tendon morphogenesis: collagen fibril, bundle, and macroaggregate formation. The Journal of Cell Biology 103, 231-240.

Bogaert J, Bosmans H, Maes A, Suetens P, Marchal G \& Rademakers FE (2000). Remote myocardial dysfunction after acute anterior myocardial infarction: impact of left ventricular shape on regional function. Journal of the American College of Cardiology 35, 1525-1534.

Bogen DK, Rabinowitz SA, Needleman A, McMahon TA \& Abelmann WH (1980). An analysis of the mechanical disadvantage of myocardial infarction in the canine left ventricle. Circulation Research 47, 728-741.

Den Braber ET, De Ruijter JE, Ginsel LA, Von Recum AF \& Jansen JA (1998). Orientation of ECM protein deposition, fibroblast cytoskeleton, and attachment complex components on silicone microgrooved surfaces. Journal of Biomedical Materials Research 40, 291-300.

Brown RD, Ambler SK, Mitchell MD \& Long CS (2005). The cardiac fibroblast: therapeutic target in myocardial remodeling and failure. Annual Review of Pharmacology and Toxicology 45, 657-687.

Camelliti P, McCulloch AD \& Kohl P (2005). Microstructured cocultures of cardiac myocytes and fibroblasts: a two-dimensional in vitro model of cardiac tissue. Microscopy and Microanalysis 11, 249-259.

Canty EG, Lu Y, Meadows RS, Shaw MK, Holmes DF \& Kadler KE (2004). Coalignment of plasma membrane channels and protrusions (fibripositors) specifies the parallelism of tendon. The Journal of Cell Biology 165, 553-563.

Canty EG, Starborg T, Lu Y, Humphries SM, Holmes DF, Meadows RS, Huffman A, O'Toole ET \& Kadler KE (2006). Actin filaments are required for fibripositor-mediated collagen fibril alignment in tendon. The Journal of Biological Chemistry 281, 38592-38598. 
Carver W, Nagpal ML, Nachtigal M, Borg TK \& Terracio L (1991). Collagen expression in mechanically stimulated cardiac fibroblasts. Circulation Research 69, 116-122.

Chavali AK, Gianchandani EP, Tung KS, Lawrence MB, Peirce SM \& Papin JA (2008). Characterizing emergent properties of immunological systems with multi-cellular rulebased computational modeling. Trends in Immunology 29, 589-599.

Chung ES, Dan D, Solomon SD, Bank AJ, Pastore J, Iyer A, Berger RD, Franklin JO, Jones G, Machado C \& Stolen CM (2010). Effect of peri-infarct pacing early after myocardial infarction: results of the prevention of myocardial enlargement and dilatation post myocardial infarction study. Circulation Heart Failure 3, 650-658.

Costa KD, Holmes JW \& McCulloch AD (2001). Modelling cardiac mechanical properties in three dimensions. Philosophical Transactions of the Royal Society A: Mathematical, Physical and Engineering Sciences 359, 1233-1250.

Dickinson RB, Guido S \& Tranquillo RT (1994). Biased cell migration of fibroblasts exhibiting contact guidance in oriented collagen gels. Annals of Biomedical Engineering 22, 342-356.

Discher DE, Janmey P \& Wang Y-L (2005). Tissue cells feel and respond to the stiffness of their substrate. Science 310, 1139-1143.

Dokukina I V \& Gracheva ME (2010). A model of fibroblast motility on substrates with different rigidities. Biophysical Journal 98, 2794-2803.

Doyle AD, Wang FW, Matsumoto K \& Yamada KM (2009). One-dimensional topography underlies three-dimensional fibrillar cell migration. The Journal of Cell Biology 184, 481490.

Eastwood M, Mudera VC, McGrouther DA \& Brown RA (1998). Effect of precise mechanical loading on fibroblast populated collagen lattices: morphological changes. Cell Motility and the Cytoskeleton 40, 13-21.

Fang L, Gao X-M, Moore X-L, Kiriazis H, Su Y, Ming Z, Lim YL, Dart AM \& Du X-J (2007). Differences in inflammation, MMP activation and collagen damage account for gender difference in murine cardiac rupture following myocardial infarction. Journal of Molecular and Cellular Cardiology 43, 535-544.

Fernandez P \& Bausch AR (2009). The compaction of gels by cells: a case of collective mechanical activity. Integrative Biology 1, 252-259.

Flynn BP, Bhole AP, Saeidi N, Liles M, DiMarzio CA \& Ruberti JW (2010). Mechanical strain stabilizes reconstituted collagen fibrils against enzymatic degradation by mammalian collagenase matrix metalloproteinase 8 (MMP-8). PLoS ONE 5, e12337. 
Fomovsky GM, Clark SA, Parker KM, Ailawadi G \& Holmes JW (2012a). Anisotropic reinforcement of acute anteroapical infarcts improves pump function. Circulation Heart Failure 5, 515-522.

Fomovsky GM \& Holmes JW (2010). Evolution of scar structure, mechanics, and ventricular function after myocardial infarction in the rat. American Journal of Physiology: Heart and Circulatory Physiology 298, H221-H228.

Fomovsky GM, Macadangdang JR, Ailawadi G \& Holmes JW (2011). Model-based design of mechanical therapies for myocardial infarction. Journal of Cardiovascular Translational Research 4, 82-91.

Fomovsky GM, Rouillard AD \& Holmes JW (2012b). Regional mechanics determine collagen fiber structure in healing myocardial infarcts. Journal of Molecular and Cellular Cardiology 52, 1083-1090.

Forrester JS, Price MJ \& Makkar RR (2003). Stem cell repair of infarcted myocardium: an overview for clinicians. Circulation 108, 1139-1145.

Frangogiannis NG (2008). The immune system and cardiac repair. Pharmacological Research 58, 88-111.

Gao X-M, Xu Q, Kiriazis H, Dart AM \& Du X-J (2005). Mouse model of post-infarct ventricular rupture: time course, strain- and gender-dependency, tensile strength, and histopathology. Cardiovascular Research 65, 469-477.

Grinnell F, Rocha LB, Iucu C, Rhee S \& Jiang H (2006). Nested collagen matrices: a new model to study migration of human fibroblast populations in three dimensions. Experimental Cell Research 312, 86-94.

Guido S \& Tranquillo RT (1993). A methodology for the systematic and quantitative study of cell contact guidance in oriented collagen gels. Journal of Cell Science 105, 317-331.

Gupta KB, Ratcliffe MB, Fallert MA, Edmunds LH \& Bogen DK (1994). Changes in passive mechanical stiffness of myocardial tissue with aneurysm formation. Circulation 89, 23152326.

Harris AK, Stopak D \& Wild P (1981). Fibroblast traction as a mechanism for collagen morphogenesis. Nature 290, 249-251.

Haugh JM (2006). Deterministic model of dermal wound invasion incorporating receptormediated signal transduction and spatial gradient sensing. Biophysical Journal 90, 22972308.

Holmes JW, Borg TK \& Covell JW (2005). Structure and mechanics of healing myocardial infarcts. Annual Review of Biomedical Engineering 7, 223-253. 
Holmes JW, Nuñez JA \& Covell JW (1997). Functional implications of myocardial scar structure. American Journal of Physiology: Heart and Circulatory Physiology 272, H2123$\mathrm{H} 2130$.

Hsu H-J, Lee C-F \& Kaunas R (2009). A dynamic stochastic model of frequency-dependent stress fiber alignment induced by cyclic stretch. PLOS ONE 4, e4853.

Huang C \& Yannas I (1977). Mechanochemical studies of enzymatic degradation of insoluble collagen fibers. Journal of Biomedical Materials Research 8, 137-154.

Janz RF \& Waldron RJ (1978). Predicted effect of chronic apical aneurysms on the passive stiffness of the human left ventricle. Circulation Research 42, 255-263.

Johnston P V, Sasano T, Mills K, Evers R, Lee S-T, Smith RR, Lardo AC, Lai S, Steenbergen C, Gerstenblith G, Lange R \& Marbán E (2009). Engraftment, differentiation, and functional benefits of autologous cardiosphere-derived cells in porcine ischemic cardiomyopathy. Circulation 120, 1075-1083.

Jugdutt BI (2003). Ventricular remodeling after infarction and the extracellular collagen matrix: when is enough enough? Circulation 108, 1395-1403.

Kadler KE, Hill A \& Canty-Laird EG (2008). Collagen fibrillogenesis: fibronectin, integrins, and minor collagens as organizers and nucleators. Current Opinion in Cell Biology 20, 495-501.

Kanekar S, Hirozanne T, Terracio L \& Borg TK (1998). Cardiac fibroblasts: form and function. Cardiovascular Pathology 7, 127-133.

Kapacee Z, Richardson SH, Lu Y, Starborg T, Holmes DF, Baar K \& Kadler KE (2008). Tension is required for fibripositor formation. Matrix Biology 27, 371-375.

Katsumi A, Milanini J, Kiosses WB, Del Pozo MA, Kaunas R, Chien S, Hahn KM \& Schwartz MA (2002). Effects of cell tension on the small GTPase Rac. The Journal of Cell Biology 158, $153-164$.

Knapp DM, Helou EF \& Tranquillo RT (1999). A fibrin or collagen gel assay for tissue cell chemotaxis: assessment of fibroblast chemotaxis to GRGDSP. Experimental Cell Research 247, 543-553.

Kroon M (2010). Modeling of fibroblast-controlled strengthening and remodeling of uniaxially constrained collagen gels. Journal of Biomechanical Engineering 132, 111008.

Laird JD \& Vellekoop HP (1977). Time course of passive elasticity of myocardial tissue following experimental infarction in rabbits and its relation to mechanical dysfunction. Circulation Research 41, 715-721. 
Lee EJ, Holmes JW \& Costa KD (2008). Remodeling of engineered tissue anisotropy in response to altered loading conditions. Annals of Biomedical Engineering 36, 1322-1334.

Lloyd-Jones D et al. (2010). Heart disease and stroke statistics - 2010 update: a report from the American Heart Association. Circulation 121, e46-e215.

Lo C-M, Wang H-B, Dembo M \& Wang Y (2000). Cell movement is guided by the rigidity of the substrate. Biophysical Journal 79, 144-152.

Loesberg WA, Te Riet J, Van Delft FCMJM, Schön P, Figdor CG, Speller S, Van Loon JJWA, Walboomers XF \& Jansen JA (2007). The threshold at which substrate nanogroove dimensions may influence fibroblast alignment and adhesion. Biomaterials 28, 3944-3951.

Lu L, Zhang JQ, Ramires FJ \& Sun Y (2004). Molecular and cellular events at the site of myocardial infarction: from the perspective of rebuilding myocardial tissue. Biochemical and Biophysical Research Communications 320, 907-913.

Matsumoto T, Sasaki J, Alsberg E, Egusa H, Yatani H \& Sohmura T (2007). Three-dimensional cell and tissue patterning in a strained fibrin gel system. PLOS ONE 2, e1211.

McDougall S, Dallon J, Sherratt J \& Maini P (2006). Fibroblast migration and collagen deposition during dermal wound healing: mathematical modelling and clinical implications. Philosophical Transactions of the Royal Society A: Mathematical, Physical and Engineering Sciences 364, 1385-1405.

Melvin AT, Welf ES, Wang Y, Irvine DJ \& Haugh JM (2011). In chemotaxing fibroblasts, both high-fidelity and weakly biased cell movements track the localization of PI3K signaling. Biophysical Journal 100, 1893-1901.

Morita M, Eckert CE, Matsuzaki K, Noma M, Ryan LP, Burdick JA, Jackson BM, Gorman JH, Sacks MS \& Gorman RC (2011). Modification of infarct material properties limits adverse ventricular remodeling. Annals of Thoracic Surgery 92, 617-625.

Mutsaers SE, Bishop JE, McGrouther G \& Laurent GJ (1997). Mechanisms of tissue repair: from wound healing to fibrosis. The International Journal of Biochemistry and Cell Biology 29, $5-17$.

Neidlinger-Wilke C, Grood ES, Wang J H-C, Brand RA \& Claes L (2001). Cell alignment is induced by cyclic changes in cell length: studies of cells grown in cyclically stretched substrates. Journal of Orthopaedic Research 19, 286-293.

Omens JH, Miller TR \& Covell JW (1997). Relationship between passive tissue strain and collagen uncoiling during healing of infarcted myocardium. Cardiovascular Research 33, $351-358$. 
Pang Y, Wang X, Lee D \& Greisler HP (2011). Dynamic quantitative visualization of single cell alignment and migration and matrix remodeling in 3-D collagen hydrogels under mechanical force. Biomaterials 32, 3776-3783.

Parmley WW, Chuck L, Kivowitz C, Matloff JM \& Swan HJC (1973). In vitro length-tension relations of human ventricular aneurysms. The American Journal of Cardiology 32, 889894.

Petroll WM, Ma L \& Jester J V (2003). Direct correlation of collagen matrix deformation with focal adhesion dynamics in living corneal fibroblasts. Journal of Cell Science 116, 14811491.

Petroll WM, Ma L, Kim A, Ly L \& Vishwanath M (2008). Dynamic assessment of fibroblast mechanical activity during Rac-induced cell spreading in 3-D culture. Journal of Cellular Physiology 217, 162-171.

Pfeffer MA \& Braunwald E (1990). Ventricular remodeling after myocardial infarction: experimental observations and clinical implications. Circulation 81, 1161-1172.

Porter KE \& Turner NA (2009). Cardiac fibroblasts: at the heart of myocardial remodeling. Pharmacology \& Therapeutics 123, 255-278.

Radhakrishnan S, Ghista DN \& Jayaraman G (1980). Mechanical analysis of the development of left ventricular aneurysms. Journal of Biomechanics 13, 1031-1039.

Raeber GP, Lutolf MP \& Hubbell JA (2008). Part II: fibroblasts preferentially migrate in the direction of principal strain. Biomechanics and Modeling in Mechanobiology 7, 215-225.

Rane AA \& Christman KL (2011). Biomaterials for the treatment of myocardial infarction: a 5year update. Journal of the American College of Cardiology 58, 2615-2629.

Rape AD, Guo W-H \& Wang Y-L (2011). The regulation of traction force in relation to cell shape and focal adhesions. Biomaterials 32, 2043-2051.

Richardson SH, Starborg T, Lu Y, Humphries SM, Meadows RS \& Kadler KE (2007). Tendon development requires regulation of cell condensation and cell shape via cadherin-11mediated cell-cell junctions. Molecular and Cellular Biology 27, 6218-6228.

Roeder BA, Kokini K, Robinson JP \& Voytik-Harbin SL (2004). Local, three-dimensional strain measurements within largely deformed extracellular matrix constructs. Journal of Biomechanical Engineering 126, 699-708.

Sacks MS (2003). Incorporation of experimentally-derived fiber orientation into a structural constitutive model for planar collagenous tissues. Journal of Biomechanical Engineering 125, 280-287. 
Sellaro TL, Hildebrand D, Lu Q, Vyavahare N, Scott M \& Sacks MS (2006). Effects of collagen fiber orientation on the response of biologically derived soft tissue biomaterials to cyclic loading. Journal of Biomedical Materials Research Part A 80, 194-205.

Shaw TJ \& Martin P (2009). Wound repair at a glance. Journal of Cell Science 122, 3209-3213.

Souders CA, Bowers SLK \& Baudino TA (2009). Cardiac fibroblast: the renaissance cell. Circulation Research 105, 1164-1176.

Steward RL, Cheng C-M, Wang DL \& LeDuc PR (2010). Probing cell structure responses through a shear and stretching mechanical stimulation technique. Cell Biochemistry and Biophysics 56, 115-124.

Sun J, Ding Y, Lin NJ, Zhou J, Ro H, Soles CL, Cicerone MT \& Lin-Gibson S (2010). Exploring cellular contact guidance using gradient nanogratings. Biomacromolecules 11, 3067-3072.

Sun Y \& Weber KT (2000). Infarct scar: a dynamic tissue. Cardiovascular Research 46, 250256.

Sutherland J, Denyer M \& Britland S (2005). Contact guidance in human dermal fibroblasts is modulated by population pressure. Journal of Anatomy 206, 581-587.

Sutton MG \& Sharpe N (2000). Left ventricular remodeling after myocardial infarction: pathophysiology and therapy. Circulation 101, 2981-2988.

Thomopoulos S, Fomovsky GM \& Holmes JW (2005). The development of structural and mechanical anisotropy in fibroblast populated collagen gels. Journal of Biomechanical Engineering 127, 742-750.

Vader D, Kabla A, Weitz D \& Mahadevan L (2009). Strain-induced alignment in collagen gels. PLOS ONE 4, e5902.

Wagenseil JE \& Okamoto RJ (2007). Modeling cell and matrix anisotropy in fibroblast populated collagen vessels. Biomechanics and Modeling in Mechanobiology 6, 151-162.

Wang JH-C \& Grood ES (2000). The strain magnitude and contact guidance determine orientation response of fibroblasts to cyclic substrate strains. Connective Tissue Research 41, 29-36.

Wang JH-C, Jia F, Gilbert TW \& Woo SL-Y (2003). Cell orientation determines the alignment of cell-produced collagenous matrix. Journal of Biomechanics 36, 97-102.

Whittaker P, Boughner DR \& Kloner RA (1989). Analysis of healing after myocardial infarction using polarized light microscopy. The American Journal of Pathology 134, 879-893. 
Wyatt KE-K, Bourne JW \& Torzilli PA (2009). Deformation-dependent enzyme mechanokinetic cleavage of type I collagen. Journal of Biomechanical Engineering 131, 051004.

Zareian R, Church KP, Saeidi N, Flynn BP, Beale JW \& Ruberti JW (2010). Probing collagen/enzyme mechanochemistry in native tissue with dynamic, enzyme-induced creep. Langmuir 26, 9917-9926. 
CHAPTER 2: Mechanical Regulation of Fibroblast Migration and Collagen Remodeling in Healing Myocardial Infarcts

\subsection{INTRODUCTION}

The level of infarct anisotropy is a critical determinant of heart function following a large anterior infarction (Fomovsky et al., 2011, 2012a), but regulation of infarct anisotropy is not well understood. Mechanical, structural, and chemical environmental cues have all been shown to regulate alignment of fibroblasts and collagen in vitro (Dickinson et al., 1994; Lee et al., 2008; Melvin et al., 2011). An understanding of how fibroblasts integrate these cues as they deposit and remodel extracellular matrix in a healing infarct is needed in order to develop therapies that improve heart function by modifying infarct scar anisotropy.

Fibroblasts are the cell type primarily responsible for creating the microstructure, that is, the density, orientation, thickness, and crosslinking of collagen fiber bundles, that explains the mechanical properties of infarct scar tissue (Sacks, 2003; Souders et al., 2009). Fibroblasts assemble collagen fibers, secrete proteolytic and crosslinking enzymes, and apply traction forces that physically deform collagen fiber networks (Thomopoulos et al., 2005; Kadler et al., 2008). Fibroblasts can deposit collagen fibers oriented along their long axis and rotate nearby collagen fibers toward co-alignment with their long axis (Petroll et al., 2003; Canty et al., 2004). Consequently, any cue for fibroblast alignment may cause collagen alignment. In vitro studies have shown that fibroblasts align in response to uniaxial stretch (Neidlinger-Wilke et al., 2001; Pang et al., 2011), micropatterned ridges or aligned fibers (Dickinson et al., 1994; Wang et al., 
2003), and chemokine gradients (Knapp et al., 1999; Melvin et al., 2011). These environmental guidance cues have been shown to cause fibroblasts to deposit anisotropic extracellular matrices or remodel isotropic matrices into anisotropic matrices (Wang et al., 2003; Thomopoulos et al., 2005).

Several studies have reported differing anisotropic properties of infarcts (Whittaker et al., 1989; Gupta et al., 1994; Holmes et al., 1997; Fomovsky \& Holmes, 2010). It is difficult to make inferences about the determinants of infarct scar anisotropy due to the many variables that change from study to study, such as animal model, infarct location, infarct size, and infarct shape, but there is some evidence supporting a role for chemical, structural, and mechanical cues. In a computational model of skin wound healing, McDougall et al. (2006) showed that wound geometry can regulate collagen alignment by determining the spatial pattern of chemokine gradients. Zimmerman et al. (2000) studied the development of collagen alignment in healing pig infarcts and concluded that the initial matrix alignment in native myocardium determines the alignment of collagen in replacement scar. Recently, we performed a cryoinfarction study that independently varied the location and shape of infarcts in rats and found that infarct scar collagen alignment was correlated with the deformation pattern of the infarct, which depended on infarct location but not shape (Fomovsky et al., 2012b). At the equator of the left ventricle, infarcts stretched primarily in the circumferential direction and developed scar tissue with circumferentially aligned collagen fibers. At the apex of the heart, infarcts stretched in the circumferential and longitudinal directions and developed scar tissue with a random collagen fiber orientation. This suggested that mechanical deformations regulate collagen alignment in infarct scar. 
In view of the experimental evidence suggesting a role for mechanical, structural, and chemical cues in determining the structural properties of healing infarcts and our limited insight into how fibroblasts integrate and respond to combinations of these cues, we developed an agent-based model of infarct healing in order to test hypotheses about how environmental cues guide fibroblasts to align collagen in healing infarcts. Agent-based modeling is well-suited to addressing problems of this kind, which investigate how the behaviors of individual agents within a system give rise to emergent properties of the whole system (Simpson et al., 2007; Chavali et al., 2008; Groh \& Wagner, 2011). The work presented in this paper was primarily inspired by the dermal wound healing model published by McDougall et al. (2006). Our strategy was to build an agent-based model that incorporated experimentally measured fibroblast behaviors, environmental conditions, and parameter values in order to predict the structural properties of the collagen matrix formed during infarct healing. We then independently perturbed the mechanical, structural, and chemical guidance cues in the model and determined the importance of each cue for predicting measured collagen fiber structures in healing infarcts. Understanding the role of each cue is important for predicting how therapies that intentionally or unintentionally modify one or some combination of these cues will affect infarct scar structure and cardiac function. 


\subsection{METHODS}

\section{Model Description}

\section{Model Space and Matrix Structure}

The agent-based model of infarct healing represented a midwall section of myocardium parallel to the epicardial surface as a two-dimensional rectangular space (Fig. 2.1a,b). This space was divided into 2.5 by $2.5 \mu \mathrm{m}$ squares or patches. At each patch, a collagen fiber angle distribution and a non-collagen fiber angle distribution defined the local tissue structure (Fig. 2.1d). The non-collagen fiber angle distribution represented all components of myocardium that were neither collagen nor fibroblasts. (Since normal myocardium is highly aligned, we considered it important to capture the influence of the entire structure, not just the collagen fibers, on fibroblast alignment.) Fiber angles were divided into 5 degree bins ranging from -90 to +90 degrees. The initial fiber angle distributions were prescribed to match the structure of normal myocardium. The area within a circle or ellipse centered in the space defined the infarct region. Remodeling of collagen fiber angle distributions via degradation, deposition, and rotation was allowed throughout the model space. Remodeling of non-collagen fiber angle distributions was prohibited except for degradation within the infarct, which gradually eroded the influence of the non-collagen structure on fibroblast alignment. 


\section{Inflammation}

The concentration profile of a single, generic chemokine represented the milieu of cytokines and chemokines released by necrotic myocytes, inflammatory cells, and fibroblasts within the infarct. A two-dimensional, steady state, reaction-diffusion equation was solved using the partial differential equation solver in Matlab (The MathWorks, Inc., Natick, MA). The infarct geometry was centered in a rectangular domain ten times the size of the infarct in order to approximate diffusion into an infinite space. Concentration was set to zero at the domain boundary. Chemokine degradation was assumed to occur everywhere, while chemokine generation was restricted to the infarct region (Eqn. 2.1).

$D_{c} \nabla^{2} C_{c}=\left\{\begin{array}{r}k_{c, \text { deg }} C_{c}-k_{c, \text { gen }}, \\ k_{c, \text { deg }} C_{c}, \text { inside infarct region } \\ \text { outsinfarct region }\end{array}\right.$

$D_{c}$ - chemokine diffusion coefficient, $C_{c}$ - chemokine concentration, $k_{c, g e n}$ - chemokine generation rate coefficient, $k_{c, \text { deg }}$ - chemokine degradation rate coefficient

Chemokine concentration modulated the activation level of fibroblasts. In the agent-based model, the chemokine concentration at any location was found by linear interpolation of the discrete solution of the reaction-diffusion equation. Fibroblast migration speed, proliferation rate, collagen deposition rate, and collagen degradation rate varied linearly with chemokine concentration between minimum and maximum values representing quiescent and activated fibroblast behavior (Eqn. 2.2). 
$P_{i}(x, y)=\left(\frac{P_{i, \max }-P_{i, \min }}{C_{c, \text { max }}-C_{c, \text { min }}}\right)\left[C_{c}(x, y)-C_{c, \min }\right]+P_{i, \text { min }}$

$P_{i}-\mathrm{i}^{\text {th }}$ rate parameter (fibroblast migration speed, proliferation rate, collagen deposition rate, or collagen degradation rate), $P_{i, \max }$ - maximum rate (activated fibroblast), $P_{i, \min }-$ minimum rate (quiescent fibroblast), $C_{c, \max }$ - maximum chemokine concentration, $C_{c, \min }$ - minimum chemokine concentration, $C_{c}$ - chemokine concentration

\section{Mechanics}

Mechanical strain was modeled as a field variable. Strains in the remote myocardium were prescribed as $-5 \%$ in both the circumferential and longitudinal directions, based on experimental measurements prior to infarction in open-chest rats (Fomovsky \& Holmes, 2010). Strains in the center of the infarct were prescribed as either equibiaxial or uniaxial. For the equibiaxial case, strain was prescribed as $5 \%$ in both the circumferential and longitudinal directions in order to simulate the deformation pattern observed in cryoinfarcts located on the apex of the left ventricle in rats (Fomovsky et al., 2012b). For the uniaxial case, strain was prescribed as 5\% in the circumferential direction and $0 \%$ in the longitudinal direction in order to simulate the deformation pattern observed in cryoinfarcts located on the anterior, mid-ventricular wall (Fomovsky et al., 2012b). Between the center of the infarct and the remote myocardium, the strain profile was assumed to be mostly flat except for a relatively sharp transition at the infarct border, in agreement with systolic strains measured across the border zone of an acutely ischemic region in dog hearts (Gallagher et al., 1986; Mazhari et al., 2000). The strain field was modeled as a modified cumulative normal distribution centered at the infarct border with 
standard deviation $\sigma_{b}$ equal to $8 \%$ of the characteristic diameter of the infarct (Eqn. 2.3), as motivated by the work of Gallagher et al. (1986).

$\varepsilon_{i}(R, \theta)=\varepsilon_{i, \text { infarct }}+\left(\varepsilon_{i, \text { remote }}-\varepsilon_{i, \text { infarct }}\right) \frac{1}{\sigma_{b} \sqrt{2 \pi}} \int_{-\infty}^{R} e^{-\left[r-R_{b}(\theta)\right]^{2} /\left(2 \sigma_{b}{ }^{2}\right)} d r$

$(R, \theta)$ - polar coordinates relative to the center of the infarct, $\varepsilon_{i}$ - $\mathrm{i}^{\text {th }}$ strain component, $\varepsilon_{i, \text { inf arct }}$ - strain in the center of the infarct, $\varepsilon_{i, \text { remote }}-$ strain in the remote myocardium, $R_{b}$ - radial position of infarct border, $\sigma_{b}$ - standard deviation describing the width of the strain transition at the infarct border

Fibroblasts

Fibroblasts were represented as discs of $5 \mu \mathrm{m}$ radius (Fig. 2.1c). Fibroblasts responded to local environmental signals according to rules based on experimental observations of fibroblast behavior. Environmental signals were: chemokine concentration, strain, and fiber alignment. Behaviors were: migration, mitosis, apoptosis, collagen fiber deposition, collagen fiber rotation, and matrix degradation. Counters for each cell kept track of the time elapsed since the last cell division and the overall age of the cell. Fibroblasts could move anywhere within the model space, except they were not permitted to overlap. Fibroblast orientation was determined by the combined influence of the current cell orientation, local chemokine gradient, local strain anisotropy, and local fiber alignment. 
The model flowchart illustrates the fibroblast decision making process (Fig. 2.2). All model computations were performed using Matlab (The MathWorks, Inc., Natick, MA). The model was executed by stepping through time, and at each time point, stepping through all of the fibroblasts. At every time step, the order of stepping through the fibroblasts was randomized. Once a fibroblast reached its age limit, it was removed from the model. Once a fibroblast reached its cell cycle limit, a new fibroblast was created if there was space available adjacent to the original fibroblast. Once a fibroblast updated its direction, it migrated for a time step if there were no cells blocking its path. A fibroblast would then remodel the matrix located within its boundary by degrading matrix fibers, depositing collagen fibers, and rotating collagen fibers.

Fibroblast orientation was determined probabilistically, based on the strength of the local guidance cues: strain anisotropy (mechanical cue), fiber alignment (structural cue), chemokine gradient (chemical cue), and current fibroblast orientation (persistence cue). Each cue was represented as a vector $\vec{v}_{i}$ that could have magnitude ranging from 0 to some upper bound $M_{i}$ equal to the maximum possible value in the model. These vectors were calculated by integrating each guidance cue over the surface of the cell, as explained in Appendix 2.A. Each vector was normalized by $M_{i}$, weighted by a factor $W_{i}$, which prescribed the relative sensitivity of fibroblasts to each cue (Eqn. 2.4), and then averaged to obtain a resultant vector $\vec{\rho}$ (Eqn. 2.5, Fig. 2.3a). Note, since strain and fiber direction are both axial cues (i.e., both 0 degrees and 180 degrees represent the direction of circumferential strain or a circumferentially oriented fiber), it was necessary to consider cases where the mechanical cue vector and structural cue vector were rotated 180 degrees, and then choose the resultant vector with the largest magnitude. The angle and magnitude of the resultant vector then defined the shape of a wrapped normal distribution, 
which for directional data is analogous to a Gaussian probability density function (Eqns. 2.6-7, Fig. 2.3b). Fibroblast orientation was determined by taking a random sample from a large array of angles representative of the wrapped normal distribution. This overall formulation ensured that each individual cue could cause a high degree of fibroblast alignment, that the strength of fibroblast alignment approaches a limit as cues are added in parallel, and that no combination of cues could cause perfect fibroblast alignment.

$\vec{c}_{i}=\frac{W_{i}}{M_{i}} \vec{v}_{i}$

$\vec{\rho}=\frac{\sum \vec{c}_{i}}{\alpha+\sum\left\|\vec{c}_{i}\right\|}$

$\varphi_{w n}(\theta ; \bar{\theta}, \bar{\rho})=\frac{1}{\sigma \sqrt{2 \pi}} \sum_{k=-\infty}^{\infty} e^{-(\theta-\bar{\theta}+2 \pi k)^{2} /\left(2 \sigma^{2}\right)}, \quad-\pi<\theta \leq \pi$

$\sigma^{2}=-2 \ln \bar{\rho}$

$\vec{v}_{i}$ - un-weighted, un-normalized cue vector, $M_{i}$ - normalization factor, $\vec{c}_{i}$ - weighted and normalized cue vector, $W_{i}$ - weight factor, $\vec{\rho}$ - resultant vector, $\alpha$-persistence tuning factor which ensures that $\|\vec{\rho}\|<1, \varphi_{w n}$ - wrapped normal probability density function, $\theta$ - angle, $\bar{\theta}$ mean angle of $\vec{\rho}, \bar{\rho}$ - magnitude of $\vec{\rho}, \sigma$ - circular standard deviation

Fibroblasts remodeled extracellular matrix located within their boundary by degrading fibers, depositing fibers, and rotating fibers. Fibroblasts degraded collagen fibers at a rate determined by the local collagen concentration and local chemokine concentration (Eqn. 2.8). Fibroblasts deposited collagen fibers aligned with the current cell orientation at a rate determined by the local chemokine concentration (Eqn. 2.8). Fibroblasts rotated collagen fibers toward parallel (or 
anti-parallel if closer) alignment with the current cell orientation at a rate that decreased as fibers approached co-alignment with the cell (Eqn. 2.9) (McDougall et al., 2006). Degradation of noncollagen fibers was the same as degradation of collagen fibers, except only permitted in the infarct region (Eqn. 2.10). Deposition and rotation of non-collagen fibers were prohibited.

$\frac{d N_{c f}(\theta, t)}{d t}=k_{c f, g e n}\left(C_{c}\right) \delta\left(\theta_{c e l l}\right)-k_{c f, \operatorname{deg}}\left(C_{c}\right) N_{c f}(\theta, t)$

$\frac{d \theta_{c f}}{d t}=\left\{\begin{array}{r}k_{c f, \text { rot }}\left\|\sin \left(\theta_{c e l l}-\theta_{c f}\right)\right\|,\left(\theta_{c e l l}-\theta_{c f}\right) \text { in quadrants I and III } \\ -k_{c f, \text { rot }}\left\|\sin \left(\theta_{c e l l}-\theta_{c f}\right)\right\|,\left(\theta_{\text {cell }}-\theta_{c f}\right) \text { in quadrants II and IV }\end{array}\right.$

$\frac{d N_{n c f}(\theta, t)}{d t}=-k_{n c f, \operatorname{deg}}\left(C_{c}\right) N_{n c f}(\theta, t)$

$N_{c f}$ - number of collagen fibers located within the cell boundary, $\mathrm{t}$ - time, $k_{c f, g e n}$ - collagen fiber generation rate coefficient, $C_{c}$ - chemokine concentration, $\delta$ - delta function indicating collagen fibers are deposited only aligned with the cell orientation, $\theta_{\text {cell }}$ - current cell orientation, $k_{c f, d e g}$ - collagen fiber degradation rate coefficient, $N_{n c f}$ - number of non-collagen fibers located within the cell boundary, $k_{n c f, d e g}$ - non-collagen fiber degradation rate coefficient, $\theta_{c f}$ - collagen fiber direction, $k_{c f, r o t}$ - collagen fiber rotation rate coefficient

\section{Scaling}

The model simulated healing of a reduced scale infarct in order to shorten simulation time and decrease memory consumption, both of which were approximately proportional to model area. There were two important aspects of this model to consider when scaling: the diameter of the cells compared to the characteristic width of the chemokine gradient, and the number of patches 
covered by a single cell. First, for a fixed cell radius of $5 \mu \mathrm{m}$, we specified that at least 10 cells should fit along the width of the chemokine gradient peak. Using the dimensionless chemokine concentration profile, we calculated a minimum geometric scaling factor of 0.04 , defined as the ratio of the model infarct diameter to the experimental infarct diameter. Second, we specified that at least 10 patches should fit within the area of the cell and calculated a patch spacing of $2.5 \mu \mathrm{m}$. Since we scaled the model geometrically, the fibroblast migration speed, which was the only transport parameter (a parameter with dimensions that are some combination of length and time) governing infiltration of fibroblasts into the infarct, was multiplied by a scaling factor equal to 0.2 , defined as the ratio of the smallest dimension of the model infarct to the smallest dimension of the corresponding experimental infarct.

\section{Parameter Specification}

The model contained a total of 25 parameters (Appendix 2.B). The majority of these described the model geometry, environment, and initial conditions, and they were therefore constrained by the conditions of the experiments to be simulated. Of the remaining 12 parameters, which determined the behavior of the model, 2 parameters were estimated from literature data (fibroblast migration speed and proliferation rate), and 5 parameters were estimated by simulating published experiments and fitting model results to the published data, as described in more detail below. For the remaining 5 uncertain parameters, of which only 4 were important

for the model results $\left(k_{c f, r o t}, k_{n c f, d e g}, W_{m}\right.$, and $\left.W_{c}\right)$, conservative estimates were chosen. For example, weight factors for the mechanical and chemical guidance cues were set equal to the structural cue weight factor, which was estimated from experimental data. 
The persistence cue weight factor $W_{p}$, structural cue weight factor $W_{s}$, and persistence tuning constant $\alpha$ were estimated by matching simulated migration behavior to published measurements. Dickinson et al. (1994) measured the migration tracks of fibroblasts in collagen gels with low and high degrees of collagen fiber alignment, calculated mean squared displacement curves for each condition, fit the data to the persistent random walk model of cell migration, and reported random migration coefficients for $\mathrm{x}$ - and $\mathrm{y}$-directed motion. We used our agent-based model to simulate 10,000 migration tracks of fibroblasts in unaligned or aligned collagen matrices, performed the same analysis described by Dickinson et al., and adjusted $W_{p}$, $W_{s}$, and $\alpha$ to fit the random migration coefficients derived from the simulations to the published values. The simulated migration behavior was determined by the ratios $\alpha / W_{p}$ and $W_{s} / W_{p}$, such that $W_{p}$ could be arbitrarily chosen. The values $\alpha / W_{p}=0.53$ and $W_{s} / W_{p}=0.5$ provided the best match to the published migration coefficients (Fig. 2.4).

Collagen fiber degradation and deposition rate coefficients were estimated by fitting published measurements of collagen accumulation in healing infarcts. Fomovsky and Holmes (2010) measured collagen area fraction in rat ligation infarcts after 1,2,3, and 6 weeks of healing. We estimated collagen degradation and deposition rate coefficients by fitting the reported collagen accumulation time course to an ordinary differential equation accounting for zeroth order deposition and first order degradation of collagen (Eqns. 2.11-13). We also included a time lag to account for the approximately 3 day delay before fibroblasts appear in large numbers at the site of the infarct. The values $k_{c f, \text { deg }}=0.0025 h r^{-1}$ and $k_{c f, g e n}=0.073 \%$ area $/ h r$ provided the best match to the published collagen accumulation time course. Finally, we verified that the 
fitted parameter values enabled the agent-based model to reproduce the experimental data (Fig. 2.5).

$\frac{d F_{c f}}{d t}=k_{c f, g e n}^{\prime}-k_{c f, d e g}^{\prime} F_{c f}$

$F_{c f}(t)=\left(F_{c f, o}-F_{c f, \infty}\right) e^{-k_{c f, d e g}^{\prime}\left(t-t_{o}\right)}+F_{c f, \infty}$

$k_{c f, g e n}^{\prime}=k_{c f, \operatorname{deg}}^{\prime} F_{c f, \infty}$

$F_{c f}$ - collagen fiber area fraction, $\mathrm{t}-$ time, $k_{c f, g e n}^{\prime}$ - collagen fiber generation rate coefficient, $k_{c f, d e g}^{\prime}$ - collagen fiber degradation rate coefficient, $F_{c f, o}$ - collagen fiber area fraction at time $t_{o}, F_{c f, \infty}$ - collagen fiber area fraction at steady state

\section{Simulations and Analysis}

Infarcts with different mechanical conditions (equibiaxial or uniaxial strain), and different shapes (circumferentially oriented ellipse, circle, longitudinally oriented ellipse) were modeled in order to cover the range of conditions investigated in the cryoinfarction study performed by our lab (Fomovsky et al., 2012b). In order to test the effect of the mechanical guidance cue on the predicted collagen structure, a subset of simulations were run with mechanosensing turned off by setting the weight factor for the mechanical cue equal to zero. In order to test the effect of the structural guidance cue on the predicted collagen structure, simulations were run with the fibers of the initial matrix structure oriented randomly, assigned a medium-high level of circumferential alignment equal to that of mid-thickness native myocardium, or perfectly aligned in the circumferential direction. In order to test the effect of rapid matrix degradation during the 
initial infarction, simulations were run with the initial matrix density set equal to or $1 / 10^{\text {th }}$ the density of normal myocardium. Finally, in order to examine the balance between structural and mechanical cues at transmural locations other than mid-depth, 2D infarct layers were modeled with the mean fiber angle of the initial matrix structure varying from -60 to +60 degrees relative to the circumferential axis, corresponding to the fiber angle variation observed through the thickness of normal myocardium.

Models were run to simulate 6 weeks of healing (Fig. 2.6). Time courses of strength of collagen alignment, quantified as mean vector length, were compared among different model conditions. Predicted infarct collagen orientation histograms at the 3 week time point were compared to in vivo measurements taken 3 weeks after cryoinfarction in rats (Fomovsky et al., 2012b). Predicted transmural profiles of mean collagen fiber angle and strength of collagen alignment were compared to in vivo measurements taken 3 weeks after ligation of an obtuse marginal branch of the left circumflex coronary artery in pigs (Holmes, 1995; Holmes et al., 1997).

\section{Parameter Sensitivity Analysis}

A parameter sensitivity analysis was performed in order to determine which sets of model parameters have influence over each of several model predictions of interest. Circular infarcts experiencing uniaxial strain were modeled. Descriptive parameters investigated were: initial collagen matrix density $\left(\mathrm{F}_{\mathrm{cf}, \mathrm{i}}\right)$, initial non-collagen matrix density $\left(\mathrm{F}_{\mathrm{ncf}, \mathrm{i}}\right)$, initial strength of alignment of collagen fibers $\left(\mathrm{MVL}_{\mathrm{cf}, \mathrm{i}}\right)$, and initial strength of alignment of non-collagen fibers $\left(\mathrm{MVL}_{\mathrm{ncf}}\right)$. Behavioral parameters investigated were: collagen degradation rate coefficient 
$\left(\mathrm{k}_{\mathrm{cf}, \mathrm{deg}}\right)$, non-collagen degradation rate coefficient $\left(\mathrm{k}_{\mathrm{ncf}, \mathrm{deg}}\right)$, collagen generation rate coefficient $\left(\mathrm{k}_{\mathrm{cf}, \mathrm{gen}}\right)$, collagen rotation rate coefficient $\left(\mathrm{k}_{\mathrm{cf}, \mathrm{rot}}\right)$, cell migration speed $\left(\mathrm{S}_{\text {cell }}\right)$, cell cycle duration (time to mitosis, $\left.\mathrm{T}_{\mathrm{m}}\right)$, mechanical cue weight factor $\left(\mathrm{W}_{\mathrm{m}}\right)$, structural cue weight factor $\left(\mathrm{W}_{\mathrm{s}}\right)$, and chemical cue weight factor $\left(\mathrm{W}_{\mathrm{c}}\right)$. Model predictions of interest were: collagen fiber accumulation time course, cell accumulation time course, collagen fiber alignment time course, and cell alignment time course. From the baseline set of model parameters that yielded predictions in good agreement with experimental data (Appendix 2.B), models were run with each parameter independently perturbed. Most of the parameters were scaled by a factor of $1 / 10$, $1 / 4,1 / 3.16,1 / 2,2,3.16,4$, or 10 . Initial fiber mean vector length (strength of fiber alignment) was adjusted to $0.1,0.3,0.5,0.6,0.7,0.8$, or 0.9 because mean vector length is bounded from 0 to 1 .

Models were run to simulate 6 weeks of healing. Time courses of strength of collagen fiber alignment, strength of cell alignment, collagen matrix density, and cell concentration within the infarct were computed. Model predictions at early (3d), intermediate (1wk), and late (3wk) time points were selected for sensitivity analysis. For each input parameter and output prediction, a ratio was calculated as the perturbed model value divided by the baseline model value (Eqn. 2.14a, b). Relationships between model outputs and model inputs were visualized by plotting model output ratios versus model input ratios on log-log axes (Fig. 2.7). On these plots, a flat line at a value of 1 indicated the output was unaffected by changing the input, while deviation of points away from this flat line indicated the output was affected by changing the input. Sensitivities were calculated as the slope of the log-transformed data. A slope of 1 indicated a 
proportional relationship between model input and output. A slope of -1 indicated an inversely proportional relationship. Slopes close to 0 indicated no relationship.

$M I R=($ perturbed model input value $) /($ baseline model input value $)$

$M O R=($ perturbed model output value $) /($ baseline model output value $)$

$M I R$ - model input ratio, $M O R$ - model output ratio

Experimental data were used to define a sensitivity threshold. A model output was assumed to be significantly changed if it shifted more than one standard deviation away from the baseline output. Experimental data were available for the collagen mean vector length at 3 weeks $(0.43$ $+/-0.165)$, collagen area fraction at 1 week $(9+/-4)$, and collagen area fraction at 3 weeks $(21$ +/- 9). Lower and upper threshold values for the model output ratios were calculated from the experimental data (Eqn. 2.15a, b). Since these lower and upper threshold values were similar for all three cases $(\sim 0.58$ and $\sim 1.42)$, they were averaged and assumed to be applicable to all of the model outputs. A threshold level of the sensitivity (slope of log-transformed data) was calculated to be 0.194 , using 10 and 0.1 as the upper and lower bounds of the model input ratios (Eqn. 2.16). If a sensitivity value was greater than this threshold, then an order of magnitude change in the model input caused the model output to shift more than $42 \%$. For these cases, upper and lower bounds of the model input ratio that would keep the model output ratio between 0.58 and 1.42 were interpolated from the model output ratio versus model input ratio curves. 
$M O R_{U T}=\left(\mu_{\text {expt }}+\sigma_{\text {expt }}\right) / \mu_{\text {expt }}$

$M O R_{L T}=\left(\mu_{\text {expt }}-\sigma_{\text {expt }}\right) / \mu_{\text {expt }}$

Sensitivity Threshold $=\frac{\left(\log _{10} M O R_{U T}-\log _{10} M O R_{L T}\right)}{\left(\log _{10} 10-\log _{10} 0.1\right)}$

$M O R_{U T}$ - upper threshold of the model output ratio, $M O R_{L T}$ - lower threshold of the model output ratio, $\mu_{\text {expt }}$-experimentally measured mean of output parameter, $\sigma_{\text {expt }}$-experimentally measured standard deviation of output parameter

\subsection{RESULTS}

\section{Chemical Guidance Cue}

The chemical guidance cue had little effect on the predicted collagen alignment for the range of shapes examined in our cryoinfarct study, which was consistent with the experimental data. Model simulations were run with: longitudinally oriented elliptical infarcts, circular infarcts, or circumferentially oriented elliptical infarcts; circumferentially oriented initial matrix, as seen in normal myocardium at mid-thickness; and uniaxial strain in the circumferential direction, as measured in anterior, mid-ventricular cryoinfarcts. The different shapes created different spatial patterns of the chemokine gradient. However, the model predicted only small differences in collagen alignment that were well within the error of the experimental measurements from the mid-ventricular cryoinfarcts, consistent with the lack of an effect of infarct shape on collagen alignment in our cryoinfarct study (Fig. 2.8a). We then tested for conditions where the model 
would predict a measurable difference in the strength of collagen alignment between circumferentially and longitudinally oriented, elliptical infarcts. The infarcts would need an aspect ratio of at least 10:1 or the fibroblasts would need to be at least 4-fold more sensitive to the chemical cue in order for the orientation of the elliptical infarct to measurably affect the strength of collagen alignment (Fig. 2.8b).

\section{Structural Guidance Cue}

The initial matrix structure had little effect on the predicted collagen alignment after several weeks of healing, which was consistent with data from experimental models of infarction. First, model simulations were run with: circular infarcts; the initial matrix structure either perfectly aligned in the circumferential direction or unaligned; and uniaxial strain in the circumferential direction, as measured in circular, mid-ventricular cryoinfarcts. The initial matrix alignment had an effect on the alignment of deposited collagen at early times, but the predictions for the two different initial structures gradually converged over time (Fig. 2.9a). By three weeks, the predicted collagen fiber orientation histograms fell within a standard deviation of the experimental mean of the mid-ventricular cryoinfarct data, regardless of the strength of alignment of the initial matrix structure (Fig. 2.9b). Similarly, when the initial matrix density was decreased to $1 / 10^{\text {th }}$ the density of normal myocardium, the model predicted lower alignment of collagen in the infarct at early times, but this difference became small after 3 weeks of healing (Fig. 2.9a). Finally, the model was used to simulate infarct healing at different transmural depths by prescribing different initial mean fiber angles ranging from -60 to +60 degrees. Again, the predicted infarct collagen structures gradually converged over time (Fig. 2.10a,b). By three 
weeks, the predicted collagen fiber angle gradient through the thickness of the infarct was much smaller than the initial gradient (Fig. 2.10c), while the strength of collagen alignment was slightly higher at mid-thickness than at the epicardial and endocardial surfaces (Fig. 2.10d). These predictions were consistent with the transmural trends observed in pig infarcts (Fig. 2.10e,f), which also experienced transmurally uniform, uniaxial stretch in the circumferential direction.

\section{Mechanical Guidance Cue}

A mechanical guidance cue was required to support long-term alignment of collagen in infarct scar, which was consistent with the experimental cryoinfarction data. Models were run with: circular infarcts; circumferentially oriented initial matrix; and either uniaxial strain in the circumferential direction or equibiaxial strain. For each mechanical condition, model simulations were run with mechanosensing turned on or off. With mechanosensing on, if the pattern of stretch was equibiaxial, the initially aligned matrix was replaced by randomly oriented collagen, whereas if the pattern of stretch in the infarct was uniaxial, scar formed with aligned collagen fibers (Fig. 2.11a). The predicted collagen fiber orientation histograms after 3 weeks of healing were consistent with those observed for apical cryoinfarcts that experienced biaxial stretch (Fig. 2.11b) and mid-ventricular cryoinfarcts that experienced uniaxial stretch (Fig. 2.11c). In contrast, when mechanosensing was turned off, although the model results for the case of equibiaxial stretch were unchanged (Fig. 2.11b), the model failed to reproduce the collagen fiber alignment seen in the uniaxially stretched cryoinfarcts (Fig. 2.11c). 


\section{Parameter Sensitivity Analysis}

The strength of cell alignment was sensitive to the mechanical and structural cue weight factors at every time point. The strength of cell alignment was also sensitive to the strength of noncollagen fiber alignment early after infarction, but this sensitivity decreased over time (Fig. 2.12a, Table 2.1).

The concentration of cells in the infarct was sensitive to the cell cycle duration at every time point. The concentration of cells was also sensitive to the cell speed and chemical cue weight factor early after infarction, but this sensitivity decreased over time (Fig. 2.12b, Table 2.1).

The strength of collagen fiber alignment in the infarct was sensitive to the initial strength of collagen fiber alignment early after infarction, but this sensitivity decreased over time, while sensitivity to the mechanical and structural cue weight factors increased over time. The strength of collagen fiber alignment was sensitive to the initial collagen matrix density and collagen fiber generation rate coefficient at the intermediate time point. The strength of collagen fiber alignment was also sensitive to the collagen fiber rotation rate coefficient and strength of noncollagen fiber alignment at the late time point (Fig. 2.12c, Table 2.1).

The collagen matrix density was sensitive to the collagen fiber generation rate coefficient at every time point, while sensitivity to the collagen fiber degradation rate coefficient increased over time. The collagen matrix density was also sensitive to the initial collagen matrix density early after infarction, but this sensitivity decreased over time (Fig. 2.12d, Table 2.1). 
For each of the sensitive input/output relationships listed in Table 2.1, upper and lower bounds of the model input ratio that would keep the model output within $+/-42 \%$ of the baseline value were calculated. This information was then simplified to a list of upper and lower bounds for each input parameter that would ensure every model output remained within $+/-42 \%$ of baseline (Table 2.2). The highest MIR lower bound on this list was 0.51 and the lowest MIR upper bound was 1.3. On average, these input parameters could be off by a factor of $1 / 2.5$ to a factor of 2.5 without appreciably changing the model predictions.

\subsection{DISCUSSION}

Prior studies have proposed that mechanical, structural, or chemical cues could regulate the anisotropy of infarct scar tissue. We developed an agent-based model that accounted for the combined influence of these cues on fibroblast alignment, collagen deposition, and collagen remodeling. We pooled published experimental data from several sources in order to determine parameter values in the model (Appendix 2.B), and then tested model predictions against collagen alignment measurements from a set of recent cryoinfarction experiments that independently varied infarct shape and pattern of stretch in a consistent animal model (Fomovsky et al., 2012b). The model predicted collagen structures in very good agreement with the experimental measurements across all cryoinfarct groups. We then independently perturbed the mechanical, structural, and chemical cues in the model in order to determine how strongly each cue influenced the predicted collagen structure. Although chemokine gradients and preexisting matrix structures influenced spatial and temporal patterns of collagen organization in 
certain situations, a mechanical cue was critical to reproducing the collagen alignment observed experimentally in healing infarcts.

\section{Chemical Guidance Cue}

McDougall et al. (2006) proposed that the pattern of collagen alignment in dermal wounds could be explained by alignment of fibroblasts in the direction of the chemical gradient established by chemokines generated within the wound. According to this hypothesis, differently shaped infarcts should have different chemokine gradient patterns and different patterns of collagen alignment. We used our model to simulate healing of a longitudinally oriented elliptical infarct, circular infarct and circumferentially oriented infarct, and found a negligible effect of infarct shape on the average collagen alignment (Fig. 2.8a). Though in contrast with the model results of McDougall et al., this finding was consistent with our cryoinfarction data. The model provides some insight as to why this is the case. The predicted chemokine concentration profile was mostly flat throughout the bulk of the infarct and decreased sharply at the infarct border. Consequently, the influence of the chemical cue on collagen fiber alignment was limited to the region near the infarct border where the steep concentration gradient greatly increased the strength of the chemical guidance cue. Only in model infarcts with very high aspect ratios (>10:1) did this border effect measurably influence the average collagen fiber alignment (Fig. 2.8b). Although we restricted this study only to circular and elliptical infarcts, the results imply that chemokine gradients might explain some regional variation in the collagen structure of ligation infarcts, which tend to be more irregularly shaped than the cryoinfarcts of interest in this work. 


\section{Structural Guidance Cue}

Zimmerman et al. (2000) proposed that the orientation of collagen fibers in healing infarcts could be explained by the orientation of the matrix surrounding native myocardium, some of which survives and acts as a template for alignment of fibroblasts and collagen during scar formation. We used our model to simulate healing of an infarct with an initial matrix structure that was either perfectly aligned or unaligned (random), and found that the influence of the initial matrix structure on the predicted collagen alignment faded over the time course of healing (Fig. 2.9). We also used our model to simulate healing of an infarct with mechanosensing turned off, and found that an initially aligned matrix structure alone was unable to support long-term collagen alignment (Fig. 2.11c). These results were consistent with our cryoinfarction experiments, which found that alignment of collagen fibers in infarct scar was only seen in infarcts that experienced uniaxial stretch. The model provides some insight as to why the initially aligned matrix structure failed to support long term collagen alignment in healing infarcts. In the model, the choice of fibroblast orientation had an element of randomness, which ensured that fibroblasts would not always orient perfectly in the direction of the initially aligned matrix fibers. Since fibroblasts deposited collagen fibers along and rotated collagen fibers toward their current orientation, the strength of alignment of the matrix was therefore reduced by the imperfectly aligned cells. Subsequently, fibroblasts would have an even lesser tendency to align with the initial structural cue. In other words, since fibroblasts can change the matrix structure, then it cannot be a stable guidance cue. Interestingly, we recently reported that collagen fiber distributions in scars that experienced biaxial stretch were random by 1 week after 
coronary ligation in the rat (Fomovsky \& Holmes, 2010), suggesting that our current model actually overestimates the true influence of pre-existing matrix on collagen fiber orientation in the developing scar. This could reflect the fact that some of the pre-existing matrix is degraded rapidly in the first hours following infarction (Takahashi et al., 1990). Consistent with this possibility, when we simulated infarcts with a lower initial matrix density, the model predicted a faster decrease in the strength of collagen alignment in the first few days after infarction (Fig. 2.9a). Alternatively, the weighting of structural cues could be too high in our current model. However, this seems less likely given the close agreement of experimental data with the predicted transmural trends of infarct collagen alignment, which depended on the magnitude of the structural cue weight factor (Fig. 2.10c-f).

\section{Mechanical Guidance Cue}

In our cryoinfarction study (Fomovsky et al., 2012b), we found that the location of the infarct determined its pattern of deformation, and this deformation pattern was correlated with collagen fiber alignment. When we simulated healing of an infarct with mechanosensing turned on, we found that the mechanical environment (uniaxial or equibiaxial stretch) determined whether the healing infarct ultimately formed an aligned or disorganized structure (Fig. 2.11a). This result was consistent with the experimental cryoinfarct findings. Cryoinfarcts that experienced uniaxial stretch developed scars with collagen fibers aligned in the direction of stretch, while cryoinfarcts that experienced biaxial stretch developed scars with poorly aligned collagen fibers. When mechanosensing was turned off, the collagen alignment observed in the mid-ventricular cryoinfarcts could not be reproduced (Fig. 2.11c). The model provides some insight as to why 
the mechanical cue was the most important determinant of infarct collagen alignment. First, unlike the chemical cue, which has its influence spatially restricted to the infarct border where the chemokine gradient is highest, the mechanical cue has influence throughout the bulk of the infarct. Second, unlike the structural cue, which is unstable in the absence of any supporting cues, the mechanical cue is maintained by the external loads acting on the infarct. Indeed, infarct strains were shown to be fairly consistent for six weeks after coronary ligation in rats (Fomovsky \& Holmes, 2010).

\section{Broader Comparison to Experimental Data}

Our primary source of comparison data in this paper was a set of rat cryoinfarction experiments performed in our laboratory. Although the cryoinjury model of infarction has many advantages, such as enabling control of infarct shape and location, tissue damage caused by freezing is not identical to damage caused by ischemia, and there may be important differences in the subsequent scar formation process. We therefore performed additional comparisons to published data on collagen fiber structure in healing myocardial infarcts induced by coronary ligation. Broadly, the published data agree well with our model predictions. For example, infarcts resulting from ligation of an obtuse marginal branch of the left circumflex coronary artery in pigs stretched circumferentially and developed circumferentially aligned collagen fibers (Holmes et al., 1997), while infarcts resulting from left coronary artery ligation in rats stretched biaxially and developed randomly aligned collagen fibers (Fomovsky \& Holmes, 2010). When reviewing published data on infarct collagen fiber structure, we recognized that as in our prior studies in pigs (Holmes et al., 1997), multiple investigators have reported less transmural variation of the 
collagen fiber orientation in healing infarcts than typically observed in normal myocardium (Whittaker et al., 1989; Omens et al., 1997). We therefore simulated infarct healing at different transmural depths and compared the predicted transmural profiles of mean fiber angle and mean vector length to our experimental data obtained from 3 week old ligation infarcts in pigs (Fig. 2.10 ), in which the initial muscle fiber angle varies from -60 to +60 degrees but the infarct experiences uniaxial stretch of similar magnitude at all transmural depths. Despite the fact that many of the model parameter values were derived from experimental studies of rats, the model reproduced the trends seen in the pig data strikingly well and provided an explanation for the observed structures. At mid-depth, where the mean angle of the initial matrix structure and the direction of greatest stretch coincided, the healing infarcts developed highly aligned collagen oriented in the circumferential direction. At the endocardium and epicardium, where the initial matrix structure and mechanical environment provided conflicting cues, the effect of the initial matrix structure gradually faded, so that by 3 weeks the mean collagen fiber angles in these layers were closer to circumferential but the strength of alignment was still lower than at middepth. The findings of these ligation infarction studies are consistent with the model predictions and the cryoinfarction study, and support the generality of the conclusions drawn from the model.

\section{Parameter Sensitivity Analysis}

Overall, the parameter sensitivity analysis revealed several key features of the model. First, initial conditions, such as the initial collagen matrix density and initial strength of collagen fiber alignment, had diminishing influence over time. Second, except for trivial comparisons (e.g. 
between the predicted collagen matrix density at Day 3 and the initial collagen matrix density), sensitivity values were less than 1 , indicating changes to model inputs caused less-thanproportional changes to model outputs. Both of these observations indicate that order of magnitude estimates of input parameter values should suffice where experimental data is lacking. Third, by three weeks after infarction, there was very little co-dependence of the model predictions on model inputs. For example, the predicted collagen matrix density was not sensitive to any of the same model inputs as the predicted cell concentration. The only codependence was between the strength of cell alignment and strength of collagen fiber alignment, which were both sensitive to the structural and mechanical cue weight factors. This was expected since fibroblast orientation guides collagen orientation in the model. Fourth, sensitivity of the long term strength of collagen fiber alignment to inputs other than the weight factors (e.g. collagen fiber rotation rate coefficient and strength of non-collagen fiber alignment) was relatively weak. Thus, our uncertainty in the values of these inputs does not challenge our conclusions about the relative effects of structural and mechanical cues on collagen fiber alignment during infarct healing. We discuss several insights in more detail in the following paragraphs.

Sensitivity of cell concentration to migration speed decreased over time, which indicates that movement of a front of proliferating cells (at the baseline proliferation rate) could compensate for a slow migration speed (Fig. 2.12b, Table 2.1). Interestingly, although the cell concentration was sensitive to the chemical cue weight factor, proliferation rate, and migration speed, the collagen matrix density was relatively insensitive to them (Fig. 2.12d, Table 2.1). Since accumulation of collagen in the infarct depends on both fibroblast infiltration and collagen 
deposition, this indicates that collagen deposition was the rate limiting step in the baseline model. Furthermore, if rapid accumulation of collagen is deemed a desirable therapeutic outcome, this suggests that increasing the rate of infiltration of fibroblasts into the infarct will provide little benefit. However, Laeremans et . al. (2011) recently demonstrated that blocking Frizzled signaling after infarction increased the density of fibroblasts in the infarct, actually decreased the collagen area fraction, and mitigated loss of function, which suggests that increasing the fibroblast infiltration rate or fibroblast density in an infarct could have benefits unrelated to collagen accumulation.

Sensitivity of the strength of collagen fiber alignment to the two processes of collagen fiber remodeling (aligned deposition and fiber rotation) was time dependent (Fig. 2.12c, Table 2.1). One week after infarction, collagen was rapidly accumulating, the proportion of newly deposited collagen was large relative to the proportion of existing collagen, and the strength of collagen fiber alignment was strongly affected by the deposition of aligned fibers. Three weeks after infarction, the rate of collagen accumulation slowed, the proportion of newly deposited collagen was small relative to the proportion of existing collagen, and the strength of collagen fiber alignment was strongly affected by the re-orientation of existing fibers. This implies that the effectiveness of any intervention that seeks to control the alignment of the infarct collagen matrix will depend on the timing of the intervention, which will in turn depend on which collagen remodeling processes actually occur during infarct healing in vivo. We address this topic further in Chapter 3. 
Although the collagen matrix density was sensitive to the collagen fiber generation rate coefficient at every time point, it was sensitive to the collagen fiber degradation rate coefficient only at the late time point (Fig. 2.12d, Table 2.1). Again, this is explained by the fact that collagen accumulates rapidly during the first few weeks after infarction and more slowly by three weeks after infarction. During the phase of rapid collagen accumulation, the collagen degradation rate was much smaller than the collagen generation rate and had little effect on the net amount of collagen deposited. As the rate of collagen accumulation slowed, the collagen degradation rate became similar to the collagen generation rate and had similar effect on the collagen matrix density. This implies that any intervention that seeks to control collagen density during the phase of rapid collagen accumulation after infarction should do so by altering the rate of collagen generation rather than altering the rate of collagen degradation. This may explain, at least in part, why the PREMIER trial found no benefit of MMP inhibition within 90 days after myocardial infarction (Hudson et al., 2006), and why MMP inhibition appears to have the expected effect of increasing collagen content in remote myocardium but not in the infarct (Yarbrough et al., 2003).

The most important determinants of the structure of mature $(3 \mathrm{wk})$ scar were $\mathrm{W}_{\mathrm{m}}, \mathrm{W}_{\mathrm{s}}, \mathrm{k}_{\mathrm{cf} \text {,gen }}$, $\mathrm{k}_{\mathrm{cf}, \mathrm{deg}}$, and $\mathrm{k}_{\mathrm{cf}, \text { rot }}$ (Table 2.2). Experimental measurements of collagen area fraction, strength of collagen fiber alignment, and strength of cell alignment constrain the range of values these input parameters may take. Collagen area fraction and strength of collagen fiber alignment have been measured, while strength of cell alignment has not. Thus, the strength of cell alignment must be measured in order to fully constrain the set of input parameters. Our sensitivity analysis could then be used to provide the first prediction of the range of values the undetermined parameters 
$\left(\mathrm{W}_{\mathrm{m}}, \mathrm{W}_{\mathrm{s}}, \mathrm{k}_{\mathrm{cf}, \mathrm{rot}}\right)$ might take, which could then be validated with additional experimentation. We address this topic further in Chapter 3.

\section{Model Innovations}

The agent-based model presented in this work is a significant advance in the use of computational modeling to understand infarct healing. Previous models of infarct healing have used systems of ordinary differential equations to predict the dynamics of fibroblast, macrophage, chemokine, and collagen accumulation in the infarct (Wang et al., 2010; Jin et al., 2011). More general wound healing models have used agent-based or continuum methods to predict the spatial and temporal evolution of cell populations, chemokines, and extracellular matrix components (Mi et al., 2007; Javierre et al., 2009). Several of these models were developed to predict structural remodeling of the extracellular matrix, which motivated our modeling approach (McDougall et al., 2006; Ohsumi et al., 2008; Groh \& Wagner, 2011). However, our model is the first to include the combined influence of mechanical, structural, and chemical guidance cues on fibroblast behavior to predict collagen fiber alignment in scar tissue formed after myocardial infarction. Furthermore, we carefully tied behaviors and parameter values in our model to experimental observations, which allowed the model predictions to be quantitatively compared to in vivo measurements. 


\section{Model Limitations}

Several limitations of the model highlight features of infarct healing that are currently poorly understood and merit further study. First, our model represents a $2 \mathrm{D}$ slice through the circumferential-longitudinal plane of the myocardium. Consequently, in the model, fibroblasts can only enter the infarct by migrating in this plane, whereas in vivo, cells might also infiltrate the infarct from the epicardial and endocardial borders, originate from circulating cells that enter the infarct through the vasculature, or even survive the initial infarction. Our model predicts a time course of cell infiltration that is similar to in vivo observations (1-2 weeks for cells to reach the core of the necrotic region) (Fig. 2.4), which generally supports our approach, but additional studies on the detailed pattern of fibroblast migration into the infarct are needed. Furthermore, the $2 \mathrm{D}$ model simplifies the $3 \mathrm{D}$ nature of strain and fiber angle distributions seen in normal myocardium. While we investigated the effect of the transmural gradient of initial fiber angle (Fig. 2.10), transmural gradients in strain were not considered since they have been shown to become small within minutes after infarction (Villarreal et al., 1991) and remain small through at least 3 weeks of healing (Holmes et al., 1994). Consequently, we treated the infarct as a stack of 2D layers, each with a different initial matrix structure, but all with the same mechanical environment.

Second, our model represents infarct strains as static field variables. In reality, the balance between increased stresses due to LV dilation and infarct thinning, and increased stiffness due to collagen deposition will determine how infarct strains evolve over time. Our model assumption was based on the fact that we saw little change in strain magnitudes over time in our previous 
study of healing rat infarcts (Fomovsky \& Holmes, 2010), but this cannot be assumed to hold in general. We address this topic further in Chapter 6, where we introduce an approach to coupling a finite element model to the agent-based model in order to update strains as the infarct structure changes.

Third, our model represents the chemokine concentration as a static field variable. In reality, many chemokines are released at the site of an infarct in a complex spatial and temporal pattern. Since it is not fully understood how all of these factors regulate fibroblast behavior, we chose to model a single generic chemokine, giving it the effect of "activating" fibroblast proliferation, migration, and collagen remodeling. Although representing the exact spatiotemporal profile of chemokine release is likely important for making predictions about infarct healing at early time points, we do not expect it to be important after fibroblasts have uniformly populated the infarct.

Fourth, our model represents the matrix deforming traction forces that fibroblasts generate as rotation of collagen fibers toward co-alignment with the cell. The rotation rate coefficient, which is an important determinant of the predicted time course of collagen alignment, is an unconstrained parameter in the model. We conservatively chose a value of only a few degrees per hour, and found good agreement of the model with experimental data after three weeks of healing, but data at additional time points will be needed in order to better establish a value for the rotation rate coefficient. We investigate the plausibility and limitations of the rotation process of collagen fiber remodeling in Chapter 3. 


\subsection{CONCLUSION}

Our results suggest that although chemokine gradients and pre-existing matrix structures have important effects on collagen organization, a mechanical cue is critical for the development of collagen alignment in infarct scar. It is important to understand the effects of these cues if interventions are to be designed that seek to preserve cardiac function after infarction by controlling the anisotropy of infarct scar. Furthermore, many proposed therapies for myocardial infarction, such as injection of cells or polymers (Christman et al., 2004), implantation of materials for structural support (Kelley et al., 1999), and peri-infarct pacing (Chung et al., 2010), alter the mechanics of the infarct region. Our modeling results suggest that such therapies could unintentionally change the anisotropy of the healing infarct, which could have important functional consequences. This model represents a potentially powerful tool for predicting how such interventions change healing outcomes. This work is also relevant for tissue engineering and for repair of any tissue where anisotropy is an important determinant of tissue function, such as tendons and ligaments.

\section{ACKNOWLEDGMENTS}

Many thanks to Gregory Fomovsky, Ph.D. for helpful discussions and for providing the experimental cryoinfarct data used for comparisons in this study. 


\subsection{FIGURES AND TABLES}

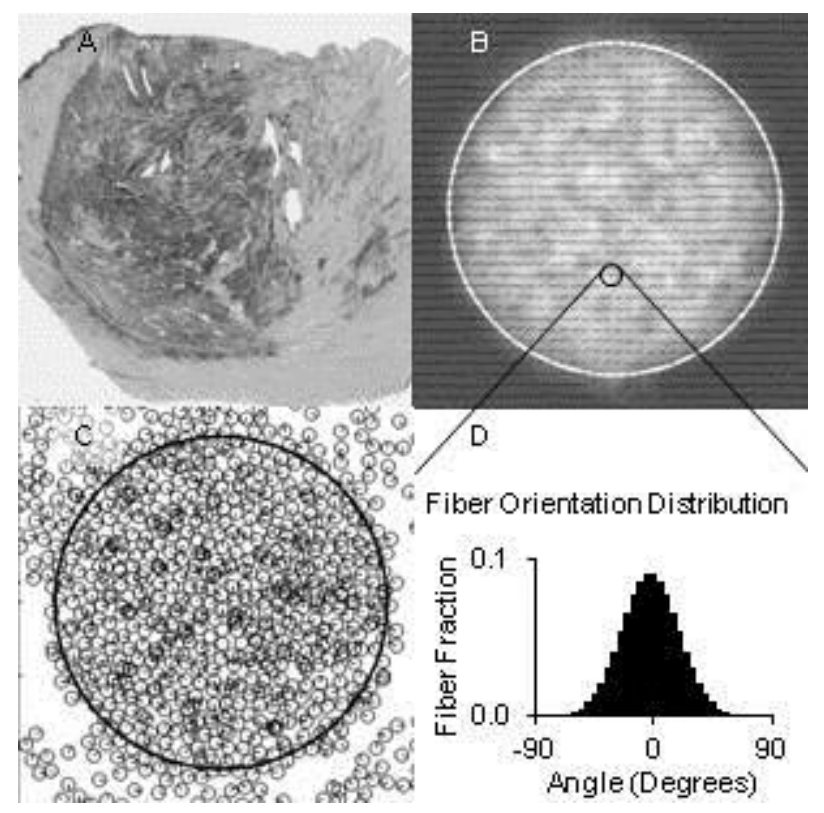

Figure 2.1. Overview of the agent-based model of infarct healing. A. Histological section of a cryoinfarct scar after 3 weeks of healing, showing picrosirius red stained collagen fibers (dark tissue). B. Example of a model result simulating the cryoinfarction experiment. Grayscale indicates collagen density and dashes indicate collagen fiber orientation. C. Example of fibroblast agents that have infiltrated the infarct region by migrating and proliferating. D. Example of local fiber orientation histogram. Each dash depicted in $(\mathbf{B})$ indicates the mean angle and mean vector length (strength of alignment) of the local fiber orientation distribution. 


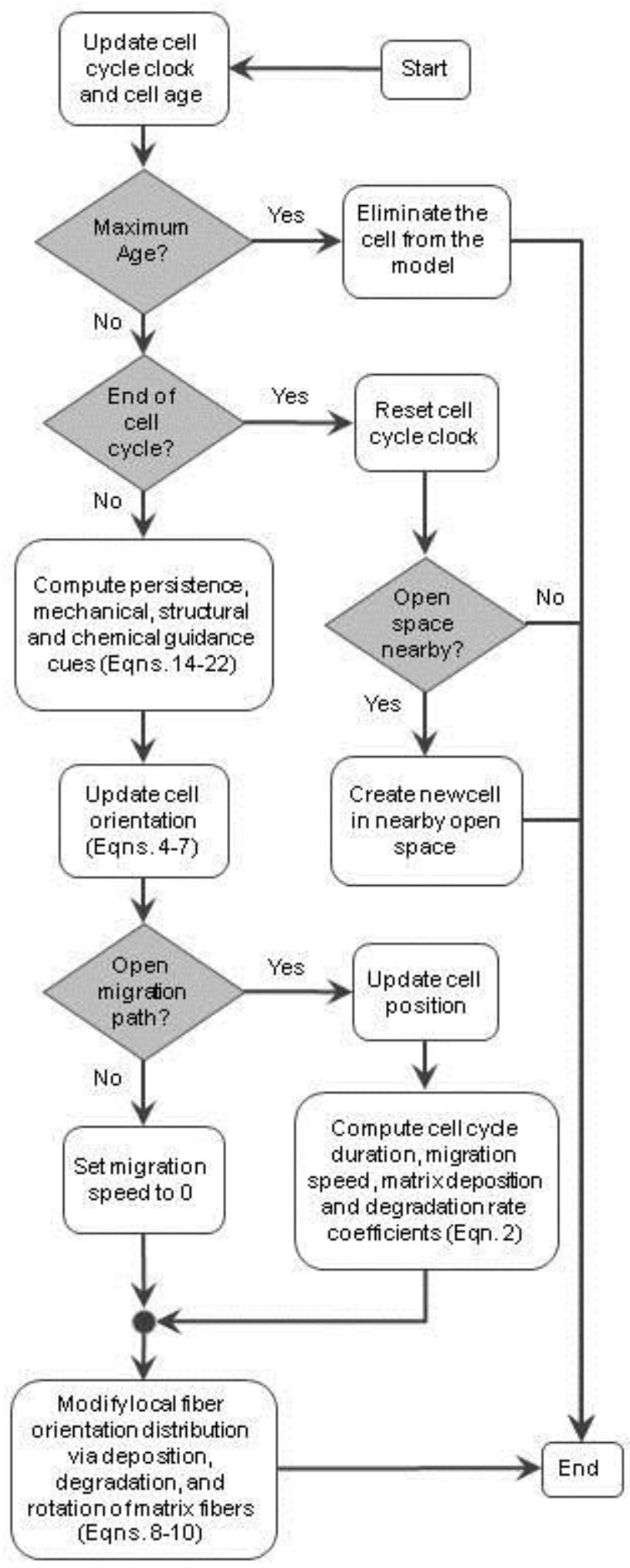

Figure 2.2. Fibroblast decision making flowchart. At every time step, every fibroblast executes these operations and decisions. 

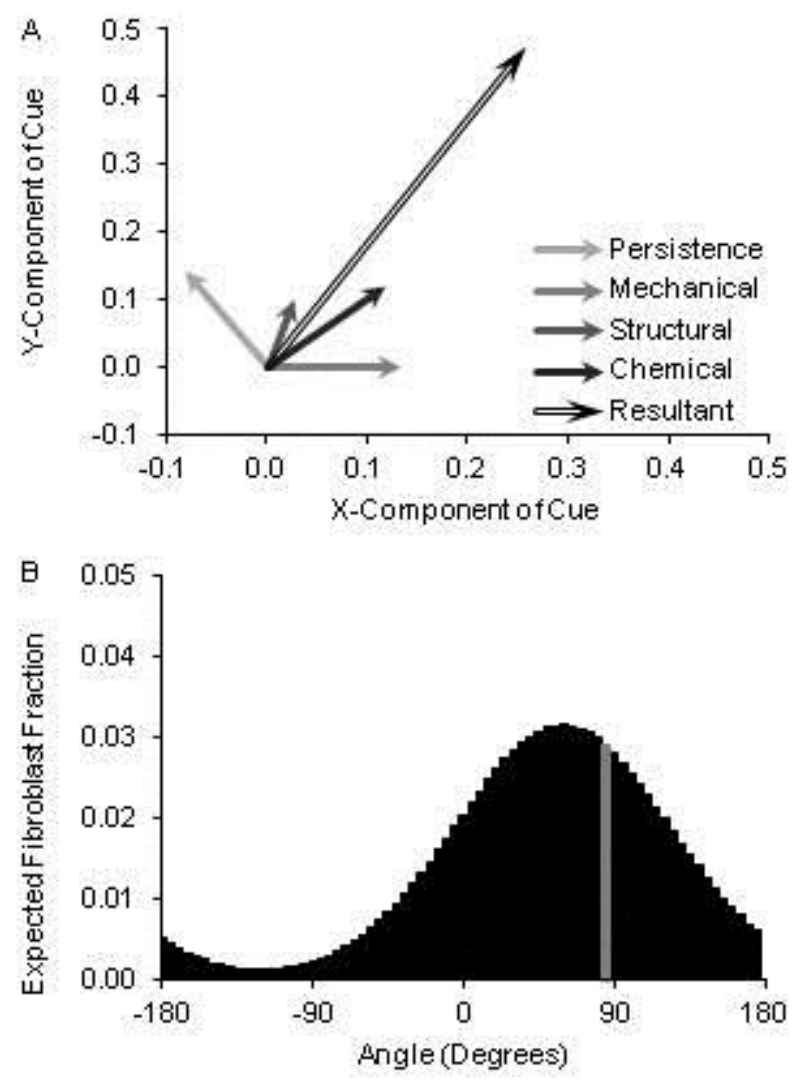

Figure 2.3. Method of determining fibroblast orientation. A. Each guidance cue was represented as a vector. The vectors were normalized, weighted, and averaged in order to compute a resultant vector representing the combined influence of all of the cues on the fibroblast orientation. B. The magnitude and orientation of the resultant vector defined the shape of a wrapped normal distribution. The new fibroblast orientation (indicated by the gray bar in the histogram) was chosen by taking a random sample from a set of angles representing this distribution. 


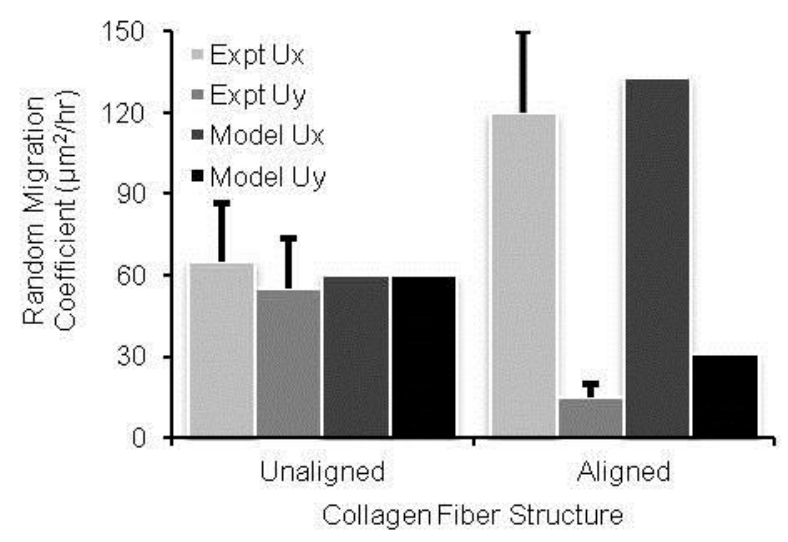

Figure 2.4. Fit of model to published fibroblast migration behavior in collagen gels. Dickinson et al. (1994) measured random migration coefficients for x-directed (Expt Ux) and y-directed (Expt Uy) motion of fibroblasts in collagen gels with unaligned or aligned collagen fibers. We obtained estimates of the model parameters $W_{p}, W_{s}$, and $\alpha$ by fitting simulated migration behavior (Model Ux, Model Uy) to the published data. 


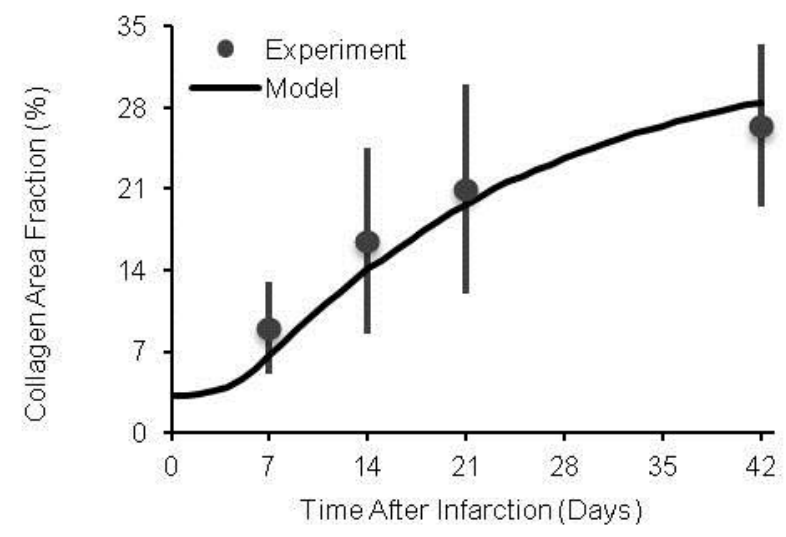

Figure 2.5. Fit of model to published infarct collagen accumulation time course. Fomovsky and Holmes (2010) measured collagen area fraction at several time points after creating ligation infarcts in rats (Experiment). We obtained estimates of the model parameters $k_{c f, g e n}$ and $k_{c f, d e g}$ by fitting a reaction ODE to the published data. Using the fitted parameter values, the agentbased model was able to reproduce the data (Model). 


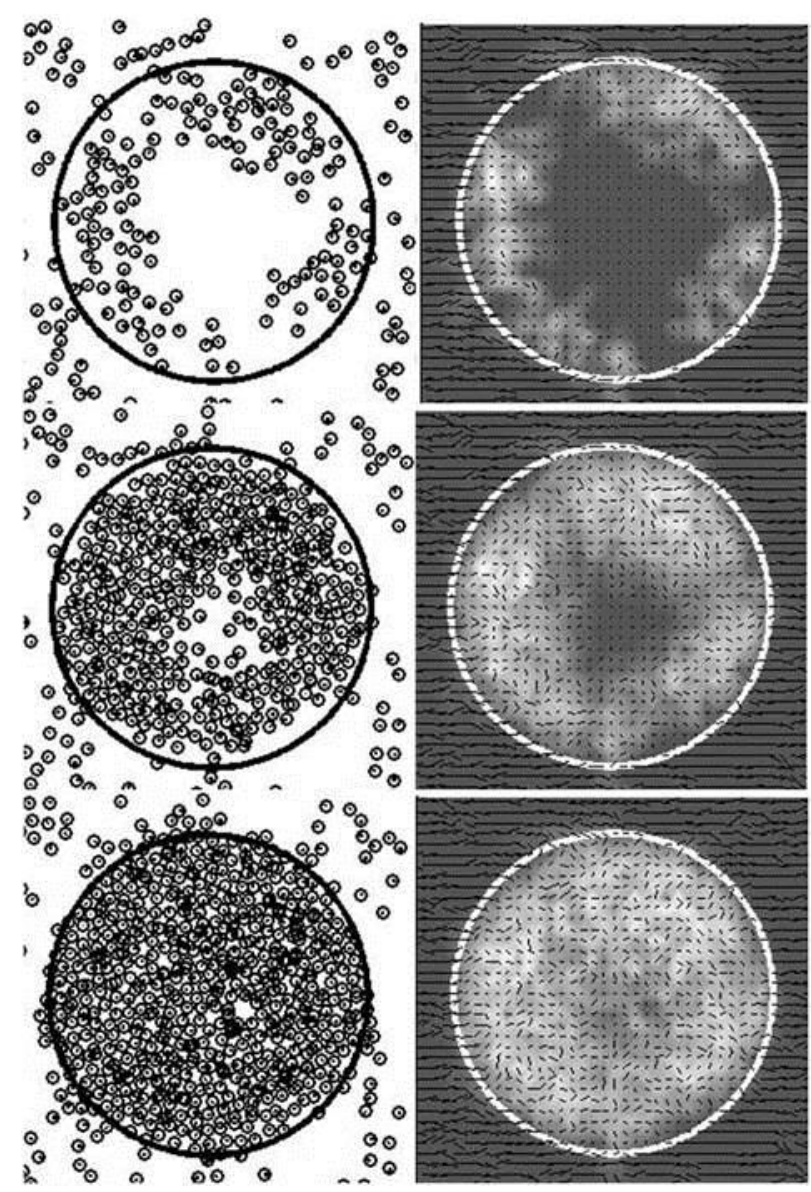

Figure 2.6. Time course of infarct healing. Chemokine generated within the infarct stimulated fibroblast migration and proliferation (left), and upregulated collagen deposition (right). The illustrated time points are 4 (top), 7 (middle), and 10 (bottom) days after infarction. 
A

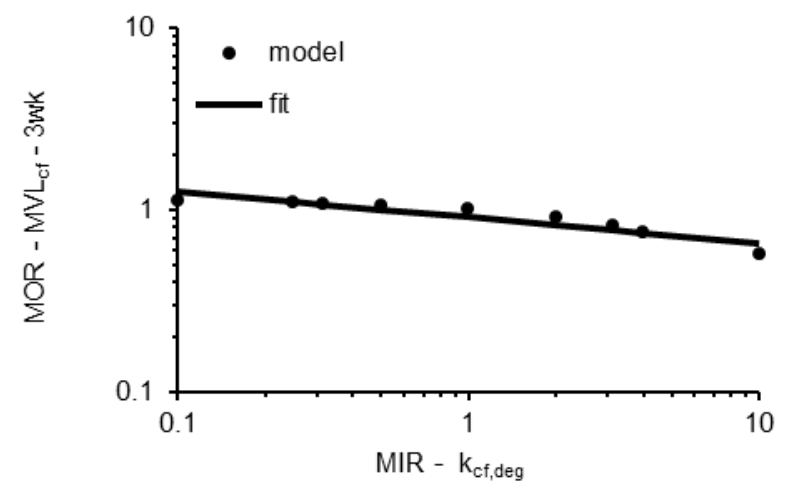

$\mathrm{C}$

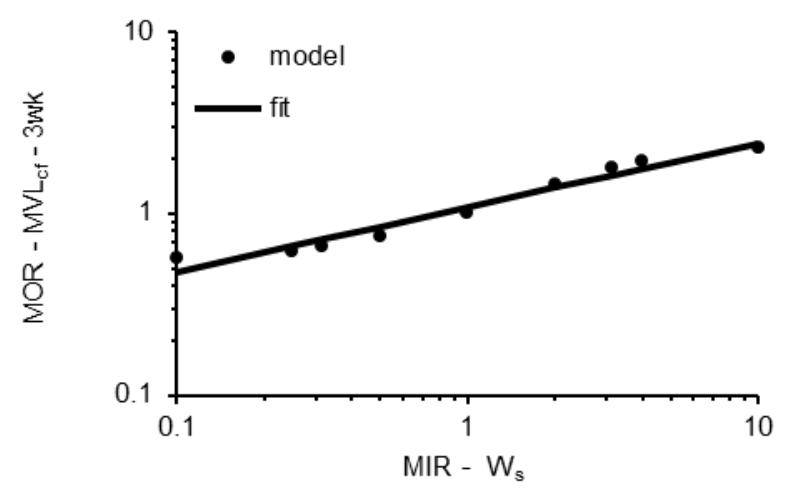

B

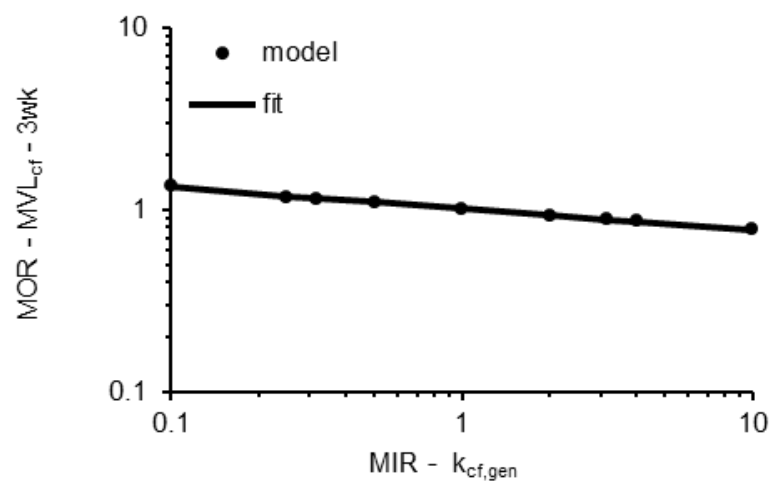

$\mathrm{D}$

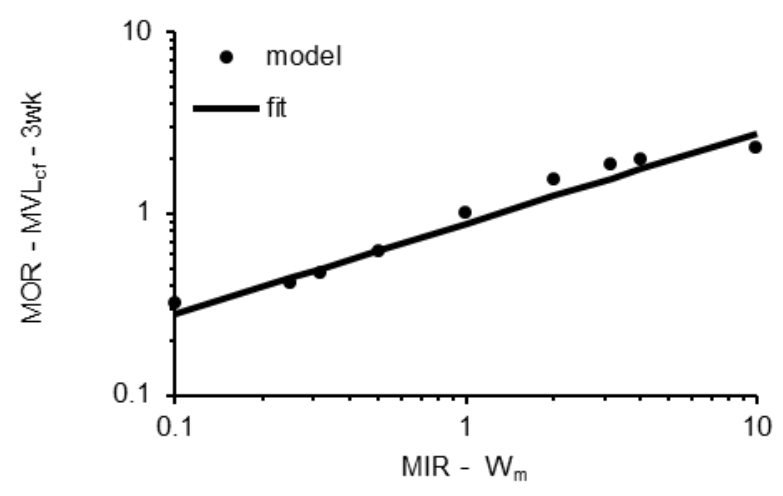

Figure 2.7. Example model input/output relationships. The output prediction is the strength of collagen fiber alignment in the infarct after 3 weeks of healing, quantified as mean vector length $\left(\mathrm{MVL}_{\mathrm{ff}}\right)$. The input parameters are the collagen fiber degradation rate coefficient $\left(\mathrm{k}_{\mathrm{cf}, \mathrm{deg}} ; \mathbf{A}\right)$, collagen fiber generation rate coefficient $\left(\mathrm{k}_{\mathrm{cf}, \mathrm{gen}} ; \mathbf{B}\right)$, structural guidance cue weight factor $\left(\mathrm{W}_{\mathrm{s}}\right.$, C), and mechanical guidance cue weight factor $\left(\mathrm{W}_{\mathrm{m}}, \mathbf{D}\right)$. Each input parameter value was varied from an order of magnitude below the baseline model value to an order of magnitude above the baseline model value. The sensitivity of the output prediction $\left(\mathrm{MVL}_{\mathrm{cf}}\right.$ at 3 weeks) to each input parameter was visualized by plotting the model output ratio (MOR) against the model input ratio (MIR), where the ratios were calculated as the perturbed model value divided by the baseline model value. For each relationship, sensitivity was quantified as the slope of the best-fit line to the log-transformed data. 

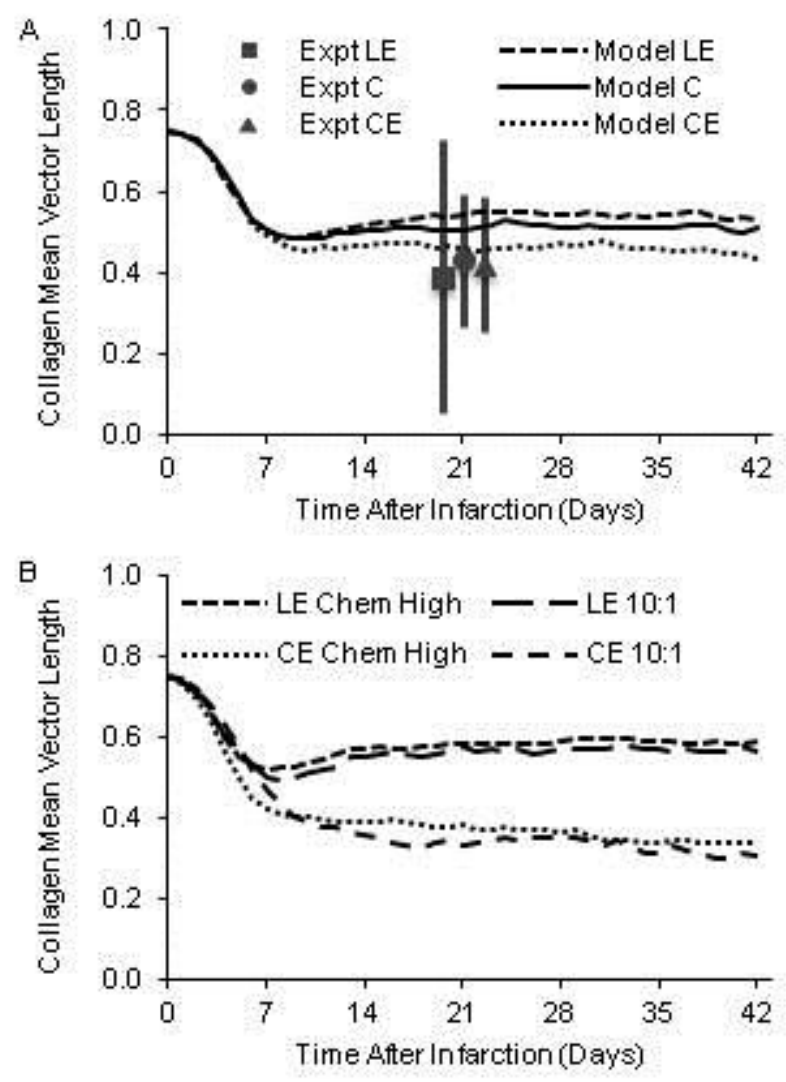

Figure 2.8. Simulations of circular and elliptical infarcts (2:1 aspect ratio) experiencing uniaxial strain with initial matrix alignment as found in native myocardium. A. The model predicted little difference in the strength of collagen alignment (quantified as the mean vector length of the average collagen fiber distribution) of a longitudinally oriented elliptical infarct (LE), circular infarct (C), and a circumferentially oriented elliptical infarct (CE). This was consistent with experimental data from our cryoinfarction study (Fomovsky et al., 2012b). B. By increasing the aspect ratio of the elliptical infarcts to 10:1 or increasing the chemical cue weight factor $W_{c}$ fourfold (Chem High), the model predicted a measurable effect of the orientation of the elliptical infarcts on the strength of collagen alignment. 

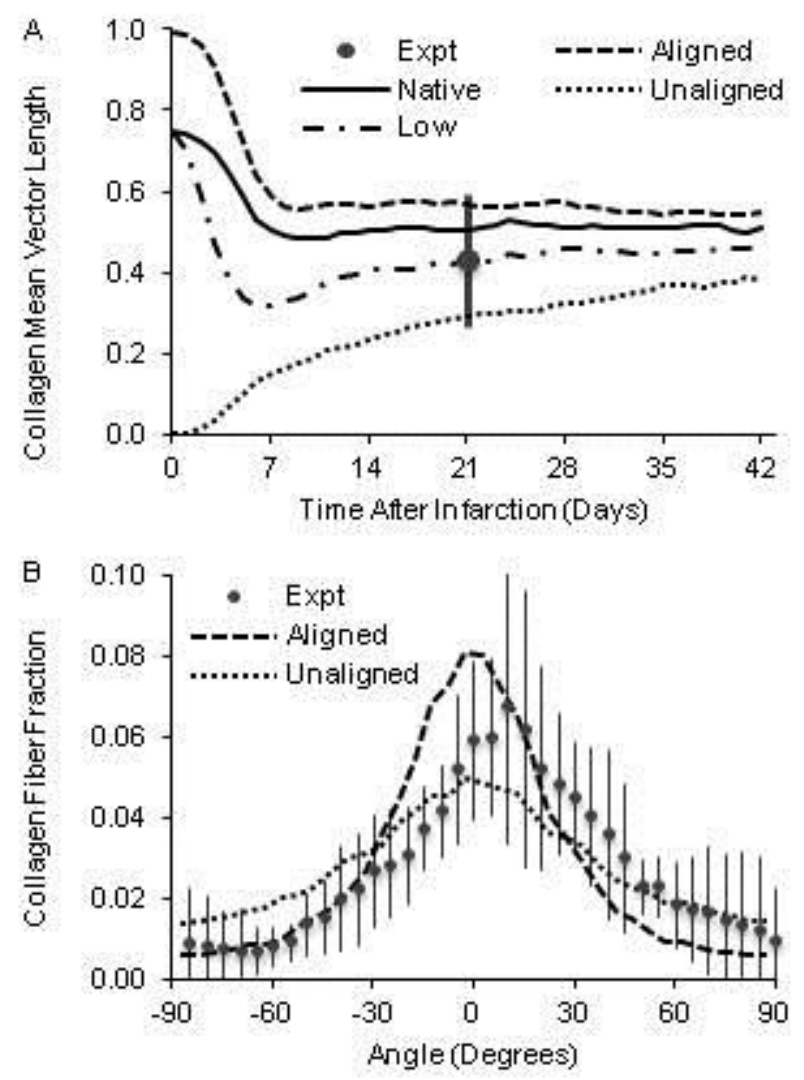

Figure 2.9. Simulations of circular infarcts experiencing uniaxial strain with different initial matrix alignment or density. A. The model predicted that infarcts with initial matrix structure that was either unaligned or perfectly aligned would have different collagen structures at early times, but gradually converge to the same degree of collagen alignment (Aligned vs Unaligned). Similarly, infarcts with initial matrix density that was either equal to or $1 / 10^{\text {th }}$ the density of normal myocardium had different collagen alignment in the first week and then gradually converged thereafter (Native vs Low). B. By 3 weeks, regardless of the strength of alignment of the initial matrix structure, the predicted collagen fiber orientation histograms fell within one standard deviation of the experimental mean for circular, mid-ventricular cryoinfarcts (Fomovsky et al., 2012b). 

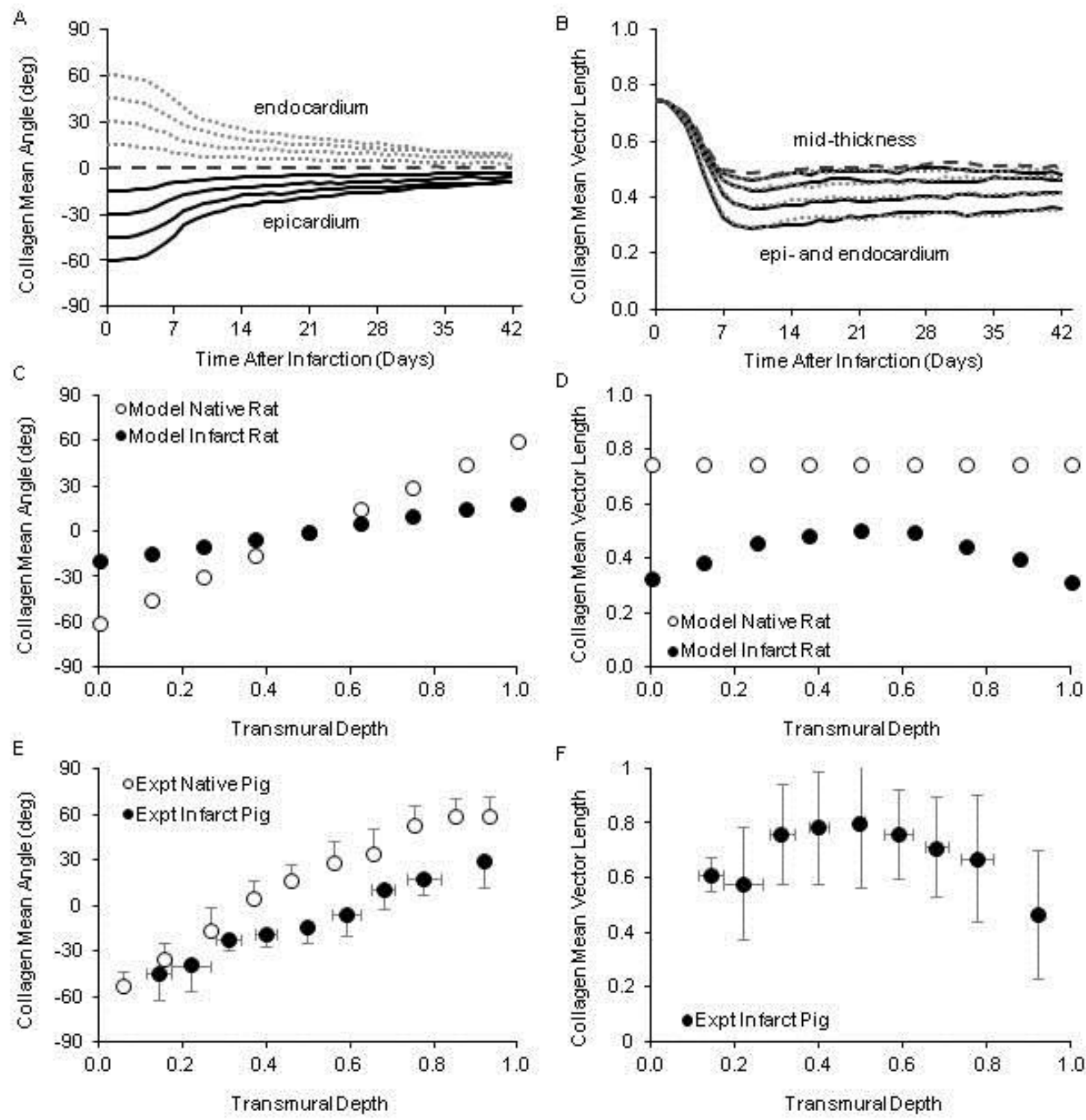

Figure 2.10. Simulations of circular infarcts experiencing uniaxial strain with mean fiber orientations of the initial matrix varying from -60 degrees at the epicardial surface ( $0 \%$ depth) to +60 degrees at the endocardial surface (100\% depth) to simulate a series of transmural layers in a healing infarct. A and B. The model predicted that mean collagen fiber angles gradually converged on the circumferential direction and suggested that mean vector length would converge more slowly than mean fiber angle. C and D. The model predicted a flatter transmural fiber angle profile in 3 week old infarcts than in healthy myocardium (Model Infarct Rat vs. Model Native Rat), predicted strongest alignment at mid-depth, where the direction of stretch and the initial matrix orientation were identical, and predicted weakest alignment at the surfaces, where initial matrix orientation differed most from the direction of stretch. E and F. Model predictions were similar to measurements before and 3 weeks after infarction in pigs (Expt Native Pig vs. Expt Infarct Pig) (Holmes, 1995; Holmes et al., 1997). 

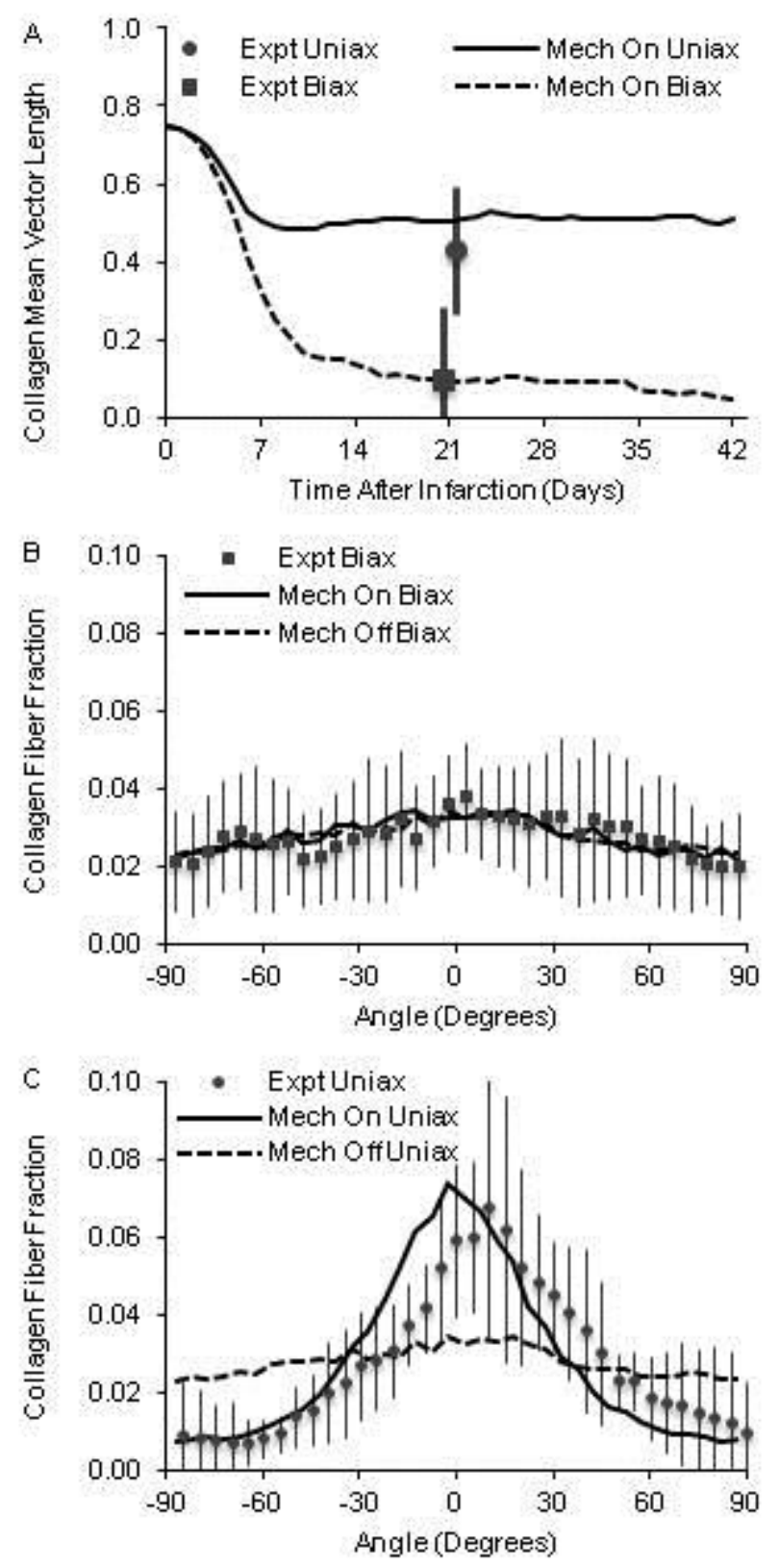

Figure 2.11. Simulations of circular infarcts with uniaxial or equibiaxial strains and with initial matrix alignment as found in native myocardium. A. The model predicted that long term alignment of collagen in a healing infarct required a uniaxial mechanical cue, which was consistent with experimental data from our cryoinfarction study (Expt Uniax vs Expt Biax). B and C. At 3 weeks, with mechanosensing turned on (Mech On), the model predicted collagen fiber orientation histograms similar to those measured in cryoinfarcts experiencing biaxial or uniaxial strain. With mechanosensing turned off (Mech Off), the aligned collagen structure for the case of uniaxial strain could not be predicted. 
A

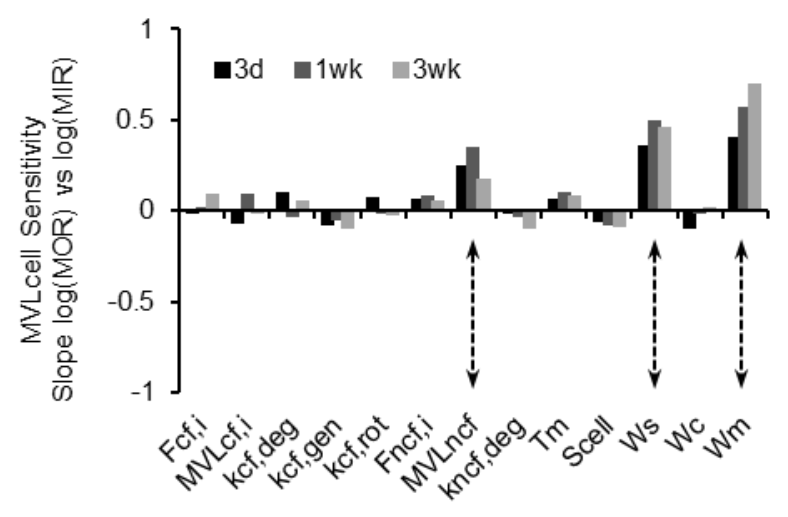

$\mathrm{C}$

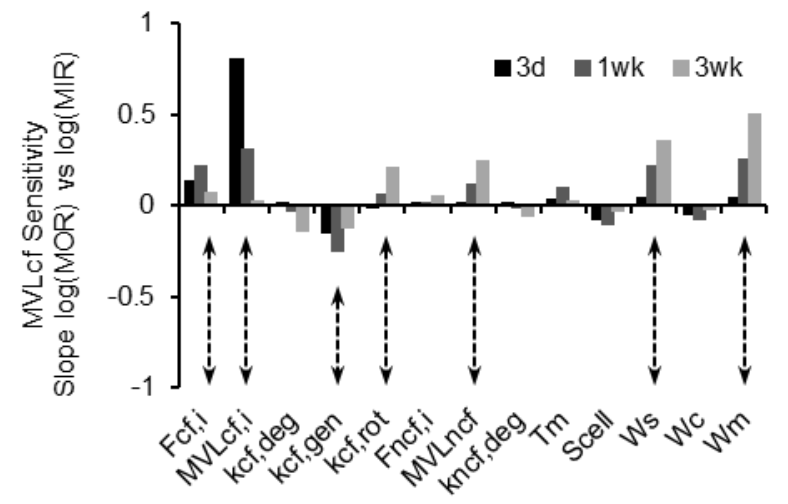

B

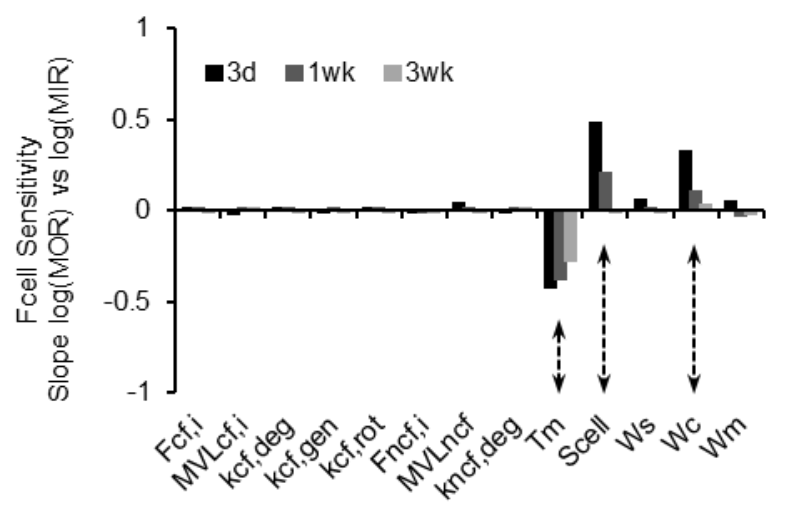

$\mathrm{D}$

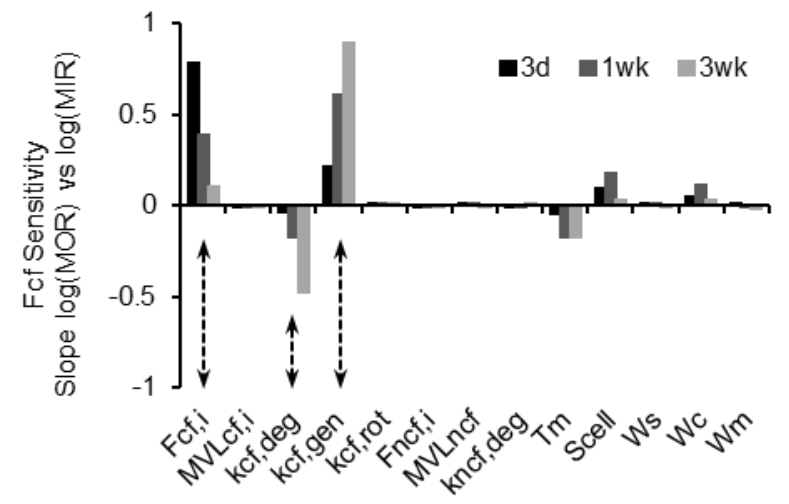

Figure 2.12. Sensitivities of model input/output relationships. A. Sensitivity of the strength of cell alignment within the infarct to each input parameter, measured after 3 days, 1 week, and 3 weeks of healing. B. Sensitivity of the cell concentration. C. Sensitivity of the strength of collagen fiber alignment. D. Sensitivity of the collagen matrix density. Strength of alignment was quantified as the mean vector length of the set of cell orientations or fiber orientations within the infarct. Cell concentration and collagen matrix density were quantified as number of cells or fibers within the infarct and then converted to area fraction. Sensitivities were measured as the slope of the $\log _{10}$ output ratio plotted against the $\log _{10}$ input ratio. Sensitivities of 1 or -1 indicated proportional or inversely proportional relationships, respectively. Relationships with sensitivity magnitudes less than or equal to 0.194 were considered insensitive. 
Table 2.1. List of model input/output relationships that satisfied the sensitivity threshold

\begin{tabular}{|c|c|c|c|c|c|c|c|c|}
\hline Output & Time & \multicolumn{7}{|c|}{ Inputs Above Sensitivity Threshold } \\
\hline $\mathrm{MVL}_{\text {cell }}$ & $\begin{array}{c}3 \mathrm{~d} \\
1 \mathrm{wk} \\
3 \mathrm{wk}\end{array}$ & $\begin{array}{l}\text { MVLncf } \\
\text { MVL } L_{n f}\end{array}$ & $\begin{array}{l}W_{s} \\
W_{s} \\
W_{s}\end{array}$ & $\begin{array}{l}\mathrm{W}_{\mathrm{m}} \\
\mathrm{W}_{\mathrm{m}} \\
\mathrm{W}_{\mathrm{m}}\end{array}$ & & & & \\
\hline $\mathrm{F}_{\text {cell }}$ & $\begin{array}{c}3 \mathrm{~d} \\
1 \mathrm{wk} \\
3 \mathrm{wk}\end{array}$ & $\begin{array}{l}\mathrm{T}_{\mathrm{m}} \\
\mathrm{T}_{\mathrm{m}} \\
\mathrm{T}_{\mathrm{m}}\end{array}$ & $\begin{array}{l}\mathrm{S}_{\text {cell }} \\
\mathrm{S}_{\text {cell }}\end{array}$ & $\mathrm{W}_{\mathrm{c}}$ & & & & \\
\hline $\mathrm{MVL}_{\mathrm{cf}}$ & $\begin{array}{c}3 \mathrm{~d} \\
1 \mathrm{wk} \\
3 \mathrm{wk}\end{array}$ & $\mathrm{F}_{\mathrm{cf}, \mathrm{i}}$ & $\begin{array}{l}\text { MVL }_{c f, i} \\
\text { MVL }_{c f, i}\end{array}$ & $\mathrm{k}_{\mathrm{cf}, \mathrm{gen}}$ & $\mathrm{k}_{\mathrm{cf}, \mathrm{rot}}$ & MVLncf & $\begin{array}{l}W_{s} \\
W_{s}\end{array}$ & $\begin{array}{l}\mathrm{W}_{\mathrm{m}} \\
\mathrm{W}_{\mathrm{m}}\end{array}$ \\
\hline $\mathrm{F}_{\mathrm{cf}}$ & $\begin{array}{c}3 \mathrm{~d} \\
1 \mathrm{wk} \\
3 \mathrm{wk}\end{array}$ & $\begin{array}{l}F_{\mathrm{cff}, \mathrm{i}} \\
\mathrm{F}_{\mathrm{cf}, \mathrm{i}}\end{array}$ & $\mathrm{k}_{\mathrm{cf}, \mathrm{deg}}$ & $\begin{array}{l}\mathrm{k}_{\mathrm{cf}, \text { gen }} \\
\mathrm{k}_{\mathrm{cf} \text {,gen }} \\
\mathrm{k}_{\mathrm{cf}, \text { gen }}\end{array}$ & & & & \\
\hline
\end{tabular}

$\mathrm{MVL}_{\text {cell }}$ - strength of alignment of cells (mean vector length), $\mathrm{F}_{\text {cell }}$ - concentration of cells (area fraction), $\mathrm{MVL}_{\mathrm{cf}}$ - strength of alignment of collagen matrix, $\mathrm{F}_{\mathrm{cf}}$ - density of collagen matrix, $\mathrm{MVL}_{\mathrm{ncf}}$ - strength of alignment of non-collagen matrix, $\mathrm{W}_{\mathrm{s}}$ - structural cue weight factor, $\mathrm{W}_{\mathrm{m}}$ - mechanical cue weight factor, $\mathrm{W}_{\mathrm{c}}$ - chemical cue weight factor, $\mathrm{T}_{\mathrm{m}}$ - cell cycle duration (time to mitosis), $S_{\text {cell }}$ - cell migration speed, $F_{c f, i}$ - initial density of collagen matrix, $\mathrm{MVL}_{\mathrm{cf}, \mathrm{i}}$ - initial strength of alignment of collagen matrix, $\mathrm{k}_{\mathrm{cf}, \mathrm{gen}}-$ collagen fiber generation rate coefficient, $\mathrm{k}_{\mathrm{cf}, \mathrm{rot}}-$ collagen fiber rotation rate coefficient, $\mathrm{k}_{\mathrm{cf}, \mathrm{deg}}-$ collagen fiber degradation rate coefficient 
Table 2.2. Model input ratio (MIR) lower and upper bounds for each of the input parameters to which the model was sensitive. These bounds define the range over which variation of any one of these inputs would not appreciably change any of the model outputs of interest. The "Threshold Output" column lists the model predictions that were most sensitive to the input parameters.

\begin{tabular}{|c|c|c|c|}
\hline Input Parameter & $\underline{\mathrm{MIR}}_{\mathrm{LB}}$ & $\underline{\mathrm{MIR}_{U B}}$ & Threshold Output \\
\hline MVLncf & 0.51 & 1.3 & $\mathrm{MVL}_{\text {cell, } 3 \mathrm{~d}}$ \\
\hline $\mathrm{MVL}_{\mathrm{cf}, \mathrm{i}}$ & 0.51 & 1.3 & $\mathrm{MVL}_{\mathrm{cf}, 3 \mathrm{~d}}$ \\
\hline $\mathrm{F}_{\mathrm{cf,i}}$ & 0.42 & 1.3 & $\mathrm{~F}_{\mathrm{cf}, 3 \mathrm{~d}}$ \\
\hline $\mathrm{T}_{\mathrm{m}}$ & 0.44 & 2.6 & $\mathrm{~F}_{\text {cell, 3d }}$ \\
\hline$S_{\text {cell }}$ & 0.46 & 3.0 & $\mathrm{~F}_{\text {cell, 3d }}$ \\
\hline $\mathrm{W}_{\mathrm{c}}$ & 0.23 & 3.8 & $\mathrm{~F}_{\text {cell, 3d }}$ \\
\hline $\mathrm{W}_{\mathrm{m}}$ & 0.39 & 1.4 & $\mathrm{MVL}_{\text {cell, }}$ 3wk \\
\hline $\mathrm{W}_{\mathrm{s}}$ & 0.27 & 1.6 & $\mathrm{MVL}_{\text {cell, }}$ 3wk \\
\hline $\mathrm{k}_{\mathrm{cf} \text {,gen }}$ & 0.50 & 1.4 & $\mathrm{~F}_{\mathrm{cf}, 3 \mathrm{wk}}$ \\
\hline $\mathrm{k}_{\mathrm{cf}, \mathrm{deg}}$ & 0.21 & 2.3 & $\mathrm{~F}_{\mathrm{cf}, 3 \mathrm{wk}}$ \\
\hline $\mathrm{k}_{\mathrm{cf}, \mathrm{rot}}$ & 0.13 & 9.7 & $\mathrm{MVL}_{c f, 3 w k}$ \\
\hline mean & 0.37 & 2.7 & \\
\hline
\end{tabular}




\section{APPENDIX 2.A - Calculation of Guidance Cue Vectors}

In order to compute the vector for the chemical cue, the chemokine concentration at the cell boundary was multiplied by the unit outward normal vector, and this scaled vector was averaged over the cell boundary. The result was a vector pointing in the direction of greatest chemokine concentration at the cell boundary with magnitude determined by the steepness of the local chemokine gradient.

$\vec{v}_{c}=\frac{\oint C_{c}\left(R_{c e l l}, \theta\right) \vec{n} d s}{\oint d s}=\frac{\int_{-\pi}^{\pi} C_{c}\left(R_{c e l l}, \theta\right) \vec{n} R_{c e l l} d \theta}{\int_{-\pi}^{\pi} R_{c e l l} d \theta}=\frac{1}{2 \pi} \int_{-\pi}^{\pi} C_{C}\left(R_{c e l l}, \theta\right) \vec{n} d \theta$

$\vec{v}_{c}$ - chemical guidance cue vector, $C_{c}$ - chemokine concentration, $\vec{n}$ - unit outward normal vector from cell surface, $R_{\text {cell }}$ - cell radius

In order to compute the vector for the mechanical cue, the normal strain at the cell boundary was multiplied by the unit outward normal vector with doubled angle, and this scaled vector was averaged over the cell boundary. The $2 \theta$ transform was performed because tension on the cell is an axial cue, rather than a directional cue, so anti-parallel strains should sum instead of canceling. The result was transformed from $2 \theta$ space back to $\theta$ space by halving the angle of the computed vector. The result was a vector pointing in the direction of greatest tensile strain across the cell with magnitude determined by the degree of anisotropy of the local strain field. 
$\varepsilon_{n}=\vec{n}^{T}(\overline{\bar{\varepsilon}} \vec{n})$

$\vec{v}_{m, 2 \theta}=\frac{\int_{-\pi}^{\pi} \varepsilon_{n}\left(R_{\text {cell }}, \theta\right) \vec{n}(2 \theta) R_{\text {cell }} d \theta}{\int_{-\pi}^{\pi} R_{\text {cell }} d \theta}=\frac{1}{2 \pi} \int_{-\pi}^{\pi} \varepsilon_{n}\left(R_{\text {cell }}, \theta\right) \vec{n}(2 \theta) d \theta$

$\theta_{m}=\frac{1}{2} \angle \vec{v}_{m, 2 \theta}$

$\vec{v}_{m}=\left\|\vec{v}_{m, 2 \theta}\right\| \vec{u}\left(\theta_{m}\right)$

$\varepsilon_{n}$ - strain normal to cell surface, $\vec{n}$ - unit outward normal vector from cell surface, $\overline{\bar{\varepsilon}}$ - strain tensor, $\vec{v}_{m, 2 \theta}$ - mechanical guidance cue vector in $2 \theta$ space, $R_{\text {cell }}$ - cell radius, $\theta_{m}$ - mechanical guidance cue direction in $\theta$ space, $\vec{v}_{m}$ - mechanical guidance cue vector in $\theta$ space.

In order to compute the vector for the structural cue, the mean vector length and mean angle of the total population of collagen and non-collagen fibers located within the cell boundary were calculated. Again, the $2 \theta$ transform was performed because fiber direction is an axial cue, rather than a directional cue. The result was a vector pointing in the mean direction of the local matrix fibers with magnitude determined by the degree of co-alignment of the fibers.

$\vec{v}_{s, 2 \theta}=\frac{\int_{-\pi}^{\pi} N_{f}(\theta) \vec{u}(2 \theta) d \theta}{\int_{-\pi}^{\pi} N_{f}(\theta) d \theta}$

$\theta_{S}=\frac{1}{2} \square \angle \vec{v}_{S, 2 \theta}$

$\vec{v}_{s}=\left\|\vec{v}_{s, 2 \theta}\right\| \vec{u}\left(\theta_{s}\right)$

$\vec{v}_{s, 2 \theta}$ - structural guidance cue vector in $2 \theta$ space, $N_{f}$ - number of fibers located within the cell boundary, $\vec{u}$ - unit vector, $\theta_{s}$ - structural guidance cue direction in $\theta$ space, $\vec{v}_{s}$ - structural guidance cue vector in $\theta$ space. 
In order to compute the vector for the persistence cue, the unit vector pointing in the direction of the cell was multiplied by the cell speed.

$\vec{v}_{p}=S_{\text {cell }} \vec{u}\left(\theta_{\text {cell }}\right)$

$\vec{v}_{p}$ - persistence cue vector, $S_{c e l l}$ - current cell migration speed, $\vec{u}$ - unit vector, $\theta_{\text {cell }}-$ current cell orientation 


\section{APPENDIX 2.B - List of Model Parameters}

\section{Descriptive Parameters}

\section{Infarct Dimensions}

\begin{tabular}{|llllll|}
\hline Geometric Scaling Factor $^{\mathrm{a}}$ & $\lambda_{\mathrm{g}}$ & 0.040 & & \\
Area $^{\mathrm{b}}$ & $\mathrm{A}_{\mathrm{i}}$ & $25 \lambda_{\mathrm{g}}{ }^{2}$ & $\mathrm{~mm}^{2}$ & & \\
& & & & & \\
& & Circle & Ellipse & \\
Major Radius & $\mathrm{R}_{\text {maj }}$ & $2830 \lambda_{\mathrm{g}}$ & $4000 \lambda_{\mathrm{g}}$ & $\mu \mathrm{m}$ & (Fomovsky et al., 2012b) \\
Minor Radius $^{\mathrm{b}}$ & $\mathrm{R}_{\text {min }}$ & $2830 \lambda_{\mathrm{g}}$ & $2000 \lambda_{\mathrm{g}}$ & $\mu \mathrm{m}$ & (Fomovsky et al., 2012b) \\
\hline
\end{tabular}

a: Geometric scaling factor explained in the methods section titled "Scaling".

b: Dimensions calculated for circle and 2:1 ellipse based on average cryoinfarct area reported by Fomovsky et al. (2012b).

\section{Fibroblast Size and Initial Concentration (normal myocardium)}

\begin{tabular}{|c|c|c|c|c|}
\hline Radius $^{c}$ & $\mathrm{R}_{\text {cell }}$ & 5 & $\mu \mathrm{m}$ & (Camelliti et al., 2005) \\
\hline Area Fraction & $F_{f}$ & 0.25 & & (Vliegen et al., 1991) \\
\hline
\end{tabular}

c: Radius of simplified, disk-shaped fibroblast calculated from fibroblast area estimated from images in Camelliti et al. (2005).

Initial Fiber Structure (normal myocardium)

\begin{tabular}{|c|c|c|c|c|c|}
\hline Collagen Fiber Area Fraction & $\mathrm{F}_{\mathrm{cf}}$ & 0.03 & & & (Weber, 1989) \\
\hline Non-Collagen Fiber Area Fraction & $\mathrm{F}_{\mathrm{ncf}}$ & 0.72 & & & (Vliegen et al., 1991) \\
\hline Mean Vector Length & MVL & 0.75 & & & (unpublished observation) \\
\hline Mean Angle & MA & $\begin{array}{l}\text { epicardium } \\
-60^{\circ}\end{array}$ & mid-depth & $\begin{array}{l}\text { endocardium } \\
60^{\circ}\end{array}$ & (Omens et al 1993) \\
\hline
\end{tabular}

\section{Strain Field}

\begin{tabular}{|c|c|c|c|c|c|}
\hline \multirow[t]{2}{*}{ Border Zone Strain Transition Width ${ }^{d}$} & $\sigma_{\mathrm{b}}$ & \multicolumn{3}{|c|}{$0.08 \sqrt{ }\left(4 \mathrm{R}_{\mathrm{maj}} \mathrm{R}_{\mathrm{min}}\right)$} & \multirow[t]{2}{*}{ (Gallagher et al., 1986) } \\
\hline & & Normal & Apical Infarct & Anterior Infarct & \\
\hline Circumferential Strain & $\varepsilon_{\mathrm{c}}$ & -0.05 & 0.05 & 0.05 & (Fomovsky et al., 2012b) \\
\hline Longitudinal Strain & $\varepsilon_{1}$ & -0.05 & 0.05 & 0 & (Fomovsky et al., 2012b) \\
\hline
\end{tabular}

$\mathrm{d}$ : The width of the strain transition was about $8 \%$ of the width of the infarcts studied by Gallagher et al. (1986). We generalized the infarct width to be the average infarct diameter $=\sqrt{ }\left(4 R_{\mathrm{maj}} R_{\mathrm{min}}\right)$.

\section{Chemokine Concentration Field}

\begin{tabular}{|lllll|}
\hline Diffusion Coefficient & $\mathrm{D}_{\mathrm{c}}$ & $100 \mathrm{\lambda}_{\mathrm{g}}{ }^{2}$ & $\mathrm{\mu m}^{2} / \mathrm{s}$ & (Safford et al., 1978) \\
Degradation Rate Coefficient & $\mathrm{k}_{\mathrm{c}, \mathrm{deg}}$ & 0.001 & $\mathrm{~s}^{-1}$ & (Bailón-Plaza \& Van der Meulen, 2001) \\
Generation Rate Coefficient $^{\mathrm{e}}$ & $\mathrm{k}_{\mathrm{c}, \mathrm{gen}}$ & 0.01 & $\mathrm{nM} / \mathrm{s}$ & \\
\hline
\end{tabular}

e: The generation rate coefficient is an arbitrary value. It determines the magnitude but not the shape of the chemokine concentration profile. Its value is not important since calculations involving the chemokine concentration are normalized. 


\section{Behavioral Parameters}

\section{Fiber Remodeling}

\begin{tabular}{|c|c|c|c|c|c|}
\hline Collagen Degradation Rate Coefficient ${ }^{\dagger}$ & $\mathrm{K}_{\mathrm{cf}, \mathrm{deg}}^{\prime}$ & $\begin{array}{l}\text { Normal } \\
2.5 \mathrm{E}-4\end{array}$ & $\begin{array}{l}\text { Infarct } \\
2.5 E-3\end{array}$ & $\mathrm{hr}^{-1}$ & (Fomovsky \& Holmes, 2010) \\
\hline Collagen Generation Rate Coefficient ${ }^{\dagger}$ & $\mathrm{k}_{\mathrm{cf}, \mathrm{gen}}^{\prime}$ & $7.5 \mathrm{E}-4$ & 7.3E-2 & $\%$ area $/ \mathrm{hr}$ & (Fomovsky \& Holmes, 2010) \\
\hline Collagen Fiber Rotation Rate Coefficient ${ }^{g}$ & $\mathrm{k}_{\mathrm{cf}, \mathrm{rot}}$ & 5 & 5 & $\mathrm{deg} / \mathrm{hr}$ & \\
\hline Non-Collagen Degradation Rate Coefficient ${ }^{\mathrm{h}}$ & $\mathrm{K}_{\text {nct,deg }}^{\prime}$ & & $2.5 \mathrm{E}-3$ & $\mathrm{hr}^{-1}$ & \\
\hline
\end{tabular}

$\mathrm{f}$ : Calculation of the collagen degradation and generation rates in the infarct is explained in the methods section titled "Parameter Specification". The degradation rate in normal myocardium was assumed to be an order of magnitude slower than in the infarct.

The collagen generation rate in normal myocardium was calculated to ensure a steady collagen area fraction of 0.03 .

g: Collagen rotation rate coefficient approximated from observations of fibroblast-mediated remodeling of collagen gels.

$\mathrm{h}$ : Non-collagen degradation rate assumed to be the same as the collagen degradation rate.

\section{Fibroblast Dynamics}

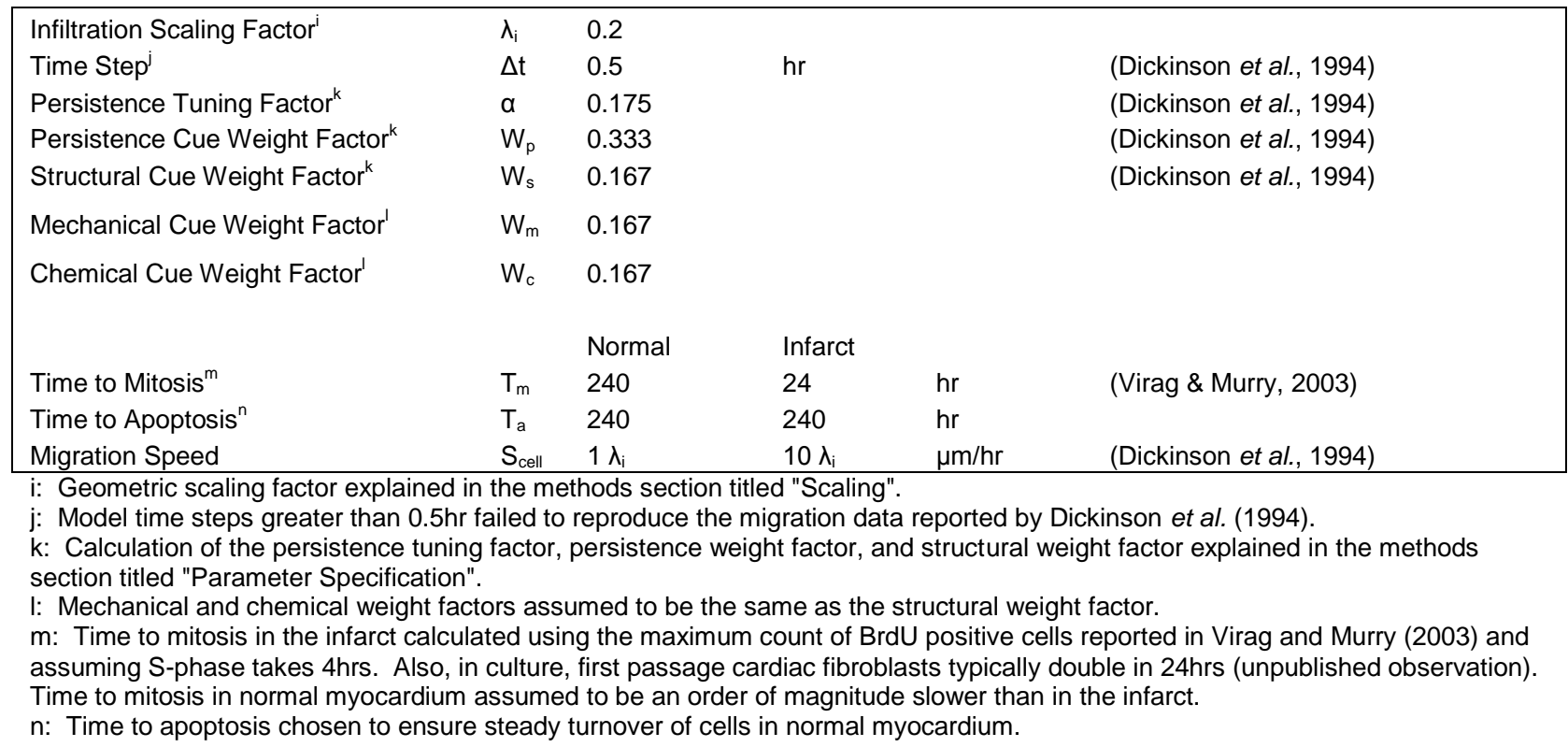




\subsection{REFERENCES}

Bailón-Plaza A \& Van der Meulen MCH (2001). A mathematical framework to study the effects of growth factor influences on fracture healing. Journal of Theoretical Biology 212, 191209.

Camelliti P, Borg TK \& Kohl P (2005). Structural and functional characterisation of cardiac fibroblasts. Cardiovascular Research 65, 40-51.

Canty EG, Lu Y, Meadows RS, Shaw MK, Holmes DF \& Kadler KE (2004). Coalignment of plasma membrane channels and protrusions (fibripositors) specifies the parallelism of tendon. Journal of Cell Biology 165, 553-563.

Chavali AK, Gianchandani EP, Tung KS, Lawrence MB, Peirce SM \& Papin JA (2008). Characterizing emergent properties of immunological systems with multi-cellular rulebased computational modeling. Trends in Immunology 29, 589-599.

Christman KL, Fok HH, Sievers RE, Fang Q \& Lee RJ (2004). Fibrin glue alone and skeletal myoblasts in a fibrin scaffold preserve cardiac function after myocardial infarction. Tissue Engineering 10, 403-409.

Chung E, Dan D, Solomon S, Bank A, Pastore J, Iyer A, Berger R, Franklin J, Jones G, Machado C \& Stolen C (2010). Effect of peri-infarct pacing early after myocardial infarction: results of the prevention of myocardial enlargement and dilatation post myocardial infarction study. Circulation Heart Failure 3, 650-658.

Dickinson RB, Guido S \& Tranquillo RT (1994). Biased cell migration of fibroblasts exhibiting contact guidance in oriented collagen gels. Annals of Biomedical Engineering 22, 342-356.

Fomovsky GM, Clark SA, Parker KM, Ailawadi G \& Holmes JW (2012a). Anisotropic reinforcement of acute anteroapical infarcts improves pump function. Circulation Heart Failure 5, 515-522.

Fomovsky GM \& Holmes JW (2010). Evolution of scar structure, mechanics, and ventricular function after myocardial infarction in the rat. American Journal of Physiology: Heart and Circulatory Physiology 298, H221-H228.

Fomovsky GM, Macadangdang JR, Ailawadi G \& Holmes JW (2011). Model-based design of mechanical therapies for myocardial infarction. Journal of Cardiovascular Translational Research 4, 82-91.

Fomovsky GM, Rouillard AD \& Holmes JW (2012b). Regional mechanics determine collagen fiber structure in healing myocardial infarcts. Journal of Molecular and Cellular Cardiology 52, 1083-1090. 
Gallagher KP, Gerren RA, Stirling MC, Choy M, Dysko RC, McManimon SP \& Dunham WR (1986). The distribution of functional impairment across the lateral border of acutely ischemic myocardium. Circulation Research 58, 570-583.

Groh A \& Wagner M (2011). Biased three-dimensional cell migration and collagen matrix modification. Mathematical Biosciences 231, 105-119.

Gupta KB, Ratcliffe MB, Fallert MA, Edmunds LH \& Bogen DK (1994). Changes in passive mechanical stiffness of myocardial tissue with aneurysm formation. Circulation 89, 23152326.

Holmes JW (1995). Remodeling, Deformation, and Tissue Structure in Healing Myocardial Infarcts (thesis). University of California San Diego.

Holmes JW, Nuñez JA \& Covell JW (1997). Functional implications of myocardial scar structure. American Journal of Physiology: Heart and Circulatory Physiology 272, H2123H2130.

Holmes JW, Yamashita H, Waldman LK \& Covell JW (1994). Scar remodeling and transmural deformation after infarction in the pig. Circulation 90, 411-420.

Hudson MP, Armstrong PW, Ruzyllo W, Brum J, Cusmano L, Krzeski P, Lyon R, Quinones M, Theroux P, Sydlowski D, Kim HE, Garcia MJ, Jaber WA \& Weaver WD (2006). Effects of selective matrix metalloproteinase inhibitor (PG-116800) to prevent ventricular remodeling after myocardial infarction: results of the PREMIER (Prevention of Myocardial Infarction Early Remodeling) trial. Journal of the American College of Cardiology 48, 15-20.

Javierre E, Moreo P, Doblaré M \& García-Aznar JM (2009). Numerical modeling of a mechanochemical theory for wound contraction analysis. International Journal of Solids and Structures 46, 3597-3606.

Jin Y, Han H, Berger J, Dai Q \& Lindsey ML (2011). Combining experimental and mathematical modeling to reveal mechanisms of macrophage-dependent left ventricular remodeling. BMC Systems Biology 5, 1-14.

Kadler KE, Hill A \& Canty-Laird EG (2008). Collagen fibrillogenesis: fibronectin, integrins, and minor collagens as organizers and nucleators. Current Opinion in Cell Biology 20, 495-501.

Kelley ST, Malekan R, Gorman JH, Jackson BM, Gorman RC, Suzuki Y, Plappert T, Bogen DK, Sutton MG \& Edmunds LH (1999). Restraining infarct expansion preserves left ventricular geometry and function after acute anteroapical infarction. Circulation 99, 135-142.

Knapp DM, Helou EF \& Tranquillo RT (1999). A fibrin or collagen gel assay for tissue cell chemotaxis: assessment of fibroblast chemotaxis to GRGDSP. Experimental Cell Research 247, 543-553. 
Laeremans H, Hackeng TM, Van Zandvoort MAMJ, Thijssen VLJL, Janssen BJA, Ottenheijm HCJ, Smits JFM \& Blankesteijn WM (2011). Blocking of frizzled signaling with a homologous peptide fragment of $\mathrm{Wnt} 3 \mathrm{a} / \mathrm{Wnt} 5 \mathrm{a}$ reduces infarct expansion and prevents the development of heart failure after myocardial infarction. Circulation 124, 1626-1635.

Lee EJ, Holmes JW \& Costa KD (2008). Remodeling of engineered tissue anisotropy in response to altered loading conditions. Annals of Biomedical Engineering 36, 1322-1334.

Mazhari R, Omens JH, Covell JW \& McCulloch AD (2000). Structural basis of regional dysfunction in acutely ischemic myocardium. Cardiovascular Research 47, 284-293.

McDougall S, Dallon J, Sherratt J \& Maini P (2006). Fibroblast migration and collagen deposition during dermal wound healing: mathematical modelling and clinical implications. Philosophical Transactions of the Royal Society A: Mathematical, Physical and Engineering Sciences 364, 1385-1405.

Melvin AT, Welf ES, Wang Y, Irvine DJ \& Haugh JM (2011). In chemotaxing fibroblasts, both high-fidelity and weakly biased cell movements track the localization of PI3K signaling. Biophysical Journal 100, 1893-1901.

Mi Q, Rivière B, Clermont G, Steed DL \& Vodovotz Y (2007). Agent-based model of inflammation and wound healing: insights into diabetic foot ulcer pathology and the role of transforming growth factor-beta1. Wound Repair and Regeneration 15, 671-682.

Neidlinger-Wilke C, Grood ES, Wang J H-C, Brand RA \& Claes L (2001). Cell alignment is induced by cyclic changes in cell length: studies of cells grown in cyclically stretched substrates. Journal of Orthopaedic Research 19, 286-293.

Ohsumi TK, Flaherty JE, Evans MC \& Barocas VH (2008). Three-dimensional simulation of anisotropic cell-driven collagen gel compaction. Biomechanics and Modeling in Mechanobiology 7, 53-62.

Omens JH, MacKenna DA \& McCulloch AD (1993). Measurement of strain and analysis of stress in resting rat left ventricular myocardium. Journal of Biomechanics 26, 665-676.

Omens JH, Miller TR \& Covell JW (1997). Relationship between passive tissue strain and collagen uncoiling during healing of infarcted myocardium. Cardiovascular Research 33, 351-358.

Pang Y, Wang X, Lee D \& Greisler HP (2011). Dynamic quantitative visualization of single cell alignment and migration and matrix remodeling in 3-D collagen hydrogels under mechanical force. Biomaterials 32, 3776-3783.

Petroll WM, Ma L \& Jester J V (2003). Direct correlation of collagen matrix deformation with focal adhesion dynamics in living corneal fibroblasts. Journal of Cell Science 116, 14811491. 
Sacks MS (2003). Incorporation of experimentally-derived fiber orientation into a structural constitutive model for planar collagenous tissues. Journal of Biomechanical Engineering 125, 280-287.

Safford RE, Bassingthwaighte EA \& Bassingthwaighte JB (1978). Diffusion of water in cat ventricular myocardium. Journal of General Physiology 72, 513-538.

Simpson M, Merrifield A, Landman KA \& Hughes BD (2007). Simulating invasion with cellular automata: connecting cell-scale and population-scale properties. Physical Review E 76, 111 .

Souders CA, Bowers SLK \& Baudino TA (2009). Cardiac fibroblast: the renaissance cell. Circulation Research 105, 1164-1176.

Takahashi S, Barry AC \& Factor SM (1990). Collagen degradation in ischaemic rat hearts. Biochemical Journal 265, 233-241.

Thomopoulos S, Fomovsky GM \& Holmes JW (2005). The development of structural and mechanical anisotropy in fibroblast populated collagen gels. Journal of Biomechanical Engineering 127, 742-750.

Villarreal FJ, Lew WYW, Waldman LK \& Covell JW (1991). Transmural myocardial deformation in the ischemic canine left ventricle. Circulation Research 68, 368-381.

Virag JI \& Murry CE (2003). Myofibroblast and endothelial cell proliferation during murine myocardial infarct repair. American Journal of Pathology 163, 2433-2440.

Vliegen HW, Van der Laarse A, Cornelisse CJ \& Eulderink F (1991). Myocardial changes in pressure overload-induced left ventricular hypertrophy: a study on tissue composition, polyploidization and multinucleation. European Heart Journal 12, 488-494.

Wang JH-C, Jia F, Gilbert TW \& Woo SL-Y (2003). Cell orientation determines the alignment of cell-produced collagenous matrix. Journal of Biomechanics 36, 97-102.

Wang Y, Han H-C, Yang JY, Lindsey ML \& Jin Y (2010). A conceptual cellular interaction model of left ventricular remodelling post-MI: dynamic network with exit-entry competition strategy. BMC Systems Biology 4, S5.

Weber KT (1989). Cardiac interstitium in health and disease: the fibrillar collagen network. Journal of the American College of Cardiology 13, 1637-1652.

Whittaker P, Boughner DR \& Kloner RA (1989). Analysis of healing after myocardial infarction using polarized light microscopy. American Journal of Pathology 134, 879-893.

Yarbrough WM, Mukherjee R, Escobar GP, Mingoia JT, Sample JA, Hendrick JW, Dowdy KB, McLean JE, Lowry AS, O’Neill TP \& Spinale FG (2003). Selective targeting and timing of 
matrix metalloproteinase inhibition in post-myocardial infarction remodeling. Circulation 108, 1753-1759. 


\section{CHAPTER 3: Computational Screening of Candidate Processes of Collagen Fiber Remodeling in Healing Myocardial Infarcts}

\subsection{INTRODUCTION}

In Chapter 2, we presented an agent-based model of infarct healing, which accounted for the combined influence of mechanical, structural, and chemical cues on fibroblast-mediated assembly and remodeling of the collagen fiber matrix of infarct scar tissue. By carefully comparing model predictions to experimental data, we showed that mechanical environment is likely the most important determinant of infarct collagen alignment. Mechanical cues such as uniaxial strain may cause collagen alignment via several mechanisms: by guiding alignment of fibroblasts, which then deposit collagen fibers aligned with the current cell orientation (aligned deposition) (Canty et al., 2004; Kapacee et al., 2008; Kadler et al., 2008), by guiding alignment of fibroblasts, which then generate traction forces that cause rotation of nearby collagen fibers toward alignment with the current cell orientation (rotation) (Petroll et al., 2003; Thomopoulos et al., 2005; Lee et al., 2008), or by reducing the rate of degradation of collagen fibers under tension, which promotes accumulation of collagen fibers aligned along the axis of tension (selective degradation) (Huang \& Yannas, 1977; Wyatt et al., 2009; Flynn et al., 2010; Zareian et al., 2010; Camp et al., 2011). The relative contribution of these processes toward remodeling of the collagen fiber matrix of infarct scar is unknown. Identifying the most likely processes of collagen fiber remodeling during infarct healing will improve our understanding of how interventions that alter the wound healing environment (or change the response of cells to their 
environment) will change the structural properties of infarct scar tissue, in particular, the anisotropy of the collagen fiber matrix.

The objective of this study was to determine whether aligned deposition (AD), rotation (R), or selective degradation (SD) can explain collagen remodeling during infarct healing. The parameter sensitivity analysis from the previous chapter revealed a key set of parameters - the mechanical cue weight factor $\left(\mathrm{W}_{\mathrm{m}}\right)$, the structural cue weight factor $\left(\mathrm{W}_{\mathrm{s}}\right)$, the collagen fiber generation rate coefficient $\left(\mathrm{k}_{\mathrm{cf}, \mathrm{gen}}\right)$, the collagen fiber degradation rate coefficient $\left(\mathrm{k}_{\mathrm{cf}, \mathrm{deg}}\right)$, and the collagen fiber rotation rate coefficient $\left(\mathrm{k}_{\mathrm{cf}, \mathrm{rot}}\right)$ (Table 2.2) - that determined the structure (cell alignment, collagen fiber alignment, collagen area fraction) of mature (3wk) scar were. We reasoned that the range of plausible models and input parameter values could be constrained by requiring the model predictions to simultaneously match experimental measurements of collagen area fraction $\left(\mathrm{F}_{\mathrm{cf}}\right)$, strength of collagen alignment $\left(\mathrm{MVL}_{\mathrm{cf}}\right)$, and strength of cell alignment $\left(\mathrm{MVL}_{\text {cell }}\right)$. Collagen area fraction and strength of collagen alignment were measured previously in infarct scars (Fomovsky \& Holmes, 2010; Fomovsky et al., 2012b), but we did not know the strength of cell alignment. We measured the orientations of cell nuclei in hisotological sections of three week old cryoinfarct scars and calculated the strength of cell alignment. Then we used the agent-based model of infarct healing to systematically test which processes of collagen fiber remodeling can reproduce the cell and collagen fiber structures of infarct scar tissue observed in vivo. We performed parameter sensitivity analyses on plausible models to identify differences among them, and designed novel experiments that we propose could be conducted to further test the predictive capabilities of the plausible models. 


\subsection{METHODS}

The agent-based model of infarct healing was used to test whether aligned deposition, rotation, or selective degradation can explain collagen remodeling during infarct healing. Simulations were run with each remodeling process acting in isolation, and the predictions of the models were compared among each other and against experimental data. We measured the strength of cell alignment in three week old cryoinfarcts and used this measurement as a new model validation criterion. In order to better characterize the differences among the models, we performed parameter sensitivity analyses on the models. The parameter sensitivity analyses suggested novel experiments that could further test the plausibility of each model. We simulated these experiments in order to obtain model predictions in advance of in vivo experimentation, which we propose as future work.

\section{Model Description}

The agent-based model of infarct healing described in the previous chapter was used for this study. This model could already simulate collagen remodeling via aligned deposition and rotation. In order to add selective degradation to the model, the collagen degradation rate was prescribed as a function of fiber orientation. Several studies have quantified the degradation rate of collagen fibers as a function of fiber strain (Appendix 3.A). These studies have shown that tensile strain causes no more than a 10-fold reduction of the degradation rate, which we took as an upper bound on the magnitude of the effect. For the simulations of infarcts experiencing uniaxial strain, we assumed the collagen fiber degradation rate coefficient was 10 -fold lower for 
fibers oriented along the axis of stretch relative to fibers oriented perpendicular to the axis of stretch. In order to solve for the degradation rate coefficient at orientations between these axes, we re-fit our collagen accumulation time course data, allowing the degradation rate coefficient to depend on orientation (Fig. 3.1a,b). The procedure for obtaining this solution is described in Appendix 3.B. We used systolic strains of cryoinfarcts located on the anterior wall of the left ventricle to compute fiber strain as a function of orientation and then plotted the degradation rate coefficient against fiber strain (Fig. 3.1c,d). The function we obtained for the model agreed well with published data.

\section{Measurement of Cell Orientations in Cryoinfarct Scars}

Circular cryoinfarcts were created on the anterior wall or on the apex of rat left ventricles for an earlier study (Fomovsky et al., 2012b). The infarcts located on the anterior wall deformed uniaxially along the circumferential axis of the left ventricle acutely after infarction with systolic strains of 0.07 (circumferential) and -0.01 (longitudinal), as measured by sonomicrometry. The infarcts located on the apex deformed biaxially with systolic strains of 0.02 (circumferential and 0.04 (longitudinal). Cryoinfarct scars were harvested three weeks after infarction, fixed in formalin, processed, embedded in paraffin, and cut into $7 \mu \mathrm{m}$ thick sections. For the original study, selected slides were stained with picrosirius red in order to visualize collagen fibers and measure their density and orientation. For this study, adjacent sections were stained with hematoxylin and eosin in order to visualize cell nuclei. High resolution, full-section images were acquired using the Scanscope slide scanner (Aperio). Using ImageJ, myocardium was cropped out of the images, leaving only scar tissue (Fig. 3.2a). Since hematoxylin and eosin stained cell 
nuclei dark purple and surrounding tissue bright pink, the images were converted to grayscale and thresholded in order to segment the cell nuclei from the rest of the tissue (Fig. 3.2b,c). Using morphometric functions in Matlab, ellipses were fit to all of the segmented nuclei, and their positions, sizes, aspect ratios, and orientations were measured. For each group, two to four sections per infarct in six infarcts were analyzed at an average transmural depth of $35 \%$.

\section{Simulations and Analysis}

Our first objective was to simulate healing of infarcts experiencing uniaxial strain with collagen remodeling allowed via aligned deposition only, rotation only, or selective degradation only. The mechanical cue weight factor $\left(\mathrm{W}_{\mathrm{m}}\right)$ and - for the rotation model — the collagen fiber rotation rate coefficient $\left(\mathrm{k}_{\mathrm{rot}}\right)$ were adjusted in an attempt to simultaneously satisfy all experimental observations and parameter constraints. The mechanical cue weight factor was an unconstrained parameter in the model. The collagen fiber rotation rate coefficient was limited to an upper bound of $25 \mathrm{deg} / \mathrm{hr}$, which we estimated from experiments that measured the time course of fiber alignment in fibroblast-populated collagen gels (Fig. 3.3) (Lee et al., 2008). For all models, the mechanical cue weight factor determined the strength of cell alignment. For the selective degradation model, the collagen degradation rate function (described above) determined the strength of collagen alignment. For the rotation model, the mechanical cue weight factor and the collagen fiber rotation rate coefficient determined the strength of collagen alignment. For the aligned deposition model, the mechanical cue weight factor determined the strength of collagen alignment. Experimental data used to assess the plausibility of the models were the collagen accumulation time course measured in ligation infarcts (Fomovsky \& Holmes, 2010), the 
collagen fiber orientation histogram measured in three week old circular cryoinfarcts located on the anterior wall of the left ventricle (Fomovsky et al., 2012b), and the cell orientation histogram measured in three week old circular cryoinfarcts located on the anterior wall of the left ventricle. If the aligned deposition only model, rotation only model, or selective degradation only model was not able to simultaneously reproduce these data, we considered the remodeling process unlikely to fully explain collagen fiber alignment during infarct healing.

Having narrowed the range of feasible models and parameter sets that could explain the experimental data, we performed parameter sensitivity analyses to identify novel experiments that could be used to further differentiate and validate the remaining models. The parameter sensitivity analyses were performed as described in the previous chapter. Because of our interest in processes of collagen fiber alignment for this study, we focused on the sensitivity of the strength of collagen fiber alignment to changes in model input parameters. If the sensitivity to an input parameter differed substantially between two models, it was flagged as a potential line of future investigation.

We narrowed the list of flagged input parameters to those amenable to experimental manipulation and designed experiments for which the remaining candidate models predicted different outcomes. In order to design these experiments, we ran infarct healing simulations with each of the remaining models, increased or decreased one of the flagged input parameters at several time points after infarction, and compared how the models responded to the interventions. We then identified the timing of each intervention and the corresponding output measurement that best exposed the different predictions of the models. 


\subsection{RESULTS}

\section{Organization of Cells in Cryoinfarct Scars}

We measured the strength of cell alignment in cryoinfarct scars for use as additional experimental data for model validation. We used a semi-automated image processing algorithm implemented in Matlab to measure the orientations of hematoxylin-stained cell nuclei in histological sections taken from the same cryoinfarct samples used previously to measure collagen fiber orientations. Cryoinfarcts located on the apex of the left ventricle that experienced equibiaxial strain during healing had randomly oriented cells with a mean vector length of 0.03 +/- 0.10. Cryoinfarcts located on the anterior wall of the left ventricle that experienced uniaxial strain along the circumferential axis during healing had cells oriented along the circumferential axis with a mean angle of $-14+/-13$ degrees and mean vector length of $0.30+/-0.15$. The cell orientation histograms were very similar to the collagen fiber orientation histograms measured previously (Fig. 3.4). This analysis also provided measurements of nucleus size, nucleus aspect ratio, and cell density (Table 3.1).

\section{Individual Processes of Collagen Fiber Remodeling}

We sought to determine whether aligned deposition, rotation, or selective degradation was able to explain collagen fiber remodeling during infarct healing. Model simulations were run with circular infarcts, a circumferentially oriented initial matrix, and uniaxial strain acting along the 
circumferential axis. The models allowed collagen fiber remodeling via only aligned deposition (AD), only rotation $(\mathrm{R})$, or only selective degradation (SD). Input parameters were adjusted to find the best match of each model to experimental data obtained from infarct scars. The AD model and $\mathrm{R}$ model were able to reproduce the experimentally measured collagen mean vector length 3 weeks after infarction (Fig. 3.5a). The SD model failed to reproduce the collagen mean vector length 3 weeks after infarction (Fig. 3.5a).

The AD model and $\mathrm{R}$ model were compared to the baseline model presented in the previous chapter, which allowed collagen fiber remodeling via both aligned deposition and rotation $(A D+R)$. All three models predicted similar collagen mean vector length time courses and predicted collagen fiber orientation distributions 3 weeks after infarction that agreed with experimental data (Fig. 3.5c,d). The AD model and R model also predicted the experimentally measured cell mean vector length and cell orientation distribution 3 weeks after infarction (Fig. 3.5e,f). The $A D+R$ model predicted a lower cell mean vector length over the time course of healing and the cell orientation distribution 3 weeks after infarction fell over a standard deviation away from the mean of the experimental data (Fig. 3.5e,f).

\section{Parameter Sensitivity Analysis}

We sought to identify differences between the AD model and R model that were not apparent from simulating a single infarct healing scenario (circular cryoinfarct located on the anterior wall of the left ventricle). We performed parameter sensitivity analyses on the AD and R models as described in the previous chapter and focused on the sensitivity of the collagen mean vector 
length to changes in model input parameters. Sensitivities to the initial collagen area fraction, collagen turnover rate coefficients, and collagen rotation rate coefficient differed between the $\mathrm{AD}$ and $\mathrm{R}$ models (Fig. 3.6). We focused on the collagen turnover rate coefficients because these rates are potentially amenable to experimental manipulation. For the AD model, the collagen mean vector length was insensitive to the collagen degradation rate coefficient and slightly sensitive to the collagen generation rate coefficient (Fig. 3.6a). For the R model, the sensitivities of the collagen mean vector length to the degradation and generation rate coefficients were high in comparison to the sensitivities calculated for the AD model (Fig. 3.6b).

\section{Design of Novel Experiments for Model Validation}

We designed experiments that could provide evidence supporting the AD model or R model. The parameter sensitivity analysis suggested that the AD and R models would predict different collagen structures in response to a change in the rate of collagen degradation or a change in the rate of collagen deposition during infarct healing. We also realized that the rates of collagen fiber remodeling might differ between the two models because the AD model only allows remodeling via replacement of a fraction of the total number of fibers, while the R model allows reorientation of all fibers. Based on these observations, we designed experiments for which the $\mathrm{AD}$ and $\mathrm{R}$ models predicted different outcomes. 


\section{Perturbation of the Rate of Collagen Degradation}

Model simulations were run with circular infarcts, a circumferentially oriented initial matrix, and uniaxial strain acting along the circumferential axis. At one or three weeks after infarction, the collagen fiber degradation rate was scaled by a factor ranging from $1 / 10$ to 10 , and the simulation was continued until six weeks after infarction. For the AD and R models, increasing or decreasing the collagen fiber degradation rate coefficient caused the collagen area fraction to approach a lower or higher steady state value, respectively (Fig. 3.7a,b). For the R model, increasing or decreasing the degradation rate coefficient also cause the collagen mean vector length to approach a lower or higher steady state value, respectively (Fig. 3.7c,d). For the AD model, changing the degradation rate coefficient had no effect on the collagen mean vector length time course (Fig. 3.7c,d). Three weeks after beginning a set of interventions, the R model predicted a positive correlation between collagen mean vector length and collagen area fraction, whereas the AD model predicted no relationship (Fig. 3.7e,f). The responses of the models were similar whether the intervention began one week or three weeks after infarction.

\section{Perturbation of the Rate of Collagen Generation}

Model simulations were run with circular infarcts, a circumferentially oriented initial matrix, and uniaxial strain acting along the circumferential axis. At one or three weeks after infarction, the collagen fiber generation rate was scaled by a factor ranging from 1/10 to 4 (above 4 the

calculated steady state area fraction exceeds 1), and the simulation was continued until six weeks after infarction. For the $\mathrm{AD}$ and $\mathrm{R}$ models, increasing or decreasing the collagen fiber generation 
rate coefficient caused the collagen area fraction to approach a higher or lower steady state value, respectively (Fig. 3.8a,b). For the $\mathrm{AD}$ and $\mathrm{R}$ models, increasing or decreasing the generation rate coefficient beginning one week after infarction caused the collagen mean vector length to transiently decrease or increase relative to the baseline prediction (Fig. 3.8c). For the R model, increasing or decreasing the generation rate coefficient beginning three weeks after infarction also caused the collagen mean vector length to transiently decrease or increase relative to the baseline prediction (Fig. 3.8d). For the AD model, changing the generation rate beginning 3 weeks after infarction had a negligible effect on the predicted collagen mean vector length time course (Fig. 3.8d). For the set of interventions begun one week after infarction, both models predicted a negative correlation between collagen mean vector length and collagen area fraction one week after intervention (Fig. 3.8e). For the set of interventions begun three weeks after infarction, the $\mathrm{R}$ model predicted a negative correlation between collagen mean vector length and collagen area fraction one week after intervention, whereas the AD model predicted no relationship (Fig. 3.8f).

\section{Perturbation of Mechanical Environment}

Model simulations were run with circular infarcts, a circumferentially oriented initial matrix, and equibiaxial strains. At $7,10.5,14,21$, or 42 days after infarction the strain pattern was changed from equibiaxial to uniaxial with strain acting along the circumferential axis. For the AD and R models, the strength of collagen fiber alignment initially decreased as expected in the absence of a mechanical guidance cue. At the time of the mechanical intervention, the collagen mean vector length stopped decreasing and began increasing in response to the mechanical guidance cue (Fig. 
3.9a). When the mechanical environment was changed one week after infarction, the AD model predicted faster remodeling of the collagen matrix than the $\mathrm{R}$ model. When the mechanical environment was changed three weeks after infarction, the $\mathrm{R}$ model predicted faster remodeling of the collagen matrix than the AD model. We determined time constants for the remodeling response by fitting exponential saturation functions to the collagen mean vector length time courses (post-intervention). For the AD model, the rate of remodeling of the collagen matrix decreased as the timing of the intervention increased, whereas the $\mathrm{R}$ model predicted a rate of remodeling independent of the time of mechanical intervention (Fig. 3.9b). Alternatively, the AD model predicted a positive correlation between the remodeling time constant and collagen area fraction (at the time of the intervention), whereas the $\mathrm{R}$ model predicted no relationship (Fig. 3.9c).

\section{Candidate Experiments for Model Validation}

The model perturbation studies motivate three experiments where the AD and R models predict different outcomes (Table 3.2). Experiment 1: create circular cryoinfarcts located on the anterior wall of the left ventricle (uniaxial strain along circumferential axis, promotes collagen alignment), change the collagen degradation rate beginning 1 week after infarction (Group 1: decrease degradation rate, Group 2: increase degradation rate, Group 3: sham), measure collagen area fraction and collagen mean vector length 4 weeks after infarction, test if collagen area fraction differs between groups, test if collagen mean vector length differs between groups. Experiment 2: create circular cryoinfarcts located on the anterior wall of the left ventricle, change the collagen generation rate beginning 3 weeks after infarction (Group 1: decrease 
generation rate, Group 2: increase generation rate, Group 3: sham), measure collagen area fraction and collagen mean vector length 4 weeks after infarction, test if collagen area fraction differs between groups, test if collagen mean vector length differs between groups. Experiment 3: create ligation infarcts (equibiaxial strain, does not promote collagen alignment), change strain to uniaxial along circumferential axis at different times after infarction (Group 1: 1 week after infarction, Group 2: 3 weeks after infarction, Group 3: 1 week sham, Group 4: 3 week sham), measure collagen mean vector length versus time after intervention, test if collagen remodeling time constant differs between groups.

\subsection{DISCUSSION}

In the previous chapter, we presented an agent-based model of infarct healing, which accounted for the combined influence of mechanical, structural, and chemical cues on fibroblast-mediated assembly and remodeling of the collagen fiber matrix of infarct scar tissue. One over-arching goal of this work was to understand how interventions that change the mechanical, structural, or chemical environment of an infarct (or change the response of cells to their environment) will change the properties of infarct scar tissue, in particular, the anisotropy of the collagen fiber matrix. We realized that the outcomes of such interventions could depend on the process by which collagen fibers are aligned during infarct healing, which motivated us to use the agentbased model of infarct healing to test whether experimental evidence agrees with any of three candidate processes of collagen remodeling, which we termed aligned deposition (AD), rotation (R), and selective degradation (SD). 


\section{Organization of Cells in Cryoinfarct Scars}

The parameter sensitivity analysis from the previous chapter revealed that a measurement of the alignment of cells in infarct scar could help constrain the range of plausible models and input parameter values. We estimated cell alignment by measuring the orientations of hematoxylinstained cell nuclei in histological sections of three week old cryoinfarct tissue. It was possible to measure the orientation of the cell nuclei because their aspect ratio was $2.8+/-0.2$. The strong correlation of nucleus orientation with cell orientation, especially for spindle-shaped cells such as fibroblasts, has been known since 1921 (Champy \& Carleton, 1921) and was recently quantified by McKee et. al. (2011). Our quantitative measure of cell alignment confirmed the strong correlation between cell and collagen fiber orientations that is readily apparent from microscopic inspection of infarct tissues.

\section{Selective Degradation}

Selective degradation refers to a process by which a matrix of collagen fibers becomes aligned in the direction of uniaxial mechanical tension because the rate of enzymatic degradation of collagen fibers decreases as tension acting on the fibers increases. Thus, fibers oriented away from the direction of tension will have short lifetimes, fibers oriented along the direction of tension will have long lifetimes, and the long-lived fibers will dominate the structure over time. Selective degradation has been demonstrated using individual collagen fibers (Camp et al., 2011), collagen gels (Bhole et al., 2009; Flynn et al., 2010), and excised collagenous tissues such as cornea and tendon (Wyatt et al., 2009; Zareian et al., 2010), but it is unknown if this effect is 
important in vivo. These studies have reported $25 \%$ to 10 -fold reductions of the degradation rate of collagen fibers in response to uniaxial tension.

We simulated healing of infarcts experiencing uniaxial tensile strain, assumed the degradation rate of collagen fibers decreased 10-fold as a function of orientation from perpendicular to parallel to the axis of tension, and found that selective degradation alone failed to reproduce the strength of collagen fiber alignment observed in three week old cryoinfarct scars. This is because collagen degradation has a weak effect on the net collagen fiber structure relative to collagen deposition during the accumulation phase that occurs over the first few weeks after infarction. We conclude that selective degradation is more likely to be an important determinant of collagen fiber alignment in situations where collagen content is stable or declining, not during the early phase of wound healing that demands rapid matrix assembly.

\section{Aligned Deposition}

Aligned deposition refers to a process by which a matrix of aligned collagen fibers is generated by fibroblasts that align in response to an environmental guidance cue (e.g. uniaxial mechanical tension) and deposit new collagen fibers along the axis of cell alignment (Wang et al., 2003; Kapacee et al., 2008). Histological studies of tendon and engineered tendon mimics suggest that fibroblasts can curve shape their plasma membrane into parallel arrays of troughs or channels (termed fibripositors) that serve to direct assembly of secreted collagen monomers into fibers aligned with the long axis of the cell (Canty et al., 2004; Bayer et al., 2010). Other studies have shown that fibroblasts seeded on micro-grooved substrates align parallel to the grooves and then 
assemble collagen matrices with fibers co-aligned with the cells. Because these studies are correlative, we cannot rule out the possibility that fibroblasts and collagen fibers became coaligned via some other process.

We simulated healing of infarcts experiencing uniaxial tensile strain, implemented aligned deposition, and found that aligned deposition alone reproduced the strengths of collagen alignment and cell alignment observed in three week old cryoinfarct scars. To obtain this result, we chose values of the collagen degradation and generation rate coefficients that reproduced the collagen accumulation data and chose a value of the mechanical cue weight factor that reproduced the cell alignment data. The model also reproduced the collagen alignment data because, for aligned deposition, the strength of collagen alignment must eventually converge to the strength of fibroblast alignment, and convergence must occur on pace with the rate of accumulation or rate of replacement of collagen fibers.

The behavior of the AD model is relatively simple because aligned deposition enforces coupling of cell and collagen alignment. First, this coupling suggests that if aligned deposition occurs during infarct healing, then it must be the dominant process of collagen remodeling. By reproducing the cell mean vector length, the $\mathrm{AD}$ model was forced to reproduce the collagen mean vector length as well. This left no "room" for another process of collagen fiber alignment (e.g. AD+R), which would cause the model to over-predict the strength of collagen alignment. Second, aligned deposition caused the strength of collagen alignment in infarct scar to be insensitive to most perturbations. Over the long term, the strength of collagen alignment 
depended only on the strength of cell alignment (determined by the strength of the mechanical guidance cue).

\section{Rotation}

Rotation refers to a process by which a matrix of collagen fibers becomes aligned by fibroblasts that align in response to an environmental guidance cue (e.g. uniaxial mechanical tension) and generate traction forces that cause nearby collagen fibers to rotate toward the axis of cell alignment. We consider fibroblast-mediated rotation of collagen fibers to be a phenomenologic representation of a process of local rearrangement of fibers that likely has more complex kinematics. In vitro studies of fibroblasts in collagen gels have shown that spindle-shaped fibroblasts exert traction forces predominantly along their axis of elongation (Rape et al., 2011), and these traction forces cause nearby collagen fibers to become aligned with the cell (Petroll et al., 2003; Petroll \& Ma, 2003; Pang et al., 2011). Furthermore, uniaxial tension applied to fibroblast-populated collagen gels has been shown to cause alignment of fibroblasts, which generate traction forces that deform the initially isotropic collagen matrix into an aligned structure (Thomopoulos et al., 2005; Lee et al., 2008). However, it is unknown if traction forces generated by fibroblasts can similarly deform the infarct collagen matrix into an aligned structure.

We simulated healing of infarcts experiencing uniaxial tensile strain, programmed fibroblasts to rotate nearby collagen fibers toward alignment with the current orientation of the cell, and found that rotation alone reproduced the collagen accumulation time course observed in infarct scars 
and reproduced the strengths of collagen alignment and cell alignment observed in three week old infarct scar. To obtain this result, we chose values of the collagen degradation and generation rate coefficients that reproduced the collagen accumulation data, chose a value of the mechanical cue weight factor that reproduced the cell alignment data, and then chose a value of the collagen fiber rotation rate coefficient that reproduced the collagen alignment data. The value of the collagen fiber rotation rate coefficient that was required fell well within the upper bound we estimated from literature data.

The behavior of the $\mathrm{R}$ model is more complex than the behavior of the AD model because rotation allows decoupling of cell and collagen alignment. First, rotation could act in combination with selective degradation (but not with aligned deposition, as explained above). Second, rotation allowed the strength of alignment of infarct scar to be sensitive to perturbation of the collagen turnover rate coefficients. For the $\mathrm{R}$ model, there is an antagonistic relationship between collagen fiber rotation and collagen fiber turnover. Increasing the collagen fiber degradation rate decreases the time available for fibers to be rotated into alignment. Increasing the collagen fiber generation rate transiently increases the proportion of randomly oriented fibers added to the collagen matrix, which transiently decreases the strength of alignment of the matrix. Over the long term, the strength of collagen alignment depended on the strength of cell alignment (determined by the strength of the mechanical guidance cue) and on the collagen fiber degradation rate. 


\section{Candidate Experiments for Model Validation}

The model perturbation studies motivate three experiments where the AD and R models predict different outcomes (Table 3.2). We did not have sufficient experimental data at the time of this study to conclude whether aligned deposition or rotation agreed better with the observed collagen fiber remodeling during infarct healing. However, we had enough experimental data to constrain every parameter in the $\mathrm{AD}$ and $\mathrm{R}$ models. Thus, the three experiments proposed here can be used to further test the plausibility of the AD and R models. Experiment 1 is designed to test if the collagen mean vector length of infarcts experiencing uniaxial strain (circular cryoinfarct located on the anterior wall of the left ventricle) is sensitive to perturbation of the collagen degradation rate. Experiment 2 is designed to test if the collagen mean vector length of infarcts experiencing uniaxial strain is sensitive to perturbation of the collagen generation rate. Experiment 3 is designed to test if the collagen fiber remodeling time constant of infarcts experiencing biaxial strain (ligation infarct or circular cryoinfarct located on the apex of the left ventricle) depends on the timing of a switch of the mechanical environment from equibiaxial to uniaxial strain.

We suggest attempting Experiment 1 first because it has the simplest design and requires the least resources. Experiment 1 involves creating circular cryoinfarcts on the anterior walls of rat hearts, perturbing the collagen fiber degradation rate for a period of time after infarction, and then measuring the strength of collagen alignment in the infarct scars. The experimental treatment would either decrease the collagen fiber degradation rate (Group 1) or increase the degradation rate (Group 2). Changing the collagen fiber degradation rate could be accomplished 
through many different means. MMPs could be delivered to the infarct to increase the rate of collagen degradation. MMP blocking antibodies or small molecule inhibitors of MMPs could be delivered to the infarct to decrease the rate of collagen degradation (Spinale, 2007). Molecular genetics techniques could be employed to modulate expression of MMPs or TIMPs (Creemers $e t$ al., 2001; Vanhoutte et al., 2006). The aim of the experiment is to test if the strength of collagen alignment differs between groups. The key design variables are the sample size and the timing, duration, and magnitude of the treatment.

In order to assess the feasibility of this experiment, we performed a power analysis to calculate the sample size required for a two-tailed t-test with a significance level of 0.05 , power of 0.95 , and standard deviation of 0.234 (average of the mean vector length standard deviations from our lab's ligation infarct and cryoinfarct studies). We calculated sample size as a function of the difference between mean vector lengths predicted for different combinations of down- and upregulation of the collagen fiber degradation rate, and we selected the combinations that required a sample size of 10 . We performed the power analysis for interventions lasting from Week 1 to Week 4, Week 1 to Week 6 , or Week 3 to Week 6 after infarction. The power analysis showed that there are many combinations of intervention magnitudes and intervention times that are possible for a sample size of 10 (Fig. 3.10). Perhaps the most attractive option would be to apply the intervention from Week 1 to Week 6 . If a 10-fold decrease of the degradation rate could be achieved for Group 1, then control would suffice for Group 2. We would only need a method of blocking collagen degradation, without needing a method of increasing the rate of collagen degradation. Local delivery of a broad spectrum, small molecule MMP inhibitor could suffice. 
In contrast to Experiment 1, Experiment 2 would require a larger sample size because the strength of collagen alignment is less sensitive to perturbation of the collagen fiber generation rate than it is to perturbation of the collagen fiber degradation rate. Also, Experiment 2 is more susceptible to error because it requires measuring a transient response to the intervention, which could be missed if the model has not predicted the dynamics of the response exactly.

Experiment 3 would require an even larger sample size because it seeks to quantify a collagen fiber remodeling time constant for each group, which requires measurement of the collagen fiber structure at multiple time points for each group. Even if this remodeling time constant could be estimated by quantifying the collagen fiber structures at just two time points for each group, the total number of animals needed would be approximately double compared to the number needed for Experiment 1. Furthermore, although our lab has developed techniques for sewing fabric patches to the surface of the left ventricle in order to anisotropically reinforce infarcts, for this experiment, rats would be subjected to two invasive procedures (infarct then patch), which should be avoided on ethical grounds if satisfactory alternatives exist.

\section{Model Insights and Significance}

We motivated this work by arguing that we must know which remodeling processes play a role during infarct healing because our choice of remodeling process may affect our understanding of how the structural (and mechanical) properties of infarct scar tissue will adapt to various conditions in vivo. We have shown that our choice of remodeling process matters for many, but not all infarct healing scenarios. On the surface, the $\mathrm{AD}$ and $\mathrm{R}$ models appeared to be 
interchangeable. They predicted very similar cell and collagen fiber structures over the time course of healing for the baseline model conditions. However, the parameter sensitivity analyses revealed some unique features of each model.

For example, the long term response of the models to a change of environmental guidance cues was similar, but the dynamics of the response were different. In particular, early after infarction the aligned deposition process had a smaller remodeling time constant than the rotation process. Therefore, whether aligned deposition or rotation is a dominant process of collagen fiber remodeling affects what we expect will happen to the structural (and mechanical) properties of the infarct in response to variations of environmental conditions early after infarction. Such variations may arise from variability of a patient's innate biology, habits, and age (Jugdutt, 2008a), or from a therapeutic intervention (Jugdutt, 2008b). The aligned deposition model could predict rapid structural remodeling of the infarct that could have significant (positive or negative) consequences, such as preserving, enhancing, or diminishing the structural integrity of the infarct, whereas the rotation model could predict a relatively damped response with insignificant consequences.

Currently, few experimental interventions for treatment of myocardial infarction are being developed for use earlier than two weeks after infarction because of the risk of complications such as rupture. However, it is during this time that much maladaptation of the infarct occurs, such as thinning and expansion (Weisman et al., 1985). Thus, we expect interventions such as injection of polymers or cells or application of supportive materials to have greatest potential benefit early after infarction (Rane \& Christman, 2011; Fomovsky et al., 2012a). By furthering 
our understanding of how the structure of infarct scar tissue can remodel, this work can help identify and mitigate the risks of intervention.

For the purpose of long term regulation or prediction of the collagen fiber structure of infarct scar, the most significant difference between the AD and R models is that, for the R model, changing the collagen fiber degradation rate will change the density and anisotropy of the collagen fiber matrix. For the AD model, changing the collagen fiber degradation rate will only change the density of the collagen fiber matrix. If aligned deposition is dominant during infarct healing, then regulating or predicting the collagen fiber structure of infarct scar is straightforward in principle. Changing the collagen fiber generation rate or degradation rate will change collagen content, and changing the strength and/or orientation of guidance cues will change collagen alignment. If rotation is dominant during infarct healing, changing the collagen fiber degradation rate will also change the strength of collagen alignment. This is potentially an unintended (and possibly beneficial) consequence of interventions, such as MMP inhibition, that change the rate of collagen fiber turnover, which re-iterates the importance of determining which collagen fiber remodeling process dominates during infarct healing. It is interesting to speculate that this effect may explain why rat cryoinfarcts and pig infarcts experienced similar uniaxial strains during healing, but pig infarcts had higher collagen content and stronger collagen fiber alignment (Holmes et al., 1997; Fomovsky et al., 2012b). 


\section{Model Limitations}

Our conclusions about the roles and possible effects of aligned deposition, rotation, and selective degradation during infarct healing depend on the way we represented these processes in the model. The rotation model represented (in abstraction) re-arrangement of collagen fibers caused by the traction forces fibroblasts exert on nearby matrix. As the density and crosslinking of collagen fibers increases over time after infarction, we expect the collagen matrix to stiffen, which should make it more difficult for fibroblasts to deform their local matrix. Indeed, studies have shown that the rate of fibroblast-mediated compaction of collagen gels depends inversely on collagen density (Loftis et al., 2003). However, in the rotation model, the collagen fiber rotation rate did not depend on collagen density. If the next set of experiments fails to support the rotation model, then dependence of the collagen fiber rotation rate on collagen density should be tested as a possible explanation.

For the aligned deposition model, we assumed the orientation of newly deposited collagen fibers was exactly equal to the current orientation of the cell. In reality, collagen fibers may be deposited with an orientation bias that is not nearly as strong. This would imply that aligned deposition alone could not account for the strength of collagen alignment observed in infarct scars. Again, only if the next set of experiments fails to support the aligned deposition model should we consider a more complex model.

For the selective degradation model, we assumed the collagen fiber degradation rate depended on the systolic strain acting on the infarct, which was calculated using end diastole as a reference 
state. Literature studies reported reductions of the degradation rate of collagen fibers in response to tension relative to an unloaded state. We estimate the infarct was subjected to longitudinal strain of $6 \%$ and circumferential strain of $11 \%$ at end diastole relative to its unloaded state (Omens et al., 1993). These strains indicate the infarct was under tension in all directions, which suggests that the selective degradation model over-predicted the magnitude of the effect that selective degradation could have in an infarct.

The collagen generation and degradation rate coefficients used in the models were determined from collagen accumulation time courses obtained from a ligation infarct study (Fomovsky \& Holmes, 2010), whereas the cell and collagen alignment data was obtained from cryoinfarcts (Fomovsky et al., 2012b). If the collagen accumulation rate for the cryoinfarcts was much faster than measured for the ligation infarcts, the selective degradation model could predict stronger collagen alignment, but as discussed above, we expect that we have over-estimated the magnitude of the effect that selective degradation could have in an infarct. We have also assumed that the collagen generation and degradation rate coefficients are constant over time. If these rates decrease over time, then the sensitivity of the collagen area fraction and strength of collagen alignment to perturbation of these rates would be lower. In the proposed experiments, we can check if the intervention is having any effect by measuring collagen area fraction. It may be necessary to limit the experimental treatment to the first few weeks after infarction when MMP activity and the rate of collagen synthesis are high. 


\subsection{CONCLUSION}

Our results suggest that aligned deposition or rotation could be the dominant process of collagen remodeling during infarct healing. Based on available evidence, selective degradation is unlikely to be the dominant process of collagen remodeling during infarct healing, but could act in combination with rotation. Although we were unable to conclude whether aligned deposition or rotation is more consistent with experimental data, by measuring the strength of cell alignment in cryoinfarct scars, we obtained sufficient data to constrain every parameter in the AD and $\mathrm{R}$ models. Parameter sensitivity analyses revealed distinct differences between the $\mathrm{AD}$ and $\mathrm{R}$ models that highlight the importance of understanding which remodeling processes play a role during infarct healing. For the AD model, the strength of collagen alignment was relatively insensitive to the collagen fiber turnover rate parameters, and the collagen fiber remodeling rate depended on collagen content. In contrast, for the R model, the strength of collagen alignment was sensitive to the collagen fiber turnover rate parameters, and the collagen fiber remodeling rate did not depend on collagen content. We exploited the differences between these models to design three experiments for model validation. We recommend perturbing the collagen fiber degradation rate during infarct healing and testing if this changes the strength of collagen fiber alignment, which the $\mathrm{R}$ model predicts but the AD model does not. Because the collagen fiber structure of infarct scar affects the mechanical properties of the infarct, which in turn affect heart function, properly predicting collagen fiber remodeling is important for design of interventions that treat myocardial infarction. 


\subsection{FIGURES AND TABLES}

A

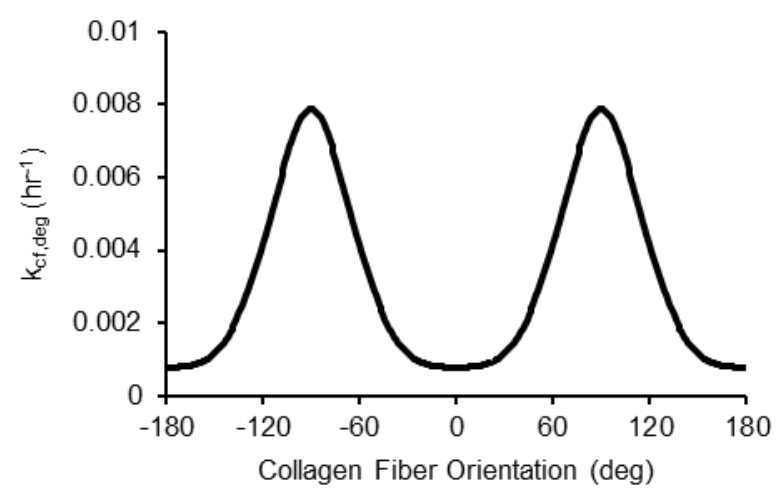

$\mathrm{C}$

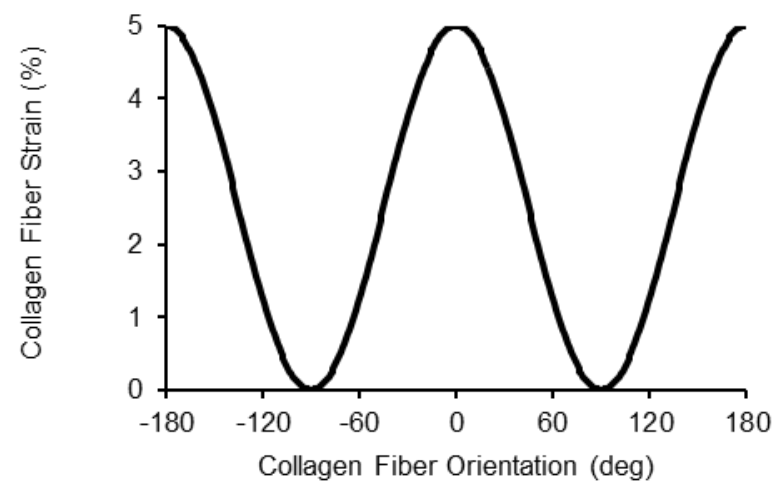

B

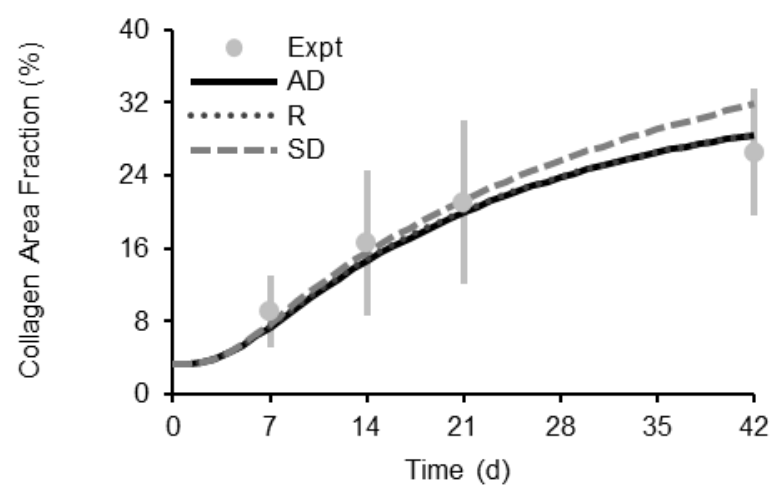

$\mathrm{D}$

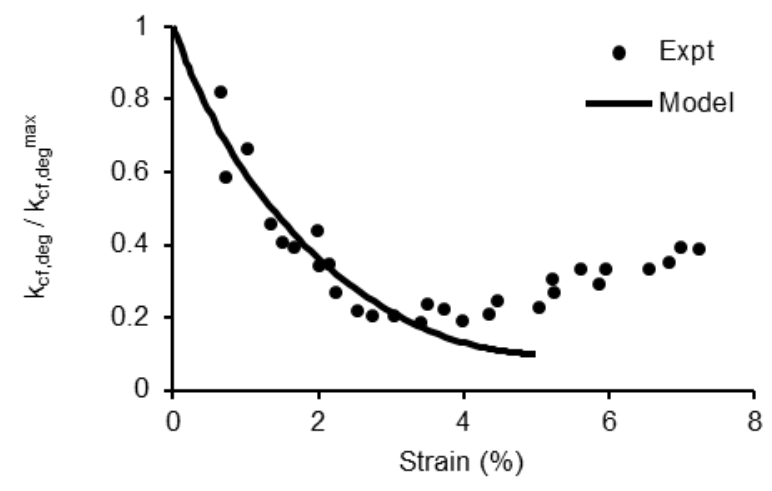

Figure 3.1. Collagenase mechano-chemistry. Seven studies have quantified the effect of mechanical tension on the rate of enzyme-mediated degradation of collagen fibers (Appendix 3.A). A. We simulated the largest effect reported in the literature by prescribing a 10-fold decrease of the collagen fiber degradation rate coefficient as a function of fiber orientation from perpendicular (+/- 90 degrees) to parallel (0 and 180 degrees) to the axis of stretch. B. We adjusted the mean degradation rate coefficient so the model (SD) would match the collagen accumulation time course from the ligation study, similar to the other models (AD and R). $\mathbf{C}$. We used the strain tensor from our cryoinfarct data to compute fiber strains from fiber orientations in order to plot the collagen degradation rate coefficient as a function of fiber strain. D. The data reported by Huang and Yannas (1977) best represented the collective observations of the literature studies. As the magnitude of tensile strain applied to the collagenous material increased, the rate of collagen degradation decreased, reached a minimum, and then began to increase. The model function reproduced the initially downward trending degradation rate reported by Huang and Yannas. 
A
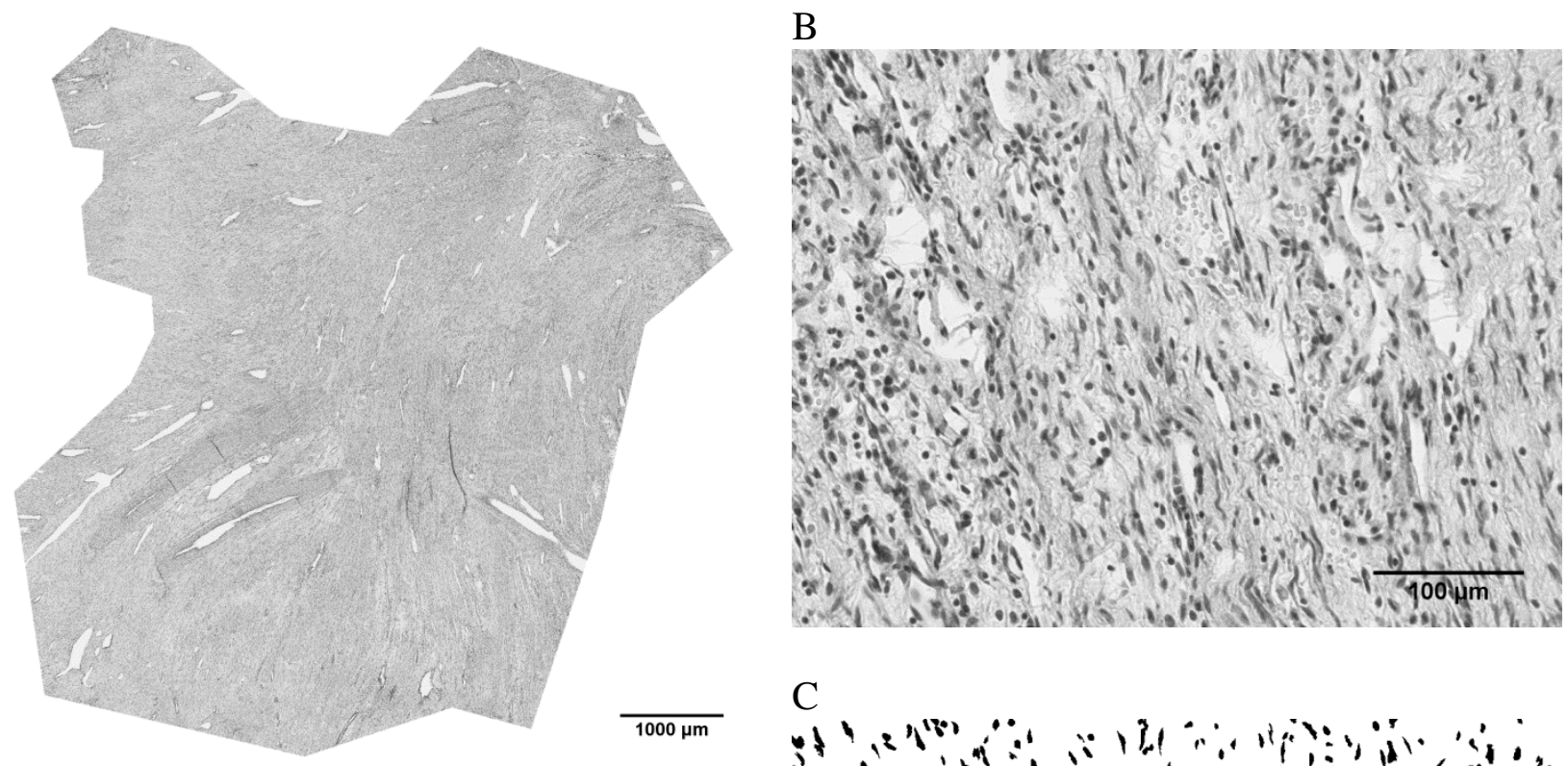

C

Figure 3.2. Example images of $H \& E$ stained cryoinfarct tissue for measurement of cell alignment. A. Whole sections were imaged using the Scanscope slide scanner (Aperio) then cropped to retain only infarct tissue. B. Example sub-region illustrating darkly-stained cell nuclei. C. After thresholding and filtering extremely small and extremely large objects, the position, size, aspect ratio, and orientation of all cell nuclei were quantified using morphometric functions in Matlab.

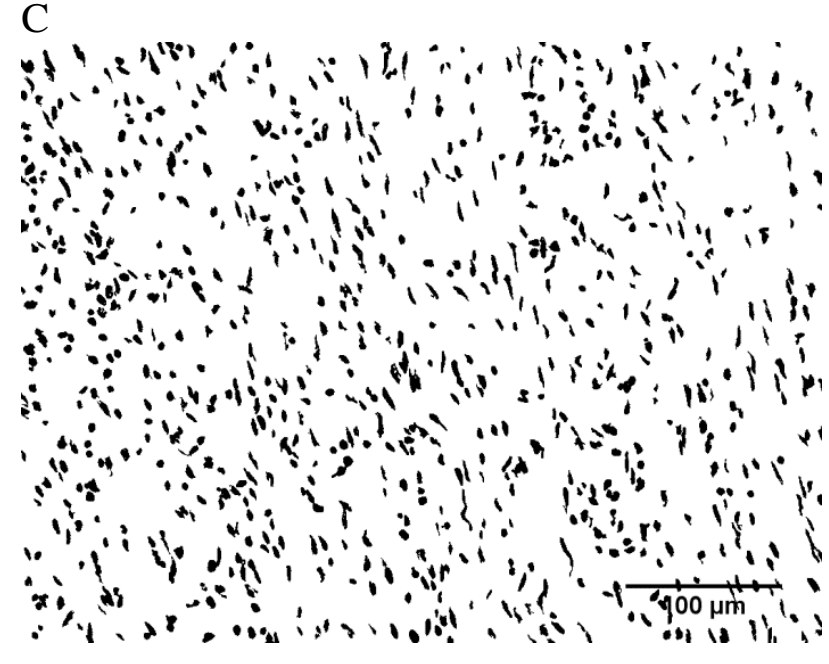


A

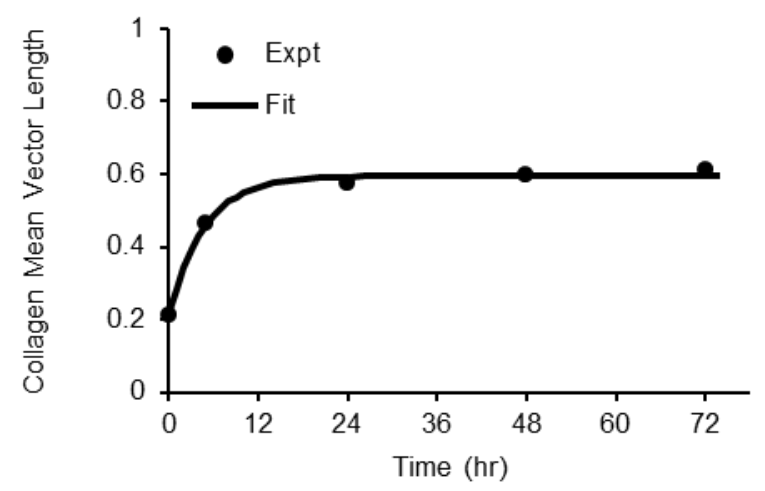

B

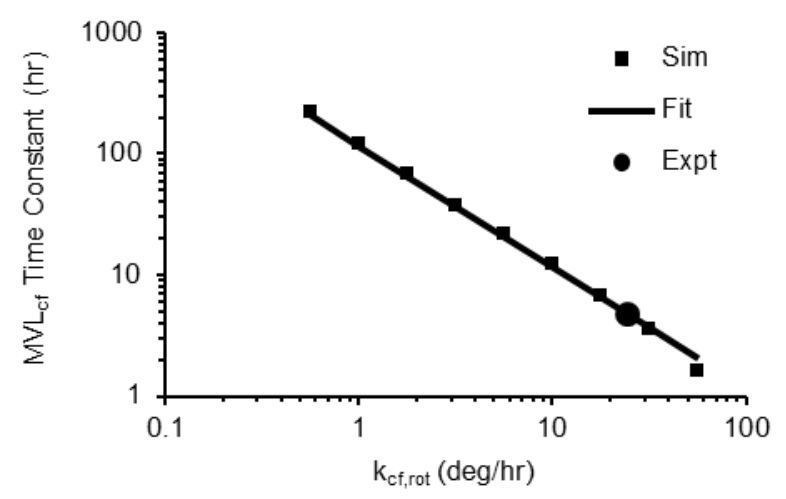

Figure 3.3. Collagen fiber rotation rate coefficient. Experimental data that could constrain the magnitude of the collagen fiber rotation rate coefficient was needed in order to assess the plausibility of models that allowed remodeling of collagen fiber orientations only by the rotation process. A. Lee et al. (2008) seeded fibroblasts in initially isotropic collagen gels, loaded the gels uniaxially, and quantified the strength of collagen fiber alignment as fibroblasts remodeled the collagen matrix over time. The collagen mean vector length time course was fit to an exponential saturation function in order to derive a time constant $\tau \sim 5 \mathrm{hr}$. B. For a range of collagen fiber rotation rate coefficients, remodeling of the collagen gel was simulated and the collagen mean vector length time course was fit to an exponential saturation function in order to derive a time constant. The time constant was inversely proportional to the collagen fiber rotation rate coefficient. From this relationship, the rotation rate coefficient corresponding to the experimental data was calculated as $\mathrm{k}_{\mathrm{cf}, \mathrm{rot}}{ }_{\max }=25 \mathrm{deg} / \mathrm{hr}$. This was interpreted as an upper bound on the in vivo rotation rate coefficient, based on the assumption that low density, non-covalently crosslinked gels created in vitro are more easily deformed than matrices produced in vivo. 
A. Anterior Wall

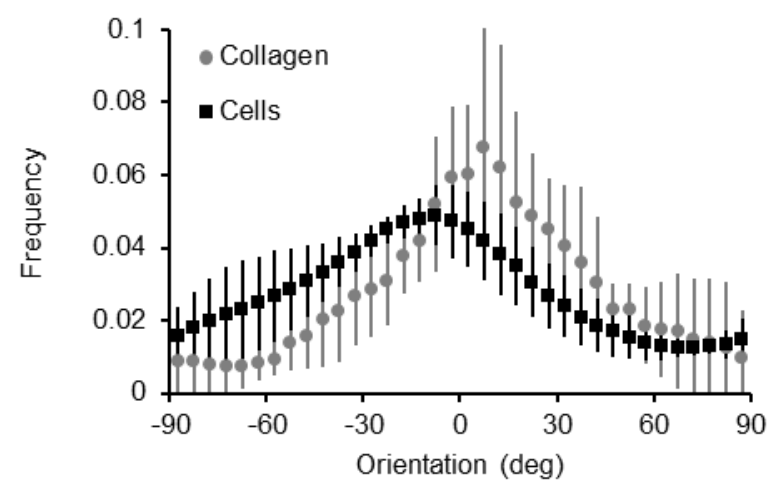

B. Apex

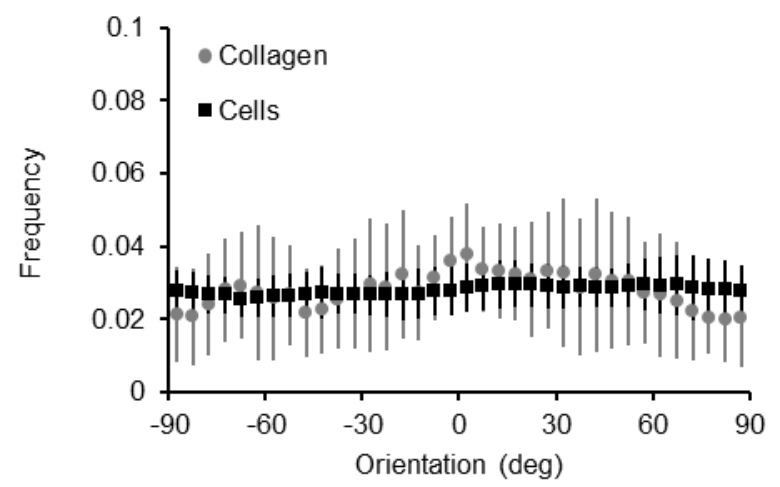

Figure 3.4. Cell and collagen alignment in 3 week old circular cryoinfarct scars located on the apex or anterior wall of rat left ventricles. The orientations of hematoxylin-stained cell nuclei were measured from sections of cryoinfarct scar tissue at approximately $35 \%$ transmural depth and orientation histograms were calculated. A. Cryoinfarct scars located on the anterior wall of the left ventricle that stretched uniaxially along the circumferential direction ( 0 degrees) had cells aligned along the circumferential direction. B. Cryoinfarct scars located on the apex of the left ventricle that stretched equibiaxially had no cell alignment. The cell orientation histograms agreed closely with the collagen fiber orientation histograms of these infarcts that were measured previously (Fomovsky et. al., 2012). 
A

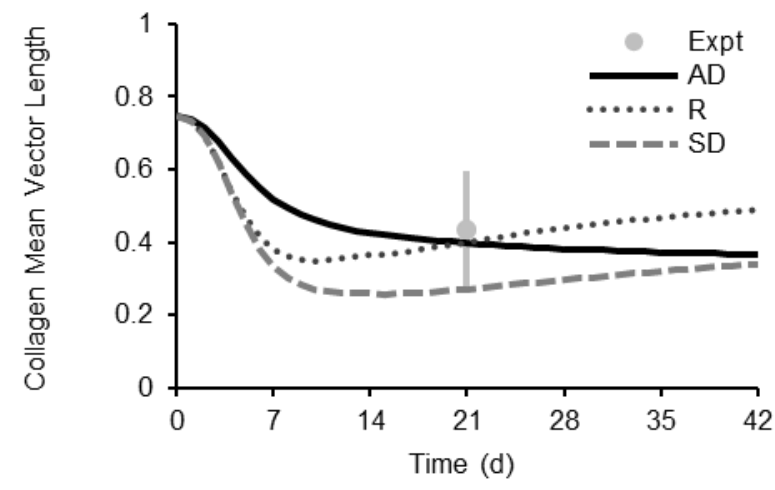

$\mathrm{C}$

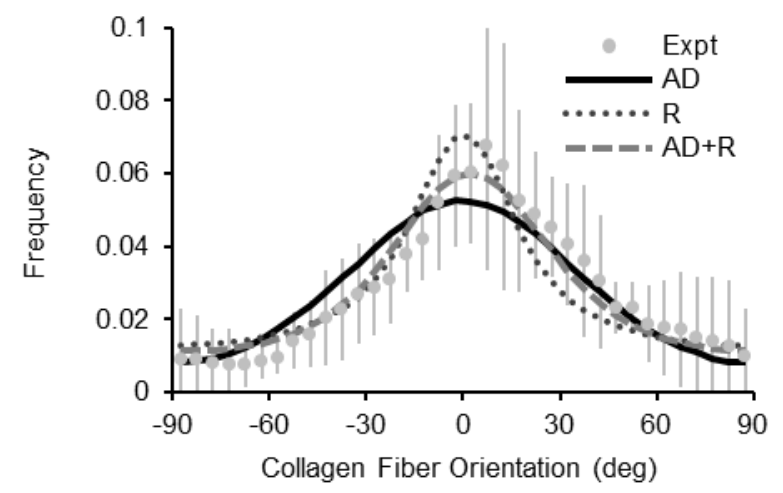

$\mathrm{E}$

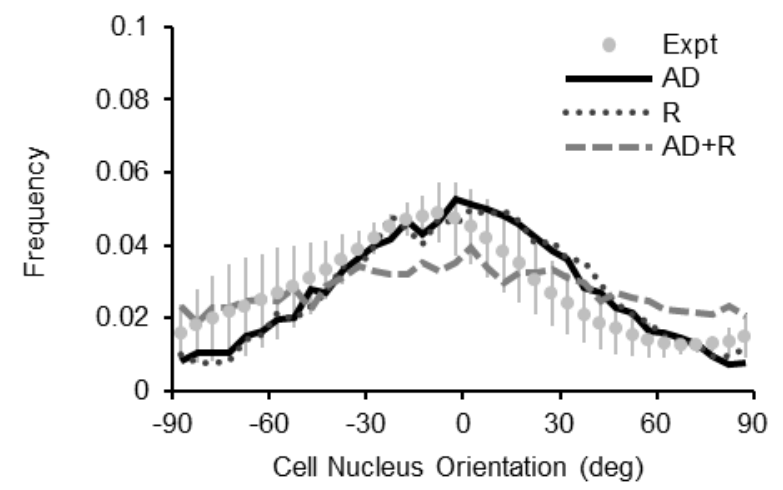

B

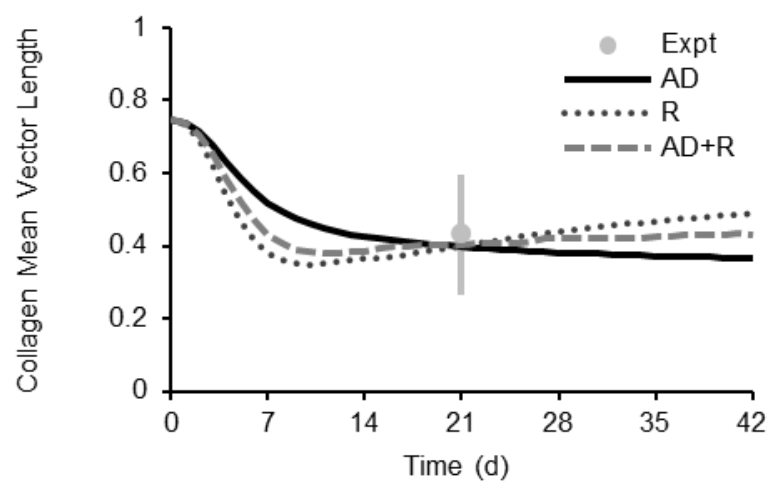

$\mathrm{D}$

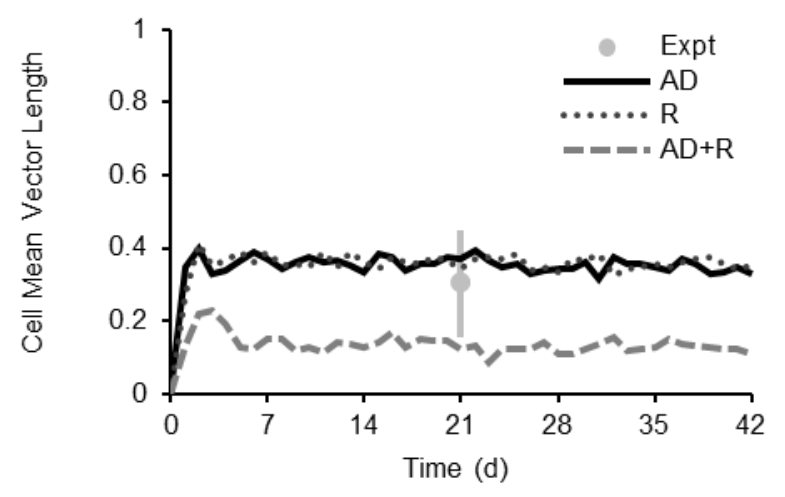

$\mathrm{F}$

Figure 3.5. Collagen remodeling processes. Healing of circular infarcts experiencing uniaxial strain was simulated with models that allowed collagen remodeling via only aligned deposition (AD), only rotation (R), or only selective degradation (SD). For each case, the values of model input parameters were adjusted from the values used in the baseline model from Chapter 2 in an attempt to satisfy all experimental constraints. A. The SD model failed to reproduce the experimental collagen mean vector length at 3 weeks after infarction. B and C. AD, R, and $\mathrm{AD}+\mathrm{R}$ models all predicted similar collagen mean vector length time courses and collagen orientation histograms 3 weeks after infarction that agreed with experimental data. D and E. However, these models predicted different cell mean vector lengths and cell orientation histograms 3 weeks after infarction. The experimentally measured cell orientation histogram agreed with the predictions of the $\mathrm{AD}$ and $\mathrm{R}$ models, but not the prediction of the $\mathrm{AD}+\mathrm{R}$ model. 
A

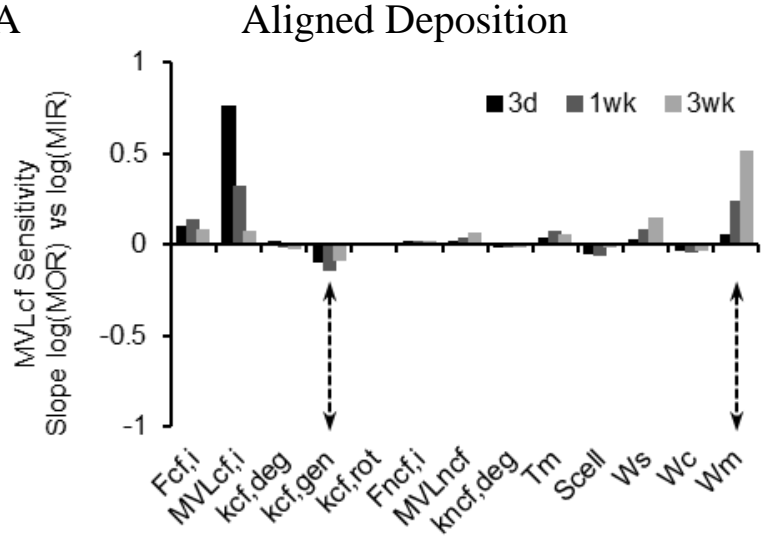

B Rotation

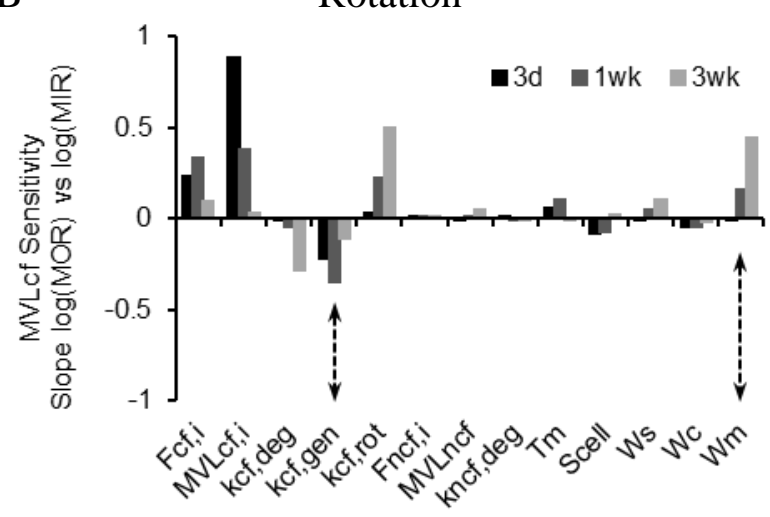

Figure 3.6. Sensitivity of the strength of collagen alignment $\left(\mathrm{MVL}_{\mathrm{cf}}\right)$ to model input parameters. A. $\mathrm{MVL}_{\mathrm{cf}}$ predicted by the $\mathrm{AD}$ model was sensitive to the initial strength of collagen fiber alignment and the mechanical cue weight factor. B. $\mathrm{MVL}_{\mathrm{cf}}$ predicted by the $\mathrm{R}$ model was also sensitive to the initial collagen area fraction, collagen fiber degradation rate coefficient, generation rate coefficient, and rotation rate coefficient. The differences between the two models highlight opportunities for design of experimental interventions that could provide validation data for these models. 
A

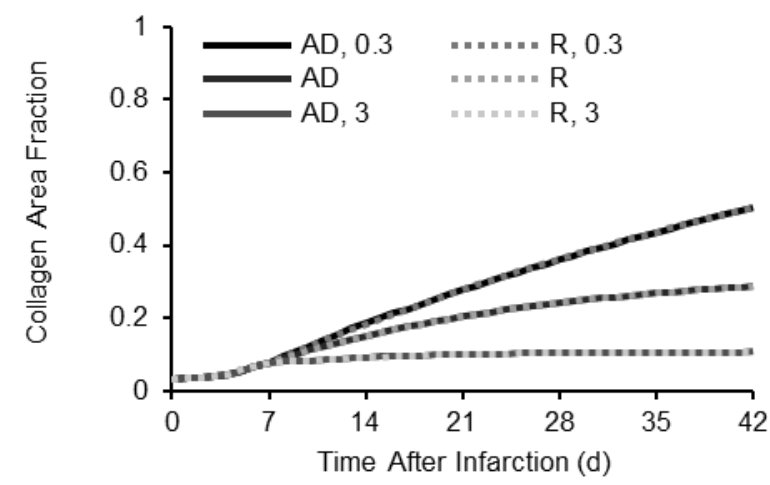

$\mathrm{C}$

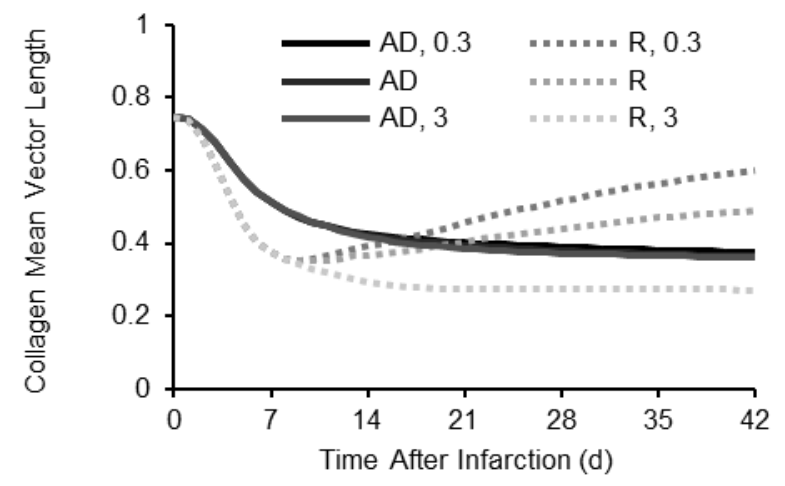

$\mathrm{E}$

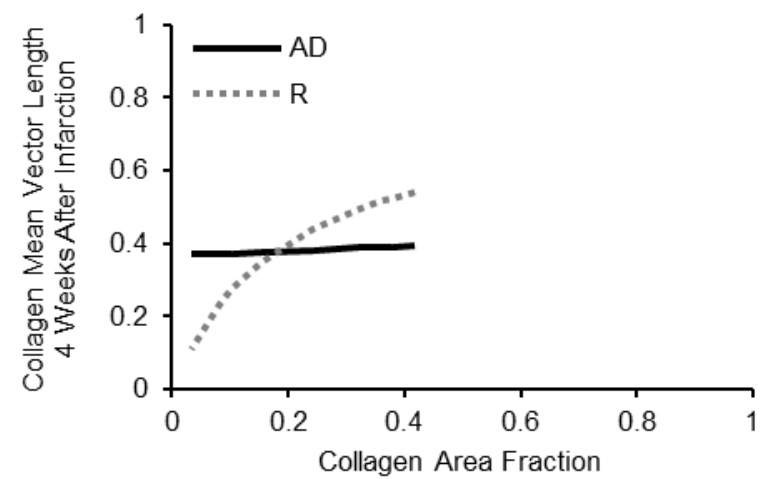

B

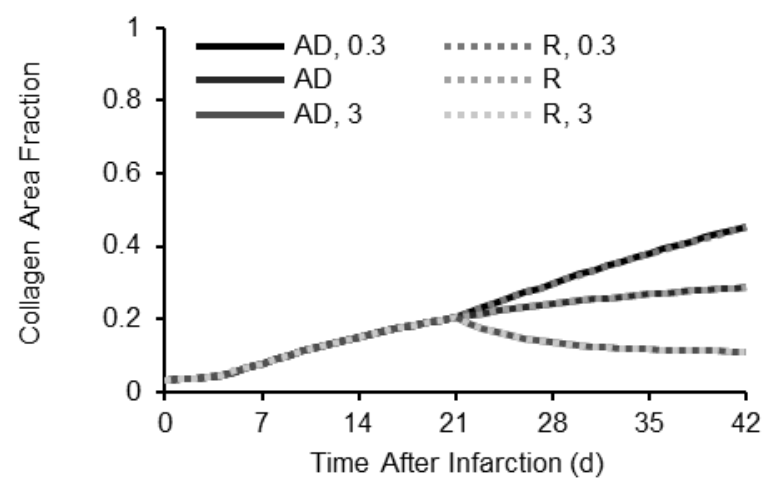

$\mathrm{D}$

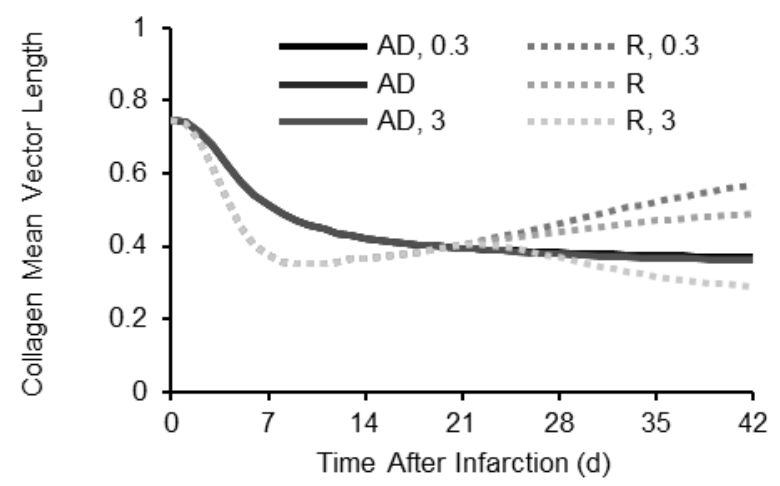

$\mathrm{F}$

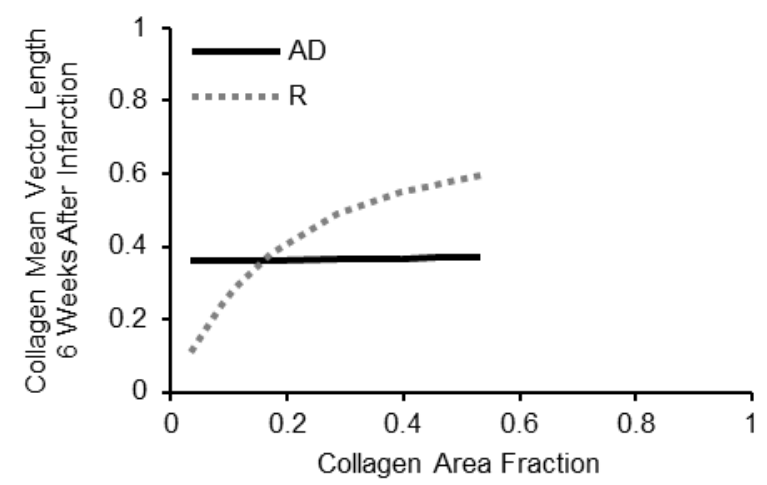

Figure 3.7. Response of $\mathrm{AD}$ and $\mathrm{R}$ models to perturbation of the rate of collagen degradation. $\mathrm{AD}$ and $\mathrm{R}$ models were used to simulate healing of circular infarcts experiencing uniaxial strain. For each case, treatments with agents that up- or down-regulate the rate of collagen degradation were simulated by multiplying the collagen fiber degradation rate coefficient by factors ranging from 1/10 to 10 . Treatment began 1 week (panels A, C, E) or 3 weeks (panels B, D, F) after infarction. A and B. For both models, scaling the collagen fiber degradation rate coefficient up or down (factors of 3 and 0.3 shown) caused the collagen area fraction to approach a lower or higher steady state value. C and D. For the R model, scaling the collagen fiber degradation rate coefficient up or down also caused the strength of collagen fiber alignment to approach a lower or higher steady state value. For the AD model, changing the degradation rate coefficient had no effect on the predicted collagen mean vector length time course. $\mathbf{E}$ and $\mathbf{F}$. Three weeks after intervention, the $\mathrm{R}$ model predicted a positive correlation between collagen mean vector length and collagen area fraction, whereas the AD model predicted no relationship. The correlations were similar whether the interventions began 1 week or 3 weeks after infarction. 
A
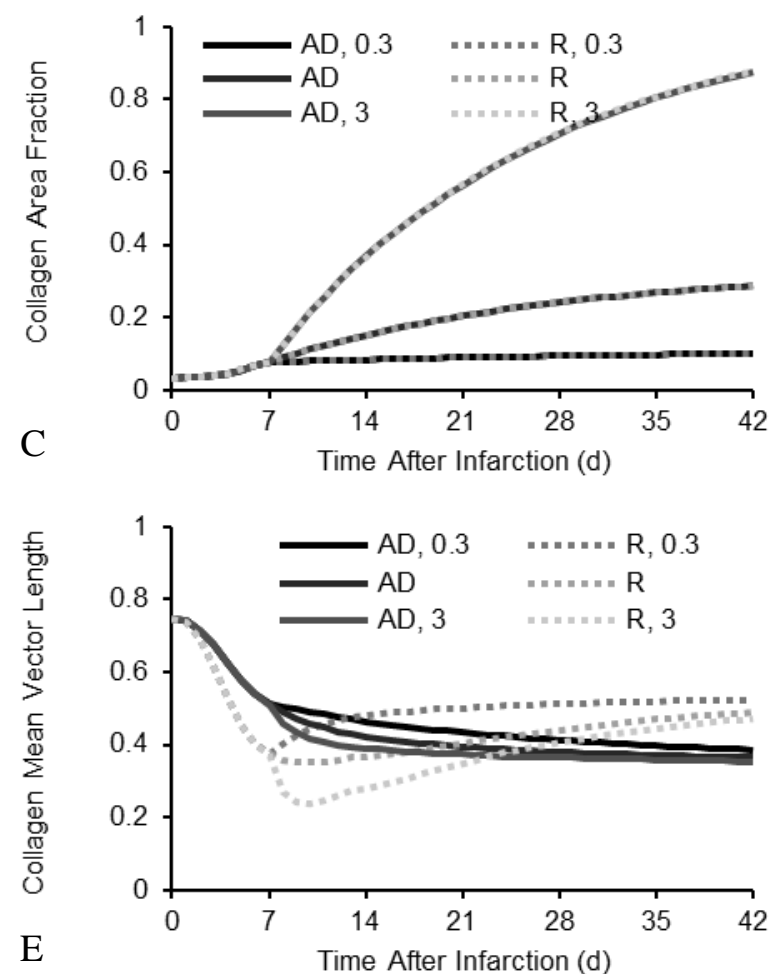

$\mathrm{E}$

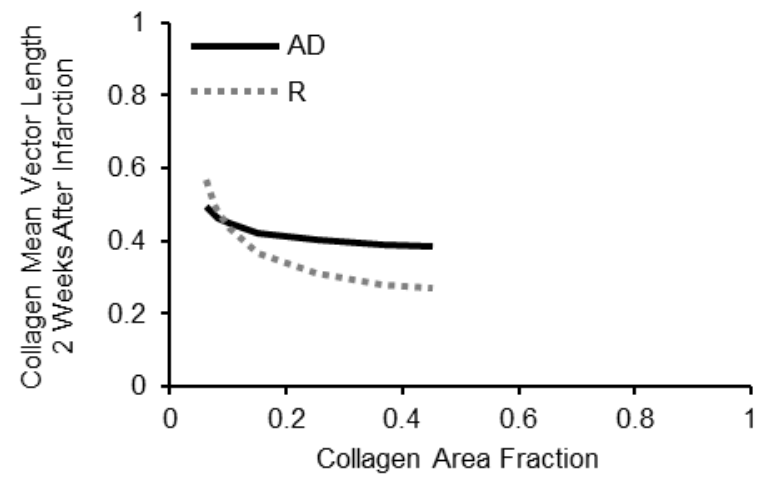

B
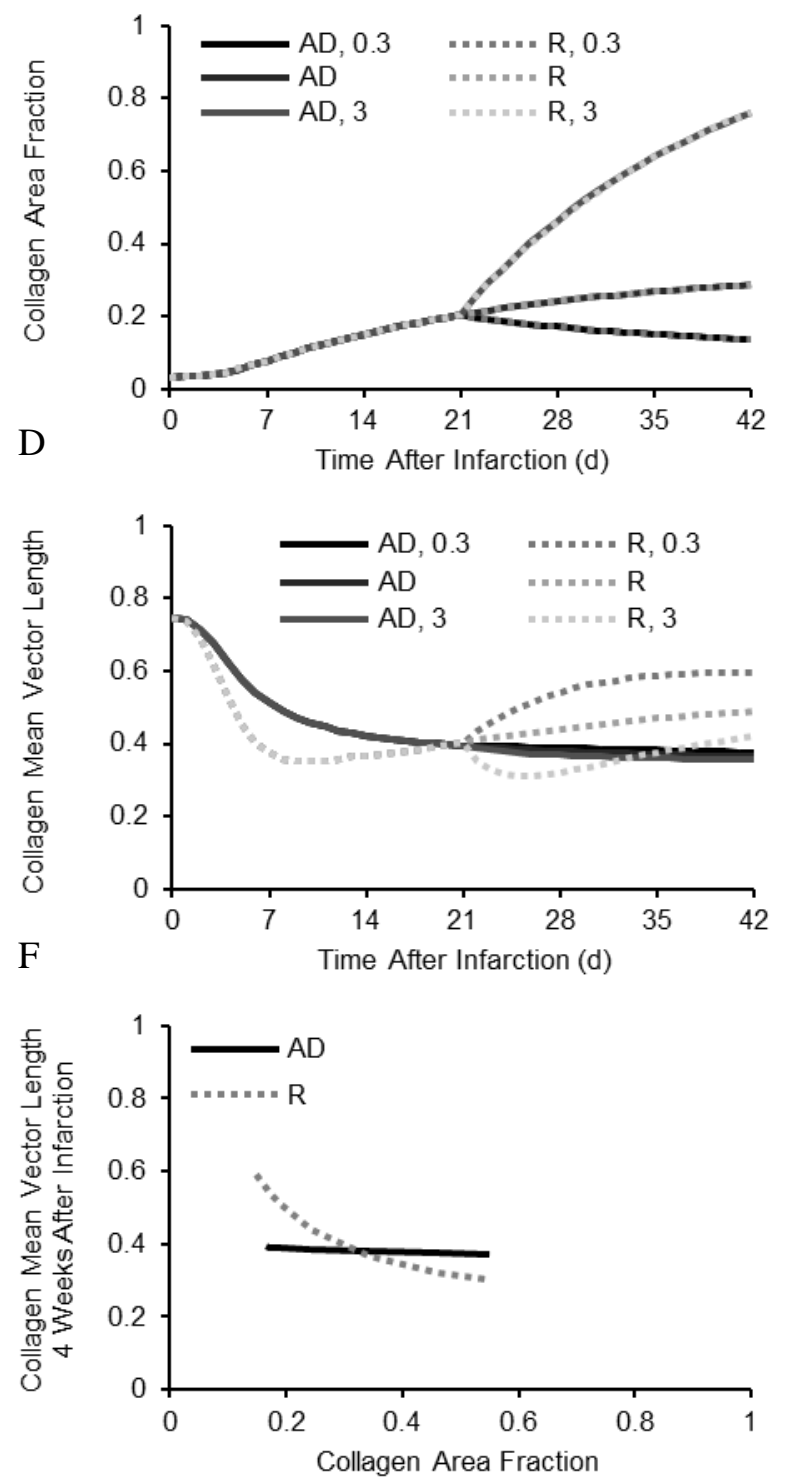

Figure 3.8. Response of $\mathrm{AD}$ and $\mathrm{R}$ models to perturbation of the rate of collagen generation. $\mathrm{AD}$ and $\mathrm{R}$ models were used to simulate healing of circular infarcts experiencing uniaxial strain. For each case, treatments with agents that up- or down-regulate the rate of collagen generation were simulated by multiplying the collagen fiber generation rate coefficient by factors ranging from $1 / 10$ to 4 . Treatment began 1 week (panels A, C, E) or 3 weeks (panels B, D, F) after infarction. A and B. For both models, scaling the collagen fiber generation rate coefficient up or down (factors of 3 and 0.3 shown) caused the collagen area fraction to approach a higher or lower steady state value. C. For both models, scaling the generation rate coefficient up or down beginning 1 week after infarction caused the strength of collagen fiber alignment to transiently decrease or increase relative to the baseline prediction. D. Scaling the generation rate coefficient beginning 3 weeks after infarction caused the strength of collagen fiber alignment to transiently change for the R model but not the AD model. E. Two weeks after infarction (1 week after intervention), both models predicted a negative correlation between collagen mean vector length and collagen area fraction. F. Four weeks after infarction (1 week after intervention), only the R model predicted a negative correlation. 
A

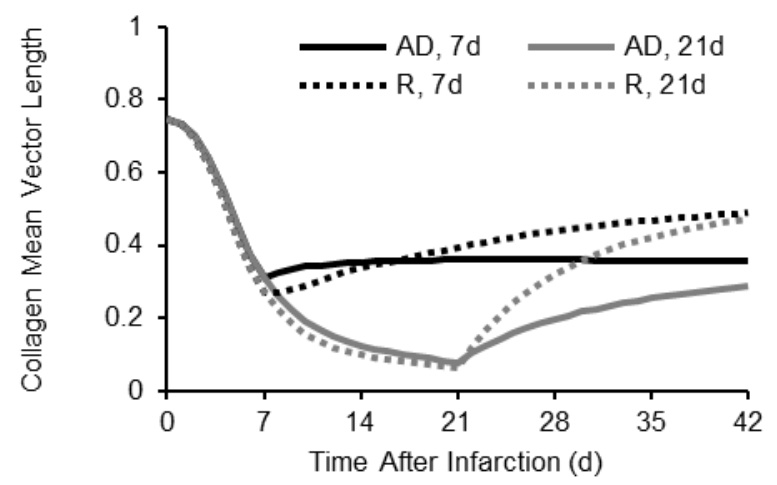

$\mathrm{C}$

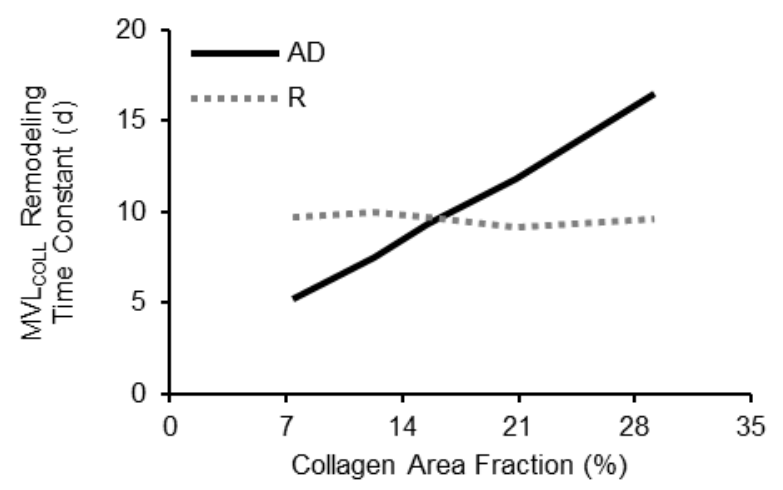

B

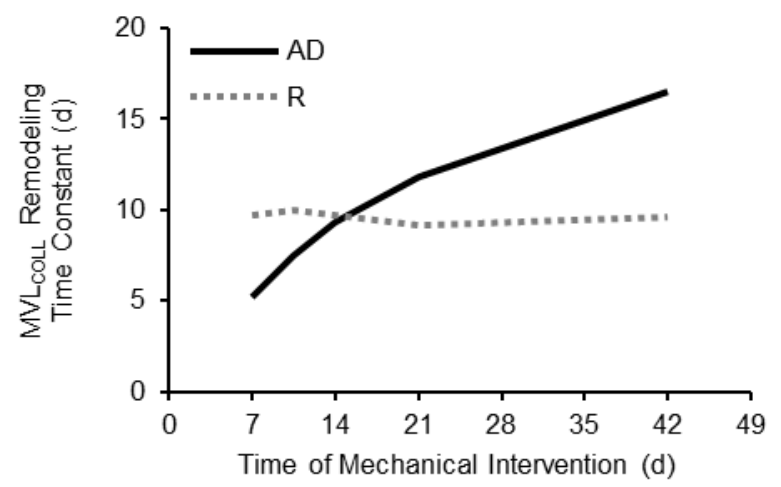

Figure 3.9. Response of $\mathrm{AD}$ and $\mathrm{R}$ models to perturbation of mechanical environment. $\mathrm{AD}$ and $\mathrm{R}$ models were used to simulate healing of circular infarcts that initially experienced equibiaxial strain and then were altered to experience uniaxial strain in the circumferential direction at 7 , $10.5,14,21$, or 42 days after infarction. A. The strength of collagen fiber alignment initially decreased as expected in the absence of a mechanical guidance cue. At the time of the mechanical intervention ( $7 \mathrm{~d}$ and $21 \mathrm{~d}$ shown), the decrease in collagen mean vector length was arrested and the strength of collagen fiber alignment began to increase in response to the mechanical guidance cue. When the mechanical environment was changed at Day 7, the AD model predicted faster remodeling of the collagen matrix than the $\mathrm{R}$ model. When the mechanical environment was changed at Day 21, the $\mathrm{R}$ model predicted faster remodeling of the collagen matrix than the AD model. B. We fit the collagen mean vector length time courses post-intervention to saturating exponential functions in order to determine time constants for the remodeling response. The $\mathrm{AD}$ model predicted slower remodeling of the collagen matrix at later intervention times, whereas the $\mathrm{R}$ model predicted a rate of remodeling independent of the time of mechanical intervention. C. The $\mathrm{AD}$ model predicted a positive correlation between the remodeling time constant and collagen area fraction (at the time of the intervention), whereas the $\mathrm{R}$ model predicted no relationship. This explains why the time constant for the AD model depended on the time of the mechanical intervention. Early after infarction, relatively little collagen was present and the orientation of newly deposited collagen had a large impact on the overall alignment of the collagen matrix. Late after infarction, the amount of newly deposited collagen was small relative to the total amount of collagen and the orientation of newly deposited collagen had little impact on the overall alignment of the collagen matrix. 


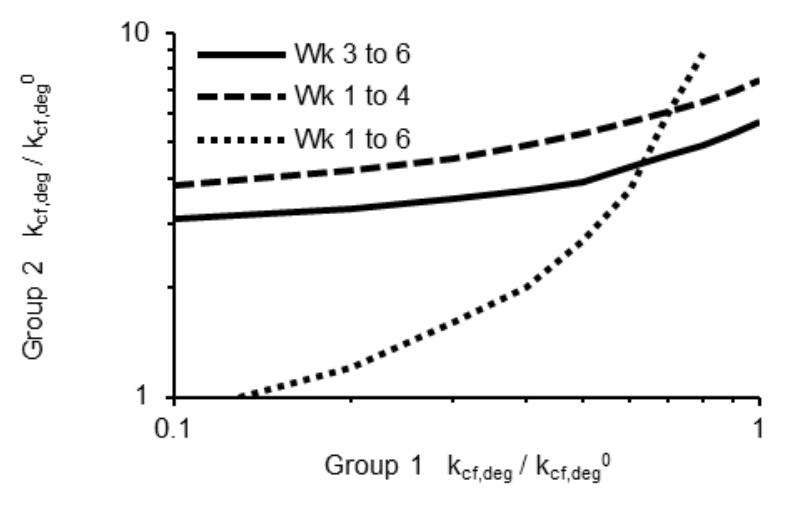

Figure 3.10. Power analysis to determine sample size needed for Experiment 1 (perturbation of the collagen fiber degradation rate). For Experiment 1, we proposed testing if collagen mean vector length differs between two groups (Group 1 - decrease degradation rate, Group 2 increase degradation rate). We used a significance level of 0.05 , power of 0.95 , and standard deviation of 0.234 to calculate the set of perturbation combinations that the $\mathrm{R}$ model predicted would have significantly different collagen mean vector lengths using a sample size of 10 rats per group. We performed this power analysis for intervention times of Week 3 to 6, Week 1 to 4, and Week 1 to 6 . For each intervention time, any prediction for a pair of fold changes that lies on or above the curve could be tested experimentally with a sample size of 10 . 
Table 3.1. Characteristics of cell nuclei in cryoinfarct scar tissue. Six infarcts per group, 2-3 sections per infarct.

\begin{tabular}{|ccc|}
\hline & & \\
\hline Anterior Wall Cryoinfarct & $\underline{\text { Apical Cryoinfarct }}$ \\
Mean Angle (deg) & $-14 \pm 13$ & $39 \pm 124$ \\
Mean Vector Length & $0.30 \pm 0.15$ & $0.03 \pm 0.10$ \\
Circular Standard Deviation (deg) & $44 \pm 9$ & $77 \pm 39$ \\
Nucleus Area ( $\left.\mu \mathrm{m}^{2}\right)$ & $30 \pm 3$ & $34 \pm 2$ \\
Nucleus Aspect Ratio & $2.8 \pm 0.2$ & $2.5 \pm 0.1$ \\
Nucleus Area Fraction & $0.078 \pm 0.008$ & $0.093 \pm 0.004$ \\
Cell Density $\left(\mu \mathrm{m}^{-2}\right)$ & $0.0027 \pm 0.0001$ & $0.0028 \pm 0.0002$ \\
Cell Spacing $(\mu \mathrm{m})$ & $19 \pm 1$ & $19 \pm 1$ \\
\hline
\end{tabular}


Table 3.2. Proposed experiments for determining whether Aligned Deposition or Rotation better explains collagen fiber remodeling during infarct healing.

\begin{tabular}{|c|c|c|c|}
\hline \multicolumn{4}{|c|}{ Intervention } \\
\hline & $\begin{array}{l}1 \\
\text { Change collagen degradation } \\
\text { rate beginning } 1 \text { week after } \\
\text { infarction } \\
\end{array}$ & $\begin{array}{l}2 \\
\text { Change collagen generation } \\
\text { rate beginning } 3 \text { weeks after } \\
\text { infarction } \\
\end{array}$ & $\begin{array}{l}\text { Change mechanics from equibiaxial } \\
\text { to uniaxial at different time points } \\
\text { after infarction }\end{array}$ \\
\hline \multicolumn{4}{|c|}{$\underline{\text { Measurement }}$} \\
\hline & $\begin{array}{c}\mathrm{MVL}_{\mathrm{cf}} \mathrm{Vs} . \mathrm{F}_{\mathrm{cf}} \text { at } 4 \text { weeks after } \\
\text { infarction }\end{array}$ & $\begin{array}{c}\mathrm{MVL}_{\mathrm{cf}} \text { vs. } \mathrm{F}_{\mathrm{cf}} \text { at } 4 \text { weeks after } \\
\text { infarction }\end{array}$ & $\begin{array}{l}\mathrm{MVL}_{\mathrm{cf}} \text { remodeling time constant vs. } \\
\text { Time of intervention (or } \mathrm{F}_{\mathrm{cf}} \text { ) }\end{array}$ \\
\hline Model & & Predicted Correlation & \\
\hline $\begin{array}{c}\mathrm{AD} \\
\mathrm{R}\end{array}$ & $\begin{array}{c}\text { None } \\
\text { Positive }\end{array}$ & $\begin{array}{c}\text { None } \\
\text { Negative }\end{array}$ & $\begin{array}{l}\text { Positive } \\
\text { None }\end{array}$ \\
\hline
\end{tabular}




\section{Appendix 3.A. Summary of selective degradation studies}

Bhole et al. (2009) and Flynn et al. (2010) used micropipettes to apply tensile strain to a collagen gel, exposed the gel to bacterial collagenase or MMP-8, and used DIC microscopy to quantify the disappearance of collagen fibers over time. Both studies observed that fibers subjected to tensile strain decayed approximately $25 \%$ slower than unstrained fibers. In these studies the level of strain was not quantified.

Camp et al. (2011) used collagen monomers to link magnetic micro-beads to a substrate, exposed the substrate to collagenase, applied a magnetic force to the beads in order to stretch the collagen linker, and quantified the release of beads from the substrate over time. This study observed that collagen monomers subjected to approximately $4 \mathrm{pN}$ of force were cleaved at $1 / 10^{\text {th }}$ the rate of unloaded monomers. No further decrease in the cleavage rate was observed at $9 \mathrm{pN}$ of force. A wormlike chain model was used to calculate monomer strains of $95 \%$ and $107 \%$ for 4 and $9 \mathrm{pN}$ of force, respectively, which were located at the "heel" of the stress-strain curve. It is uncertain if this finding can be extended to collagen fibers or collagenous tissues, such that loading these materials to the heel of their stress-strain curve would cause a 10-fold decrease in the collagen degradation rate.

Wyatt et al. (2009) applied tensile strain to rat tail tendon fascicles, waited for stress relaxation, exposed the fascicles to collagenase, and quantified the additional stress relaxation over time due to collagen degradation. This study observed that increasing strain from zero through the heel of 
the stress-strain curve of the fascicles caused the collagen degradation rate to decrease somewhere from 5 to 10 -fold.

Huang and Yannas (1977) applied tensile strain to collagen tapes and quantified degradationinduced stress relaxation over time as the difference between stress relaxation curves for experiments with and without collagenase. This study acquired sufficient data to observe the collagen degradation rate decrease approximately 5-fold as strain increased beyond the heel of the stress-strain curve of the material, then the degradation rate began to increase as strain approached the failure regime of the material.

Zareian et al. (2010) applied tensile stress to bovine cornea, waited for the material to creep, exposed the cornea to collagenase, and quantified the additional creep over time due to collagen degradation. This study reported a 3 or 4 -fold decrease in the rate of collagen degradation when tensile strain was increased from $4 \%$ to $6 \%$. It is unknown where these strains lay on the material's stress-strain curve.

Ellsmere et al. (1999) applied tensile stress to bovine pericardium, exposed the pericardium to collagenase, quantified the creep response until failure, and measured the final amount of solubilized collagen. This study observed that applied loads that caused instantaneous strain of about $12 \%$ caused material failure approximately $50 \%$ faster than loads that caused instantaneous strain of only $2 \%$. A strain of $12 \%$ was estimated to be approaching the failure regime of the material's stress-strain curve. 
Overall, the studies by Bhole, Flynn, Camp, Wyatt, and Zareian suggest that increasing strain through the heel of a collagenous material's stress-strain curve causes the rate of enzymatic degradation of collagen to decrease roughly 5 to 10 -fold. The study by Ellsmere suggests that higher strains cause the degradation rate to increase. The study by Huang and Yannas captured both of these effects, showing the collagen degradation rate to decrease, reach a minimum, and then increase with increasing strain (Fig. 3.1). 


\section{APPENDIX 3.B. Calculation of selective degradation rate function}

The collagen fiber degradation rate coefficient as a function of fiber orientation for use in the selective degradation model was derived as follows. The first order differential equation governing the angular density of collagen fibers at any orientation $\theta$ was defined as

$\frac{d \rho_{c f}}{d t}=\frac{k_{c f, g e n}}{2 \pi}-k_{c f, \operatorname{deg}}(\theta) \rho_{c f}$

$\rho_{c f}-$ angular density of collagen fibers (area fraction / radian), $k_{c f, g e n}-$ bulk collagen fiber generation rate coefficient (area fraction $/ \mathrm{hr}$ ), $k_{c f, d e g}(\theta)$ - angular collagen fiber degradation rate coefficient $\left(\mathrm{hr}^{-1} \operatorname{radian}^{-1}\right), t-$ time $(\mathrm{hr}), \theta$ - fiber orientation (radian)

which has the solution

$\rho_{c f}(t, \theta)=\left[\rho_{c f}(0, \theta)-\rho_{c f}(\infty, \theta)\right] e^{-k_{c f, d e g}(\theta)\left(t-t_{0}\right)}+\rho_{c f}(\infty, \theta)$

$\rho_{c f}(0, \theta)$ - angular density of collagen fibers at $t_{0}, \rho_{c f}(\infty, \theta)$ - angular density of collagen fibers at steady state

where

$\rho_{c f}(\infty, \theta)=\frac{k_{c f, g e n} /(2 \pi)}{k_{c f, \operatorname{deg}}(\theta)}$ 
The collagen fiber orientation distribution was assumed to have the shape of two wrapped normal distributions offset by 180 degrees (because it is only possible to define an axis along which fibers are oriented, not a single orientation), which implies that the angular density of collagen fibers takes the form

$\rho_{c f}(t, \theta)=F_{c f}(t) \frac{1}{2}\left[\phi_{w n}(\theta: \sigma(t), 0)+\phi_{w n}(\theta: \sigma(t), \pi)\right]$

$\phi_{w n}(\theta: \sigma, \bar{\theta})$ - wrapped normal probability density function, $\sigma$ - circular standard deviation of wrapped normal distribution, $\bar{\theta}-$ mean angle of wrapped normal distribution, $F_{c f}-$ collagen area fraction

and also implies that

$F_{c f}(t)=\int_{-\pi}^{\pi} \rho_{c f}(t, \theta) d \theta$

By substitution, Equation 2 can be written as:

$$
\begin{aligned}
& F_{c f}(t) \frac{1}{2}\left[\phi_{w n}(\theta: \sigma(t), 0)+\phi_{w n}(\theta: \sigma(t), \pi)\right]= \\
& \left\{F_{c f}(0) \frac{1}{2}\left[\phi_{w n}(\theta: \sigma(0), 0)+\phi_{w n}(\theta: \sigma(0), \pi)\right]-\frac{k_{c f, g e n} /(2 \pi)}{k_{c f, d e g}(\theta)}\right\} e^{-k_{c f, d e g}(\theta)\left(t-t_{0}\right)}+\frac{k_{c f, g e n} /(2 \pi)}{k_{c f, d e g}(\theta)}
\end{aligned}
$$

(Eqn. 3.6) 
Finally, the collagen fiber degradation rate coefficient was assumed to be 10-fold lower for fibers oriented along the axis of stretch relative to fibers oriented perpendicular to the axis of stretch:

$k_{c f, \operatorname{deg}}(0)=k_{c f, \operatorname{deg}}(\pi)=0.1 k_{c f, \operatorname{deg}}\left( \pm \frac{\pi}{2}\right)$

The known variables in this problem were $k_{c f, g e n}, \sigma(0), t_{0}$, and $F_{c f}(0)$. The unknown variables were $k_{c f, \operatorname{deg}}(\theta)$ and $\sigma(t)$. The time $t$ was chosen to be 21 days, which determined $F_{c f}(t)$. In order to solve for $k_{c f, \operatorname{deg}}(\theta)$, we guessed $\sigma(t)$ and used Equation 6 to solve for $k_{c f, \text { deg }}(0)$ and $k_{c f, \operatorname{deg}}(\pi)$, then checked if Equation 7 was satisfied. If satisfied, then Equation 6 was used to calculate $k_{c f, \operatorname{deg}}(\theta)$ for all $\theta$ from $-\pi$ to $\pi$. Otherwise, we guessed a new $\sigma(t)$ and repeated the check. 


\subsection{REFERENCES}

Bayer ML, Yeung C-YC, Kadler KE, Qvortrup K, Baar K, Svensson RB, Magnusson SP, Krogsgaard M, Koch M \& Kjaer M (2010). The initiation of embryonic-like collagen fibrillogenesis by adult human tendon fibroblasts when cultured under tension. Biomaterials 31, 4889-4897.

Bhole AP, Flynn BP, Liles M, Saeidi N, Dimarzio CA \& Ruberti JW (2009). Mechanical strain enhances survivability of collagen micronetworks in the presence of collagenase: implications for load-bearing matrix growth and stability. Philosophical Transactions of the Royal Society A: Mathematical, Physical and Engineering Sciences 367, 3339-3362.

Camp RJ, Liles M, Beale J, Saeidi N, Flynn BP, Moore E, Murthy SK \& Ruberti JW (2011). Molecular mechanochemistry: low force switch slows enzymatic cleavage of human type I collagen monomer. Journal of the American Chemical Society 133, 4073-4078.

Canty EG, Lu Y, Meadows RS, Shaw MK, Holmes DF \& Kadler KE (2004). Coalignment of plasma membrane channels and protrusions (fibripositors) specifies the parallelism of tendon. The Journal of Cell Biology 165, 553-563.

Champy C \& Carleton H (1921). Observations on the shape of the nucleus and its determination. Quarterly Journal of Microscopical Science 65, 589-625.

Creemers EE, Cleutjens JP, Smits JF \& Daemen MJ (2001). Matrix metalloproteinase inhibition after myocardial infarction: a new approach to prevent heart failure? Circulation Research 89, 201-210.

Ellsmere JC, Khanna RA \& Lee JM (1999). Mechanical loading of bovine pericardium accelerates enzymatic degradation. Biomaterials 20, 1143-1150.

Flynn BP, Bhole AP, Saeidi N, Liles M, DiMarzio CA \& Ruberti JW (2010). Mechanical strain stabilizes reconstituted collagen fibrils against enzymatic degradation by mammalian collagenase matrix metalloproteinase 8 (MMP-8). PLoS ONE 5, e12337.

Fomovsky GM, Clark SA, Parker KM, Ailawadi G \& Holmes JW (2012a). Anisotropic reinforcement of acute anteroapical infarcts improves pump function. Circulation Heart Failure 5, 515-522.

Fomovsky GM \& Holmes JW (2010). Evolution of scar structure, mechanics, and ventricular function after myocardial infarction in the rat. American Journal of Physiology: Heart and Circulatory Physiology 298, H221-H228.

Fomovsky GM, Rouillard AD \& Holmes JW (2012b). Regional mechanics determine collagen fiber structure in healing myocardial infarcts. Journal of Molecular and Cellular Cardiology 52, 1083-1090. 
Holmes JW, Nuñez JA \& Covell JW (1997). Functional implications of myocardial scar structure. American Journal of Physiology: Heart and Circulatory Physiology 272, H2123$\mathrm{H} 2130$.

Huang C \& Yannas I (1977). Mechanochemical studies of enzymatic degradation of insoluble collagen fibers. Journal of Biomedical Materials Research 8, 137-154.

Jugdutt BI (2008a). Aging and remodeling during healing of the wounded heart: current therapies and novel drug targets. Current Drug Targets 9, 325-344.

Jugdutt BI (2008b). Pleiotropic effects of cardiac drugs on healing post-MI. Heart Failure Reviews 13, 439-452.

Kadler KE, Hill A \& Canty-Laird EG (2008). Collagen fibrillogenesis: fibronectin, integrins, and minor collagens as organizers and nucleators. Current Opinion in Cell Biology 20, 495-501.

Kapacee Z, Richardson SH, Lu Y, Starborg T, Holmes DF, Baar K \& Kadler KE (2008). Tension is required for fibripositor formation. Matrix Biology 27, 371-375.

Lee EJ, Holmes JW \& Costa KD (2008). Remodeling of engineered tissue anisotropy in response to altered loading conditions. Annals of Biomedical Engineering 36, 1322-1334.

Loftis MJ, Sexton D \& Carver W (2003). Effects of collagen density on cardiac fibroblast behavior and gene expression. Journal of Cellular Physiology 196, 504-511.

McKee CT, Raghunathan VK, Nealey PF, Russell P \& Murphy CJ (2011). Topographic modulation of the orientation and shape of cell nuclei and their influence on the measured elastic modulus of epithelial cells. Biophysical Journal 101, 2139-2146.

Omens JH, MacKenna DA \& McCulloch AD (1993). Measurement of strain and analysis of stress in resting rat left ventricular myocardium. Journal of Biomechanics 26, 665-676.

Pang Y, Wang X, Lee D \& Greisler HP (2011). Dynamic quantitative visualization of single cell alignment and migration and matrix remodeling in 3-D collagen hydrogels under mechanical force. Biomaterials 32, 3776-3783.

Petroll WM \& Ma L (2003). Direct, dynamic assessment of cell-matrix interactions inside fibrillar collagen lattices. Cell Motility and the Cytoskeleton 55, 254-264.

Petroll WM, Ma L \& Jester J V (2003). Direct correlation of collagen matrix deformation with focal adhesion dynamics in living corneal fibroblasts. Journal of Cell Science 116, 14811491.

Rane AA \& Christman KL (2011). Biomaterials for the treatment of myocardial infarction: a 5year update. Journal of the American College of Cardiology 58, 2615-2629. 
Rape AD, Guo W-H \& Wang Y-L (2011). The regulation of traction force in relation to cell shape and focal adhesions. Biomaterials 32, 2043-2051.

Spinale FG (2007). Myocardial matrix remodeling and the matrix metalloproteinases : influence on cardiac form and function. Physiological Reviews 87, 1285-1342.

Thomopoulos S, Fomovsky GM \& Holmes JW (2005). The development of structural and mechanical anisotropy in fibroblast populated collagen gels. Journal of Biomechanical Engineering 127, 742-750.

Vanhoutte D, Schellings M, Pinto Y \& Heymans S (2006). Relevance of matrix metalloproteinases and their inhibitors after myocardial infarction: a temporal and spatial window. Cardiovascular Research 69, 604-613.

Wang JH-C, Jia F, Gilbert TW \& Woo SL-Y (2003). Cell orientation determines the alignment of cell-produced collagenous matrix. Journal of Biomechanics 36, 97-102.

Weisman HF, Bush DE, Mannisi JA \& Bulkley BH (1985). Global cardiac remodeling after acute myocardial infarction: a study in the rat model. Journal of the American College of Cardiology 5, 1355-1362.

Wyatt KE-K, Bourne JW \& Torzilli PA (2009). Deformation-dependent enzyme mechanokinetic cleavage of type I collagen. Journal of Biomechanical Engineering 131, 051004.

Zareian R, Church KP, Saeidi N, Flynn BP, Beale JW \& Ruberti JW (2010). Probing collagen/enzyme mechanochemistry in native tissue with dynamic, enzyme-induced creep. Langmuir 26, 9917-9926. 


\section{CHAPTER 4: Empirical Approach to Predicting Cell Alignment and Biased Migration Driven by Mechanical, Structural, and Simultaneous Guidance Cues}

\subsection{INTRODUCTION}

After myocardial infarction, fibroblasts infiltrate the damaged tissue, deposit collagen, and remodel collagen (Souders et al., 2009). Our analysis from Chapter 3 suggested that collagen fiber alignment in infarct scar follows from the alignment of fibroblasts, which deposit collagen fibers along the axis of cell alignment or generate traction forces that reorient nearby collagen fibers (Fig. 3.5). Consequently, understanding how environmental guidance cues determine fibroblast alignment is critical to understanding the regulation of collagen fiber alignment.

In vitro, fibroblasts and other cells have been shown to align along the axis of uniaxial stretch (in 3D) (Henshaw et al., 2006; Lee et al., 2008; Pang et al., 2011), perpendicular to the axis of uniaxial stretch (in 2D) (Neidlinger-Wilke et al., 2001), along aligned fibers or micro-ridges (Sun et al., 2010), and along gradients of substrate stiffness (Lo et al., 2000), adhesion ligand (Smith et al., 2004), chemokines (Knapp et al., 1999; Wang, 2009; Melvin et al., 2011) and electric field (Zhao, 2009). Our analysis from Chapter 2 suggested that alignment of fibroblasts along a mechanical guidance cue was necessary to explain the collagen fiber alignment we observed in rat cryoinfarct scars (Fig. 2.11). Furthermore, we found that interaction of the mechanical guidance cue-uniaxial stretch along the circumferential axis $\left(0^{\circ}\right)$ of the left ventricle — with a structural guidance cue — initial fiber structure of normal myocardium, which has depth-dependent orientation varying from $-60^{\circ}$ at the epicardium to $+60^{\circ}$ at the 
endocardium - could explain the depth-dependent variation of collagen fiber angle and collagen mean vector length observed in 3 week old pig ligation infarct scars (Fig. 2.10). Consequently, understanding how mechanical, structural, and simultaneous mechanical and structural guidance cues determine fibroblast alignment is of particular importance in the context of infarct healing.

Prior studies have modeled the influence of guidance cues on cell alignment by representing guidance cues as vectors, computing the weighted sum of these vectors, and defining the cell orientation as the orientation of the resultant vector (McDougall et al., 2006; Groh \& Wagner, 2011). In our agent-based model, we modified this approach by using the resultant vector to define a probability distribution of cell orientations, in order to account for observations that guidance cues bias cell orientations but rarely dictate orientations absolutely. This approach worked well for predicting the cell and collagen fiber alignment observed in healing infarcts, but some of the parameter values and equations we used to compute and combine the guidance cue vectors were not firmly grounded in experimental evidence. For example, in order to calculate strengths of cell alignment for individual guidance cues we simply divided the magnitudes of the guidance cues by normalization factors. Also, we inferred the ratio of the mechanical and structural guidance cue weight factors $\left(W_{m} / W_{s}\right)$ from the cryoinfarct data, rather than from external data. These limitations reduce our confidence in applying our approach to predict fibroblast orientations in novel environments.

We sought a stronger empirical foundation for our approach to predicting fibroblast alignment and migration in response to environmental guidance cues. We tested and refined our approach using experimental data gathered from published, in vitro studies of the alignment of cells in 
response to mechanical (cyclic uniaxial stretch applied to elastic substrates), structural (microridged substrates), and simultaneous mechanical and structural guidance cues (cyclic uniaxial stretch applied to elastic, micro-ridged substrates). We used the literature data to formulate equations and fit for parameters that could predict cell orientation distributions from measurable characteristics of mechanical and structural guidance cues, such as strain amplitudes and microridge (or fiber) size. We then revisited the data from Dickinson et. al. (1994), who measured the migration of fibroblasts in collagen gels with aligned fibers, in order to test if we could extend our approach to predicting cell orientation distributions to also predict how fibroblast migration is biased by guidance cues. Finally, we updated our agent-based model with the new equations and parameter values in order to assess whether the in vitro data contradicts the conclusions we drew previously about the roles of structural and mechanical guidance cues during infarct healing.

We found that the literature data generally supported our approach to representing guidance cues as vectors, combining guidance cues as a weighted vector sum, relating the resultant vector to a cell orientation probability distribution, and randomly sampling this probability distribution to periodically update the migration trajectories of simulated cells. The data also specifically supported the predictions of our agent-based model of infarct healing. Overall, we present the foundation of an approach to predicting the orientation and migration of fibroblasts in response to environmental guidance cues, which can be employed in multi-scale, cell-based models. We conclude by highlighting knowledge gaps and propose experiments that would address them. 


\subsection{METHODS}

The aim of this study was to develop an evidence-based approach to predicting the orientation and migration of fibroblasts in response to environmental guidance cues. We chose to limit our investigation to the alignment of cells in response to mechanical, structural, or simultaneous mechanical and structural guidance cues. We aggregated cell alignment data from published studies, formulated equations that captured the general features of the data, and fit for equation parameters. We then compared the cell alignment predicted by the new equations to the cell alignment predicted by the approximated equations that we used for our infarct healing simulations. Finally, we compared the predictions of the agent-based model of infarct healing using the new equations and parameter values to the predictions of the prior version of the model.

\section{Data Collection and Processing}

\section{Cell Alignment}

Our approach was to search the literature for studies that measured the alignment of cells in response to mechanical, structural, or simultaneous mechanical and structural guidance cues. Studies that reported sufficiently quantitative data were almost exclusively performed with cells plated on 2-dimensional (2D) substrates. Cells were either exposed to cyclic uniaxial stretch (Wang \& Grood, 2000; Neidlinger-Wilke et al., 2001; Loesberg et al., 2005; Kaunas \& Hsu, 2009) or plated on substrates etched with arrays of parallel micro-ridges (Clark et al., 1990; 
Sutherland et al., 2005; Loesberg et al., 2007; Kim et al., 2009; Pot et al., 2010; Sun et al., 2010). A handful of studies measured the alignment of cells plated on micro-ridged substrates and subjected to cyclic uniaxial stretch (Wang \& Grood, 2000; Wang et al., 2004, 2005; Loesberg et al., 2005), but only one of these studies examined a range of conditions sufficient for useful analysis (Prodanov et al., 2010). Due to the scarcity of studies that reported sufficiently quantitative data, we included data for any cell type. From our literature search, we assembled measurements of the orientation and strength of alignment of cells as a function of 1 . amplitude of cyclic uniaxial strain applied to $2 \mathrm{D}$ substrates at a frequency of $1 \mathrm{~Hz}, 2$. height and pitch (spacing) of ridges on micro-patterned substrates, and 3. amplitude of cyclic uniaxial strain applied to micro-ridged substrates.

These studies reported a variety of alignment metrics: cell orientation histograms, box and whisker plots, percentage of cells oriented within 10 degrees of the alignment axis, arithmetic standard deviation (only valid if orientations are measured as the angle away from the alignment axis, ranging from -90 to 90 degrees), and arithmetic mean angle (only valid if orientations are measured as the absolute value of the angle away from the alignment axis, ranging from 0 to 90 degrees). In order to interpret these data as a whole, we needed to convert them to a common metric. Because mechanical and structural guidance cues are bidirectional (they cause cells to orient along an axis without preferring either of the two antiparallel directions along that axis), we assumed for each experiment that the underlying cell orientation distribution could be represented as the average of two identical wrapped normal probability density functions offset by 180 degrees (Fig. 4.1): 
$\varphi(\theta)=\frac{1}{2}\left[\varphi_{w n}(\theta: \rho, \bar{\theta})+\varphi_{w n}(\theta: \rho, \bar{\theta}+\pi)\right]$

$\varphi_{w n}(\theta: \rho, \bar{\theta})=\frac{1}{\sigma \sqrt{2 \pi}} \sum_{k=-\infty}^{\infty} e^{-(\theta-\bar{\theta}+2 \pi k)^{2} /(2 \sigma)^{2}}$

$\sigma^{2}=-2 \ln \rho$

$\varphi-$ cell orientation probability density function, $\theta-$ cell orientation, $\varphi_{w n}-$ wrapped normal probability density function, $\rho$ - mean vector length of the wrapped normal probability density function, $\bar{\theta}-$ mean angle of the wrapped normal probability density function, $\sigma$ - circular standard deviation of the wrapped normal probability density function

The wrapped normal distribution is defined by a mean angle $(\bar{\theta})$ and mean vector length $(\rho)$, which we used as common metrics of the orientation and strength of alignment of cells (mean vector length can vary from 0 to 1 , where 0 indicates no alignment and 1 indicates perfect alignment). By using the mean angle and mean vector length of a wrapped normal distribution to describe the orientation and strength of alignment of cells, we can also define a cell orientation vector:

$\vec{c}=\rho \cos (\bar{\theta}) \vec{e}_{x}+\rho \sin (\bar{\theta}) \vec{e}_{y}$

$\vec{c}-$ cell orientation vector, $\rho$ - mean vector length of the wrapped normal probability density function describing the cell orientation distribution, $\bar{\theta}-$ mean angle of the wrapped normal probability density function describing the cell orientation distribution

The concept of a cell orientation vector will be useful later for the problem of computing cell alignment in response to simultaneous guidance cues. 
For each experiment, we solved for the cell orientation vector consistent with the experimental data. That is, we iteratively guessed a mean angle and mean vector length, calculated the prescribed cell orientation distribution, computed the alignment metric reported in the study, and checked if it matched the experimental data.

For the mechanical cue data set, we obtained mean angles $\left(\bar{\theta}_{m}\right)$ and mean vector lengths $\left(\rho_{m}\right)$ for a range of strain anisotropies $(\Delta \varepsilon)$, which we defined as the difference between the principal strains:

$\Delta \varepsilon=\varepsilon_{1}-\varepsilon_{2}$

$\Delta \varepsilon-$ strain anisotropy, $\varepsilon_{i}-$ principal strain

For the structural cue data set, we obtained mean angles $\left(\bar{\theta}_{s}\right)$ and mean vector lengths $\left(\rho_{s}\right)$ for a range of ridge heights $(H)$ and pitches $(P)$.

For the third data set, which involved simultaneous mechanical and structural guidance cues that acted perpendicular to each other, we obtained mean angles and mean vector lengths for a range of strain anisotropies, where cyclic uniaxial strain was applied to smooth substrates and applied to ridged substrates, including zero strain controls for both substrates. Thus, given alignment data for a mechanical cue alone, structural cue alone, and then simultaneous cues, we were able to ask how the two cues must interact in order to yield the alignment observed when they act simultaneously. 


\section{Cell Migration}

Dickinson et al. (1994) created collagen gels with varying strengths of collagen fiber alignment and measured the migration of fibroblasts in these gels. Previously, we used the migration data to estimate parameters in our agent-based model (migration speed, model time step, persistence and structural cue weight factors), but Dickinson et al. also measured the strength of alignment of fibroblasts in their collagen gels, which we had not taken into account at the time. From the study by Dickinson et al., we obtained random migration coefficients for motion along the fiber and cross-fiber axes of the collagen gels and the mean vector lengths of the cell orientation distributions. We used this data to formulate an approach to predicting fibroblast migration tracks from the cell orientation distribution expected for a given guidance cue.

\section{Modeling}

\section{Cell Alignment}

We sought equations that could predict the orientation (mean angle) and strength of cell alignment (mean vector length) in response to mechanical, structural, or simultaneous mechanical and structural guidance cues. For the mechanical cue data set, we sought an equation for mean vector length $\left(\rho_{m}\right)$ as a function of strain anisotropy $(\Delta \varepsilon)$. For the structural cue data set, we sought an equation for mean vector length $\left(\rho_{s}\right)$ as a function of ridge height $(H)$ and pitch

$(P)$. For the simultaneous cues data set, we sought an equation for mean angle $\left(\bar{\theta}_{r}\right)$ and mean vector length $\left(\rho_{r}\right)$ of the resultant or apparent guidance cue as a function of the mean angles $\left(\bar{\theta}_{m}\right.$ 
and $\left.\bar{\theta}_{s}\right)$ and mean vector lengths $\left(\rho_{m}\right.$ and $\left.\rho_{s}\right)$ of the individual mechanical and structural guidance cues.

Because the strength of cell alignment is bounded, we expected the dependence of strength of cell alignment $(\rho)$ on guidance cue magnitude $(M)$ to take the form of a saturation function. We decided to try Hill-like functions of the form

$\rho=\frac{M}{1+M}$

$\rho$ - strength of cell alignment (mean vector length of wrapped normal distribution describing the cell orientation distribution), $M$ - guidance cue magnitude

where the guidance cue magnitude is some function of descriptive variables relevant to the particular cue, such as strain anisotropy for the mechanical guidance cue, and ridge height and pitch for the structural guidance cue:

$M=F($ cue variables $)$

\section{Mechanical Guidance Cue}

For the mechanical cue data, we assumed the guidance cue magnitude is proportional to the strain anisotropy, which results in a function of the form 
$\rho_{m}=\frac{\Delta \varepsilon}{K_{m}+\Delta \varepsilon}$

$\rho_{m}$ - strength of cell alignment stimulated by mechanical guidance cue (cyclic uniaxial strain), $\Delta \varepsilon$ - strain anisotropy as defined in Eqn. 4.5, $K_{m}$ - saturation curve shape parameter

We modified this equation for implementation in the agent-based model. Previously in the agent-based model, we computed the mechanical guidance cue vector as

$\varepsilon_{n}=\vec{n}^{T}(\overline{\bar{\varepsilon}} \vec{n})$

$\vec{v}_{m, 2 \theta}=\frac{1}{2 \pi} \int_{-\pi}^{\pi} \varepsilon_{n}\left(R_{c e l l}, \theta\right) \vec{n}(2 \theta) d \theta$

$\bar{\theta}_{m}=\frac{1}{2} \angle \vec{v}_{m, 2 \theta}$

$M_{m}=\left\|\vec{v}_{m, 2 \theta}\right\|$

$\vec{v}_{m}=M_{m} \cos \left(\bar{\theta}_{m}\right) \vec{e}_{x}+M_{m} \sin \left(\bar{\theta}_{m}\right) \vec{e}_{y}$

$\varepsilon_{n}-$ strain normal to cell surface, $\vec{n}$ - unit outward normal vector from cell surface, $\overline{\bar{\varepsilon}}-$ strain tensor acting on cell, $\vec{v}_{m, 2 \theta}-2 \theta$-transformed mechanical guidance cue vector, $R_{\text {cell }}-$ cell radius, $\bar{\theta}_{m}-$ mechanical guidance cue orientation, $M_{m}-$ mechanical guidance cue magnitude as defined in the agent-based model, $\vec{v}_{m}$ - mechanical guidance cue vector

which defined a vector oriented along the axis of maximum strain with magnitude $\left(M_{m}\right)$ proportional to the strain anisotropy $(\Delta \varepsilon)$. Thus, we simply scaled $K_{m}$ in order to substitute $M_{m}$ in place of $\Delta \varepsilon$ in the equation for $\rho_{m}$ : 
$\rho_{m}=\frac{M_{m}}{K_{m}^{\prime}+M_{m}}$

For comparison, the previous equation for the strength of cell alignment stimulated by the mechanical cue used in the agent-based model simply normalized the mechanical cue magnitude by the maximum value possible in the model:

$\rho_{m}^{\text {old }}=\frac{M_{m}}{M_{m, \max }}$

$\rho_{m}^{\text {old }}$ - strength of cell alignment stimulated by mechanical guidance cue as defined previously in the agent-based model of infarct healing, $M_{m}$ - mechanical guidance cue magnitude as defined in the agent-based model, $M_{m, \max }$ - normalization factor for the mechanical guidance cue magnitude as defined in the agent-based model

\section{Structural Guidance Cue}

For the structural cue data, we had to handle two independent variables, ridge height and ridge pitch. Intuitively, we expect no cell alignment as the spacing of the ridges approaches zero or infinity, or as the height of the ridges approaches zero. In all of these limits, the surface appears flat. One way to capture these trends is to write $\rho_{s}$ in terms of height and pitch as

$\rho_{s}=\frac{1}{1+\frac{1}{H}\left(\frac{K_{S 1}}{P}+K_{S 2} P\right)}$

$\rho_{s}$ - strength of cell alignment stimulated by structural guidance cue, $H$ - ridge height, $P-$ ridge pitch, $K_{s 1}$ and $K_{s 2}$ - structural guidance cue saturation curve shape parameters 
We modified this equation for implementation in the agent-based model. Previously in the agent-based model, we computed the structural guidance cue vector as

$\vec{v}_{S, 2 \theta}=\frac{\int_{-\pi}^{\pi} N_{f}(\theta) \vec{u}(2 \theta) d \theta}{\int_{-\pi}^{\pi} N_{f}(\theta) d \theta}$

$\bar{\theta}_{S}=\frac{1}{2} \angle \vec{v}_{s, 2 \theta}$

$M_{s}=\left\|\vec{v}_{s, 2 \theta}\right\|$

$\vec{v}_{s}=M_{s} \cos \left(\bar{\theta}_{s}\right) \vec{e}_{x}+M_{s} \sin \left(\bar{\theta}_{s}\right) \vec{e}_{y}$

$\vec{v}_{s, 2 \theta}-2 \theta$-transformed structural guidance cue vector, $N_{f}$ - number of fibers located within the cell boundary, $\vec{u}$ - unit vector, $\bar{\theta}_{s}$ - structural guidance cue orientation, $M_{s}$ - mechanical guidance cue magnitude as defined in the agent-based model, $\vec{v}_{S}$ - structural guidance cue vector

which defined a vector oriented along the axis of matrix fiber alignment with magnitude $\left(M_{S}\right)$ equal to the strength of alignment of the matrix fibers. Because $M_{S}$ was already bounded between 0 and 1, the previous equation for the strength of cell alignment stimulated by the structural cue was simply defined as

$\rho_{s}^{\text {old }}=M_{S}$

$\rho_{s}^{\text {old }}$ - strength of cell alignment stimulated by structural guidance cue as previously defined in the agent-based model of infarct healing, $M_{S}$ - mechanical guidance cue magnitude as defined in the agent-based model, equal to the strength of alignment of matrix fibers 
This equation accounted for the effect of the strength of fiber alignment on the strength of cell alignment, but did not account for the effect of fiber size or fiber spacing. We used the microridge data to estimate these effects. We imagined that an array of parallel ridges is like an array of parallel collagen fibers. By this analogy, ridge height $(H)$ approximates collagen fiber diameter $\left(D_{c f}\right)$ and ridge pitch $(P)$ approximates collagen fiber spacing $\left(S_{c f}\right)$. These terms can be related to collagen area fraction, which is the relevant term in the agent-based model. For an array of parallel fibers, the area fraction is

$F_{c f}=\frac{D_{c f}}{S_{c f}} \sim \frac{H}{P}$

$F_{c f}$ - collagen fiber area fraction, $D_{c f}-$ collagen fiber diameter, $S_{c f}$ - collagen fiber spacing, $H$ - ridge height, $P$ - ridge pitch

If we assume that the fiber spacing in infarct scar during the first few weeks of healing does not become small enough to approach the flat surface limit, we can drop the $K_{s 1} / P$ term from Equation 4.16, then substitute $F_{c f}$ in place of $H / P$, and reintroduce the dependence on $M_{s}$ that was in $\rho_{s}^{\text {old }}$ to arrive at

$\rho_{s}=\frac{M_{s} F_{c f}}{K_{S}+F_{c f}}$

$\rho_{s}$ - strength of cell alignment stimulated by structural guidance cue, $M_{s}$ - mechanical guidance cue magnitude as defined in the agent-based model, equal to the strength of alignment of matrix fibers, $F_{c f}$ - collagen fiber area fraction, $K_{s}$ - structural guidance cue saturation curve shape parameter 
Simultaneous Guidance Cues

For the simultaneous cue data, the challenge was to determine how each individual guidance cue contributes to the resultant cell orientation distribution. We considered two possibilities: 1. the resultant cell orientation distribution is a weighted sum of the cell orientation distributions that would be observed for each individual guidance cue, defined as

$\varphi_{r}(\theta)=\omega_{s} \varphi_{s}(\theta)+\omega_{m} \varphi_{m}(\theta)$

$\omega_{s}+\omega_{m}=1$

$\varphi_{i}(\theta)=\frac{1}{2}\left[\varphi_{w n}\left(\theta: \rho_{i}, \bar{\theta}_{i}\right)+\varphi_{w n}\left(\theta: \rho_{i}, \bar{\theta}_{i}+\pi\right)\right]$ $\varphi_{r}-$ resultant cell orientation probability density function, $\varphi_{i}-$ cell orientation probability density function of guidance cue i, $\varphi_{w n}$ - wrapped normal probability density function, $\rho_{i}-$ strength of cell alignment (mean vector length) stimulated by guidance cue $i, \bar{\theta}_{i}-$ orientation of cell alignment (mean angle) stimulated by guidance cue i, $\omega_{i}$ - normalized weight factor of guidance cue $\mathrm{i}$ 
or 2. the resultant cell orientation distribution is prescribed by a resultant cell orientation vector that is a weighted sum of the cell orientation vectors for each individual guidance cue, defined as

$\varphi_{r}(\theta)=\frac{1}{2}\left[\varphi_{w n}\left(\theta: \rho_{r}, \bar{\theta}_{r}\right)+\varphi_{w n}\left(\theta: \rho_{r}, \bar{\theta}_{r}+\pi\right)\right]$

$\vec{c}_{r, 2 \theta}=\omega_{s} \vec{c}_{s, 2 \theta}+\omega_{m} \vec{c}_{m, 2 \theta}$

$\rho_{r}=\left\|\vec{c}_{r, 2 \theta}\right\|^{1 / 4}$

$\bar{\theta}_{r}=\frac{1}{2} \angle \vec{c}_{r, 2 \theta}$

$\omega_{s}+\omega_{m}=1$

$\vec{c}_{i, 2 \theta}=\rho_{i}{ }^{4} \cos \left(2 \bar{\theta}_{i}\right) \vec{e}_{x}+\rho_{i}{ }^{4} \sin \left(2 \bar{\theta}_{i}\right) \vec{e}_{y}$

$\varphi_{r}-$ resultant cell orientation probability density function, $\varphi_{w n}-$ wrapped normal probability density function, $\rho_{r}$ - strength of cell alignment (mean vector length) stimulated by resultant guidance cue, $\bar{\theta}_{r}$ - orientation of cell alignment (mean angle) stimulated by resultant guidance cue, $\quad \vec{c}_{r, 2 \theta}-2 \theta$-transformed cell orientation vector of resultant guidance cue, $\vec{c}_{i, 2 \theta}-2 \theta$ transformed cell orientation vector of guidance cue i, $\omega_{i}$ - normalized weight factor of guidance cue i, $\rho_{i}$ - strength of cell alignment (mean vector length) stimulated by guidance cue i, $\bar{\theta}_{i}-$ orientation of cell alignment (mean angle) stimulated by guidance cue i

where the angles are doubled so that anti-parallel guidance cues will add rather than subtract and the magnitudes are raised to the $4^{\text {th }}$ power before the vectors are combined, which are the proper transformations for bidirectional cues. The main difference between these approaches is that the distribution averaging method can predict a distribution with some cell orientations concentrated around the axis of the structural cue and some cell orientations concentrated around the axis of 
the mechanical cue, whereas the vector averaging method can only predict a distribution with cell orientations concentrated around a single alignment axis (Fig. 4.2).

We also needed a method of calculating the normalized weight factors $\left(\omega_{i}\right)$. Previously in the agent-based model, the normalized weight factors were defined as

$\omega_{i}=\frac{W_{i}}{\alpha+\sum W_{j} \rho_{j}}$

$W_{i}$ - weight factor of guidance cue i, $\alpha$ - tuning factor, $\rho_{i}$ - strength of cell alignment stimulated by guidance cue $\mathrm{i}$

We dropped $\alpha$ from this equation because its purpose-preventing the resultant strength of cell alignment from reaching a value of 1 -was accomplished through the use of saturation functions for the strength of cell alignment stimulated by individual guidance cues. We then considered the possibility that the terms in the numerator and denominator are nonlinear functions of the variables that describe the guidance cues, such as strain anisotropy and collagen area fraction:

$\omega_{i}=\frac{f(\text { variables describing cue } i)}{\sum f(\text { variables describing cue } j)}$ 


\section{Cell Migration}

We sought a method of predicting the migration behavior of fibroblasts in response to guidance cues. We assumed that any bias in the migration behavior of fibroblasts in response to guidance cues is due entirely to the bias in the shape of the cell orientation distribution. We simulated fibroblast migration as a series of displacements with constant magnitude $(d)$ and frequency $(f)$. The orientation of each displacement was determined by randomly sampling the cell orientation distribution prescribed by the guidance cues. For each cell mean vector length (strength of cell alignment) reported by Dickinson et al. (1994), we calculated the corresponding cell orientation distribution, simulated cell migration tracks, and calculated mean squared displacement curves. We then found random migration coefficients $\left(U, U_{x}, U_{y}\right)$ by fitting the mean squared displacement curves to the persistent random walk equations (Dickinson et al., 1994):

$\left\langle x^{2}+y^{2}\right\rangle=4 U\left[t-\tau\left(1-e^{-t / \tau}\right)\right]$

$\left\langle x^{2}\right\rangle=2 U_{x}\left[t-\tau_{x}\left(1-e^{-t / \tau_{x}}\right)\right]$

$\left\langle y^{2}\right\rangle=2 U_{y}\left[t-\tau_{y}\left(1-e^{-t / \tau_{y}}\right)\right]$

$U$ - random migration coefficient for 2D motion, $U_{i^{-}}$random migration coefficient for motion along the $\mathrm{i}$-axis, $\tau$-persistence time for $2 \mathrm{D}$ motion, $\tau_{i}$ - persistence time for motion along the i-axis, $t$-time, $x$-displacement along the $x$-axis (fiber axis), $y$-displacement along the $y$ axis (cross-fiber axis)

We iterated guesses of the displacement magnitude and frequency to solve for values that reproduced the experimental random migration coefficients for all cell mean vector lengths. 


\subsection{RESULTS}

\section{Calculation of Strength of Cell Alignment Stimulated by Guidance Cues}

We defined the strength of cell alignment as the mean vector length of the wrapped normal probability density function describing the cell orientation distribution. The literature studies we collected reported strength of cell alignment using various other measures. For each of these measurements, we solved for the corresponding mean vector length by inferring the underlying cell orientation distribution from which the measurement must have been derived. We were able to fit all of the literature data by assuming the underlying cell orientation distributions had the general shape of two identical wrapped normal distributions offset by 180 degrees and solving for the mean angle and mean vector length that specified the shape (Fig. 4.3). This approach also worked for the experimental data set with simultaneous structural and mechanical guidance cues acting along perpendicular axes (Fig. 4.3f).

\section{Mechanical Guidance Cue}

We obtained cell alignment data from 5 studies that applied $1 \mathrm{~Hz}$ cyclic uniaxial strain at varying strain amplitudes to cells plated on elastic membranes (Wang \& Grood, 2000; Neidlinger-Wilke et al., 2001; Loesberg et al., 2005; Kaunas \& Hsu, 2009; Prodanov et al., 2010). The axis of cell alignment (mean angle) was perpendicular to the axis of applied strain. The strength of cell 
alignment (mean vector length) increased asymptotically toward the maximum value of 1 as the strain anisotropy increased. We fit the function

$\rho_{m}=\frac{\Delta \varepsilon}{K_{m}+\Delta \varepsilon}$

to the data and determined the value of the parameter $K_{m}=0.0148$ (Fig. 4.4).

Rat cryoinfarcts located on the anterior wall of the left ventricle experienced average circumferential and longitudinal strains of 0.065 and -0.013 acutely after infarction (Fomovsky et al., 2012) and therefore had a strain anisotropy of $\Delta \varepsilon=0.078$ and expected strength of cell alignment due only to the mechanical guidance cue of $\rho_{m}=0.84$. Previously in the agent-based model of infarct healing we used a value of $\rho_{m}^{\text {old }}=1$.

\section{Structural Guidance Cue}

We obtained cell alignment data from 8 studies that plated cells on substrates with micro-ridges of varying height and pitch (Clark et al., 1990; Sutherland et al., 2005; Loesberg et al., 2005, 2007; Kim et al., 2009; Pot et al., 2010; Sun et al., 2010; Prodanov et al., 2010). The axis of cell alignment (mean angle) was parallel to the axis of ridge alignment. The strength of cell alignment (mean vector length) approached zero as height approached zero, as pitch approached zero, and as pitch approached infinity. At constant height, the strength of cell alignment plateaued at a pitch on the order of 1 to $10 \mu \mathrm{m}$. At constant pitch, the strength of cell alignment asymptotically approached its maximum value with increasing height. We first visualized these trends by fitting a regularized (smoothed) interpolation surface to the data (Fig. 4.5a). We then fit the function 
$\rho_{S}=\frac{1}{1+\frac{1}{H}\left(\frac{K_{S 1}}{P}+K_{S 2} P\right)}$

to the data and determined the values of the parameters $K_{s 1}=0.00203 \mathrm{~nm}^{2}$ and $K_{s 2}=4240$ (Fig. 4.5b). Finally, we truncated the data set by retaining data with pitch values greater than $400 \mathrm{~nm}$ in order to avoid the low pitch decay of the strength of cell alignment, fit the simplified function

$\rho_{S}=\frac{\left(\frac{H}{P}\right)}{K_{S}+\left(\frac{H}{P}\right)}$

to the data, and determined the value of the parameter $K_{s}=0.0088$ (Fig. $4.5 \mathrm{e}$ ).

Normal rat myocardium has a collagen area fraction of $F_{c f}=0.03$. After infarction, collagen area fraction may decline by approximately $50 \%$ before rising. Using

$\rho_{s}=\frac{M_{s} F_{c f}}{K_{S}+F_{c f}}$

the minimum expected strength of cell alignment due only to the structural guidance cue was $\rho_{s, \min }=0.63 M_{s}$. Using collagen area fraction measurements from rat ligation infarcts, by 1 week after infarction $F_{c f}=0.09$ and $\rho_{s}=0.91 M_{s}$, and by 3 weeks after infarction $F_{c f}=0.21$ and $\rho_{s}=0.96 M_{s}$. Previously in the agent-based model of infarct healing we used $\rho_{s}^{\text {old }}=M_{s}$.

\section{Simultaneous Guidance Cues}

We obtained cell alignment data from the study by Prodanov et al. (2010) that applied cyclic uniaxial strain to cells plated on micro-ridged substrates. Strain was applied along the ridges, which generated a mechanical guidance cue perpendicular to the structural guidance cue. In the absence of cyclic strain, cells were aligned along the structural cue axis. At the maximum strain 
anisotropy of 0.12 , cells were aligned along the mechanical cue axis. As the magnitude of strain anisotropy increased from 0 to 0.12 , the strength of cell alignment (mean vector length) decreased and then increased, and the axis of cell alignment (mean angle) transitioned from the structural cue axis to the mechanical cue axis (Fig. 4.6c).

Prodanov et al. (2010) also measured the strength of cell alignment as a function of cyclic uniaxial strain applied to smooth substrates. For each magnitude of strain anisotropy acting on the micro-ridged substrates, we knew the expected strength of cell alignment due to the mechanical guidance cue alone and the expected strength of cell alignment due to the structural guidance cue alone (Fig. 4.6a). We tested if the cell orientation distributions observed when the guidance cues acted simultaneously could be computed from the individual guidance cues using the distribution averaging method or the vector averaging method. The vector averaging method $\left(\operatorname{RMSE}=1.5^{\circ}\right)$ and the distribution averaging method $\left(\mathrm{RMSE}=2.4^{\circ}\right)$ were able to reproduce the experimental data (Fig. 4.6b).

Using the vector averaging method, we obtained normalized weight factors $\left(\omega_{i}\right)$ for the mechanical and structural guidance cues. The normalized weight factor for the mechanical guidance cue increased as a function of strain anisotropy. We fit the function

$\omega_{m}=\frac{\left(W_{m} \Delta \varepsilon\right)^{c}}{\left(W_{s} F_{c f}\right)^{c}+\left(W_{m} \Delta \varepsilon\right)^{c}}$

to the data (using $F_{c f} \sim H / P=0.25$ ) and determined the values of the parameters $W_{m} / W_{s}=$ 5.85 and $c=2.20$ (Fig. 4.6d). 
Immediately after infarction the expected collagen area fraction is $F_{c f}=0.03$, and three weeks after infarction the expected collagen area fraction is $F_{c f}=0.21$. For a rat cryoinfarct located on the anterior wall of the left ventricle, the expected strain anisotropy is $\Delta \varepsilon=0.078$. Throughout the bulk of the infarct (neglecting the possible chemical guidance cue due to chemokine gradients at the edge of the infarct), the expected ratio of normalized weight factors is $\omega_{m} / \omega_{s}=$ 400 immediately after infarction and 5.5 three weeks after infarction. Previously in the agentbased model the ratio was a constant equal to $\omega_{m}^{\text {old }} / \omega_{s}^{\text {old }}=W_{m}^{\text {old }} / W_{s}^{\text {old }}=3.5$.

\section{Cell Migration}

We obtained cell alignment data and cell migration data from the study by Dickinson et al. (1994) that seeded fibroblasts into collagen gels with varying strengths of collagen fiber alignment. We tested if the migration behavior could be modeled as a biased random walk, that is, if fibroblast migration could be simulated as a series of displacements of magnitude $d$ and frequency $f$, where the orientation of each displacement is determined by randomly sampling a non-uniform orientation distribution. Using a displacement magnitude of $d=17 \mu m$ and frequency $f=1 h r^{-1}$, and setting the displacement orientation distribution equal to the cell orientation distribution for a given strength of cell alignment $\left(\rho_{s}\right)$, the simulated migration tracks predicted random migration coefficients in good agreement with the experimental data. As the strength of cell alignment increased, the random migration coefficient quantifying migration along the fiber axis increased, and the random migration coefficient quantifying migration along the cross-fiber axis decreased (Fig. 4.7). 
In general, any pair of $d$ and $f$ (or $S=d f$ and $\tau=(2 f)^{-1}$ ) that satisfied

$U=\frac{1}{2}\left(U_{x}+U_{y}\right)=\frac{1}{2} S^{2} \tau=\frac{1}{4} d^{2} f=1.2 \mu \mathrm{m}^{2} / \mathrm{min}$

$U$ - random migration coefficient, $S$ - migration speed, $\tau$-persistence time, $d$-displacement magnitude, $f$-displacement frequency

would have predicted the same $U_{x}$ and $U_{y}$ curves. The specific choice of $d$ and $f$ must be derived from more detailed information in the migration track data, such as the shapes of the mean squared displacement curves. The paper by Dickinson et al. contained only a pair of example mean squared displacement curves, which we used to roughly estimate $f$.

\section{Revised Agent-based Model of Infarct Healing}

We updated the agent-based model of infarct healing to include the equations that we derived from the literature data, as discussed above. We then simulated healing of circular infarcts subjected to either uniaxial circumferential strain (anterior wall cryoinfarct) or equibiaxial strain (apical cryoinfarct). For the uniaxial strain case, we simulated healing of the infarct at different transmural depths by prescribing an initial fiber orientation of 0,30 , or 60 degrees. We allowed collagen fiber remodeling via either aligned deposition only or rotation only. The cell mean vector length, collagen mean vector length, and collagen mean angle predictions of the revised model were similar to the previous model (Fig. 4.8-9). 


\subsection{DISCUSSION}

\section{Mechanical Guidance Cue}

The alignment of cells in response to cyclic strain was consistent across several cell types. The axis of cell alignment was perpendicular to the axis of applied strain, and the strength of cell alignment approached its maximum asymptotically as the strain anisotropy increased. We formulated equations for predicting the cell orientation distribution expected for a given strain field. Using the systolic strains experienced by cryoinfarcts located on the anterior wall of the left ventricle of rats, the model predicted cell alignment strong enough to explain the collagen alignment observed in the cryoinfarct scars (Fomovsky et al., 2012). This supports our conclusion from Chapter 2 that mechanical guidance cues can be important during infarct healing.

Models predicting the alignment of cells in response to cyclic strain have been published previously (Kaunas \& Hsu, 2009; Kang et al., 2011). These tend to be mechanistic and require detailed computation. In contrast, our approach is phenomenologic and very simple. This makes it attractive for use in cell-based models that predict tissue-scale events, where subcellular events may be too cumbersome to compute. One important application would be to predict the evolution of tissue properties in situations where mechanical cues can vary over time. For example, during infarct healing, as cells remodel the infarct microstructure, the strains acting on the infarct may change, which will then change cell alignment and alter the course of structural remodeling. We address this topic further in Chapter 6. Another application would be to predict 
the consequences of an intervention that changes the response of cells to strain. For example, Kaunas et. al. (2005) showed that expression of V14-Rho in bovine aortic endothelial cells reduced the strength of cell alignment in response to cyclic strain. Such an intervention could be modeled by changing the parameter $K_{m}$.

\section{Limitations}

We did not investigate how the strength of cell alignment depends on the frequency of cyclic strain. During infarct healing, we expect the effects of transient heart rate fluctuations to average out on our time scale of interest (days to weeks).

All of the literature studies applied stretch along a single axis. A wider range of stretch protocols should be investigated in order to determine if all data truly collapse onto a single curve of mean vector length versus strain anisotropy.

We have assumed that the strength of cell alignment in response to cyclic uniaxial strain is the same in $3 \mathrm{D}$ as it is in $2 \mathrm{D}$. Yet, cells can exhibit behavior in $3 \mathrm{D}$ that is different from their behavior in 2D. For example, cells align parallel to the axis of applied strain in 3D (Henshaw et al., 2006; Lee et al., 2008; Pang et al., 2011), whereas cells align perpendicular to the axis of applied strain in 2D (Neidlinger-Wilke et al., 2001). In practice, it is difficult to investigate the alignment response in 3D because cell culture scaffolds are typically made of fibrous materials in which mechanical and structural cues are usually coupled. Thus, we relied on data gathered from studies that used 2D substrates. 


\section{Structural Guidance Cue}

The alignment of cells on micro-ridged substrates was consistent across several cell types. The axis of cell alignment was parallel to the ridges and the strength of cell alignment depended predictably on the groove dimensions - the strength of cell alignment approached zero as the ridge height approached zero, as the ridge pitch approached zero, and as the ridge pitch approached infinity. We formulated equations for predicting the strength of cell alignment and the cell orientation distribution expected for a given substrate topography. We drew an analogy between micro-ridges and extracellular matrix fibers in order use the model to predict cell alignment in collagenous tissues as a function of collagen area fraction. Using collagen area fraction measurements from ligation infarcts in rats, the model predicted the strength of cell alignment would be insensitive to collagen area fraction by 1 week after infarction. This supports our original approach that did not account for any effect of collagen area fraction on the strength of cell alignment during infarct healing.

Although the data suggested effects of fiber size and fiber density on the strength of cell alignment are negligible during infarct healing, they may be important in other tissues where the fiber area fraction is low, that is, the fibers are small, sparse, or small and sparse. In this regime, the strength of cell alignment should be lower than what would be expected without accounting for fiber size and density. These effects could also be relevant for interpreting the results of molecular and genetic studies that alter the assembly and packaging of collagen fibers. For example, decorin can regulate the size and density of collagen fibers (Weis et al., 2005). Our 
model could potentially predict some effects of altered proteoglycan concentrations on cell and tissue structure.

\section{Limitations}

We significantly reduced the complexity of the micro-ridge data set in order to obtain equations that we could use with our agent-based model. The strength of cell alignment should depend on three variables: ridge height, ridge width, and ridge pitch (spacing). Although we did not obtain sufficiently diverse data to investigate the effect of ridge width, we found height and pitch to be adequate for predicting the strength of cell alignment. We reduced the data further to a single independent variable, the ratio of height to pitch. This ratio could predict the average trend of the data, but did not completely collapse it onto a single curve. Finally, we related the ratio of height over pitch to collagen area fraction. It is possible that the value of $K_{s}$ we obtained for ridges is different from the value for collagen fibers. Higher values of $\mathrm{K}_{\mathrm{s}}$ would lower the expected strength of cell alignment due to structural guidance cues during infarct healing, but this would have little impact given the dominance of the mechanical guidance cue.

The micro-ridge data set only provides information about how strongly cells will align with perfectly aligned ridges, but in tissues, cells may be in contact with a disorganized array of fibers. We assumed that the size and spacing of fibers determines how strongly the cell orientation distribution will match the ridge or fiber orientation distribution. Thus, we included the fiber mean vector length $M_{S}$ in the equation for $\rho_{s}$ in order to scale the strength of cell 
alignment by the strength of fiber alignment. It will be important to determine the relationship between fiber and cell mean vector length experimentally.

\section{Simultaneous Guidance Cues}

\section{Method of Combining Guidance Cues}

We considered two methods of computing cell alignment in response to simultaneous guidance cues: distribution averaging and vector averaging. Prodanov et al. (2010) measured cell alignment in response to simultaneous mechanical and structural guidance cues. We were able to reproduce the cell alignment data from Prodanov et al. using the distribution averaging method or the vector averaging method. The finding that the vector-averaging method fit the experimental data just as well, if not better than the distribution averaging method, implies that the experimental orientation distributions did not strongly feature cell orientations concentrated around the axes of both of the guidance cues, which would have been the advantage of the distribution averaging method. However, this cannot be concluded definitively from the reported box and whisker plots.

Another study suggests that cell orientations are concentrated around a single alignment axis when guidance cues act simultaneously, which supports the vector averaging method (Ahmed et al., 2010). Yet another study suggests that cell orientations are concentrated around the axes of both of the individual guidance cues, which supports the distribution averaging method, but the data are few (Rajnicek et al., 2007). Additional data is needed in order to come to a definite 
conclusion, and this data must be in the form of a complete orientation histogram. An experiment similar to that by Prodanov et al. would provide much stronger evidence if orientation histograms were reported instead of box and whisker plots.

\section{Method of Computing Weight Factors}

The problem of computing normalized weight factors $\left(\omega_{i}\right)$ when guidance cues act simultaneously is an exciting direction of future work. Prodanov et al. (2010) and others (Rajnicek et al., 2007; Ahmed et al., 2010; Li et al., 2012) have published studies that provide a glimpse into the effect of simultaneous guidance cues on cell alignment, however, these studies have not explored nearly enough parameter space to provide a comprehensive picture of how guidance cues interact. Fortunately, Prodanov et al. studied mechanical and structural guidance cue magnitudes in ranges relevant to our infarct healing study. The data support our conclusion from Chapter 2 that mechanical cues are dominant during infarct healing.

\section{Limitations}

Because Prodanov et al. (2010) only varied the mechanical guidance cue magnitude (strain anisotropy) in their study, we were unable to determine how the equation for computing normalized weight factors should depend on variables describing the structural guidance cue. We guessed that there should be a term for collagen area fraction $\left(W_{s} F_{c f}\right)^{c}$ similar to the term we formulated for strain anisotropy $\left(W_{m} \Delta \varepsilon\right)^{c}$. Assuming our analogy between ridges and fibers holds (at least approximately), then the micro-ridged structure investigated corresponded to a 
fiber structure with an area fraction of $F_{c f} \sim H / P=0.25$. Consequently, we can expect the equation for computing normalized weight factors to work reasonably for similar structures, that is, for $F_{c f}$ or $H / P$ near 0.25 , but we have little confidence in the applicability of the equation to different structures. In the context of infarct healing, this means the equation for computing normalized weight factors may work reasonably beyond roughly 2 weeks after infarction $\left(F_{c f}=0.16\right)$, but not earlier when the collagen area fraction differs greatly from 0.25 .

Several modeling papers have proposed computational models for calculating the alignment of cells in response to simultaneous guidance cues (Wagle \& Tranquillo, 2000; McDougall et al., 2006), but no prior study has attempted an approach founded on empirical evidence as we have done. Yet, we still know very little. What are the independent variables that determine the relative weighting of guidance cues: strain anisotropy or strain amplitude; fiber size, spacing, or strength of fiber alignment? Do the weight factors depend on the angle between the guidance cues? Is it possible to have a guidance cue that stimulates a low strength of cell alignment but has high weight? For example, equibiaxial strain does not cause cell alignment, but is that because it has no effect or because it is disruptive of cell alignment? Can equibiaxial strain negate the alignment induced by aligned fibers? Can large, dense, randomly oriented fibers negate the alignment induced by uniaxial strain? These uncertainties in our approach to computing cell alignment in response to simultaneous guidance cues can be used to guide further experimentation. 


\subsection{CONCLUSION}

We used published experimental data to formulate and approach to predicting cell alignment in response to mechanical (uniaxial cyclic stretch), structural (aligned micro-ridges or fibers), or simultaneous mechanical and structural guidance cues. The literature data supported our approach to representing guidance cues as vectors, combining guidance cues as a weighted vector sum, and relating the resultant vector to a cell orientation probability distribution. The experimental data also supported the predictions of our agent-based model of infarct healing. Specifically, the data suggested that the strains acting on cryoinfarcts located on the anterior wall of the left ventricle should stimulate a high strength of cell alignment that has a high weight relative to the strength of cell alignment stimulated by structural guidance cues, and that we need not account for an effect of collagen area fraction on the strength of cell alignment stimulated by the structural guidance cue during infarct healing. We identified gaps in our knowledge about guidance cues and how they interact, and we propose continuing work using stretchable, microtextured substrates to cover yet unexplored parameter space. We emphasize the importance of reporting full cell orientation distributions when studying cell alignment. 


\subsection{FIGURES AND TABLES}

A

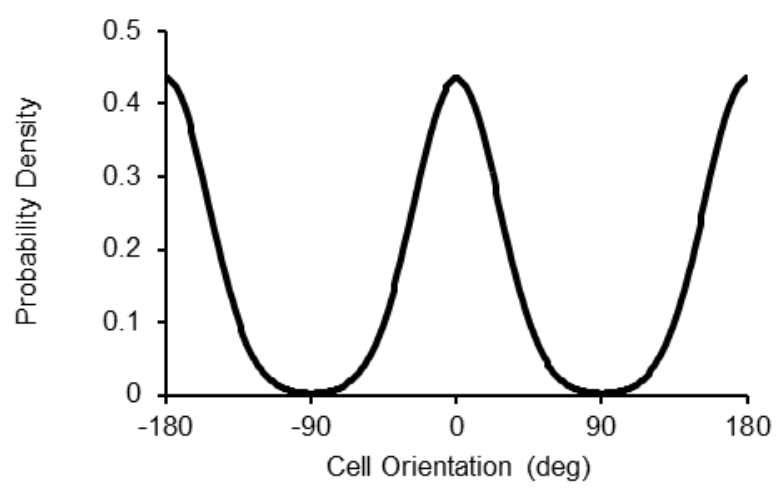

B

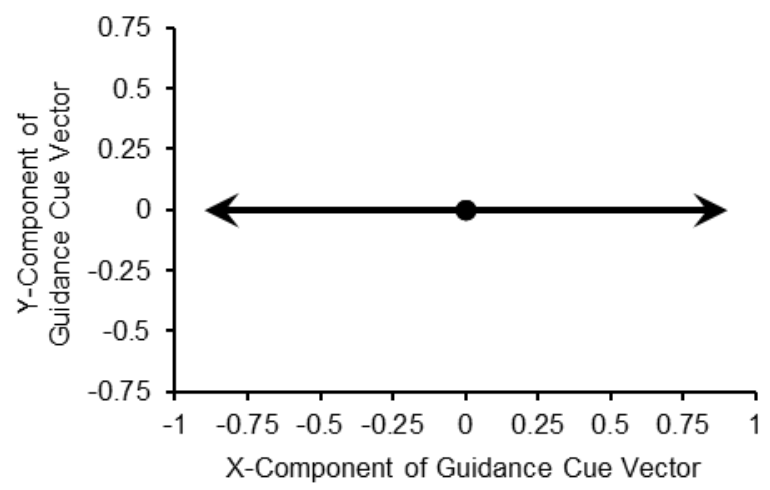

Figure 4.1. Example cell orientation probability distribution and vector representation. Bidirectional guidance cues, such as uniaxial strain and aligned fibers, cause cells to orient along a particular axis without preference for either of the two antiparallel directions along that axis. A bimodal distribution is expected, with cell orientations concentrated around each of the two antiparallel directions. A. This distribution can be modeled as an average of two wrapped normal probability density functions with mean angles offset by 180 degrees. The wrapped normal probability density function is parameterized by a mean angle, which prescribes the center of the peak of the distribution, and a mean vector length, which prescribes how strongly the distribution is concentrated about the mean angle. (The mean vector length is related to the circular standard deviation; a mean vector length of 1 corresponds to a circular standard deviation of 0 and a mean vector length of 0 corresponds to a circular standard deviation of $\infty$.) B. Together, the mean angle and mean vector length define a vector. This allows bidirectional guidance cues to be represented in a compact form as two antiparallel vectors of equal magnitude. 
A. Distribution Averaging

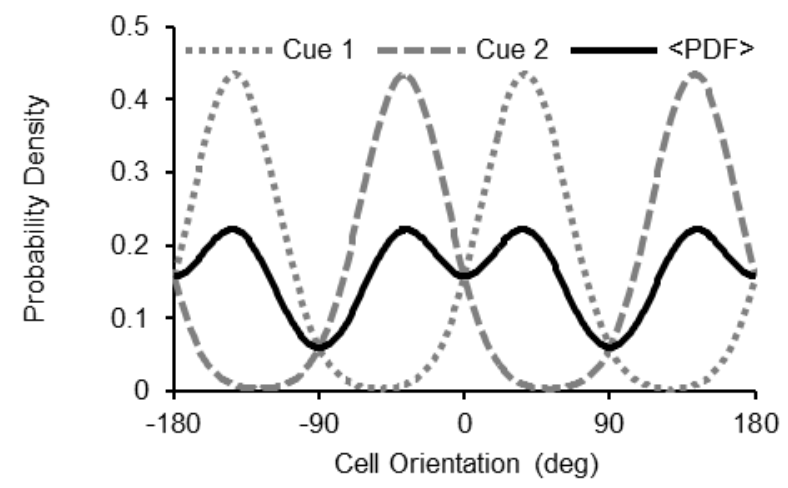

C. Vector Averaging

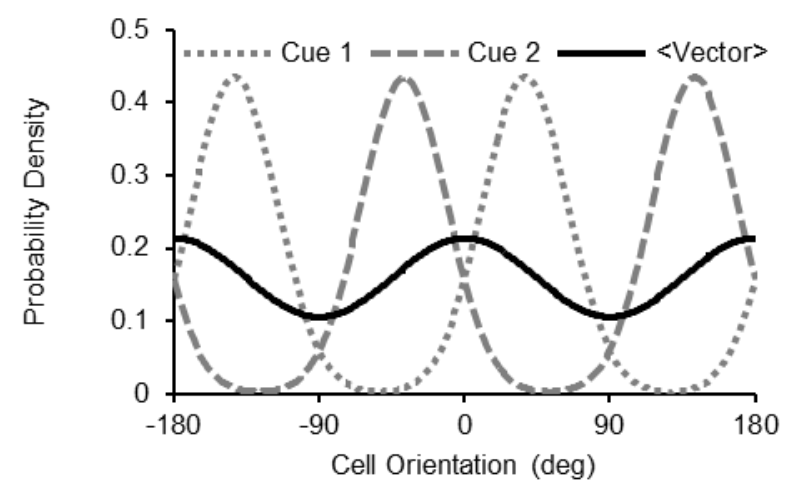

B. Vector Representation

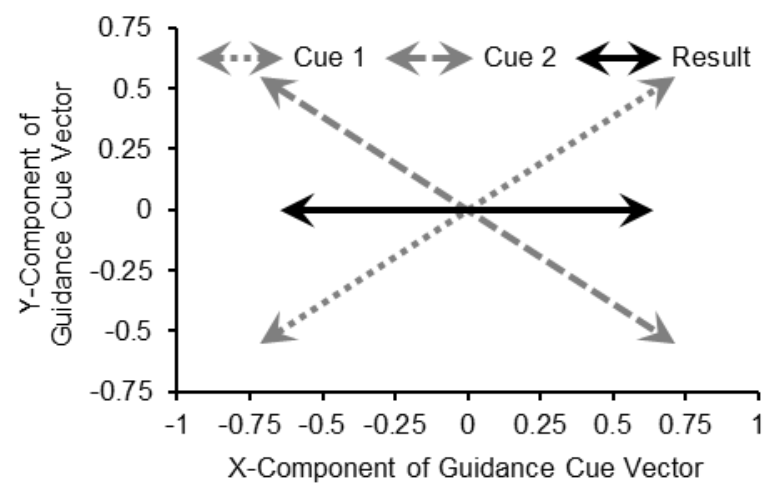

Figure 4.2. Example resultant cell orientation probability distributions. We considered two possible methods of computing the expected cell orientation distribution when two guidance cues act simultaneously. We hypothesized that if the cell orientation distributions for each individual guidance cue (Cue 1 and Cue 2) are known, then the resultant distribution can be determined either A. by computing the weighted sum of the individual distributions (distribution averaging method), or B. by computing the weighted sum of the representative mean vectors for each cue and then C. computing the orientation distribution associated with the resultant vector (vector averaging method). A. The distribution averaging method ( $\langle\mathrm{PDF}\rangle$ ) predicts concentrations of cell orientations around the axes of both of the individual guidance cues (Cue 1 and Cue 2). $\mathbf{C}$. The vector averaging method (<Vector $\rangle)$ predicts concentration of cell orientations around a single axis that lies between the axes of the individual guidance cues. 

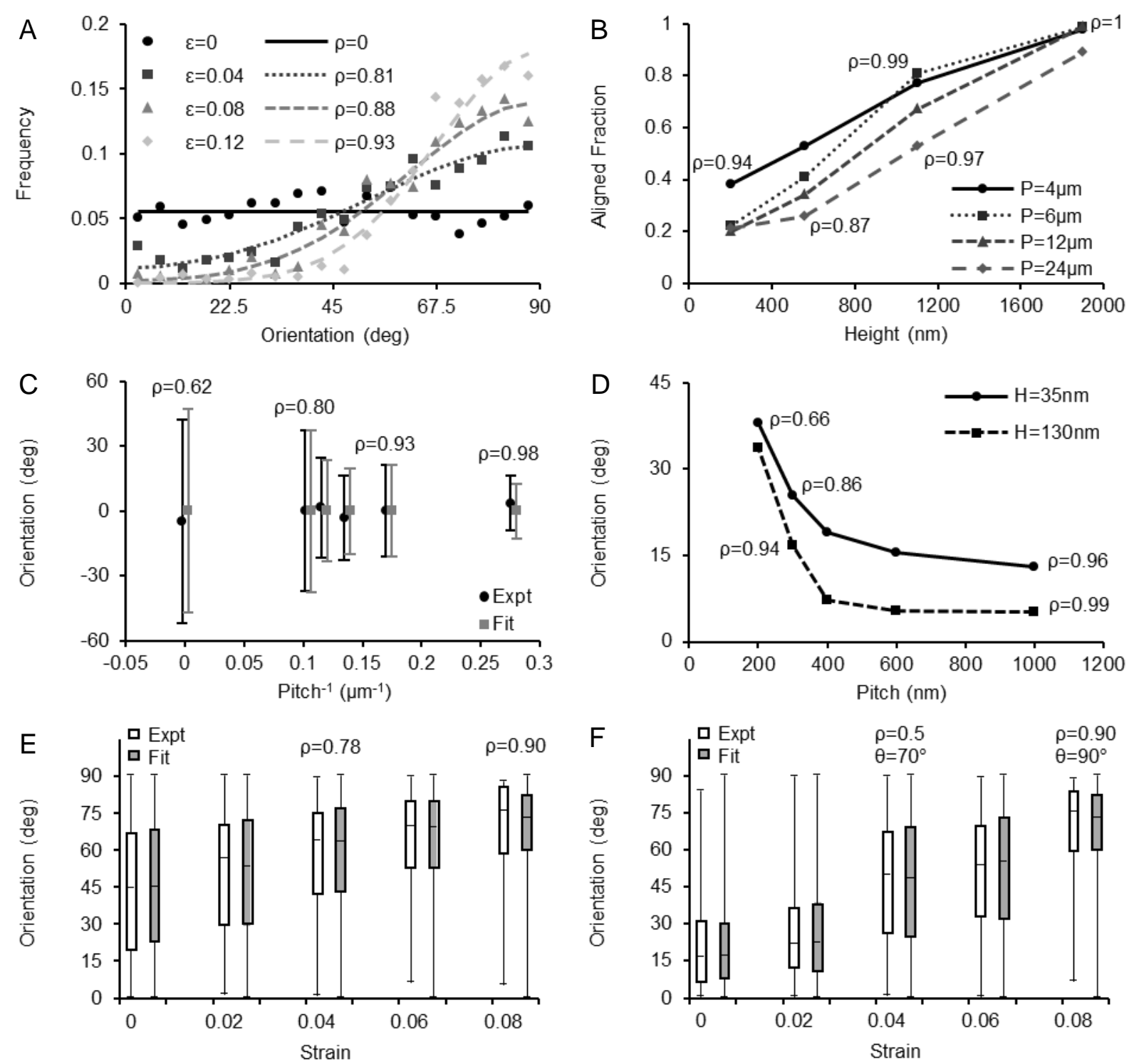

Figure 4.3. Calculation of strength of cell alignment. We found 5 studies that quantified the alignment of cells subjected to cyclic strain (mechanical guidance cue) and 8 studies that quantified the alignment of cells plated on micro-ridged surfaces (structural guidance cue). The literature studies reported a variety of metrics quantifying the strength of cell alignment. We converted all measurements to a mean vector length $(\rho)$ by inferring an underlying cell orientation distribution consistent with the measurement. A. Neidlinger-Wilke et al. reported cell orientation distributions for cyclic uniaxial strain amplitudes of 0 , $0.04,0.08$, and 0.12 . B. Clark et al. reported the percentage of cells oriented within 10 degrees of the axis of cell alignment for ridged substrates with $4,6,12$, or $24 \mu \mathrm{m}$ pitch and varying height. C. Kim et $a l$. reported the arithmetic mean and standard deviation of angles ranging from -90 to +90 degrees for ridged substrates with $400 \mathrm{~nm}$ height and varying pitch. D. Loesberg et al. reported the arithmetic mean of angles ranging from 0 to 90 degrees for ridged substrates with 35 or $130 \mathrm{~nm}$ height and varying pitch. E. Prodanov et al. reported orientation distribution quartiles for cyclic uniaxial strain amplitudes ranging from 0 to 0.08 . F. Prodanov et al. reported orientation distribution quartiles for cyclic uniaxial strain amplitudes ranging from 0 to 0.08 applied to ridged substrates with $600 \mathrm{~nm}$ pitch and $150 \mathrm{~nm}$ height. Strain was applied parallel to the ridges such that the structural guidance cue $\left(0^{\circ}\right)$ and mechanical guidance cue $\left(90^{\circ}\right)$ acted along perpendicular axes. 


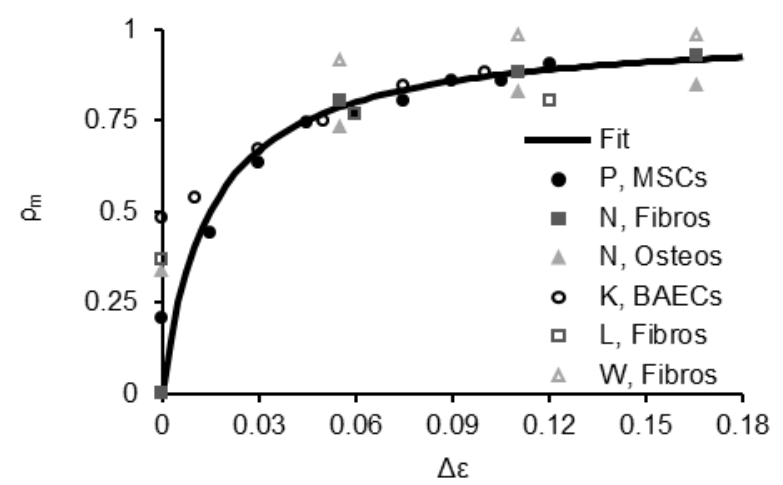

Figure 4.4. Mechanical guidance cue. We obtained cell alignment data from 5 studiesProdanov et al. (P), Neidlinger-Wilke et al. (N), Kaunas et al. (K), Loesberg et al. (L), and Wang et al. (W) - that applied $1 \mathrm{~Hz}$ cyclic uniaxial strain at varying strain amplitudes to cellsmesenchymal stem cells (MSCs), fibroblasts (Fibros), osteoblasts (Osteos), bovine aortic endothelial cells (BAECs) — plated on elastic membranes. The strength of cell alignment $\left(\rho_{m}\right)$ increased asymptotically toward the maximum value of 1 as the difference between principal strains $(\Delta \varepsilon)$ increased. The proposed function (Eqn. 4.38) captured this trend. 
A

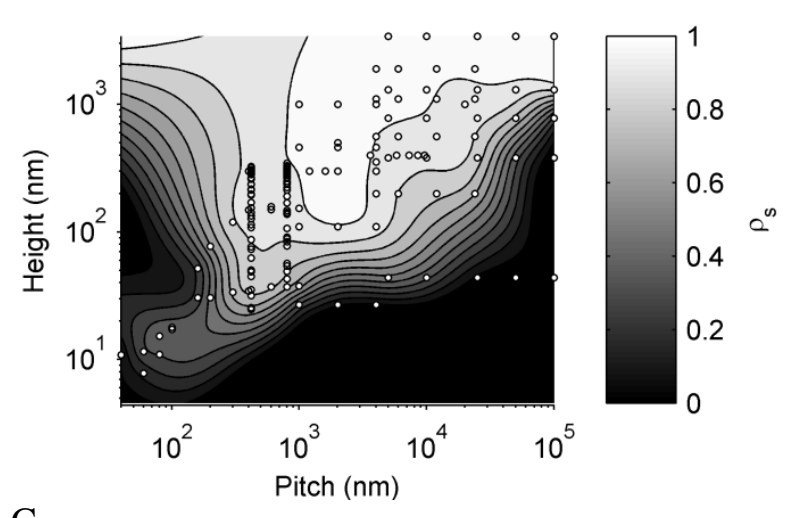

$\mathrm{C}$

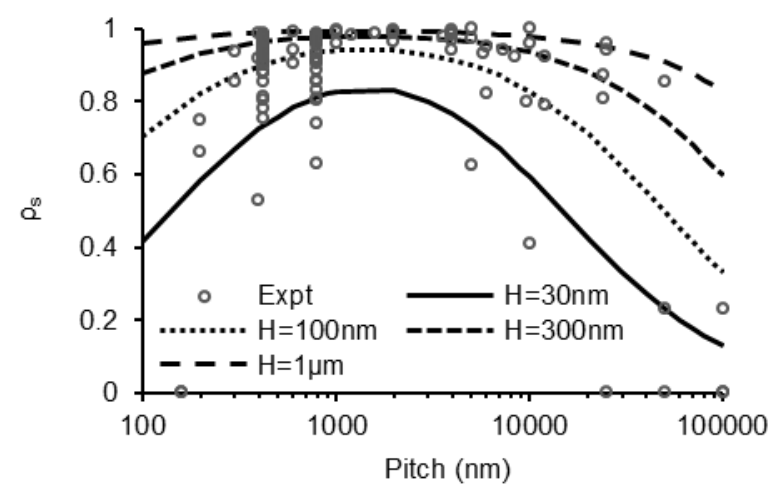

B

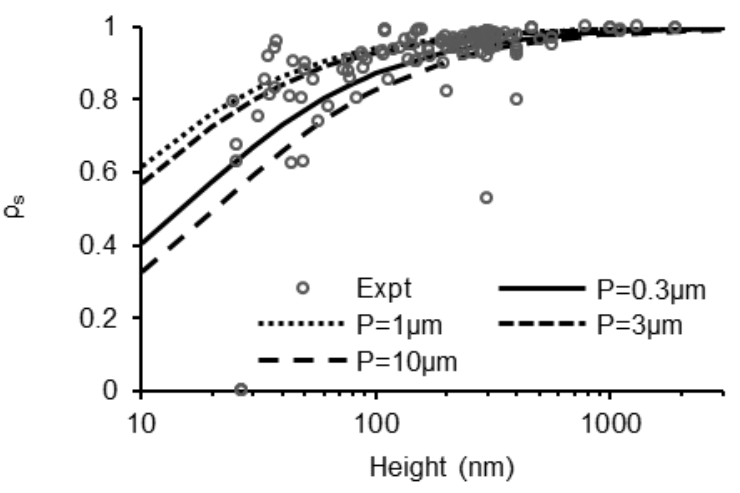

$\mathrm{D}$

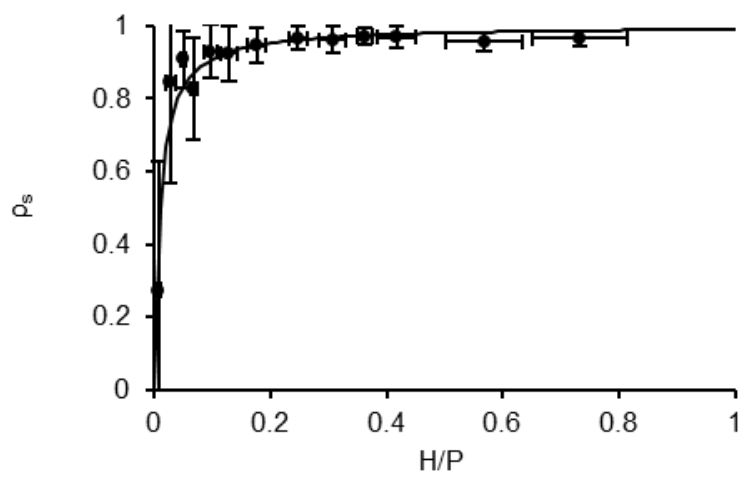

Figure 4.5. Structural guidance cue. We obtained cell alignment data from 8 studies (Prodanov et al., Kim et al., Loesberg et al., Loesberg et al., Sun et al., Sutherland et al., Pot et al., and Clark et al.) that plated cells on substrates with micro-ridges of varying height and pitch. A. We fit a regularized (smoothed) interpolation to the scattered literature data (white filled circles) in order to visualize the strength of cell alignment as a function of ridge dimensions. The expected trends toward zero alignment at low height, low pitch, and high pitch were apparent in the experimental data, as well as the plateau of the strength of cell alignment with increasing height. B and C. The proposed function (Eqn. 4.39) captured the general features of the scattered data. Plot $\mathbf{B}$ shows experimental data for pitches ranging from $0.3 \mu \mathrm{m}$ to $10 \mu \mathrm{m}$ along with function iso-curves at several pitch values in that range. Plot $\mathbf{C}$ shows experimental data for heights ranging from $30 \mathrm{~nm}$ to $1 \mu \mathrm{m}$ along with function iso-curves at several height values in that range. D. We truncated the data set below a pitch of $400 \mathrm{~nm}$ in order to avoid the low pitch decay of the guidance cue strength. We plotted the remaining data as a function of a single parameter, the ratio of ridge height to ridge pitch. The strength of cell alignment $\left(\rho_{s}\right)$ increased asymptotically toward the maximum value of 1 as the ratio $(H / P)$ increased. The proposed simplified function (Eqn. 4.40) captured this trend. 
A

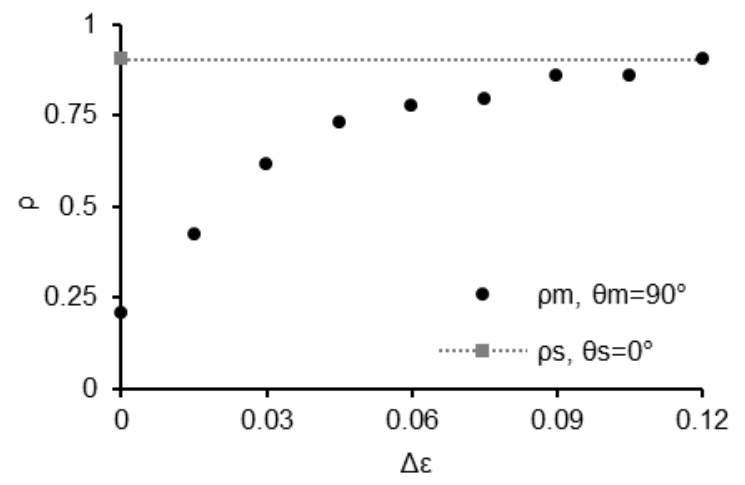

$\mathrm{C}$

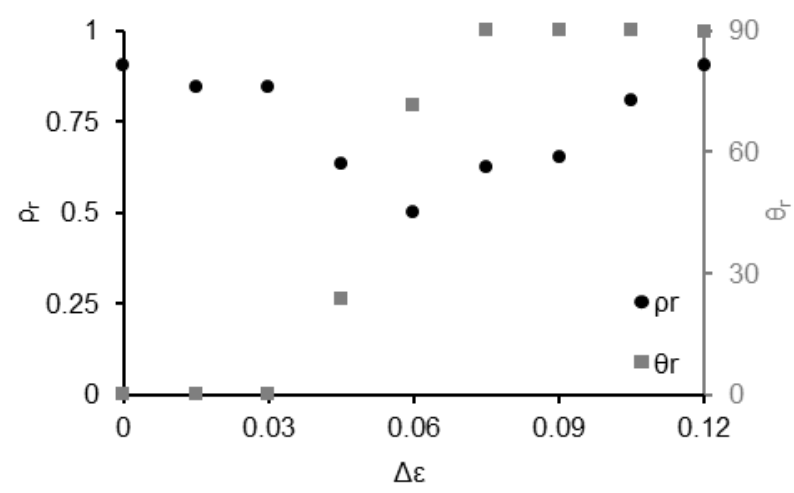

B

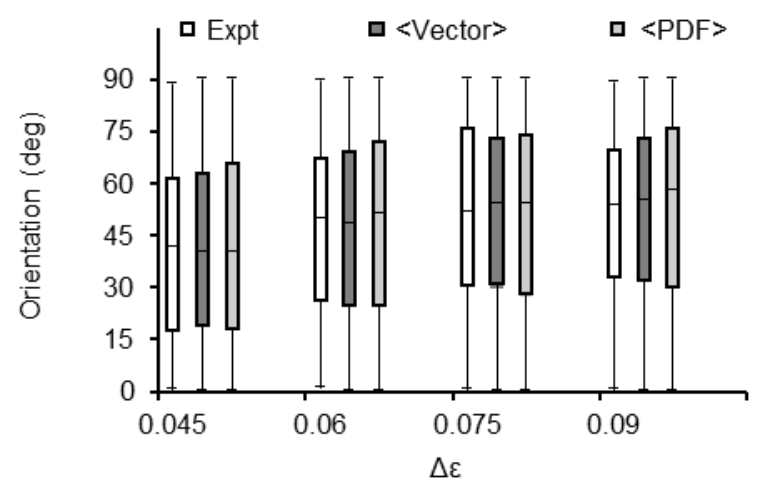

$\mathrm{D}$

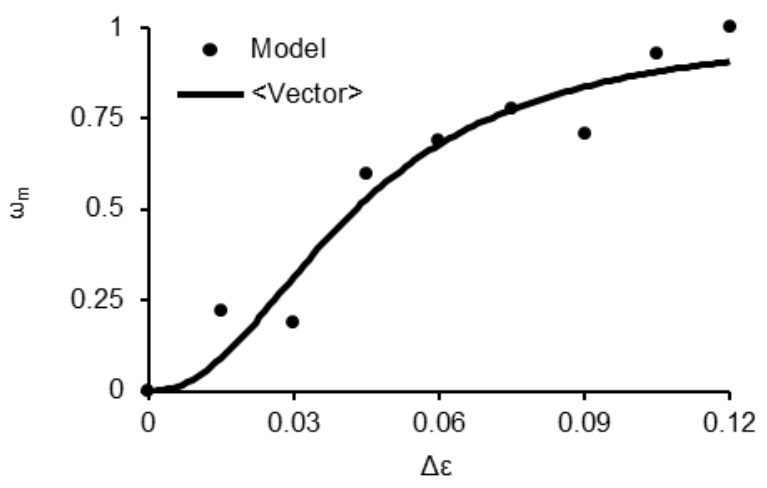

Figure 4.6. Simultaneous guidance cues. Prodanov et al. measured cell alignment for cyclic uniaxial strain amplitudes ranging from 0 to 0.08 applied to ridged substrates with $600 \mathrm{~nm}$ pitch and $150 \mathrm{~nm}$ height. Strain was applied parallel to the ridges such that the structural guidance cue (0 degrees) and mechanical guidance cue (90 degrees) acted along perpendicular axes. We tested if the cell orientation distributions observed when the guidance cues acted simultaneously could be computed from the individual guidance cues. A. From control data reported in the study, we determined the strength and orientation of cell alignment due only to the micro-ridges $\left(\rho_{s}\right.$ and $\left.\bar{\theta}_{s}\right)$ and the strength and orientation of cell alignment due only to cyclic uniaxial strain $\left(\rho_{m}\right.$ and $\left.\bar{\theta}_{m}\right)$. B. We then computed the resultant orientation distributions expected when the structural and mechanical guidance cues act simultaneously using either the vector averaging method $(\langle$ Vector $\rangle)$ or distribution averaging method $(\langle\mathrm{PDF}\rangle)$, and compared distribution quartiles to the experimental data. The vector averaging method $\left(\operatorname{RMSE}=1.5^{\circ}\right.$ ) and the distribution averaging method $\left(\mathrm{RMSE}=2.4^{\circ}\right.$ ) produced good matches to the experimental quartiles. The plot shows quartiles for the regime where neither guidance cue is dominant. $\mathbf{C}$ and $\mathbf{D}$. Using the vector averaging method, we found the normalized weight factors $\left(\omega_{i}\right)$ that related the cell orientation vectors of the individual cues (specified by $\rho_{i}$ and $\bar{\theta}_{i}$ ) to the resultant cell orientation vectors (specified by $\rho_{r}$ and $\bar{\theta}_{r}$ ). The normalized weight factor of the mechanical cue $\left(\omega_{m}\right)$ increased as a function of strain anisotropy. The proposed function (Eqn. 4.42) captured this trend. 
A

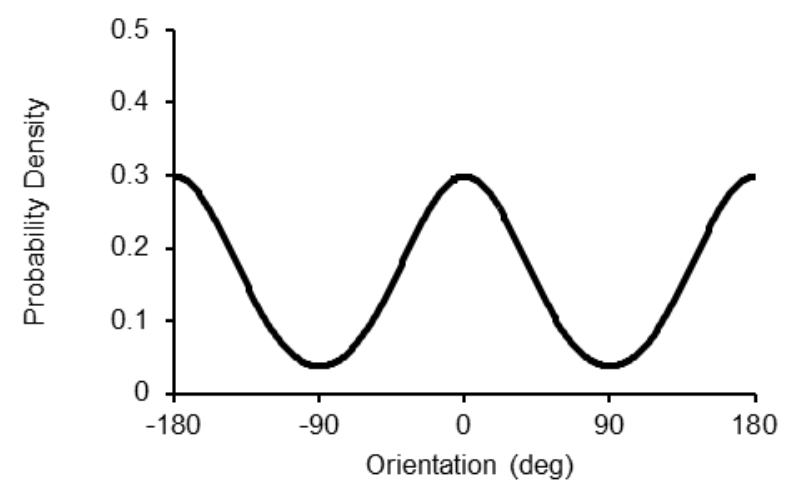

$\mathrm{C}$

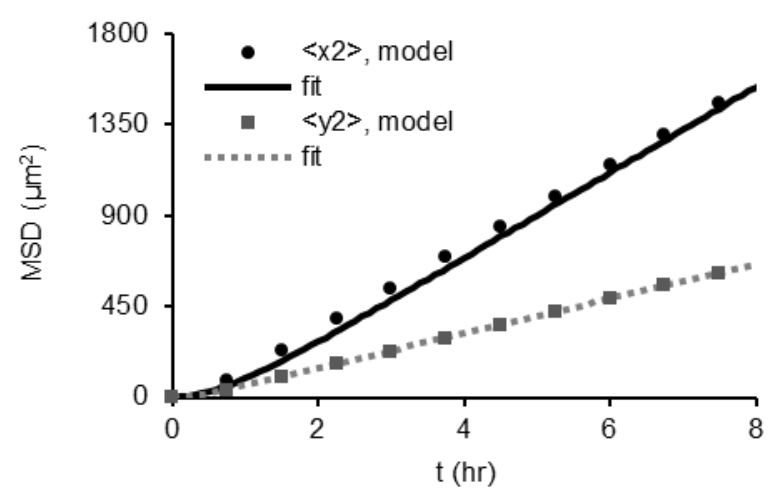

B

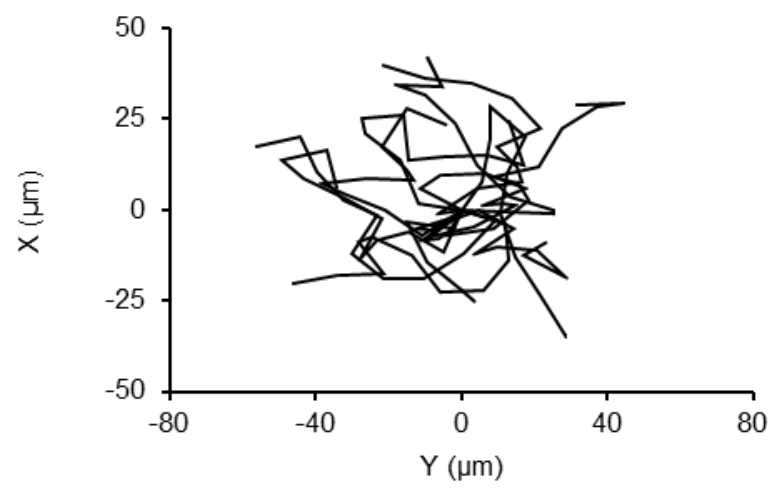

$\mathrm{D}$

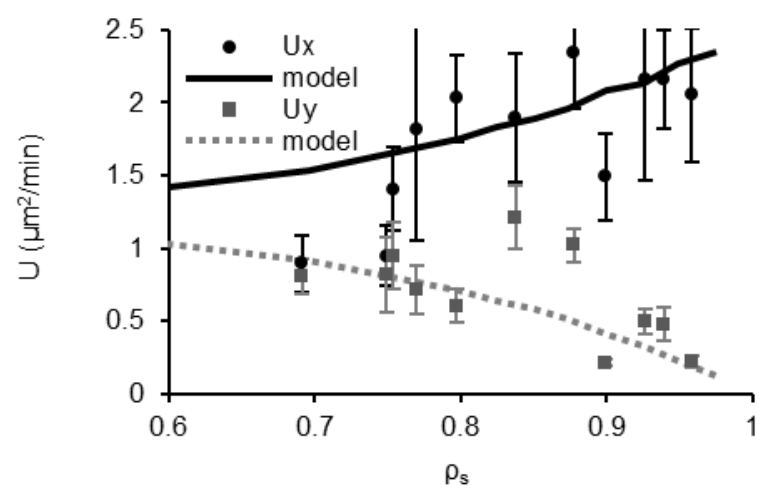

Figure 4.7. Cell migration in response to structural guidance cues. Dickinson et al. quantified the strength of alignment of fibroblasts and tracked their migration in collagen gels with varying strengths of collagen fiber alignment. The study reported random migration coefficients for motion of fibroblasts along the fiber (x-axis, 0 degrees) and cross-fiber (y-axis, 90 degrees) axes. We tested if fibroblast migration could be simulated as a series of displacements of magnitude $d$ and frequency $f$, where the orientation of each displacement was determined by randomly sampling the cell orientation distribution corresponding to a given strength of cell alignment. A. For a given strength of cell alignment $\left(\rho_{s}\right)$, we computed the expected cell orientation distribution. In this example, $\rho_{s}=0.8$. B. Migration tracks were simulated by displacing cells $d=17 \mu m$ every $f^{-1}=1 \mathrm{hr}$ along an angle determined by randomly sampling the cell orientation distribution at every step. C. Mean squared displacement (MSD) curves were calculated and fit to the persistent random walk model of cell migration in order to calculate random migration coefficients. D. The model predicted a relationship between the random migration coefficients $\left(U_{x}\right.$ and $\left.U_{y}\right)$ and cell mean vector length in good agreement with the experimental data. 
Aligned Deposition

A

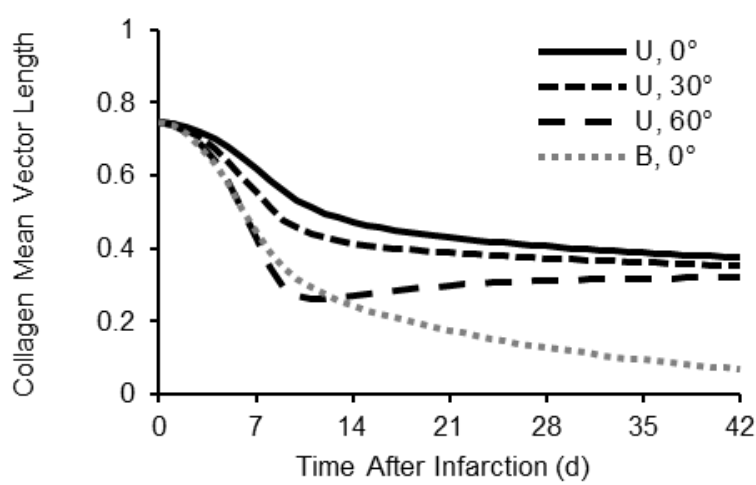

$\mathrm{C}$

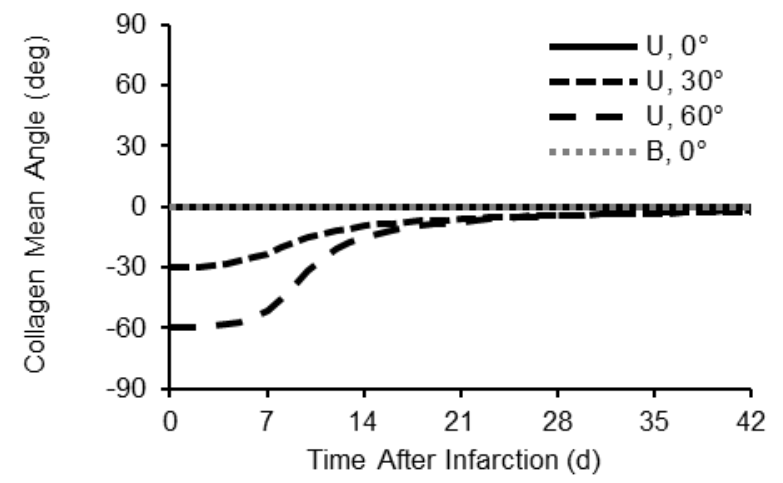

$\mathrm{E}$

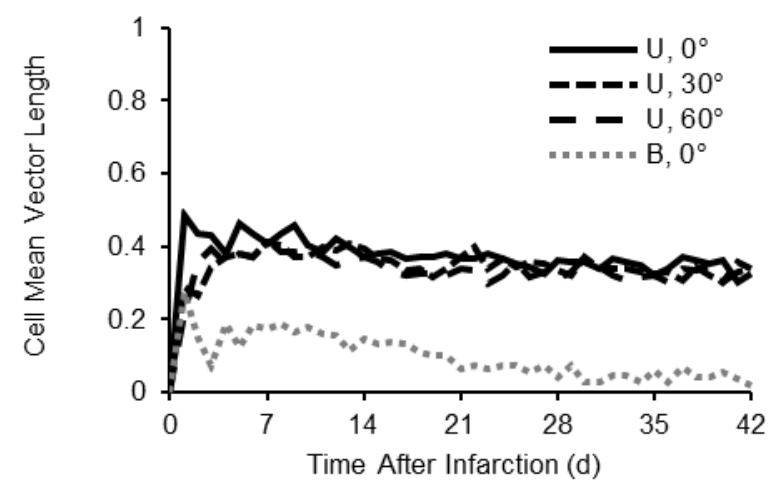

B

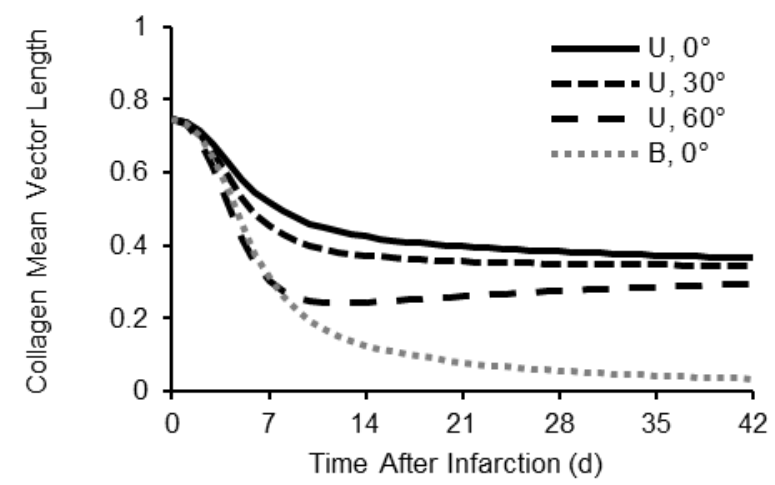

$\mathrm{D}$

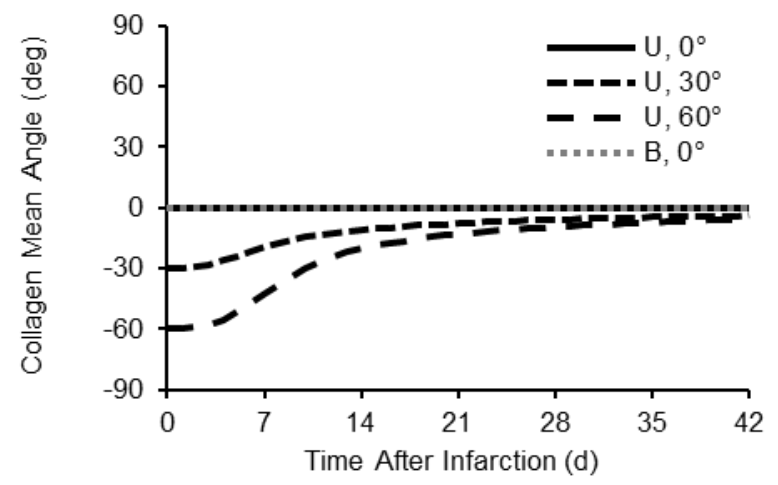

$\mathrm{F}$

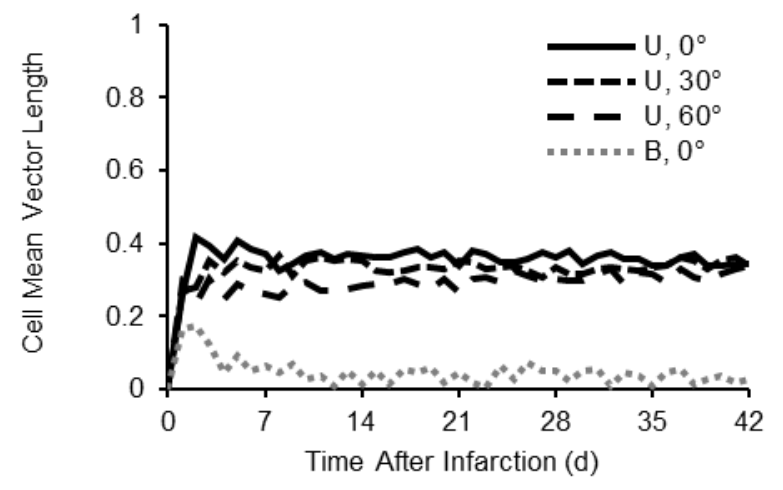

Figure 4.8. Revised agent-based model of infarct healing with collagen alignment allowed via aligned deposition only. After updating the agent-based model with equations and parameters based on in vitro data, the predictions of cell mean vector length, collagen mean vector length, and collagen mean angle were similar to the predictions of the prior model. 


\section{Rotation}

A

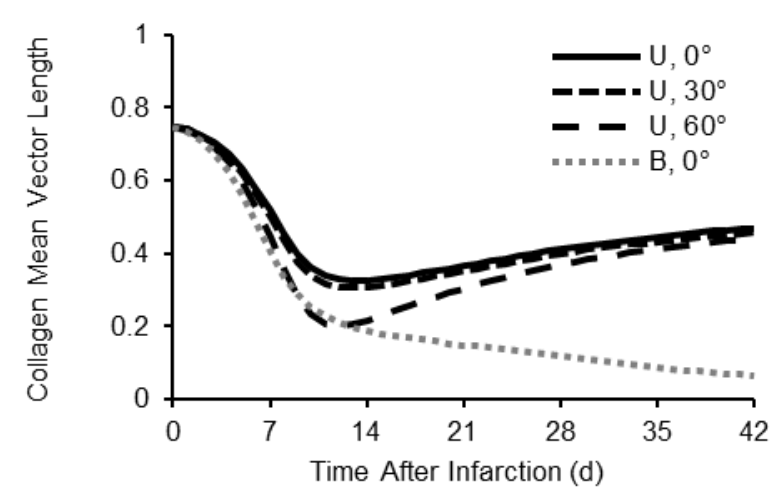

$\mathrm{C}$

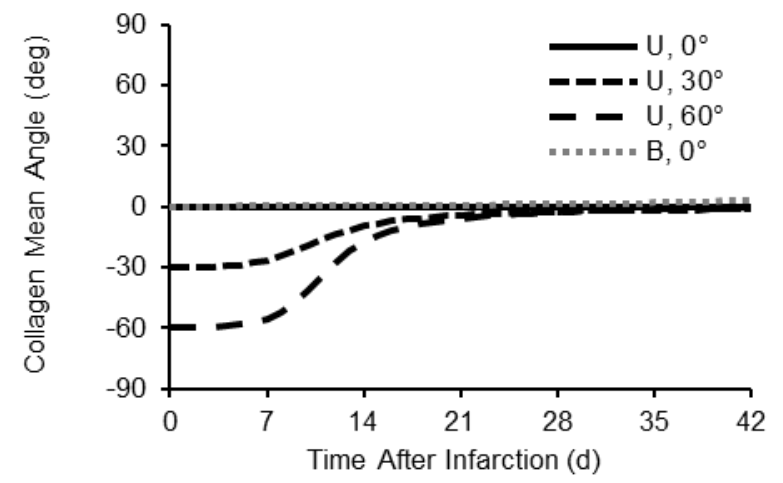

$\mathrm{E}$

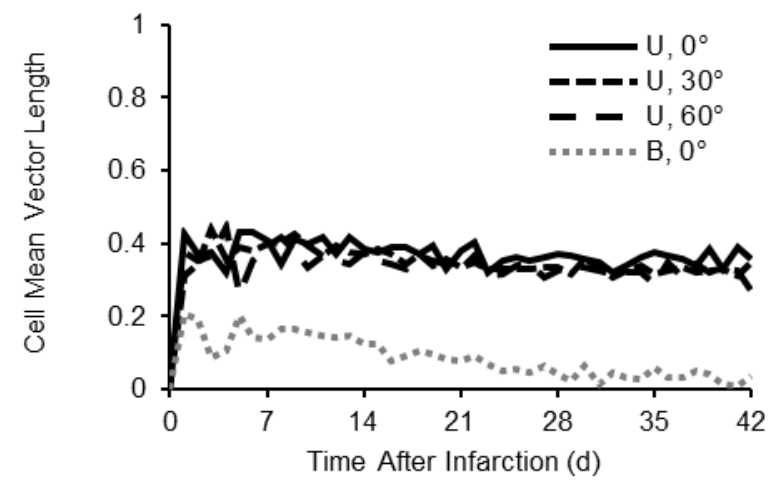

B

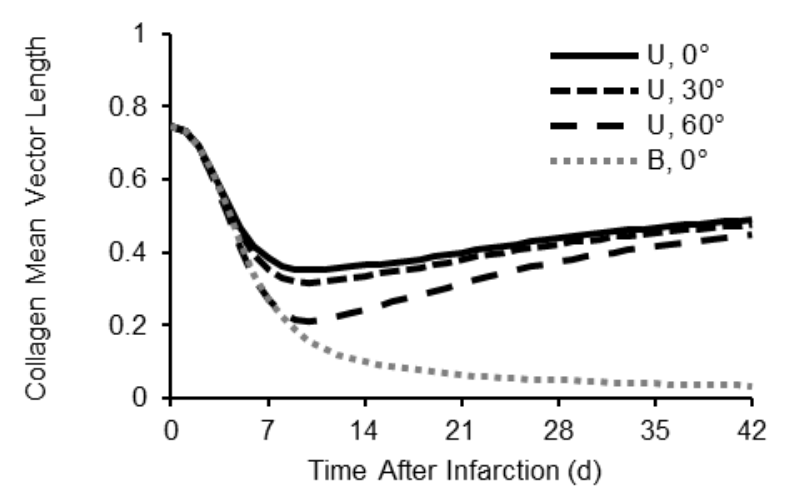

$\mathrm{D}$

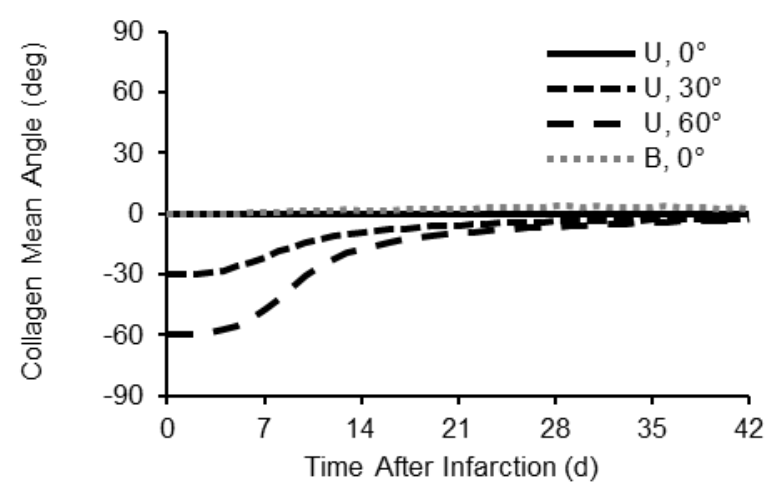

$\mathrm{F}$

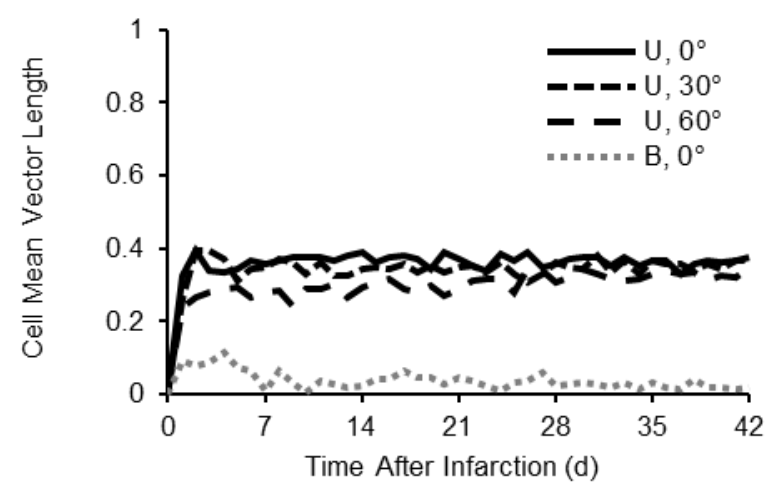

Figure 4.9. Revised agent-based model of infarct healing with collagen alignment allowed via rotation only. After updating the agent-based model with equations and parameters based on in vitro data, the predictions of cell mean vector length, collagen mean vector length, and collagen mean angle were similar to the predictions of the prior model. 


\subsection{REFERENCES}

Ahmed WW, Wolfram T, Goldyn AM, Bruellhoff K, Rioja BA, Möller M, Spatz JP, Saif TA, Groll J \& Kemkemer R (2010). Myoblast morphology and organization on biochemically micro-patterned hydrogel coatings under cyclic mechanical strain. Biomaterials 31, 250258.

Clark P, Connolly P, Curtis ASG, Dow JAT \& Wilkinson CDW (1990). Topographical control of cell behaviour: II. multiple grooved substrata. Development 108, 635-644.

Dickinson RB, Guido S \& Tranquillo RT (1994). Biased cell migration of fibroblasts exhibiting contact guidance in oriented collagen gels. Annals of Biomedical Engineering 22, 342-356.

Fomovsky GM, Rouillard AD \& Holmes JW (2012). Regional mechanics determine collagen fiber structure in healing myocardial infarcts. Journal of Molecular and Cellular Cardiology 52, 1083-1090.

Groh A \& Wagner M (2011). Biased three-dimensional cell migration and collagen matrix modification. Mathematical Biosciences 231, 105-119.

Henshaw DR, Attia E, Bhargava M \& Hannafin JA (2006). Canine ACL fibroblast integrin expression and cell alignment in response to cyclic tensile strain in three-dimensional collagen gels. Journal of Orthopaedic Research 24, 481-490.

Kang J, Steward RL, Kim Y, Schwartz RS, LeDuc PR \& Puskar KM (2011). Response of an actin filament network model under cyclic stretching through a coarse grained Monte Carlo approach. Journal of Theoretical Biology 274, 109-119.

Kaunas R \& Hsu H-J (2009). A kinematic model of stretch-induced stress fiber turnover and reorientation. Journal of Theoretical Biology 257, 320-330.

Kaunas R, Nguyen P, Usami S \& Chien S (2005). Cooperative effects of Rho and mechanical stretch on stress fiber organization. Proceedings of the National Academy of Sciences 102, $15895-15900$.

Kim D-H, Han K, Gupta K, Kwon KW, Suh K-Y \& Levchenko A (2009). Mechanosensitivity of fibroblast cell shape and movement to anisotropic substratum topography gradients. Biomaterials 30, 5433-5444.

Knapp DM, Helou EF \& Tranquillo RT (1999). A fibrin or collagen gel assay for tissue cell chemotaxis: assessment of fibroblast chemotaxis to GRGDSP. Experimental Cell Research 247, 543-553.

Lee EJ, Holmes JW \& Costa KD (2008). Remodeling of engineered tissue anisotropy in response to altered loading conditions. Annals of Biomedical Engineering 36, 1322-1334. 
Li J, Zhu L, Zhang M \& Lin F (2012). Microfluidic device for studying cell migration in single or co-existing chemical gradients and electric fields. Biomicrofluidics 6, 024121.

Lo C-M, Wang H-B, Dembo M \& Wang Y (2000). Cell movement is guided by the rigidity of the substrate. Biophysical Journal 79, 144-152.

Loesberg WA, Te Riet J, Van Delft FCMJM, Schön P, Figdor CG, Speller S, Van Loon JJWA, Walboomers XF \& Jansen JA (2007). The threshold at which substrate nanogroove dimensions may influence fibroblast alignment and adhesion. Biomaterials 28, 3944-3951.

Loesberg WA, Walboomers XF, Van Loon JJWA \& Jansen JA (2005). The effect of combined cyclic mechanical stretching and microgrooved surface topography on the behavior of fibroblasts. Journal of Biomedical Materials Research Part A 75, 723-732.

McDougall S, Dallon J, Sherratt J \& Maini P (2006). Fibroblast migration and collagen deposition during dermal wound healing: mathematical modelling and clinical implications. Philosophical Transactions of the Royal Society A: Mathematical, Physical and Engineering Sciences 364, 1385-1405.

Melvin AT, Welf ES, Wang Y, Irvine DJ \& Haugh JM (2011). In chemotaxing fibroblasts, both high-fidelity and weakly biased cell movements track the localization of PI3K signaling. Biophysical Journal 100, 1893-1901.

Neidlinger-Wilke C, Grood ES, Wang J H-C, Brand RA \& Claes L (2001). Cell alignment is induced by cyclic changes in cell length: studies of cells grown in cyclically stretched substrates. Journal of Orthopaedic Research 19, 286-293.

Pang Y, Wang X, Lee D \& Greisler HP (2011). Dynamic quantitative visualization of single cell alignment and migration and matrix remodeling in 3-D collagen hydrogels under mechanical force. Biomaterials 32, 3776-3783.

Pot SA, Liliensiek SJ, Myrna KE, Bentley E, Jester J V, Nealey PF \& Murphy CJ (2010). Nanoscale topography-induced modulation of fundamental cell behaviors of rabbit corneal keratocytes, fibroblasts, and myofibroblasts. Investigative Ophthalmology \& Visual Science 51, 1373-1381.

Prodanov L, Te Riet J, Lamers E, Domanski M, Luttge R, Van Loon JJWA, Jansen JA \& Walboomers XF (2010). The interaction between nanoscale surface features and mechanical loading and its effect on osteoblast-like cells behavior. Biomaterials 31, 7758-7765.

Rajnicek AM, Foubister LE \& McCaig CD (2007). Prioritising guidance cues: directional migration induced by substratum contours and electrical gradients is controlled by a rho/cdc42 switch. Developmental Biology 312, 448-460.

Smith JT, Tomfohr JK, Wells MC, Beebe TP, Kepler TB \& Reichert WM (2004). Measurement of cell migration on surface-bound fibronectin gradients. Langmuir 20, 8279-8286. 
Souders CA, Bowers SLK \& Baudino TA (2009). Cardiac fibroblast: the renaissance cell. Circulation Research 105, 1164-1176.

Sun J, Ding Y, Lin NJ, Zhou J, Ro H, Soles CL, Cicerone MT \& Lin-Gibson S (2010). Exploring cellular contact guidance using gradient nanogratings. Biomacromolecules 11, 3067-3072.

Sutherland J, Denyer M \& Britland S (2005). Contact guidance in human dermal fibroblasts is modulated by population pressure. Journal of Anatomy 206, 581-587.

Wagle MA \& Tranquillo RT (2000). A self-consistent cell flux expression for simultaneous chemotaxis and contact guidance in tissues. Journal of Mathematical Biology 41, 315-330.

Wang F (2009). The signaling mechanisms underlying cell polarity and chemotaxis. Cold Spring Harbor Perspectives in Biology 1, a002980.

Wang JH-C \& Grood ES (2000). The strain magnitude and contact guidance determine orientation response of fibroblasts to cyclic substrate strains. Connective Tissue Research 41, 29-36.

Wang JH-C, Yang G \& Li Z (2005). Controlling cell responses to cyclic mechanical stretching. Annals of Biomedical Engineering 33, 337-342.

Wang JH-C, Yang G, Li Z \& Shen W (2004). Fibroblast responses to cyclic mechanical stretching depend on cell orientation to the stretching direction. Journal of Biomechanics 37, 573-576.

Weis SM, Zimmerman SD, Shah M, Covell JW, Omens JH, Ross J, Dalton N, Jones Y, Reed CC, Iozzo R V \& McCulloch AD (2005). A role for decorin in the remodeling of myocardial infarction. Matrix Biology 24, 313-324.

Zhao M (2009). Electrical fields in wound healing-an overriding signal that directs cell migration. Seminars in Cell \& Developmental Biology 20, 674-682. 


\section{CHAPTER 5: Mechanical Regulation of Fibroblast Migration in a Collagen-Fibrin Wound Model}

\subsection{INTRODUCTION}

Many in vitro studies have shown that fibroblast behaviors such as proliferation (Berry et al., 2003), matrix synthesis (Atance et al., 2004), matrix degradation (Tyagi et al., 1998), and alignment (Katsumi et al., 2002; Lee et al., 2008; Pang et al., 2011) are sensitive to mechanical stimuli. Our analysis from Chapter 2 suggested that fibroblast alignment is sensitive to mechanical guidance cues during infarct healing, which simultaneously exposes fibroblasts to competing structural and chemical guidance cues. Little is known about the effect of mechanical guidance cues on fibroblast migration in such a wound environment. In Chapter 4, we formulated a quantitative approach to predicting fibroblast alignment and migration in response to mechanical, structural, and simultaneous mechanical and structural guidance cues. However, the data supporting the method of predicting fibroblast migration was limited to migration in response to an isolated structural guidance cue (Dickinson et al., 1994).

Inspired by Grinnell et al. (2006) who used nested collagen gels to study fibroblast migration, we developed a wound healing model using a three-dimensional collagen-fibrin gel composite that would allow us to address questions about the interaction of guidance cues during wound healing in a controlled, readily observable setting. Our goal was to test the hypothesis that anisotropic mechanical boundary conditions increase the directionality and speed of migration of cardiac fibroblasts into a model fibrin wound. We also tested if the response of fibroblasts to the 
anisotropic mechanical boundary conditions could be explained by strain-induced alignment of the wound matrix. Finally, we demonstrated that our approach to predicting the migration behavior of fibroblasts accurately captured the response of fibroblasts to the combinations of guidance cues encountered in our experimental wound healing model.

\subsection{MATERIALS AND METHODS}

The aim of this work was to test whether anisotropic mechanical boundary conditions significantly affect the directionality and speed of migration of cardiac fibroblasts into a model wound. Fibroblast-populated collagen gels were cast in square molds. An incision made in the center of each gel was filled with fibrin to simulate a wound. Uniaxial mechanical restraint was applied to the gels along the short or long axis of the fibrin "wound". (These cases will be referred to as "short axis restraint" and "long axis restraint".) After two days of culture, cell nuclei were fluorescently imaged. The distribution of cells that migrated into the incision was fit to the diffusion equation in order to compute the apparent diffusion coefficient as a measure of the rate of infiltration of fibroblasts. Wound remodeling strains were measured from zero to two days after creating the wounds in order to quantify the mechanical conditions within the wound. Fiber alignment within the fibrin wound matrix was quantified at zero and two days after wounding in order to investigate the role fiber alignment may play in mediating any mechanical effect on fibroblast migration. Migration tracks of individual cells in the wound region were measured one day after wounding and a computational model of fibroblast migration was used to infer what guidance cues, if any, the fibroblasts must have sensed in order to explain the observed migration tracks. 


\section{Adult Cardiac Fibroblast Isolation and Culture}

Caridac fibroblasts were isolated and cultured using previously published methods(Thomopoulos et al., 2005). Sprague-Dawley rats (six weeks old, $180 \mathrm{~g}$ ) were euthanized by $\mathrm{CO}_{2}$ narcosis. The chest was opened, the inferior vena cava and superior vena cava were transected, and the heart was perfused with ice cold sterile Krebs-Henseleit buffer (KHB) through a syringe needle inserted retrograde into the aorta. The heart was then transected to separate the ventricles from the atria, the ventricles were transferred to a solution of ice cold sterile KHB and transported to a sterile cell culture hood. The ventricles were placed in a dish containing sterile KHB warmed to $37 \mathrm{C}$ and minced into pieces approximately $1-3 \mathrm{~mm}$ in size. Care was taken to remove valve tissue, which appeared white. The minced cardiac tissue was transferred to a $50 \mathrm{ml}$ tube, washed four times with sterile, warm KHB, and then digested with Liberase Blendzyme 3 (Roche). Six consecutive 15 min digestions were performed with $10 \mathrm{ml}$ aliquots of the Liberase solution with gentle agitation in a cell culture incubator at $37{ }^{\circ} \mathrm{C}$ and $5 \% \mathrm{CO}_{2}$. After each digestion, the solution was vortexed for $30 \mathrm{sec}$ at $70 \%$ intensity. The supernatant was removed and centrifuged for $10 \mathrm{~min}$ at $400 \mathrm{~g}$ (except the supernatant from the first digestion was always discarded). The cell pellet was re-suspended in $20 \mathrm{ml}$ DMEM supplemented with $10 \%$ fetal bovine serum, 100 $\mathrm{U} / \mathrm{ml}$ penicillin, $100 \mathrm{~g} / \mathrm{ml}$ streptomycin, and $2 \mathrm{ng} / \mathrm{ml}$ amphotericin $\mathrm{B}$, and the cell suspension was transferred to a $75 \mathrm{~cm}^{2}$ cell culture flask. In order to remove non-fibroblast cells, after 4 hours the cell culture media was removed from the flasks, the flasks were rinsed with $10 \mathrm{ml}$ PBS, and $15 \mathrm{ml}$ of fresh cell culture media was added to the flasks. 
Cardiac fibroblasts were maintained in DMEM supplemented with 10\% fetal bovine serum, 100 $\mathrm{U} / \mathrm{ml}$ penicillin, $100 \mathrm{~g} / \mathrm{ml}$ streptomycin, and $2 \mathrm{ng} / \mathrm{ml}$ amphotericin $\mathrm{B}$, which will be referred to as cell culture media (CCM). Cell culture flasks were kept in an incubator at $37{ }^{\circ} \mathrm{C}$ and $5 \% \mathrm{CO}_{2}$. First passage was 4 days after isolation. Cells were re-plated at approximately $12,500 \mathrm{cells} / \mathrm{cm}^{2}$, cultured for two more days, and passaged a second time for use in the collagen-fibrin gel experiments. In order to passage the cells, the culture flasks were rinsed with PBS, incubated with trypsin-EDTA for $4 \mathrm{~min}$, and the detached cells were suspended in CCM. The suspension was centrifuged at $400 \mathrm{~g}$ for $10 \mathrm{~min}$, the cell pellet was re-suspended in CCM, cell concentration was quantified with a hemocytometer, and the appropriate volume of cells was added to $150 \mathrm{~cm}^{2}$ flasks with CCM to reach a total volume of $30 \mathrm{ml}$.

\section{Fabrication of Fibroblast-Populated Collagen-Fibrin Hydrogels}

Fibroblast-populated collagen gels were created by mixing 0.2 M HEPES (pH 9), 10x MEM, 3 $\mathrm{mg} / \mathrm{ml}$ type I bovine collagen (PureCol, Advanced Biomatrix), and cells suspended in CCM at a ratio of 1:1:8:2 (Thomopoulos et al., 2005). Ice cold HEPES, MEM, and collagen were quickly mixed in a $50 \mathrm{ml}$ tube and then kept ice cold to prevent the collagen from polymerizing before the cell suspension was ready. After the cell suspension was added, the collagen mixture was incubated with gentle agitation for $15 \mathrm{~min}$, thoroughly mixed by pipetting with a $10 \mathrm{ml}$ syringe, and then $2.5 \mathrm{ml}$ aliquots were distributed in a 6 well plate that contained porous, polyethylene molds. Once the collagen mixture was spread over the entire mold, plastic rings were placed on top of the molds to prevent them from floating. The 6 well plate was then incubated for 2 hours

to polymerize the collagen solution. The molds were then released from the bottoms of the 
wells, rinsed with PBS, and suspended in $2.5 \mathrm{ml} \mathrm{CCM}$ per well. The final result was a set of square fibroblast-populated collagen gels, equibiaxially restrained via attachment to the floating polyethylene mold. The collagen gels had final concentrations of $2 \mathrm{mg} / \mathrm{ml}$ collagen and 200,000 cells/ml.

The polyethylene molds were discs $3.25 \mathrm{~cm}$ in diameter and $3 \mathrm{~mm}$ in thickness with a 1.5 by 1.5 $\mathrm{cm}$ square cut out of the center. The molds were pre-treated with concentrated sulfuric acid for 3 days to increase their hydrophilicity (which improved absorption of the collagen mixture into the pores of the mold), dialyzed for 3 days with DI water to extract all sulfuric acid, dried, and then sterilized in an autoclave (Kolodney \& Wysolmerski, 1992). The 6 well plates were pre-coated with BSA to prevent the gels from sticking to the polystyrene surface. Plates were incubated overnight with sterile $2 \%$ BSA, rinsed twice with cold PBS, and air-dried in a sterile cell culture hood.

After incubation for $24 \mathrm{hrs,}$ a fibrin "wound" was created in each collagen gel (Fig. 5.1). For each gel, media was removed from the well. A $5 \mathrm{~mm}$ slit was cut in the center of the gel using a razor blade. The gel was rinsed with PBS and transferred to a new well. PBS was removed, and care was taken to extract as much free fluid as possible with a micropipette. $1 \mathrm{ml}$ of a fibrinogen mixture was poured under and over the collagen gel to fill in the slit. The polyethylene mold was again locked in place with a plastic ring and the gel was incubated for 1 hour to polymerize the fibrinogen mixture into a fibrin gel. The gel was then rinsed with PBS and suspended in 2.5 ml CCM supplemented with $100 \mathrm{ng} / \mathrm{ml}$ PDGF-BB, $100 \mathrm{ng} / \mathrm{ml} \mathrm{IGF-1,} \mathrm{and} 50 \mu \mathrm{g} / \mathrm{ml}$ ascorbic acid to promote fibroblast migration and collagen secretion (Shreiber et al., 2001; Diaz-Araya et al., 
2003; Williams et al., 2006). Finally, a uniaxial mechanical boundary condition was imposed by cutting two opposing sides of the gel free from the polyethylene mold. Uniaxial mechanical restraint acted along either the short or long axis of the fibrin wound.

The fibrinogen mixture was prepared by mixing $0.5 \mathrm{ml}$ of $6 \mathrm{mg} / \mathrm{ml}$ fibrinogen, $0.5 \mathrm{ml}$ of CCM, 1 $\mu \mathrm{l}$ of $1 \mathrm{M} \mathrm{CaCl}_{2}, 1 \mu \mathrm{l}$ of $100 \mu \mathrm{g} / \mathrm{ml}$ aminocaproic acid, and $2.5 \mu \mathrm{l}$ of $100 \mathrm{U} / \mathrm{ml}$ thrombin (Grassl et al., 2003; Williams et al., 2006; Robinson et al., 2008). Thrombin was not added until just before adding the mixture to the collagen gels. For tracking the migration paths of individual cells, some fibrin gels were prepared with $5 \cdot 10^{5}$ particles/ml of $10 \mu \mathrm{m}$ latex microspheres in order to calculate the movement of cells relative to the surrounding matrix.

\section{Microscopy and Histology}

The collagen-fibrin gels were cultured for two days. For the gels with microspheres embedded in the fibrin gel, after one day, the fibrin wounds were imaged on a light microscope with a $4 \mathrm{X}$ objective every hour for a total of six hours. Phase contrast was used to image cells, while ordinary light was used to image microspheres. After two days, gels were fixed for 24 hours in 4\% para-formaldehyde, cell nuclei were stained with DAPI, and the fibrin wounds were imaged at $4 \mathrm{X}$ on a fluorescence microscope. In the fluorescence images, the higher level of background fluorescence from collagen clearly delineated the interface between the collagen and fibrin gels. Some gels were processed for paraffin-embedding, cut into $10 \mu \mathrm{m}$ sections, stained with eosin, and imaged on a confocal microscope. Eosin is fluorescent over a broad range of wavelengths (De Rossi et al., 2007). Good signal was acquired with excitation at $543 \mathrm{~nm}$ and acquisition at 
$590 / 50 \mathrm{~nm}$. We selected fields located along the short axis of the wound that were devoid of cells and imaged fibrin fiber structures with a $60 \mathrm{X}$ objective.

\section{Quantification of Cell Migration Characteristics}

For the analysis of cell distributions after two days of migration, the border of the wound region was traced and fit to an ellipse, and the coordinates of the DAPI stained cell nuclei were automatically acquired with a custom image processing algorithm implemented in Matlab that identified intensity peaks after background subtraction and noise filtering (Fig. 5.2). In a subset of images, coordinates of cell nuclei were measured manually in order to verify the accuracy of the automated approach. Only cells near the middle of the long axis of the wound were analyzed in order to restrict the analysis to cells that entered from the sides of the wound. The region of interest had length $\mathrm{L}$ that spanned the short axis of the wound and width $\mathrm{W}$ that spanned the middle third of the long axis of the wound. We calculated the cumulative number of cells that entered from the sides of the wounds as a function of distance from the edge of the wound to the center of the wound. The cumulative number of cells was normalized by the remote cell concentration $\left(\mathrm{C}_{\mathrm{R}}\right)$ in the collagen gel $(500 \mu \mathrm{m}$ away from the wound border) multiplied by the area of the region of interest $(\mathrm{L} \cdot \mathrm{W})$. Distance was normalized by the short axis length of the wound. We took advantage of symmetry about the long axis of the wound and averaged data from both sides of the wound into a single plot. In order to obtain a measure of the rate of infiltration of the population of fibroblasts into the wound, apparent diffusion coefficients were computed by fitting the cumulative cell distribution of each wound to the solution of the 
diffusion equation for 1D unsteady diffusion into a space with symmetric, constant concentration boundary conditions:

$\frac{\partial C}{\partial t}=D \frac{\partial^{2} C}{\partial x^{2}}$

$\frac{N(x, t)}{C_{o} W L}=\frac{x}{L}-\sum_{n_{o d d}}^{\infty} \frac{4 e^{n^{2} \pi^{2} \frac{D t}{L^{2}}}}{n^{2} \pi^{2}}\left[1-\cos \left(n \pi \frac{x}{L}\right)\right]$

$C$-cell concentration, $t$ - time, $D$ - diffusion coefficient, $x$-position from wound edge along short axis, $N$ - cell number, $C_{o}-$ cell concentration at $x=0$ and $x=L, W-$ width of region of interest, $L$ - length of region of interest (short axis length)

For the analysis of cell migration tracks, coordinates of cells and microspheres were acquired manually from the time lapse images using ImageJ. Only cells within the center third of the long axis of the wound were analyzed. For each one hour time interval and each tracked fibroblast, we computed the displacement of the fibroblast relative to the centroid of nearby microspheres (within a $500 \mu \mathrm{m}$ radius). Migration tracks were calculated as the cumulative sum of these displacements over time. For each 1, 2, 3, 4, and 5 hour time interval and each tracked fibroblast, the squared displacement (square of the straight line distance from start point to end point) and squared path length (square of the cumulative distance from start point to end point through all intermediate points) were calculated and then averaged to generate mean squared displacement $\left(\left\langle\mathrm{d}^{2}\right\rangle,\left\langle\mathrm{x}^{2}\right\rangle,\left\langle\mathrm{y}^{2}\right\rangle\right)$ versus time curves and mean squared path length $\left(\left\langle\mathrm{d}_{\mathrm{p}}{ }^{2}\right\rangle,\left\langle\mathrm{x}_{\mathrm{p}}{ }^{2}\right\rangle\right.$, $\left.\left\langle\mathrm{y}_{\mathrm{p}}{ }^{2}\right\rangle\right)$ versus time curves. These calculations were performed on the full two-dimensional cell motions (d) and on the one-dimensional projections of the motions onto the short (x) and long (y) axes as in the study by Dickinson et al. (1994). For each one hour time interval and each tracked fibroblast, the apparent orientation and apparent speed of the cell were calculated as the angle of 
the cell displacement vector and as the magnitude of the displacement vector divided by the time step (one hour), respectively. We generated histograms of apparent orientations and plotted the average apparent speed for each apparent orientation bin.

\section{Quantification of Fibrin Wound Deformation and Matrix Structure}

In order to assess how the application of uniaxial mechanical restraint at the edges of the collagen gel affected the mechanical conditions within the fibrin wound, the short and long axis dimensions of the wound were measured at the beginning and end of the experiment, and remodeling strains were computed. We defined a metric of mechanical (or strain) anisotropy $(\Delta \mathrm{E})$ as the short axis remodeling strain $\left(\mathrm{E}_{\mathrm{S}}\right)$ minus the long axis remodeling strain $\left(\mathrm{E}_{\mathrm{L}}\right)($ Eqn. 5.3-5). Positive values indicated greater stretch or less compaction along the short axis than along the long axis. Negative values indicated greater stretch or less compaction along the long axis than along the short axis. 
$\Delta E=E_{S}-E_{L}$

$E_{S}=\frac{\left(L_{S, 2 d}-L_{S, 0 d}\right)}{L_{S, 0 d}}$

$E_{L}=\frac{\left(L_{L, 2 d}-L_{L, 0 d}\right)}{L_{L, 0 d}}$

$\Delta E$ - mechanical or strain anisotropy, $E_{S}$ - short axis remodeling strain, $L_{S, 2 d}$ - short axis length at Day 2, $L_{S, 0 d}$ - short axis length at Day 0, $E_{L}$ - long axis remodeling strain, $L_{L, 2 d}-$ long axis length at Day 2, $L_{L, 0 d}$ - long axis length at Day 0

In order to test if the wound deformation caused alignment of the fiber structure within the wound, the strength of alignment of the fibrin matrix was quantified at the beginning and end of the experiment. We used Fiber3, an automated gradient detection algorithm, to measure fiber orientation within sub-regions of each confocal image (Karlon et al., 1998, 1999). We calculated the mean angle (MA) and mean vector length (MVL) of the fiber orientations of each gel (Eqn. 5.6-7) (Mardia \& Jupp, 2000) and generated average fiber orientation histograms. We used the mean cosine of doubled fiber angles $(<\cos (2 \theta)>)$ as a metric of structural anisotropy, which has a range from -1 to 1 , where positive values indicated fiber alignment along the short axis and negative values indicated fiber alignment along the long axis (Dickinson et al., 1994; Thomopoulos et al., 2005). In order to check if the structures measured at the end of the experiment could be explained by deformation of the wounds, we used the measured remodeling strains to apply an affine deformation to the initial fiber distribution and computed the structural anisotropy $(<\cos (2 \theta)>)$ of the deformed fiber distribution. 
$M V L=\sqrt{\left(\frac{\sum_{i=1}^{n} \cos 2 \theta_{i}}{n}\right)^{2}+\left(\frac{\sum_{i=1}^{n} \sin 2 \theta_{i}}{n}\right)^{2}}$

$M A=\frac{1}{2} \tan _{-\pi: \pi}^{-1}\left[\frac{\left(\frac{\sum_{i=1}^{n} \sin 2 \theta_{i}}{n}\right)}{\left(\frac{\sum_{i=1}^{n} \cos 2 \theta_{i}}{n}\right)}\right]$

$M V L$ - mean vector length, $\theta_{i}-\mathrm{i}^{\text {th }}$ angle out of population sample, $M A$ - mean angle, $\tan _{-\pi: \pi}^{-1}$ - inverse tangent resolved fully over the domain from $-\pi$ to $\pi$

\section{Computational Model of Fibroblast Migration}

In Chapter 4, we formulated an approach to predicting the migration behavior of cells in response to environmental guidance cues. We assumed that the influence of guidance cues on the orientation probability distribution of a cell could be described using wrapped normal probability density functions. We took advantage of the fact that wrapped normal distributions can be defined using vectors (the angle and magnitude of the vector define the mean angle and circular standard deviation of the wrapped normal probability density function) to formulate a vector averaging approach to predicting the combined influence of simultaneous guidance cues on cell orientation. From the resultant orientation probability distribution, we computed cell migration tracks by displacing simulated cells $d_{c} \mu \mathrm{m}$ every $f^{-1}$ min, where the orientation of each displacement was determined by randomly sampling the orientation distribution.

Here, we use the approach formulated in Chapter 4 in order to address the problem of inferring environmental guidance cues from measurements of cell migration behavior. First, we adapt the approach to handle unidirectional guidance cues, such as chemotactic gradients, which may be present in the fibrin wounds. Then we outline a strategy for inferring what guidance cues can 
explain a given set of measurements of cell migration. Because of our focus on migration in this chapter, we will discuss guidance cues in terms of their effect on the orientation of cell displacements, not on the orientation of whole cells, and avoid unnecessarily assuming that the two orientations are always perfectly correlated.

Guidance cues can be unidirectional or bidirectional. Unidirectional guidance cues, such as chemotactic or haptotactic gradients, cause cells to prefer a single direction of migration, whereas bidirectional guidance cues, such as uniaxial strain and aligned fibers, cause cells to prefer an axis of migration (with equal preference for either of the two anti-parallel directions along that axis). We assumed these migration biases could be described with displacement orientation probability distributions $(\varphi)$ (Eqn. 5.8-11). We used a single wrapped normal probability density function $\left(\varphi_{w n}\right)$ to represent the bias due to a unidirectional guidance cue. We used the average of two identical wrapped normal probability density functions offset by 180 degrees to represent the bias due to a bidirectional guidance cue.

$\varphi_{u}(\theta)=\varphi_{w n}\left(\theta: \rho_{u}, \bar{\theta}_{u}\right)$

$\varphi_{b}(\theta)=\frac{1}{2}\left[\varphi_{w n}\left(\theta: \rho_{b}, \bar{\theta}_{b}\right)+\varphi_{w n}\left(\theta: \rho_{b}, \bar{\theta}_{b}+\pi\right)\right]$

$\varphi_{w n}(\theta: \rho, \bar{\theta})=\frac{1}{\sigma \sqrt{2 \pi}} \sum_{k=-\infty}^{\infty} e^{-(\theta-\bar{\theta}+2 \pi k)^{2} /(2 \sigma)^{2}}$

$\sigma^{2}=-2 \ln \rho$

$\varphi_{u}-$ unidirectional displacement orientation probability density function, $\varphi_{b}-$ bidirectional displacement orientation probability density function, $\theta-$ displacement orientation, $\varphi_{w n}-$ wrapped normal probability density function, $\rho$ - mean vector length of the wrapped normal 
probability density function, $\bar{\theta}$ - mean angle of the wrapped normal probability density function, $\sigma-$ circular standard deviation of the wrapped normal probability density function

The wrapped normal distribution is defined by a mean angle $(\bar{\theta})$ and mean vector length $(\rho)$, which we used as common metrics of the orientation and strength of the migration bias (mean vector length can vary from 0 to 1 , where 0 indicates no alignment and 1 indicates perfect alignment) (Mardia \& Jupp, 2000). We used the mean angle and mean vector length to define vectors, which we called displacement probability vectors $(\vec{d})$, associated with each displacement orientation distribution (Eqn. 5.12-14). The bidirectional displacement orientation distribution was associated with two antiparallel vectors.

$\vec{d}_{u}=\rho_{u} \cos \left(\bar{\theta}_{u}\right) \vec{e}_{x}+\rho_{u} \sin \left(\bar{\theta}_{u}\right) \vec{e}_{y}$

$\vec{d}_{b 1}=\rho_{b} \cos \left(\bar{\theta}_{b}\right) \vec{e}_{x}+\rho_{b} \sin \left(\bar{\theta}_{b}\right) \vec{e}_{y}$

$\vec{d}_{b 2}=-\vec{d}_{b 1}$

$\vec{d}$ - displacement probability vector, $\rho$ - mean vector length of the wrapped normal probability density function describing the displacement orientation distribution, $\bar{\theta}$ - mean angle of the wrapped normal probability density function describing the displacement orientation distribution

We assumed that when a unidirectional and bidirectional guidance cue act simultaneously, the resultant displacement orientation distribution $\left(\varphi_{r}\right)$ can be determined by 1) computing a weighted sum of the unidirectional displacement probability vector with the first bidirectional displacement probability vector, 2) computing a weighted sum of the unidirectional displacement probability vector with the second bidirectional displacement probability vector, and 3) 
averaging the wrapped normal probability density functions associated with each of the resultant displacement probability vectors (Eqn. 5.15-20).

$\vec{d}_{r 1}=\omega_{u} \vec{d}_{u}+\omega_{b} \vec{d}_{b 1}$

$\vec{d}_{r 2}=\omega_{u} \vec{d}_{u}+\omega_{b} \vec{d}_{b 2}$

$\omega_{u}+\omega_{b}=1$

$\rho_{r i}=\left\|\vec{d}_{r i}\right\|, \quad i=1,2$

$\bar{\theta}_{r i}=\angle \vec{d}_{r i}, \quad i=1,2$

$\varphi_{r}(\theta)=\frac{1}{2}\left[\varphi_{w n}\left(\theta: \rho_{r 1}, \bar{\theta}_{r 1}\right)+\varphi_{w n}\left(\theta: \rho_{r 2}, \bar{\theta}_{r 2}\right)\right]$

$\vec{d}_{r 1}$ and $\vec{d}_{r 2}-$ resultant displacement probability vectors, $\omega_{i}-$ normalized weight factor of guidance cue i, $\rho_{r 1}$ and $\rho_{r 2}-$ mean vector lengths of the wrapped normal probability density functions describing the resultant displacement orientation distribution, $\bar{\theta}_{r 1}$ and $\bar{\theta}_{r 2}-$ mean angles of the wrapped normal probability density functions describing the resultant displacement orientation distribution

We simulated fibroblast migration as a biased random walk. We chose a displacement frequency $f=1 \mathrm{~min}^{-1}$ and displacement magnitude $d_{c} \sim 2 \mu \mathrm{m}$ (to be determined), and computed cell displacements for a sequence of orientations randomly sampled from the resultant displacement orientation distribution $\left(\varphi_{r}\right)$. We simulated the migration of 50,000 fibroblasts and replicated our experimental protocol by saving cell positions every hour for five hours of simulated time.

We used the model of fibroblast migration to determine what combination of guidance cues, if any, gave rise to the migration behavior that we measured. We considered the possible existence 
of two guidance cues: a bidirectional guidance cue, due to mechanical and/or structural anisotropy, oriented along the axis of mechanical restraint $\left(\bar{\theta}_{b}=90^{\circ}\right.$ for long axis restraint and $\bar{\theta}_{b}=0^{\circ}$ for short axis restraint); and a unidirectional guidance cue, due to chemo- and/or haptotaxis, oriented toward the center of the wound $\left(\bar{\theta}_{u}=0^{\circ}\right)$ (Fig. 5.3a,b). As described above, we calculated the resultant displacement probability vectors $\vec{d}_{r 1}$ and $\vec{d}_{r 2}$ (Fig. 5.3a,b), calculated the resultant displacement orientation distribution $\varphi_{r}(\theta)$ (Fig. 5.3c,d), and then simulated migration tracks (Fig. 5.3e).

Our goal was to solve for the displacement magnitudes $\left(d_{c}\right)$ and resultant displacement orientation distributions $\left(\varphi_{r}\right)$ that best reproduced the migration tracks we observed for long axis restraint and short axis restraint. Three free model parameters - the displacement magnitude $\left(d_{c}\right)$, the weighted magnitude of the bidirectional displacement probability vector $\left(\omega_{b} \rho_{b}\right)$, and the weighted magnitude of the unidirectional displacement probability vector $\left(\omega_{u} \rho_{u}\right)$ - were determined by fitting model results to experimental data. A multi-parameter, multi-objective optimization problem was solved by minimizing a weighted sum of two objectives: 1) the normalized root mean squared difference between model and experiment of the apparent orientation histogram and 2) the normalized root mean squared difference between model and experiment of the average apparent speed versus apparent orientation curve.

Objective Function $=w \sqrt{\frac{\sum_{i=1}^{n}\left[F_{e}\left(\theta_{i}\right)-F_{m}\left(\theta_{i}\right)\right]^{2}}{\sum_{i=1}^{n}\left[F_{e}\left(\theta_{i}\right)\right]^{2}}}+(1-w) \sqrt{\frac{\sum_{i=1}^{n}\left[S_{e}\left(\theta_{i}\right)-S_{m}\left(\theta_{i}\right)\right]^{2}}{\sum_{i=1}^{n}\left[S_{e}\left(\theta_{i}\right)\right]^{2}}}$ $w$-weight factor, $n$ - number of orientation bins (36), $F\left(\theta_{i}\right)$ - fraction of cells aligned within $5^{\circ}$ of $\theta_{i}, S\left(\theta_{i}\right)$ - average apparent speed of cells aligned within $5^{\circ}$ of $\theta_{i}$, subscript $e-$ experimental data, subscript $m$ - model prediction 
We varied the weight factor from 0.01 to 0.99 and calculated the optimal solution (parameter set) for each weight factor by brute force. That is, we computed objective values over a fine mesh of parameter space and explicitly calculated the minimum of the weighted sum of objectives. A posteriori, we decided to average the optimal solutions because we observed tight clustering of the optimal solutions over the range of weight factors.

\section{Statistics}

Unpaired, two-tailed student's t-test was used to test if apparent diffusion coefficient ( $\left.D_{\text {app }}\right)$ differed significantly between the short axis restraint and long axis restraint groups. Onesample, two-tailed student's t-test was used to test if mechanical anisotropy $(\Delta \mathrm{E})$ and structural anisotropy $(<\cos (2 \theta)>)$ differed significantly from a hypothetical value of zero. A two-tailed test

of the t-statistic $t=m / S . E_{\cdot m}$, where $m$ is a regression slope and $S . E_{\cdot m}$ is the standard error of the slope, was used to test if the slope of the correlation between $D_{\text {app }}$ and $\Delta E$ differed significantly from a hypothetical value of zero. 


\subsection{RESULTS}

\section{Mechanical Environment within Fibrin Wounds}

We restrained the collagen gels along either the short axis or long axis of the fibrin wounds in order to create different mechanical environments within the wounds, which we characterized by measuring wound remodeling strains between zero and two days after creating the wounds. For the case of short axis restraint, the wound remodeling strains were 0.09 along the short axis and 0.29 along the long axis (Table 5.1). The mechanical anisotropy was $\Delta E=0.38 \pm 0.18$ $(p<0.05)$, suggesting the presence of a mechanical guidance cue. For the case of long axis restraint, the wound remodeling strains were -0.07 along the short axis and -0.10 along the long axis (Table 5.1). The mechanical anisotropy was $\Delta E=0.03 \pm 0.07$ ( $p=n . s$.$) , suggesting the$ absence of a mechanical guidance cue.

\section{Rate of Infiltration of Fibroblasts into Fibrin Wounds}

We measured the spatial distribution of fibroblasts within the fibrin matrix two days after creating the wounds in order to test if the rate at which fibroblasts entered the wounds depended on the mechanical boundary conditions. More fibroblasts travelled farther into the wounds along the short axis when mechanical restraint acted along the short axis than when mechanical restraint acted along the long axis. We quantified this observation by calculating the cumulative number of cells from the edge of the wound to the center of the wound and fit the cumulative distribution to the diffusion equation (Fig. 5.4a). The apparent diffusion coefficient $\left(D_{a p p}\right)$, 
which indicated the rate of infiltration along the short axis, was 2.6-fold greater when mechanical restraint acted along the short axis than when mechanical restraint acted along the long axis $(p<0.05)$ (Table 5.1). Across all gels, the apparent diffusion coefficient was positively correlated with mechanical anisotropy (Fig. 5.4b). Least squares linear regression yielded a slope of $760 \mu \mathrm{m}^{2} / \mathrm{hr}$ with a standard error of $240 \mu \mathrm{m}^{2} / \mathrm{hr}(p<0.05)$ and correlation coefficient of 0.17 .

\section{Fiber Alignment within Fibrin Wounds}

We measured the strength of alignment of fibrin fibers in the wound matrix in order to test if structural guidance cues formed as a result of the wound deformation. Eosin stained fibrin matrices were readily observed using confocal microscopy (Fig. 5.5a). Immediately after creating the wounds, fibrin fibers were slightly aligned along the long axis of the wound for both long axis restraint and short axis restraint (Fig. 5.5b). Two days after creating the wounds, fibrin fibers became slightly more aligned along the long axis of the wound for long axis restraint, whereas fibrin fibers became slightly aligned along the short axis of the wound for short axis restraint (Fig. 5.5b). We used the mean of the cosine of doubled fiber angles $(<\cos (2 \theta)>)$ to quantify structural anisotropy and plotted this metric against our measure of mechanical anisotropy $(\Delta E)$. Structural anisotropy was positively correlated with strain anisotropy, and the measurements agreed reasonably with the values predicted assuming affine deformation of the average initial fiber structure (Fig. 5.5c). For short axis restraint, the structural anisotropy was $\langle\cos (2 \theta)\rangle=0.11 \pm 0.06(p<0.05)$, suggesting the presence of a structural guidance cue 
(Table 5.1). For long axis restraint, the structural anisotropy was $\langle\cos (2 \theta)\rangle=0.35 \pm 0.19$ $(p<0.05)$, suggesting the presence of a structural guidance cue (Table 5.1).

\section{Migration Behavior of Fibroblasts in Fibrin Wounds}

We tracked the migration of individual fibroblasts in the fibrin wounds in order to determine if a difference in the direction or speed of fibroblast migration could explain why the apparent infiltration rate differed between groups (Fig. 5.6a,b). We parsed the migration tracks into apparent displacement vectors for each one hour time interval and created scatterplots of apparent speed versus apparent orientation (Fig. 5.7a,b). We calculated apparent orientation histograms, mean apparent speed versus apparent orientation curves, mean squared displacement versus time curves, and mean squared path length versus time curves in order to quantitatively summarize the migration behavior (Fig. 5.8). Fibroblasts migrated preferentially toward the center of the wound for both long axis restraint and short axis restraint (Fig. 5.8a). However, fibroblast migration was oriented more strongly along the short axis of the wound with short axis restraint than with long axis restraint (Fig. 5.8a). Apparent speeds were highest toward the center of the wound for both long axis restraint and short axis restraint (Fig. 5.8b). Apparent speeds along the short axis of the wound were similar for short axis restraint and long axis restraint (Fig. 5.8b). 


\section{Computational Model of Fibroblast Migration Guided by Environmental Cues}

We simulated fibroblast migration with a computational model in order to determine which guidance cues, if any, could explain the migration behavior we observed. For long axis restraint, we tested if the migration behavior could be explained by a structural guidance cue (bidirectional guidance cue oriented along the long axis of the wound due to fiber alignment) acting simultaneously with a chemical guidance cue (unidirectional cue oriented toward the center of the wound due to chemo- or haptotactic gradients). For short axis restraint, we tested if the migration behavior could be explained by a mechanical guidance cue (bidirectional guidance cue oriented along the short axis of the wound due to strain anisotropy) acting simultaneously with a chemical guidance cue.

For long axis restraint, the optimal fit to the migration data yielded a displacement magnitude of $d_{c}=2 \mu m$, and weighted magnitudes of the displacement probability vectors of $\omega_{b} \rho_{b}=0.02$ for the structural (bidirectional) guidance cue and $\omega_{u} \rho_{u}=0.08$ for the chemical (unidirectional) guidance cue (Table 5.2, Fig. 5.9a,b). For short axis restraint, the optimal fit to the migration data yielded a displacement magnitude of $d_{c}=1.7 \mu \mathrm{m}$, and weighted magnitudes of the displacement probability vectors of $\omega_{b} \rho_{b}=0.82$ for the mechanical (bidirectional) guidance cue and $\omega_{u} \rho_{u}=0.10$ for the chemical (unidirectional) guidance cue (Table 5.2, Fig. 5.9a,b). These parameter values prescribed a unimodal distribution of displacement orientations for long axis restraint and an asymmetric, bimodal distribution for short axis restraint (Fig. 5.9c,d). 
The computational model simultaneously reproduced the apparent orientation histogram, apparent speed versus apparent orientation curve, mean squared displacement curves, and mean squared path length curves for both long axis restraint and short axis restraint (Fig. 5.8). The model also reproduced the shape and scatter of the apparent speed versus apparent orientation data, except for the small subset of displacements with relatively high apparent speed (Fig. $5.7 \mathrm{c}, \mathrm{d})$. Similarly, the model reproduced the fibroblast migration tracks, except for the small subset of cells with relatively high net displacement (Fig. 5.6c,d).

\subsection{DISCUSSION}

Prior studies have shown that fibroblasts in collagen gels align and reorient collagen fibers when anisotropic mechanical boundary conditions are applied to the gels (Thomopoulos et al., 2005; Lee et al., 2008). We tested if anisotropic mechanical boundary conditions affect the directionality and speed of fibroblasts migrating into a three-dimensional model wound, which could simultaneously expose fibroblasts to mechanical, structural, and chemical guidance cues. We created fibrin "wounds" in fibroblast-populated collagen gels and applied uniaxial mechanical restraint at the edges of the collagen gels along the short or long axis of the fibrin wounds. We measured the migration of fibroblasts into the fibrin wounds and used a computational model to infer what guidance cues could explain the migration behavior that we observed. We found that the migration behavior could be explained as a response to mechanical and chemical guidance cues that biased the orientation probability distribution of cell displacements. 


\section{Mechanical Regulation of Fibroblast Migration during Wound Healing}

The rate of infiltration of fibroblasts along the short axis of the fibrin wounds was higher with short axis restraint than with long axis restraint. We considered two explanations of the higher rate of infiltration with short axis restraint: mechanical and/or structural cues could have guided migration of fibroblasts preferentially along the short axis of the wounds, making infiltration more efficient; or the guidance cues could have increased the migration speed of fibroblasts along the short axis of the wounds. We measured apparent orientations and apparent speeds of migrating fibroblasts and found a strong guidance effect of short axis restraint relative to long axis restraint, but no greater speed of fibroblast migration along the short axis of the wounds. This confirmed that mechanical environment, either directly or indirectly via structural cues, regulated the directionality of fibroblast migration during wound healing in our experimental model.

\section{Mechanical, Structural, and Chemical Guidance Cues in the Fibrin Wound Model}

Studies have shown that fibroblasts orient along the axis of greatest strain when exposed to anisotropic strains (Katsumi et al., 2002; Henshaw et al., 2006; Lee et al., 2008; Pang et al., 2011). Strain anisotropy measured in the fibrin wounds suggested that a mechanical guidance cue oriented along the short axis of the wounds was present in gels subjected to short axis restraint, whereas no mechanical guidance cue was present in gels subjected to long axis restraint. 
Studies have shown that fibroblasts orient along aligned structures such as fibers and microridges (Dickinson et al., 1994; Loesberg et al., 2007). Fiber alignment measured in the fibrin wounds suggested that a structural guidance cue oriented along the axis of mechanical restraint was present in gels subjected to long axis restraint and in gels subjected to short axis restraint. Fiber alignment was stronger for long axis restraint, suggesting any migration bias due to fiber alignment should be stronger in this group.

Multiple factors have been shown to attract fibroblasts into fibrin wound matrices. Fibrin and fibronectin (covalently bound to fibrin during crosslinking) are haptotactic agents (Naito et al., 1989, 1998; Thibault et al., 2007), and fibrin has binding sites for chemotactic agents, such as bFGF and PDGF (Mosesson et al., 2001). Also, dense populations of fibroblasts may selfgenerate chemotactic gradients by internalizing and degrading growth factors (Haugh, 2006; Scherber et al., 2012). We measured migration tracks of individual fibroblasts and discovered a unidirectional bias of fibroblast migration toward the center of the fibrin wounds for both long and short axis restraint. This suggested that a chemical guidance cue, such as a chemo- or haptotactic gradient, oriented toward the center of the wound was present in gels.

\section{Inference of Guidance Cues Biasing Fibroblast Migration}

For long axis restraint, we considered the possibility of a structural guidance cue oriented along the long axis of the fibrin wound acting simultaneously with a chemical guidance cue oriented toward the center of the fibrin wound. We used the computational model to infer whether this combination of guidance cues was consistent with the observed migration behavior. According 
to the model, the structural guidance cue had a negligible influence on the observed migration behavior. For short axis restraint, we considered the possibility of a mechanical guidance cue oriented along the short axis of the fibrin wound acting simultaneously with a chemical guidance cue oriented toward the center of the fibrin wound. According to the model, the mechanical guidance cue had a strong influence on the observed migration behavior. Overall, the modeling results were consistent with our measurements of mechanical anisotropy, which suggested the presence of a mechanical guidance cue only for short axis restraint, and inconsistent with our measurements of structural anisotropy, which suggested the presence of a structural guidance cue for both long and short axis restraint, where long axis restraint had the stronger cue.

The finding that fibroblasts in the fibrin wounds were sensitive to mechanical but not structural guidance cues was surprising because aligned fiber matrices have been shown to bias fibroblast migration (Dickinson et al., 1994; Provenzano et al., 2008; Liu et al., 2009). Either the fiber alignment was too weak to elicit a response, or other features of the environment rendered the fibroblasts insensitive to fiber alignment. Other studies have shown that sensitivity of cells to structural guidance cues is context dependent. Rajnicek et al. (2007) showed that a direct current electric field applied over a nano-grooved substrate caused cells to migrate perpendicular to the grooves. Similarly, Prodanov et al. (2010) showed that cyclic uniaxial strain applied to a nanogrooved substrate caused cells to orient perpendicular to the grooves. 


\section{Computational Model of Fibroblast Migration Guided by Environmental Cues}

The computational model produced migration behavior that was very similar to the migration behavior that we observed. The model required specification of only a displacement magnitude, displacement frequency, and displacement orientation probability distribution. In Chapter 4, we found that this model could reproduce random migration coefficients, a coarse descriptor of cell migration, for fibroblast migration biased by a single guidance cue (aligned collagen fibers). In this chapter, we found that this model can reproduce many detailed features of fibroblast migration and handle the biases of multiple simultaneous guidance cues. The model should be tested on additional data sets to determine the extent of its applicability. It is a potentially useful tool for predicting and interpreting migration behavior in response to guidance cues.

\section{Limitations}

We inferred the presence of a chemical guidance cue from the unidirectional bias of the fibroblast migration tracks toward the center of the fibrin wounds. Although we discussed evidence suggesting that we should expect chemo- or haptotactic gradients in the fibrin wounds, we cannot rule out other possible sources of the unidirectional bias. One alternative explanation might be that steric hindrance between cells enforced preferential migration of fibroblasts from the densely populated collagen gels into the cell-free fibrin wounds. Regardless, we do not need to know the source of the unidirectional bias in order to interpret the effect the mechanical guidance cue had on fibroblast migration in this study. 


\subsection{CONCLUSION}

Mechanical environment was an important regulator of fibroblast migration in our experimental wound healing model. Anisotropic remodeling strains were correlated with a directional bias of fibroblast migration that made infiltration of fibroblasts into the fibrin wounds more efficient. This bias was consistent with a direct effect of strain anisotropy on fibroblast migration and inconsistent with an effect of strain-induced fiber alignment. The mechanical guidance cue (strain anisotropy) did not increase the speed of fibroblast migration into the fibrin wounds. The migration behavior could be modeled as a biased random walk, where the bias due to environmental guidance cues was accounted for in the shape of a displacement orientation probability distribution. 


\subsection{FIGURES AND TABLES}

A. Long Axis Restraint

B. Short Axis Restraint

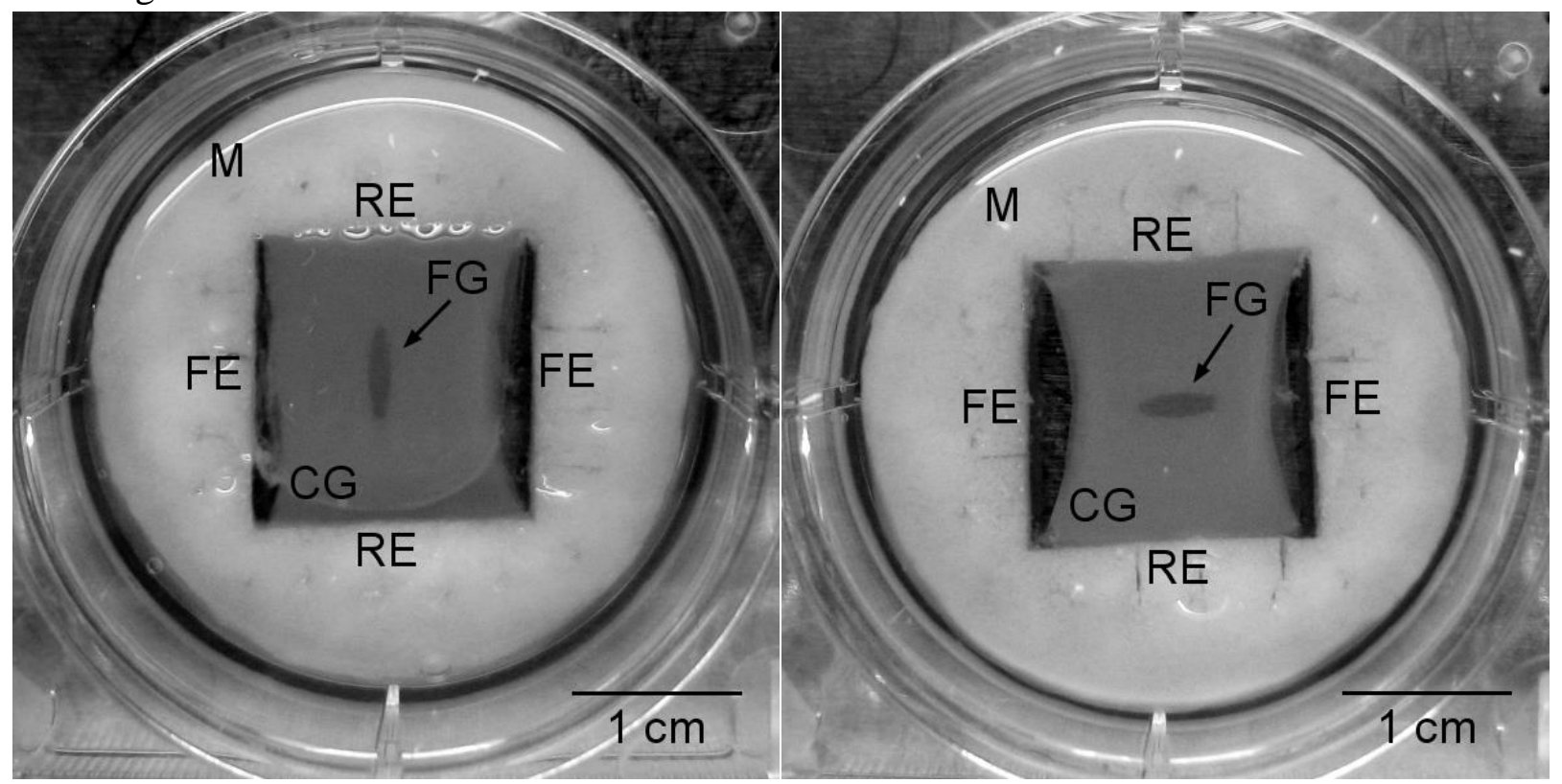

Figure 5.1. Example images of the three dimensional collagen-fibrin model wounds. Fibroblast-populated collagen gels (CG) were cast in square polyethylene molds (M). After one day of culture, a slit was cut in the collagen gel and the gap was filled with a fibrin gel (FG). After the fibrin gel polymerized, two opposing sides of the collagen gel were cut free from the polyethylene mold $(\mathrm{FE}=$ free edge, $\mathrm{RE}=$ restrained edge $)$ to create a uniaxial mechanical restraint acting either along the long axis of the elliptical fibrin wound (A) or along the short axis of the wound (B). Fibroblasts migrated into the fibrin wounds during an additional two days of culture. The images shown were taken one day after creating the fibrin wounds. The intensity histograms of these images were adjusted to improve contrast in grayscale. 
A

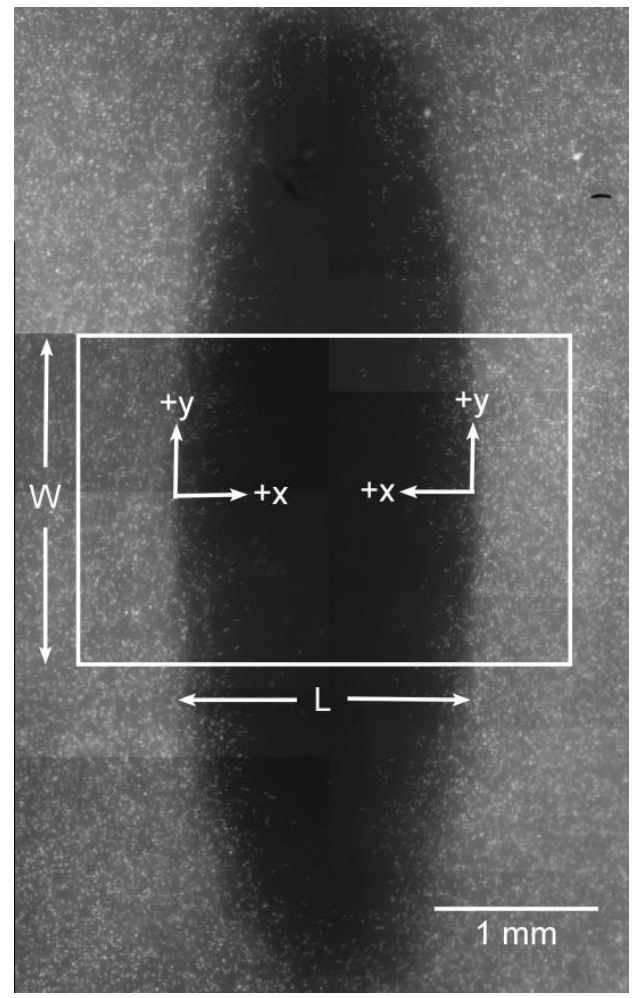

B

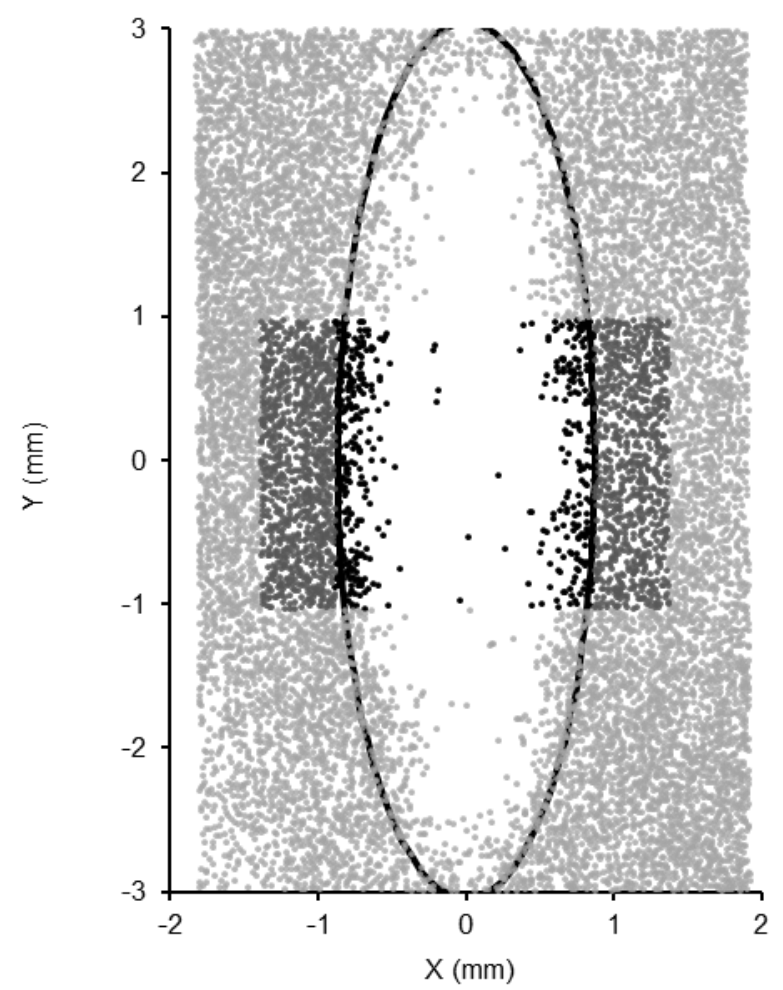

Figure 5.2. Example epi-fluorescence image used to acquire coordinates of cells and coordinates of the wound border. Cell nuclei were stained with DAPI. Overlapping fields were imaged on an epi-fluorescence microscope and registered to create a complete image of the fibrin wounds and surrounding collagen gel (A). Because collagen exhibited stronger autofluorescence than fibrin, the gradient of background fluorescence at the collagen-fibrin interface indicated the location of the wound border. Border coordinates were fit to an ellipse (black curve in B). Cell coordinates were identified as fluorescence intensity peaks after background subtraction and noise filtering (filled circles in B). We quantified migration of fibroblasts across a gap of length $\mathrm{L}$ and width $\mathrm{W}$, where $\mathrm{L}$ spanned the short axis of the wound and $\mathrm{W}$ spanned the middle third of the long axis of the wound (white box in A, dark circles in $\mathbf{B}$ ). On each side of the wound, a coordinate system was defined with centroid located at the wound edge and $+\mathrm{x}-$ direction toward the center of the wound (A). 
A. Long Axis Restraint

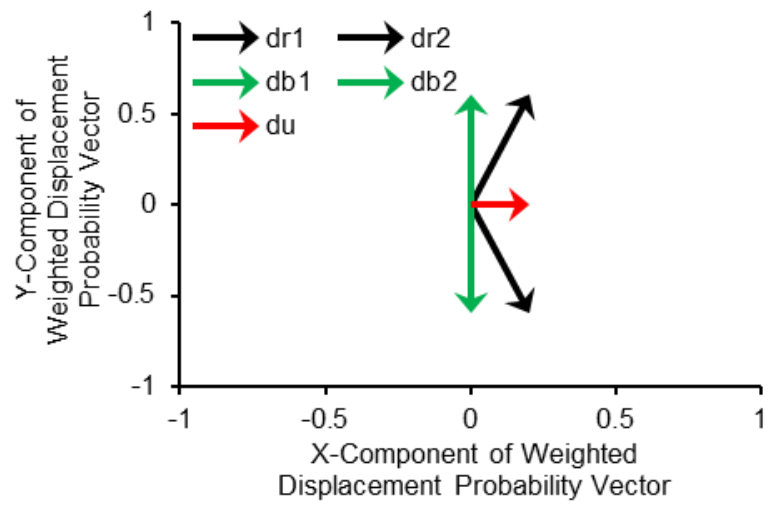

C. Long Axis Restraint

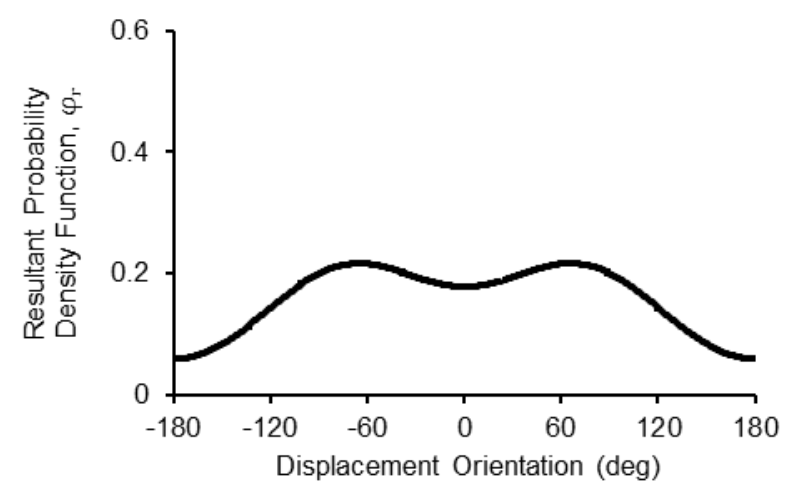

E

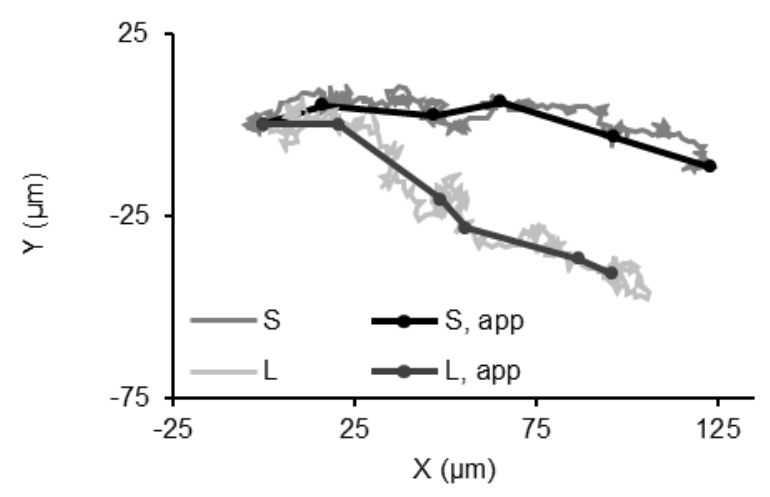

B. Short Axis Restraint

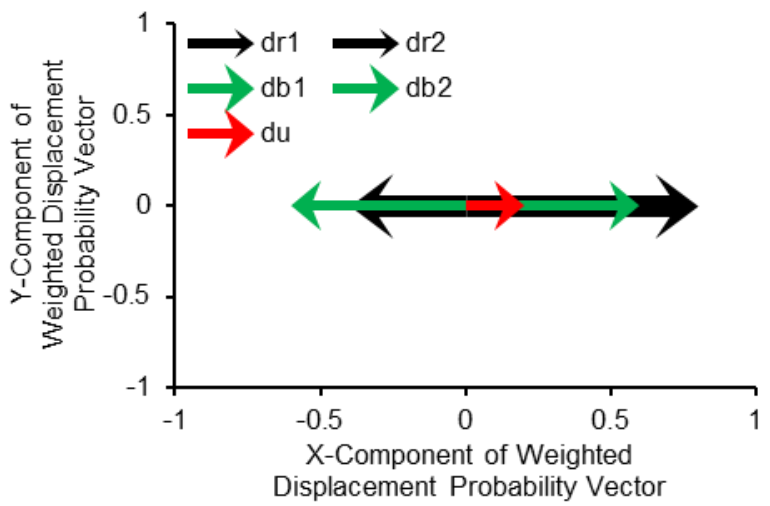

D. Short Axis Restraint

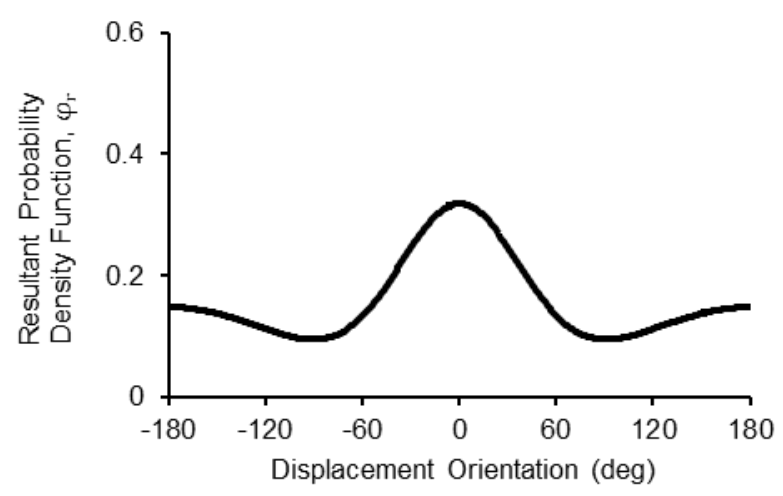

Figure 5.3. Computational model of fibroblast migration guided by environmental cues. We considered the possible influence of two guidance cues: a bidirectional cue, due to mechanical or structural anisotropy, oriented along the axis of mechanical restraint $\left(\bar{\theta}_{b}=90^{\circ}\right.$ for long axis restraint and $0^{\circ}$ for short axis restraint); and a unidirectional cue, due to chemo- or haptotaxis, oriented toward the center of the wound $\left(\bar{\theta}_{u}=0^{\circ}\right)$. We computed resultant displacement probability vectors $\left(\vec{d}_{r}\right)$ as a weighted sum of the displacement probability vectors representing the individual cues $\left(\vec{d}_{b}\right.$ and $\vec{d}_{u}$ ) (A and $\mathbf{B}$ ), calculated the associated resultant displacement orientation distribution $\left(\varphi_{r}\right)(\mathbf{C}$ and $\mathbf{D})$, and simulated migration tracks by randomly sampling the distribution to determine the orientation of each incremental cell displacement $(\mathbf{E})$. 
A

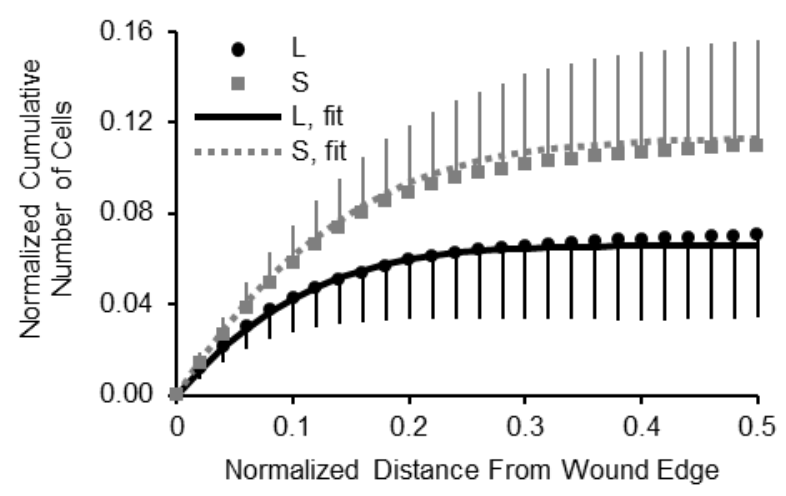

B

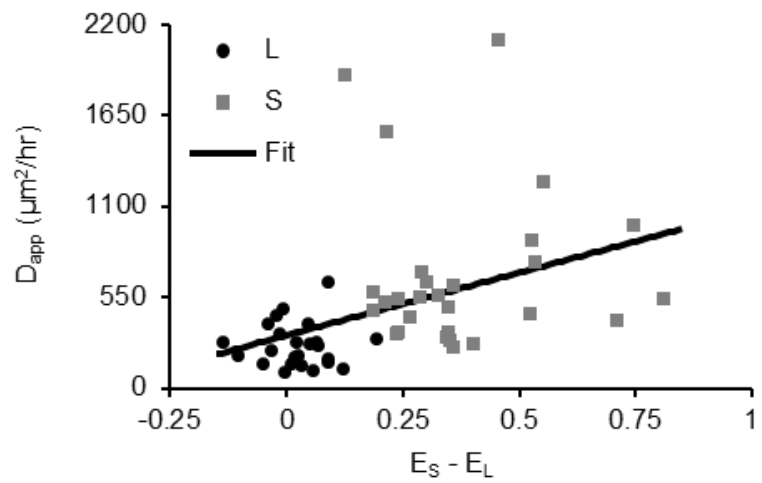

Figure 5.4. Cumulative distributions of fibroblasts and apparent diffusion coefficients. A. After allowing fibroblasts to migrate into the fibrin wounds over the course of two days, we calculated the cumulative number of cells that entered from the sides of the wounds as a function of distance from the edge of the wound to the center of the wound. The cumulative number of cells was normalized by the concentration of cells in the collagen gel multiplied by the area of the observation window. Distance was normalized by the short axis length of the wound. More fibroblasts travelled farther into the fibrin wounds with short axis restraint $(\mathrm{S})$ than with long axis restraint (L). In order to quantify the rate of infiltration of fibroblasts into the wounds, we calculated apparent diffusion coefficients by fitting the cumulative distributions to the diffusion equation (L, fit and $\mathrm{S}$, fit). B. The apparent diffusion coefficients derived from the fits $\left(\mathrm{D}_{\text {app }}\right)$ were positively correlated with strain anisotropy $\left(E_{S}-E_{L}\right)$. Least squares linear regression yielded a slope of $m=760 \mu \mathrm{m}^{2} / \mathrm{hr}$ with a standard error of $S . E \cdot m=240 \mu \mathrm{m}^{2} / \mathrm{hr}(\mathrm{p}<0.05$ by two-tailed test of the t-statistic $t=m / S . E_{\cdot m}$ ) and correlation coefficient of $r^{2}=0.17 . \mathrm{N}=28$ for short axis restraint and 24 for long axis restraint. 
A

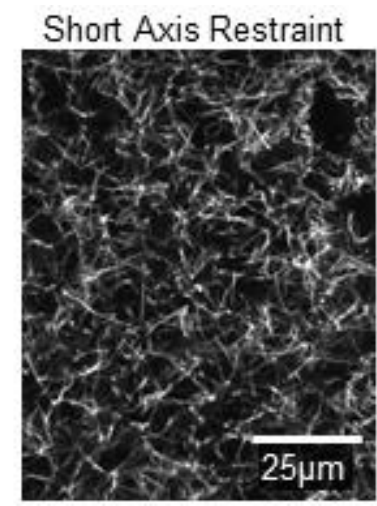

B
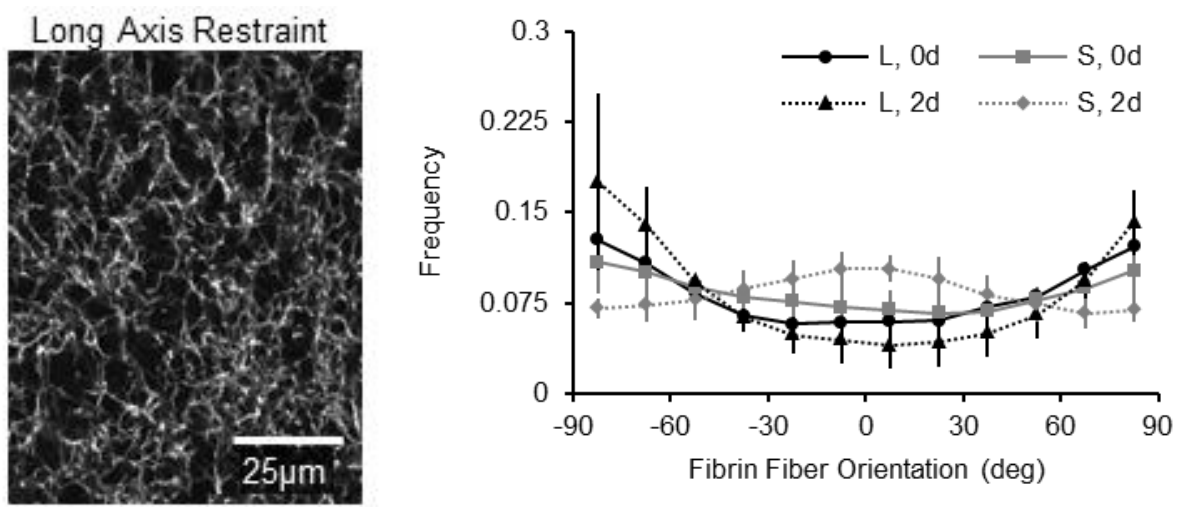

$\mathrm{C}$

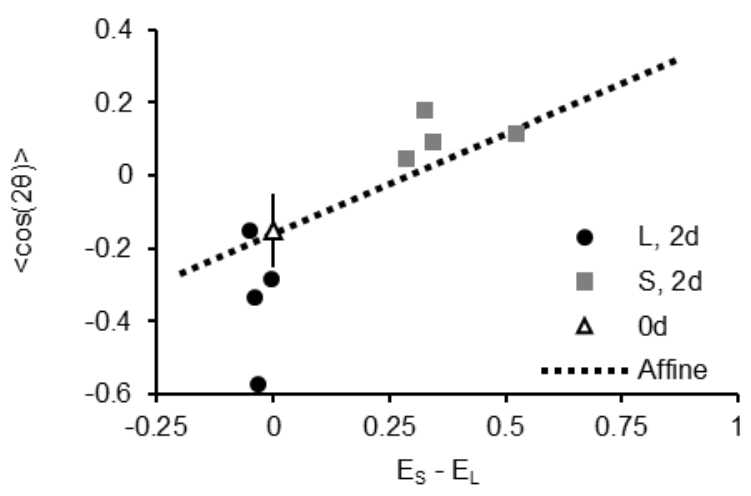

Figure 5.5. Fibrin matrix structure measured zero and two days after wounding. A. We prepared gels for histology, stained sections with eosin, and acquired images of fibrin fibers on a confocal microscope (Day 2 shown). Three fields of fibrin fibers per gel were sampled in three gels per group at Day 0 and four gels per group at Day 2. B. We used an intensity gradient detection algorithm, Fiber3 (Karlon et. al., 1999), to compute sets of fiber orientations for each sample and then averaged histograms of fiber orientations across the samples for each group. Immediately after creating the wounds, the long axis restraint group (L, Od) and short axis restraint group $(\mathrm{S}, 0 \mathrm{~d})$ had similar structures, showing a slight alignment of fibers along the long axis ( \pm 90 degrees). Two days after creating the wounds, the fibers of the long axis restraint group (L, 2d) became slightly more aligned along the long axis, while the fibers of the short axis restraint group $(\mathrm{S}, 2 \mathrm{~d})$ became slightly aligned along the short axis (0 degrees). C. Structural anisotropy $(<\cos (2 \theta)>)$ was positively correlated with strain anisotropy $\left(E_{S}-E_{L}\right)$, and the measurements agreed reasonably with the values predicted assuming affine deformation of the average initial structure (Affine). 
A. Experiment - Long Axis Restraint

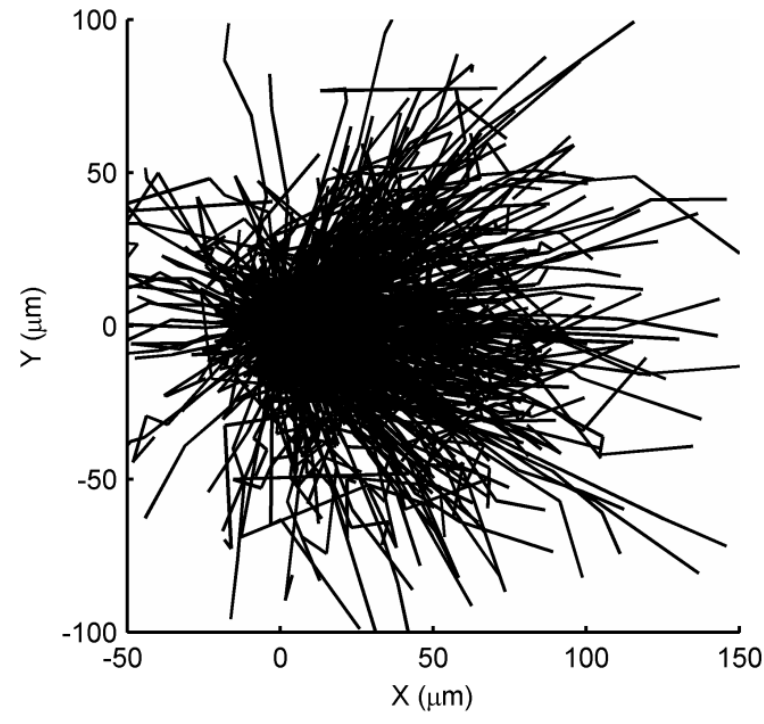

C. Model - Long Axis Restraint

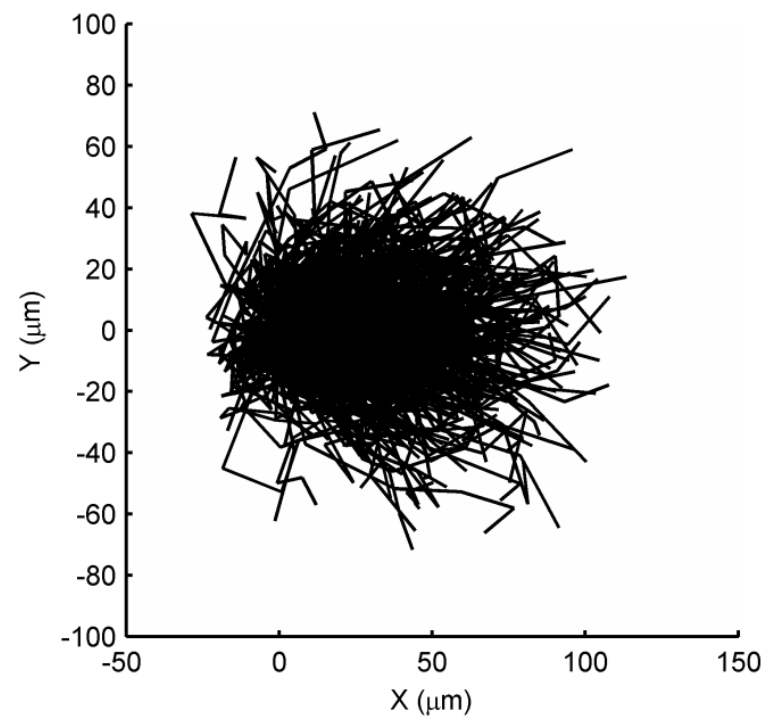

B. Experiment - Short Axis Restraint

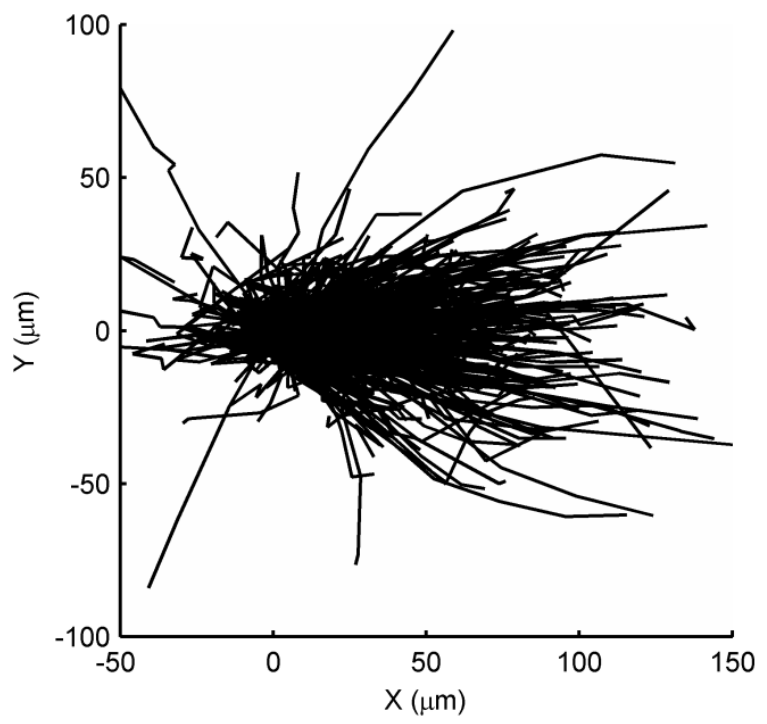

D. Model - Short Axis Restraint

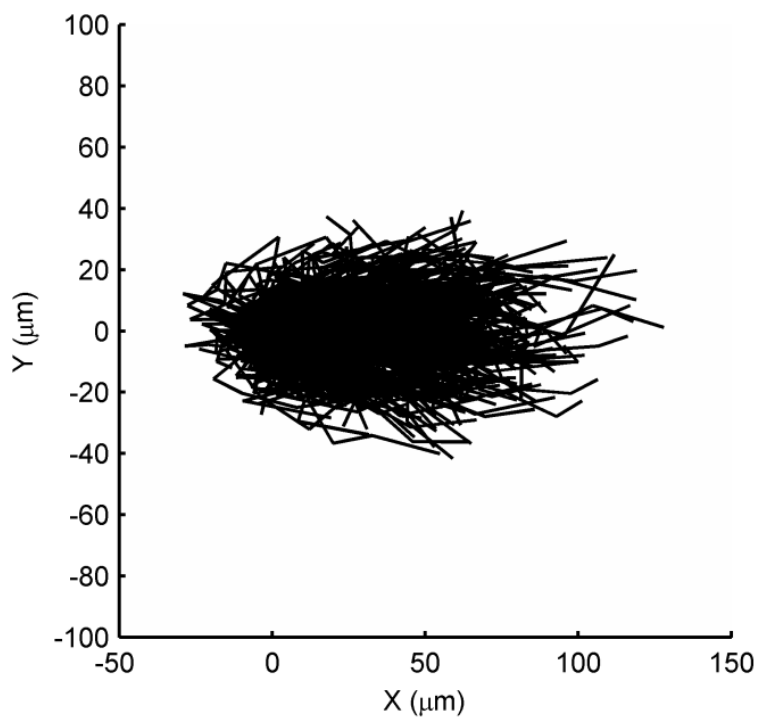

Figure 5.6. Fibroblast migration tracks measured after one day of wound infiltration. Every hour for a period of six hours, we captured positions of fibroblasts. Migration tracks were measured in three gels per group, yielding a total of 568 cells tracked for long axis restraint and 471 cells tracked for short axis restraint. The computational model simulated $\sim 2 \mu \mathrm{m}$ displacements of fibroblasts every minute and saved positions every hour. A random sample taken from a probability distribution determined the orientation of each displacement. A. For long axis restraint, fibroblasts migrated preferentially toward the center of the wound $(+x-$ direction). B. For short axis restraint, fibroblasts also migrated preferentially toward the center of the wound and migration tracks were aligned more strongly along the short axis (x-axis). C and D. The computational model of fibroblast migration reproduced the bulk of the fibroblast migration tracks for both mechanical conditions, except for the subset of cells with relatively high net displacement. 
A. Experiment - Long Axis Restraint

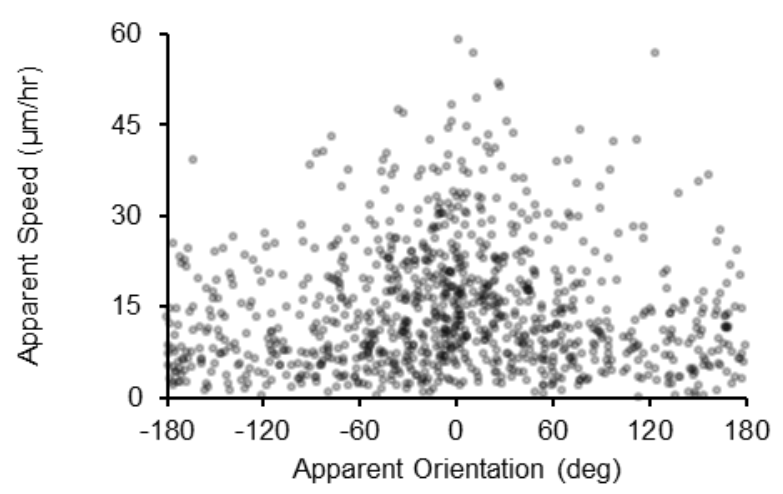

C. Model - Long Axis Restraint

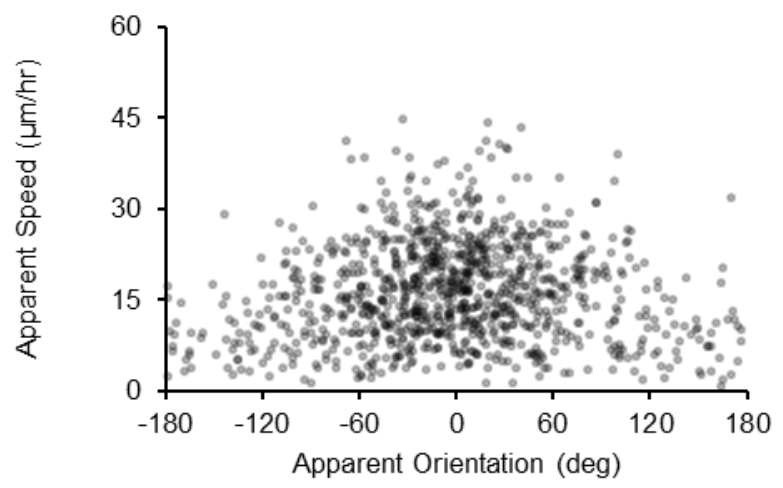

B. Experiment - Short Axis Restraint

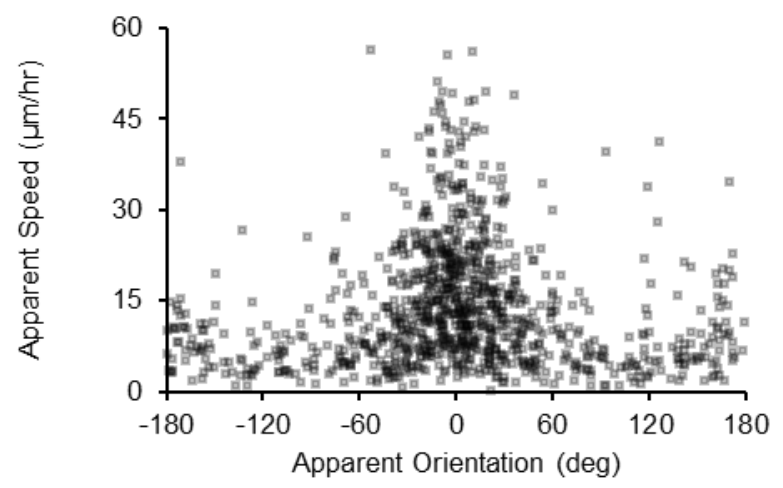

D. Model - Short Axis Restraint

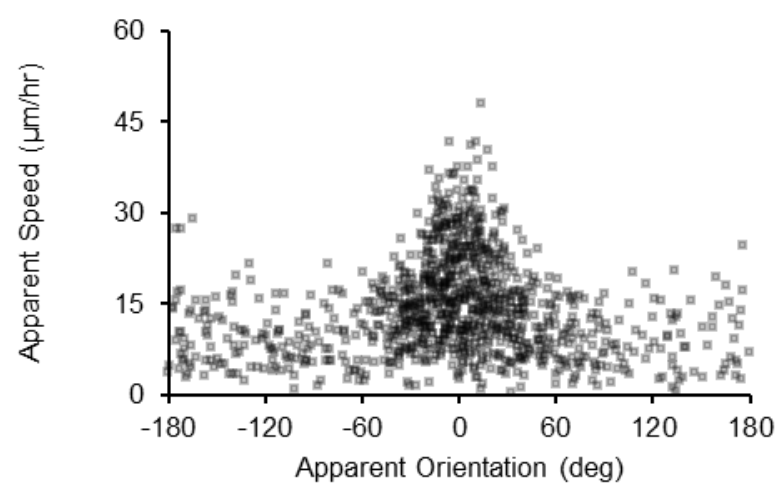

Figure 5.7. Apparent speeds and apparent orientations of fibroblasts. For each one hour time interval, apparent speeds and apparent orientations were calculated from the displacement vectors of the cells. In these plots, 0 degrees corresponds to the direction along the short axis of the wound from edge to center (+x-direction). A. For long axis restraint, the data were clustered around 0 degrees, indicating motion was biased toward the center of the wound. Apparent speeds were also higher around 0 degrees. B. Compared to long axis restraint, short axis restraint showed stronger clustering of data around 0 degrees, lower apparent speeds around 90 and -90 degrees, and a secondary cluster of data around $+/-180$ degrees. C and D. The computational model of fibroblast migration reproduced all of the observed features of the experimental data, except for the subset of displacements with relatively high apparent speeds seen in the experimental data. 
A

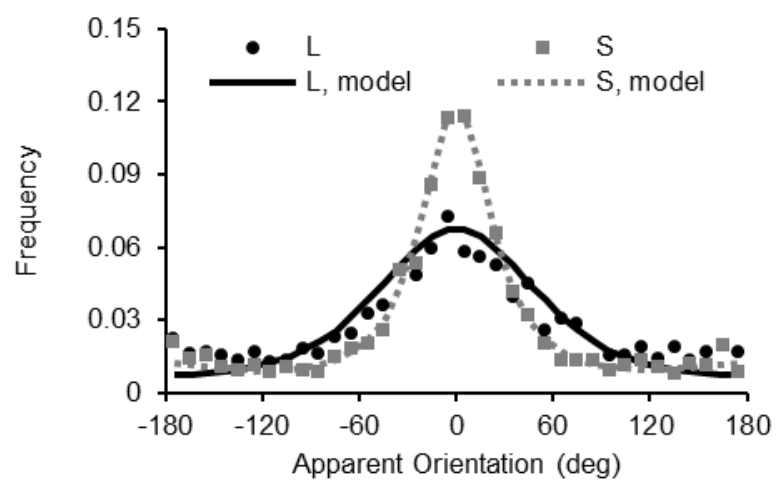

C. Long Axis Restraint

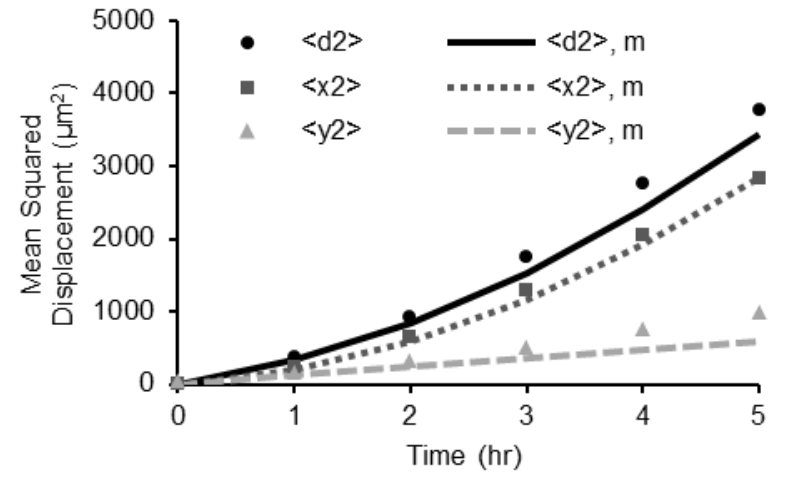

E. Long Axis Restraint

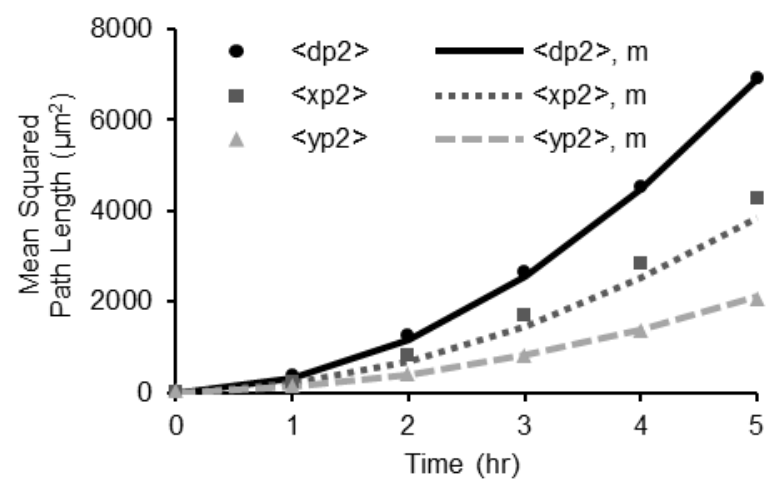

B

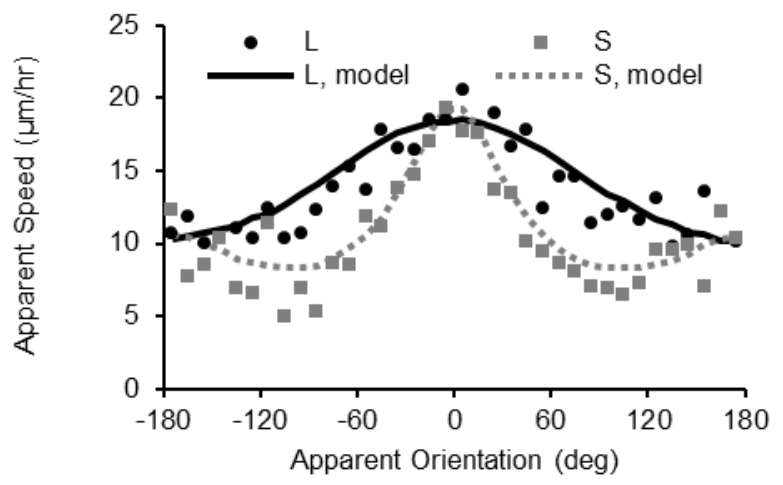

D. Short Axis Restraint

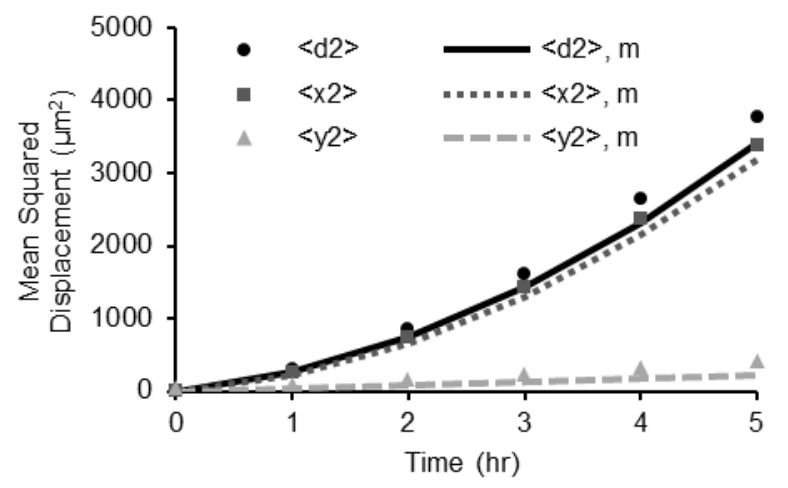

F. Short Axis Restraint

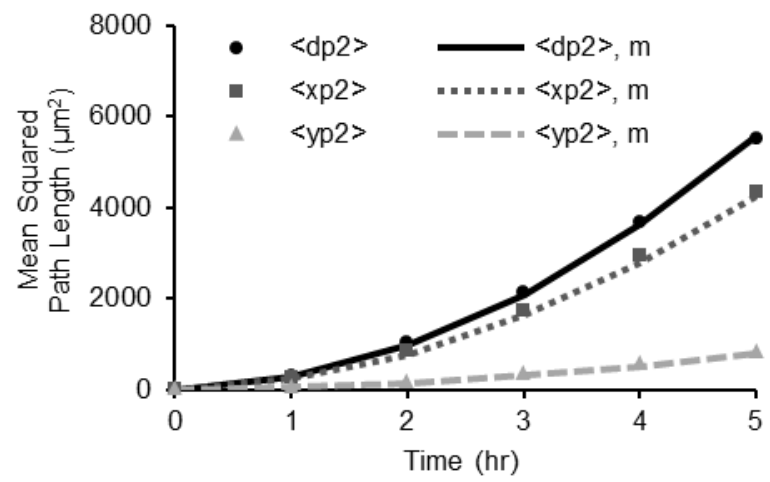

Figure 5.8. Quantitative summary of migration data and comparison to computational model. Markers indicate experimental data and curves indicate model data. A. Fibroblast displacements were oriented more strongly along the short axis ( 0 and +/-180 degrees) with short axis restraint (S) than with long axis restraint (L). B. Apparent speeds along the short axis were similar for short and long axis restraint, but apparent speeds along the long axis (-90 and 90 degrees) were lower for short axis restraint than for long axis restraint. C., D., E., and F. The mean squared displacement curves were nonlinear but not as steep as the mean squared path curves, indicating that migration lay between straight line motion and a random walk. The short axis (x) bias was stronger with short axis restraint than with long axis restraint. The computational model of fibroblast migration reproduced all of the experimental data shown in these figures. 
A

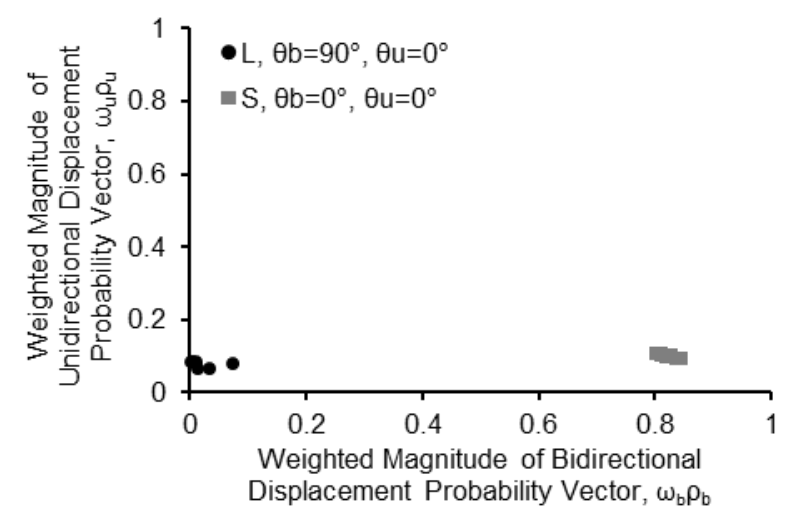

B

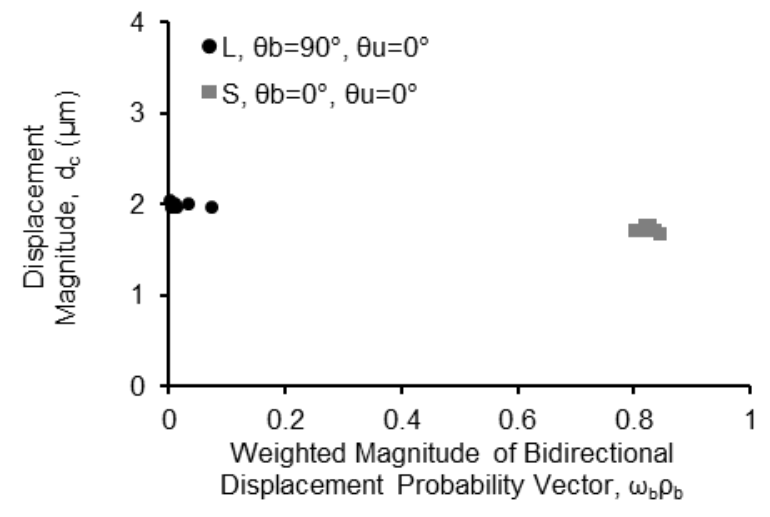

C. Long Axis Restraint

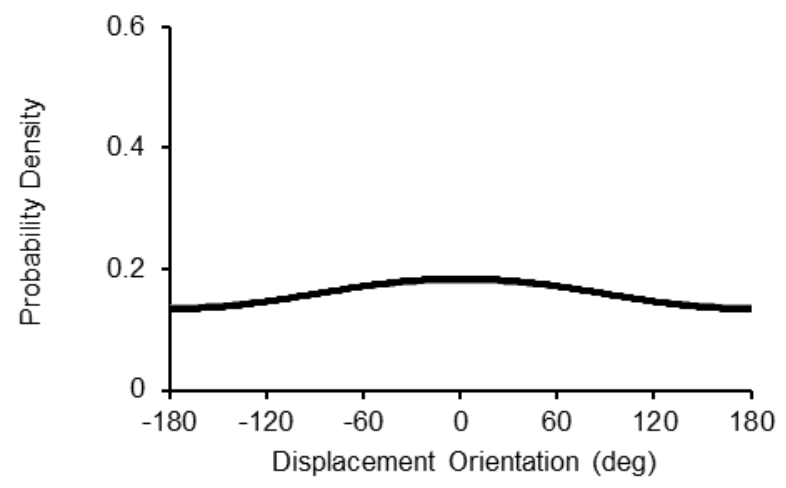

D. Short Axis Restraint

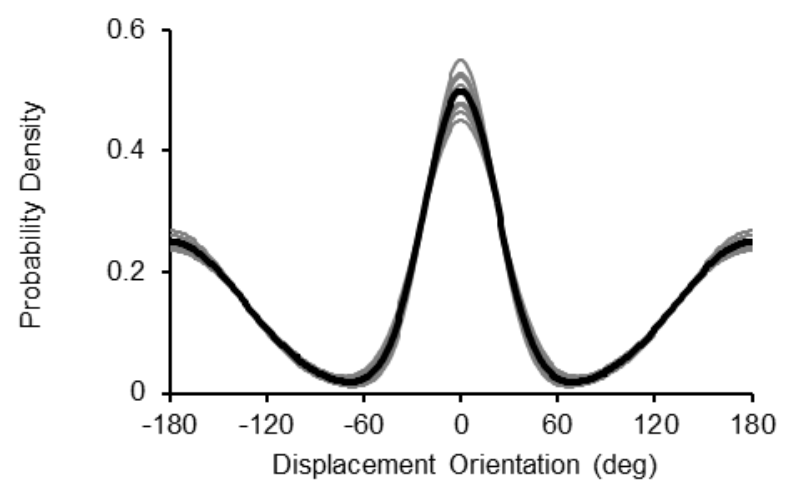

Figure 5.9. Computational model parameter optimization. Three free model parameters - the displacement magnitude $\left(d_{c}\right)$, the weighted magnitude of the bidirectional displacement probability vector $\left(\omega_{b} \rho_{b}\right)$, and the weighted magnitude of the unidirectional displacement probability vector $\left(\omega_{u} \rho_{u}\right)$ - were determined by fitting model results to experimental data. A multi-parameter, multi-objective optimization problem was solved by minimizing a weighted sum of two objectives: 1) the normalized root mean squared difference between model and experiment of the apparent orientation histogram and 2) the normalized root mean squared difference between model and experiment of the average apparent speed versus apparent orientation curve. Weight factors were varied from 0.01 to 0.99 in order to obtain sets of solutions representing goodness-of-fit compromises between the data sets. A. For long axis restraint $(\mathrm{L})$, the best fits were obtained with a small unidirectional bias $\left(\omega_{u} \rho_{u}\right)$ toward the center of the wound and negligible bidirectional bias $\left(\omega_{b} \rho_{b}\right)$ along the long axis of the wound. For short axis restraint $(S)$, the best fits were obtained with a small unidirectional bias toward the center of the wound and a very strong bidirectional bias along the short axis of the wound. B. In the $d_{c}$ versus $\omega_{b} \rho_{b}$ plane, the model parameter phase space showed clustering of solutions around a displacement magnitude of $\sim 2 \mu \mathrm{m}$. C and D. Resultant probability density functions for displacement orientations. The gray curves show the range of shapes given by all solutions, with the mean shown as the thick black curve. 
Table 5.1. Geometry, mechanics, and structure of fibrin wounds; fibroblast concentration and infiltration rate.

\begin{tabular}{|c|c|c|c|c|}
\hline & & & Long Axis Restraint & $\underline{\text { Short Axis Restraint }}$ \\
\hline Short Axis Length & $\mathrm{L}_{\mathrm{S}}$ & $\mathrm{mm}$ & $1.1 \pm 0.3$ & $1.5 \pm 0.3$ \\
\hline Long Axis Length & $\mathrm{L}_{\mathrm{L}}$ & $\mathrm{mm}$ & $4.9 \pm 0.5$ & $3.8 \pm 0.7$ \\
\hline Short Axis Remodeling Strain & $\mathrm{E}_{\mathrm{S}}$ & & $-0.074 \pm 0.078$ & $0.085 \pm 0.116$ \\
\hline Long Axis Remodeling Strain & $\mathrm{E}_{\mathrm{L}}$ & & $-0.098 \pm 0.056$ & $-0.290 \pm 0.087$ \\
\hline Mechanical Anisotropy & $\Delta \mathrm{E}=\mathrm{E}_{\mathrm{S}}-\mathrm{E}_{\mathrm{L}}$ & & $0.025 \pm 0.072 \sim$ & $0.376 \pm 0.175^{\#}$ \\
\hline Fibrin Mean Angle & MA & $\operatorname{deg}$ & $-83 \pm 2$ & $0 \pm 22$ \\
\hline Fibrin Mean Vector Length & MVL & & $0.35 \pm 0.19$ & $0.14 \pm 0.06$ \\
\hline Structural Anisotropy & $<\cos (2 \theta)>$ & & $-0.34 \pm 0.18^{\#}$ & $0.11 \pm 0.06^{\#}$ \\
\hline Remote Cell Concentration & $\mathrm{C}_{\mathrm{R}}$ & $\mathrm{mm}^{-2}$ & $610 \pm 210$ & $570 \pm 260$ \\
\hline Apparent Diffusion Coefficient & $\mathrm{D}_{\mathrm{app}}$ & $\mu \mathrm{m}^{2} / \mathrm{hr}$ & $260 \pm 130 *$ & $680 \pm 480 *$ \\
\hline
\end{tabular}

${ }^{*} \mathrm{p}<0.05$ by unpaired, two-tailed, student's t-test for Long Axis Restraint vs. Short Axis Restraint

$\# \mathrm{p}<0.05$ by one-sample, two-tailed, student's t-test against hypothetical value of 0

$\sim p=$ n.s. by one-sample, two-tailed, student's t-test against hypothetical value of 0 
Table 5.2. Migration Model Parameters.

\begin{tabular}{|c|c|c|c|c|}
\hline & & & Long Axis Restraint & $\underline{\text { Short Axis Restraint }}$ \\
\hline $\begin{array}{l}\text { Weighted Magnitude of Bidirectional } \\
\text { Displacement Probability Vector }\end{array}$ & $\omega_{b} \rho_{b}$ & & $0.021 \pm 0.026$ & $0.824 \pm 0.015$ \\
\hline $\begin{array}{c}\text { Angle of Bidirectional } \\
\text { Displacement Probability Vector }\end{array}$ & $\bar{\theta}_{b}$ & deg & 90 & 0 \\
\hline $\begin{array}{l}\text { Weighted Magnitude of Unidirectional } \\
\text { Displacement Probability Vector }\end{array}$ & $\omega_{u} \rho_{u}$ & & $0.075 \pm 0.008$ & $0.098 \pm 0.005$ \\
\hline $\begin{array}{c}\text { Angle of Unidirectional } \\
\text { Displacement Probability Vector }\end{array}$ & $\bar{\theta}_{u}$ & deg & 0 & 0 \\
\hline Displacement Magnitude & $d_{c}$ & $\mu \mathrm{m}$ & $2.0 \pm 0.0$ & $1.7 \pm 0.0$ \\
\hline Displacement Frequency & $f$ & $\min ^{-1}$ & 1 & 1 \\
\hline
\end{tabular}




\subsection{REFERENCES}

Atance J, Yost MJ \& Carver W (2004). Influence of the extracellular matrix on the regulation of cardiac fibroblast behavior by mechanical stretch. Journal of Cellular Physiology 200, 377386.

Berry CC, Shelton JC, Bader DL \& Lee DA (2003). Influence of external uniaxial cyclic strain on oriented fibroblast-seeded collagen gels. Tissue Engineering 9, 613-624.

Diaz-Araya G, Borg TK, Lavandero S, Loftis MJ \& Carver W (2003). IGF-1 modulation of rat cardiac fibroblast behavior and gene expression is age-dependent. Cell Communication and Adhesion 10, 155-165.

Dickinson RB, Guido S \& Tranquillo RT (1994). Biased cell migration of fibroblasts exhibiting contact guidance in oriented collagen gels. Annals of Biomedical Engineering 22, 342-356.

Grassl ED, Oegema TR \& Tranquillo RT (2003). A fibrin-based arterial media equivalent. Journal of Biomedical Materials Research Part A 66, 550-561.

Grinnell F, Rocha LB, Iucu C, Rhee S \& Jiang H (2006). Nested collagen matrices: a new model to study migration of human fibroblast populations in three dimensions. Experimental Cell Research 312, 86-94.

Haugh JM (2006). Deterministic model of dermal wound invasion incorporating receptormediated signal transduction and spatial gradient sensing. Biophysical Journal 90, 22972308.

Henshaw DR, Attia E, Bhargava M \& Hannafin JA (2006). Canine ACL fibroblast integrin expression and cell alignment in response to cyclic tensile strain in three-dimensional collagen gels. Journal of Orthopaedic Research 24, 481-490.

Karlon WJ, Covell JW, McCulloch AD, Hunter JJ \& Omens JH (1998). Automated measurement of myofiber disarray in transgenic mice with ventricular expression of ras. The Anatomical Record 252, 612-625.

Karlon WJ, Hsu PP, Li S, Chien S, McCulloch AD \& Omens JH (1999). Measurement of orientation and distribution of cellular alignment and cytoskeletal organization. Annals of Biomedical Engineering 27, 712-720.

Katsumi A, Milanini J, Kiosses WB, Del Pozo MA, Kaunas R, Chien S, Hahn KM \& Schwartz MA (2002). Effects of cell tension on the small GTPase Rac. The Journal of Cell Biology 158, 153-164.

Kolodney MS \& Wysolmerski RB (1992). Isometric contraction by fibroblasts and endothelial cells in tissue culture: a quantitative study. The Journal of Cell Biology 117, 73-82. 
Lee EJ, Holmes JW \& Costa KD (2008). Remodeling of engineered tissue anisotropy in response to altered loading conditions. Annals of Biomedical Engineering 36, 1322-1334.

Liu Y, Ji Y, Ghosh K, Clark RAF, Huang L \& Rafailovich MH (2009). Effects of fiber orientation and diameter on the behavior of human dermal fibroblasts on electrospun PMMA scaffolds. Journal of Biomedical Materials Research Part A 90, 1092-1106.

Loesberg WA, Te Riet J, Van Delft FCMJM, Schön P, Figdor CG, Speller S, Van Loon JJWA, Walboomers XF \& Jansen JA (2007). The threshold at which substrate nanogroove dimensions may influence fibroblast alignment and adhesion. Biomaterials 28, 3944-3951.

Mardia K V \& Jupp PE (2000). Directional Statistics. Chichester, UK.

Mosesson MW, Siebenlist KR \& Meh DA (2001). The structure and biological features of fibrinogen and fibrin. Annals of the New York Academy of Sciences 936, 11-30.

Naito M, Hayashi T, Kuzuya M, Funaki C, Asai K \& Kuzuya F (1989). Fibrinogen is chemotactic for vascular smooth muscle cells. FEBS Letters 247, 358-360.

Naito M, Nomura H, Iguchi A, Thompson WD \& Smith EB (1998). Effect of crosslinking by factor XIIIa on the migration of vascular smooth muscle cells into fibrin gels. Thrombosis Research 90, 111-116.

Pang Y, Wang X, Lee D \& Greisler HP (2011). Dynamic quantitative visualization of single cell alignment and migration and matrix remodeling in 3-D collagen hydrogels under mechanical force. Biomaterials 32, 3776-3783.

Prodanov L, Te Riet J, Lamers E, Domanski M, Luttge R, Van Loon JJWA, Jansen JA \& Walboomers XF (2010). The interaction between nanoscale surface features and mechanical loading and its effect on osteoblast-like cells behavior. Biomaterials 31, 7758-7765.

Provenzano PP, Inman DR, Eliceiri KW, Trier SM \& Keely PJ (2008). Contact guidance mediated three-dimensional cell migration is regulated by Rho/ROCK-dependent matrix reorganization. Biophysical Journal 95, 5374-5384.

Rajnicek AM, Foubister LE \& McCaig CD (2007). Prioritising guidance cues: directional migration induced by substratum contours and electrical gradients is controlled by a rho/cdc42 switch. Developmental Biology 312, 448-460.

Robinson PS, Johnson SL, Evans MC, Barocas VH \& Tranquillo RT (2008). Functional tissueengineered valves from cell-remodeled fibrin with commissural alignment of cell-produced collagen. Tissue Engineering: Part A 14, 83-95.

De Rossi A, Rocha LB \& Rossi MA (2007). Application of fluorescence microscopy on hematoxylin and eosin-stained sections of healthy and diseased teeth and supporting structures. Journal of Oral Pathology \& Medicine 36, 377-381. 
Scherber C, Aranyosi AJ, Kulemann B, Thayer SP, Toner M, Iliopoulos O \& Irimia D (2012). Epithelial cell guidance by self-generated EGF gradients. Integrative Biology 4, 259-269.

Shreiber DI, Enever PAJ \& Tranquillo RT (2001). Effects of pdgf-bb on rat dermal fibroblast behavior in mechanically stressed and unstressed collagen and fibrin gels. Experimental Cell Research 266, 155-166.

Thibault MM, Hoemann CD \& Buschmann MD (2007). Fibronectin, vitronectin, and collagen I induce chemotaxis and haptotaxis of human and rabbit mesenchymal stem cells in a standardized transmembrane assay. Stem Cells and Development 16, 489-502.

Thomopoulos S, Fomovsky GM \& Holmes JW (2005). The development of structural and mechanical anisotropy in fibroblast populated collagen gels. Journal of Biomechanical Engineering 127, 742-750.

Tyagi SC, Lewis K, Pikes D, Marcello A, Mujumdar VS, Smiley LM \& Moore CK (1998). Stretch-induced membrane type matrix metalloproteinase and tissue plasminogen activator in cardiac fibroblast cells. Journal of Cellular Physiology 176, 374-382.

Williams C, Johnson SL, Robinson PS \& Tranquillo RT (2006). Cell sourcing and culture conditions for fibrin-based valve constructs. Tissue Engineering 12, 1489-1502. 


\section{CHAPTER 6: Agent-Based Model with Integrated Finite Element Analysis for Investigation of the Mechanobiology of Healing Myocardial Infarcts}

\subsection{INTRODUCTION}

The work in this thesis is part of a broader effort to advance our understanding of the regulation of infarct scar structure and its impact on heart function. We focused specifically on the regulation of collagen fiber alignment by fibroblasts during healing after myocardial infarction. Some groups have focused on the regulation of collagen content: studying matricellular proteins (McCurdy et al., 2011), matrix metalloproteinases and tissue inhibitors of matrix metalloproteinases (Lindsey \& Zamilpa, 2012), signaling molecules such as angiotensin II and transforming growth factor-beta (Rosenkranz, 2004), and inflammatory cells that precede the arrival of fibroblasts at the site of infarction (Frangogiannis, 2008). Others have studied the relationship between infarct structure, mechanics, and heart function (Holmes et al., 1997, 2005; Mazhari et al., 2000; Fomovsky \& Holmes, 2010).

Computational models may be critical for ultimately linking diverse molecular and cellular mechanisms regulating infarct scar structure both horizontally—-for example, integrating into our agent-based model the influence of growth factors secreted by inflammatory cells on fibroblast migration, proliferation, collagen synthesis, and generation of traction forces (Dewald et al., 2005; Mitchell et al., 2007; Bujak \& Frangogiannis, 2007) — and vertically-for example, to predict how interventions that change the structure of infarct scar affect the mechanical properties of the scar, which in turn affect heart function (Rane \& Christman, 2011). Toward 
this end, finite element models of the heart are becoming quite advanced (Kroon et al., 2008; Arts et al., 2012) and efforts have recently been made to model how inflammatory cells interact with fibroblasts to regulate collagen content during infarct healing (Jin et al., 2011), in addition to our effort to quantitatively model environmental guidance cues and their influence on fibroblast and collagen fiber alignment (Rouillard \& Holmes, 2012). Integrating such models, however, remains a challenge.

Because of the important influence of mechanical guidance cues on collagen fiber remodeling during infarct healing (Fomovsky et al., 2012; Rouillard \& Holmes, 2012), integrating our agentbased model with a finite element model of the heart is an exciting and potentially important objective. Currently, our model assumes that strains are homogeneous throughout the infarct region and do not change over time. The assumption that the strains do not change over time may be reasonable under certain conditions and is supported by some experimental evidence (Fomovsky \& Holmes, 2010). However, this cannot be assumed to hold in general. The strains acting on an infarct are determined by its structural properties and by the loads acting through the heart wall, which are determined by the chamber pressure and geometry of the heart. Consequently, a feedback loop may exist during infarct healing, where the pattern of mechanical strain guides structural remodeling of the infarct, which then changes the infarct strains.

This feedback could cause the structural and mechanical properties of healing infarcts to evolve in ways we cannot capture with the current model. For example, our model does not currently capture the spatial heterogeneity of the collagen fiber structure of infarct scar. We have observed that infarct scar tissue is often composed of domains on the order of several hundred microns in 
size that are more highly aligned than the infarct as a whole, but which vary in mean orientation from domain to domain (Fig. 6.1). By imposing a static strain field in the model, we may restrict this heterogeneity from developing. Coupling a finite element model to our agent-based model would allow the model to predict the co-evolution of the structural and mechanical properties of healing infarcts.

We developed a finite element model of an infarct embedded in a sheet of myocardium, and we formulated an approach to coupling the finite element model to our agent-based model. Our approach was to replace the static field representation of mechanical strain in the agent-based model with strains computed by the finite element model. We used the agent-based model to predict structural remodeling over a period of time and then used the local fiber orientation histograms predicted by the agent-based model to calculate constitutive properties for each element in the finite element model. We used the finite element model to simulate loading of the infarct to end-diastole and end-systole, calculated systolic strains, and updated the strains in the agent-based model. Zahedmanesh \& Lally (2012) recently published a similar strategy to investigate the effect of fluid flow on intimal hyperplasia of vascular tissue engineering scaffolds.

We used the finite element model to investigate how infarct strains and mechanical guidance cues change in response to perturbations of infarct structure (collagen area fraction and fiber mean vector length). We also tested whether spatial heterogeneity of the collagen fiber structure of infarct scar can be explained as an alignment response of fibroblasts to spatially heterogeneous mechanical guidance cues. 


\subsection{METHODS}

\section{Agent-Based Model}

The agent-based model (ABM) was unchanged except for the mechanical strain field, which was imported from the solution of the finite element model.

\section{Finite Element Model}

Geometry, Boundary Conditions, and Mesh

The finite element model (FEM) represented a section of the anterior wall of the left ventricle cut parallel to the epicardial surface as a thin, square slab of material (Fig. 6.2a). In the FEM, the xaxis corresponded to the circumferential direction in the left ventricle, the y-axis corresponded to the longitudinal direction, and the z-axis corresponded to the transmural direction. Estimated circumferential and longitudinal wall stresses were applied to the side faces of the slab. Six nodal displacements at four corners of the slab were fixed in order to prevent rigid body translation and rotation (Fig. 6.2b). The size of the slab was chosen by first defining an inner square region with side length $L_{i}=500 \mu m$ matched to the size of the ABM, and then specifying an outer side length $L_{o}=2000 \mu \mathrm{m}$ such that the computed deformations in the center of the slab changed less than $5 \%$ when $L_{o}$ was doubled (Fig. 6.2c). The ABM domain contained a circular infarct region with radius $R=113 \mu \mathrm{m}$ matched to that of the ABM. Within the ABM 
domain, the slab was divided into a mesh of 50 by 50 square elements. Outside the ABM domain, the mesh was expanded to the edges of the slab using trapezoidal elements. The number of elements within the ABM domain $N_{e}=2500$ was chosen such that the computed deformations in the center of the slab changed less than $5 \%$ when $N_{e}$ was quadrupled (Fig. 6.2d).

\section{Wall Stress-Stretch Curves}

Wall stress-stretch curves for passive and active myocardium were estimated from the enddiastolic pressure-volume relationship (EDPVR) and end-systolic pressure-volume relationship (ESPVR) of control rat left ventricles (Fomovsky \& Holmes, 2010). Previously, our lab fit pressure-volume data from control hearts to an EDPVR of the form

$P_{D}=e^{k\left(V_{D}-V_{D}^{0}\right)}$

with $k=4.9 \ln \left(\mathrm{cm} \mathrm{H}_{2} \mathrm{O}\right) / \mathrm{ml}$ and $V_{D}^{0}=0.092 \mathrm{ml}$

and an ESPVR of the form

$P_{S}=E_{\max }\left(V_{S}-V_{S}^{0}\right)$

with $E_{\max }=675 \mathrm{~cm} \mathrm{H}_{2} \mathrm{O} / \mathrm{ml}$ and $V_{S}^{0}=0.042 \mathrm{ml}$. At baseline, the end-diastolic pressure was $P_{E D}=6.9 \mathrm{~cm} \mathrm{H}_{2} \mathrm{O}$ and the end-systolic pressure was $P_{E S}=115 \mathrm{~cm} \mathrm{H}_{2} \mathrm{O}$ (Fig. 6.3a).

We used $V_{D}^{0}$ as an estimate of the unloaded endocardial volume and computed wall stresses and wall stretches for volumes ranging from the unloaded volume $V_{D}^{0}=0.092 \mathrm{ml}$ to 1.5 times the baseline end-diastolic volume $1.5 V_{E D}=1.5(0.48 \mathrm{ml}) \sim 0.75 \mathrm{ml}$. As in the experimental study, we assumed the left ventricle had the shape of a prolate spheroid truncated halfway along the 
major semi-axis from equator to base. We assumed a constant aspect ratio $(\gamma)$, equal to the epicardial minor semi-axis length $\left(b_{o}\right)$ divided by the epicardial major semi-axis length $\left(a_{o}\right)$

$\gamma=\frac{b_{o}}{a_{o}}$

which was reported to be 0.67 at end-diastole. For each endocardial volume, we computed the epicardial volume, endocardial and epicardial semi-axis lengths, wall stresses, and wall stretches using the following equations.

The epicardial volume $\left(V_{o}\right)$ was related to the endocardial volume $\left(V_{i}\right)$ by

$V_{o}=V_{i}+V_{\text {wall }}$

where wall volume $\left(V_{\text {wal }}\right)$ was computed from the mass of the left ventricle $\left(m_{L V}\right)$ and density of myocardium $\left(\rho=1.06 \mathrm{~g} / \mathrm{cm}^{3}\right)$ (Fomovsky \& Holmes, 2010)

$V_{\text {wall }}=\frac{m_{L V}}{\rho}$

Assuming a truncated prolate spheroid with truncation factor $T_{f}=0.5$, the epicardial volume was defined as (Streeter \& Hanna, 1973)

$V_{o}=\frac{\pi}{3}\left(2+3 T_{f}-T_{f}{ }^{3}\right) b_{o}{ }^{2} a_{o}=\frac{\pi}{3}\left(2+3 T_{f}-T_{f}{ }^{3}\right) a_{o}{ }^{3} \gamma^{2}$

which allowed us to solve for $a_{o}$ and $b_{o}$.

Assuming confocal endocardial and epicardial surfaces (Holmes, 2004)

$f_{o}^{2}=a_{o}{ }^{2}-b_{o}{ }^{2}=f_{i}^{2}=a_{i}{ }^{2}-b_{i}{ }^{2}$

where $f$ is the focus of an ellipse, we computed the endocardial major and minor semi-axis lengths $\left(a_{i}\right.$ and $\left.b_{i}\right)$ using the equation 
$V_{i}=\frac{\pi}{3}\left(2+3 T_{f}-T_{f}^{3}\right) b_{i}^{2} a_{i}=\frac{\pi}{3}\left(2+3 T_{f}-{T_{f}}^{3}\right)\left({a_{i}}^{2}-{a_{o}}^{2}+b_{o}{ }^{2}\right) a_{i}$

(Eqn. 6.8)

and then calculated the equatorial wall thickness $(h)$ and mid-thickness semi-axis lengths $\left(a_{m}\right.$ and $\left.b_{m}\right)$

$h=b_{o}-b_{i}$

(Eqn. 6.9)

$a_{m}=\frac{1}{2}\left(a_{i}+a_{o}\right)$

$b_{m}=\frac{1}{2}\left(b_{i}+b_{o}\right)$

We then estimated circumferential and longitudinal wall stresses $\left(\sigma_{C C}\right.$ and $\left.\sigma_{L L}\right)$ at the equator of the left ventricular free wall using the formulas (Holmes, 2004)

$\sigma_{C C}=P\left(\frac{b_{m}}{h}\right)\left(1-\frac{b_{m}^{2}}{2 a_{m}^{2}}-\frac{h}{2 b_{m}}+\frac{h^{2}}{8 a_{m}^{2}}\right)$

$\sigma_{C C}=P\left(\frac{b_{m}}{2 h}\right)\left(1-\frac{h}{2 b_{m}}\right)^{2}$

where $P$ is the chamber pressure, and estimated epicardial wall stretches at the equator $\left(\lambda_{C C}\right.$ and $\left.\lambda_{L L}\right)$ as (Holmes, 2004)

$\lambda_{C C}=\frac{b_{o, \text { def }}}{b_{o, r e f}}$

$\lambda_{L L}=\frac{a_{o, \text { def }}}{a_{o, \text { ref }}}$

where the reference semi-axis lengths were those at the unloaded volume $V_{D}^{0}$ (Fig. 6.3b,c). Because we assumed a constant aspect ratio of the left ventricle, the stretches were forced to be equibiaxial throughout inflation. 
Structure-based Constitutive Model

The purpose of coupling a finite element model to the agent-based model was to be able to update strains as the infarct structure changes during healing. We computed constitutive model parameters as a function of collagen area fraction and collagen alignment. We chose a Fungtype constitutive model, given by the strain energy function

$\psi=\frac{1}{2} C\left(e^{Q}-1\right)+\frac{1}{2} K(\ln J)^{2}$

$Q=C_{e}\left(E_{1} \varepsilon_{11}^{2}+E_{2} \varepsilon_{22}^{2}+E_{3} \varepsilon_{33}^{2}+4 G_{12} \varepsilon_{12}^{2}+4 G_{23} \varepsilon_{23}^{2}+4 G_{32} \varepsilon_{32}^{2}\right)$

$J=\operatorname{det} \overline{\bar{F}}$ $\psi$ - strain energy density, $C$ - stress scaling coefficient, $K$ - bulk compressibility, $C_{e}-$ exponential scaling coefficient, $E_{i}$ - stiffness coefficients for direct strains, $G_{i j}$ - stiffness coefficients for shear strains, $\varepsilon_{i j}-$ strain tensor components

where $\vec{e}_{3}$ is along the $\mathrm{z}$-axis (transmural axis), and $\vec{e}_{1}$ and $\vec{e}_{2}$ are in the $\mathrm{x}$-y plane (circumferential-longitudinal plane) along the fiber axis and cross-fiber axis, respectively.

Each stiffness coefficient in $Q$ was assumed to be a weighted sum of the stiffness due to collagen fibers, non-collagen fibers (representing muscle and other fibrous components of myocardium), and an isotropic ground substance, where the weights of the collagen fiber and non-collagen fiber terms were set equal to their area fractions 
$E_{i}=F_{c f} E_{i, c f}+F_{n c f} E_{i, n c f}+\left(1-F_{c f}-F_{n c f}\right) E_{\text {ground }}, \quad i=1,2,3$

$G_{i j}=F_{c f} G_{i j, c f}+F_{n c f} G_{i j, n c f}+\left(1-F_{c f}-F_{n c f}\right) G_{g r o u n d}, i j=12,23,31$

(Eqn. 6.20)

$E_{i}$ - stiffness coefficients for direct strains, $G_{i j}$ - stiffness coefficients for shear strains, $F_{c f}-$ collagen fiber area fraction, $E_{i, c f}$ - collagen fiber stiffness, $F_{n c f}$ - non-collagen fiber area fraction, $E_{i, n c f}-$ non-collagen fiber stiffness, $E_{g r o u n d}-$ ground substance stiffness, $G_{i j, c f}-$ collagen fiber shear stiffness, $G_{i j, n c f}$ - non-collagen fiber shear stiffness, $G_{g r o u n d}$ - ground substance shear stiffness

In order to compute $E_{i, c f}, E_{i, n c f}, G_{i j, c f}$ and $G_{i j, n c f}$, we adopted the approach of Costa $e t$ al. (2001), who related the strain energy based on strain tensor components to a strain energy based on a sum of fiber strains. That is,

$Q=\vec{\sigma} \cdot \vec{\varepsilon}=\vec{\varepsilon}^{T} \overline{\bar{E}} \vec{\varepsilon} \cong \sum_{i=1}^{N_{f}} \sigma_{f} \varepsilon_{f}=E_{f} \sum_{i=1}^{N_{f}} \varepsilon_{f}^{2}=E_{f} \sum_{i=1}^{N_{f}}\left(\vec{u}_{i}^{T} \overline{\bar{\varepsilon}} \vec{u}_{i}\right)^{2}$

$\overline{\bar{E}}=\left[\begin{array}{cccccc}E_{1} & & & & & \\ & E_{2} & & & & \\ & & E_{3} & & & \\ & & & G_{12} & & \\ & & & & G_{23} & \\ & & & & & G_{31}\end{array}\right]$

(Eqn. 6.22)

$\vec{\varepsilon}^{T}=\left[\begin{array}{llllll}\varepsilon_{11} & \varepsilon_{22} & \varepsilon_{33} & 2 \varepsilon_{12} & 2 \varepsilon_{23} & 2 \varepsilon_{31}\end{array}\right]$

(Eqn. 6.23)

$\vec{u}_{i}^{T}=\left[\begin{array}{lll}\cos \phi_{i} \cos \theta_{i} & \cos \phi_{i} \sin \theta_{i} & \sin \phi_{i}\end{array}\right]$

$\overline{\bar{\varepsilon}}=\left[\begin{array}{lll}\varepsilon_{11} & \varepsilon_{12} & \varepsilon_{31} \\ \varepsilon_{12} & \varepsilon_{22} & \varepsilon_{23} \\ \varepsilon_{31} & \varepsilon_{23} & \varepsilon_{33}\end{array}\right]$

$\vec{\sigma}-$ stress vector, $\vec{\varepsilon}-$ strain vector, $\overline{\bar{E}}-$ stiffness matrix, $\sigma_{f}$ - fiber stress, $\varepsilon_{f}$ - fiber strain, $E_{f}-$ fiber stiffness, $N_{f}$ - number of fibers, $\vec{u}$ - unit direction vector, $\overline{\bar{\varepsilon}}$-strain tensor, $\theta$-fiber 
angle in the $\vec{e}_{1}-\vec{e}_{2}$ plane (azimuth) ranging from $-\pi$ to $+\pi, \phi$ - out of plane angle (inclination) ranging from $-\pi / 2$ to $+\pi / 2$

By expanding the expressions $\vec{\varepsilon}^{T} \overline{\bar{E}} \vec{\varepsilon}$ and $E_{f} \sum\left(\vec{u}_{i}{ }^{T} \overline{\bar{\varepsilon}} \vec{u}_{i}\right)^{2}$ and relating terms we find

$$
\begin{aligned}
& E_{1}=E_{f} \sum_{i=1}^{N_{f}} \cos ^{4} \phi_{i} \cos ^{4} \theta_{i} \\
& E_{2}=E_{f} \sum_{i=1}^{N_{f}} \cos ^{4} \phi_{i} \sin ^{4} \theta_{i} \\
& E_{3}=E_{f} \sum_{i=1}^{N_{f}} \sin ^{4} \phi_{i} \\
& G_{12}=E_{f} \sum_{i=1}^{N_{f}} \cos ^{4} \phi_{i} \cos ^{2} \theta_{i} \sin ^{2} \theta_{i} \\
& G_{23}=E_{f} \sum_{i=1}^{N_{f}} \cos ^{2} \phi_{i} \sin ^{2} \phi_{i} \sin ^{2} \theta_{i} \\
& G_{31}=E_{f} \sum_{i=1}^{N_{f}} \cos ^{2} \phi_{i} \sin ^{2} \phi_{i} \cos ^{2} \theta_{i}
\end{aligned}
$$

We used these equations to compute stiffness coefficients for the isotropic ground substance, assuming a uniform spherical distribution of fibers. After normalizing the coefficients by $E_{1, \text { ground }}$ (only the ratio of the coefficients matters because they are all scaled by $C_{e}\left(1-F_{c f}-F_{n c f}\right)$, we obtained the values $E_{\text {ground }}=1$ and $G_{\text {ground }}=1 / 3$.

We then used these equations to compute stiffness coefficients for wrapped normal distributions of fibers in the $\vec{e}_{1}-\vec{e}_{2}$ plane with mean angle along the $\vec{e}_{1}$ axis and mean vector length ranging from zero to one. In this case, we normalized all coefficients by $E_{1, c f}\left(M V L_{c f}=0\right)$ and obtained a look-up table for $E_{1, c f}, E_{2, c f}$, and $G_{12, c f}$ as functions of mean vector length (Fig. 6.4). (E $E_{3, c f}$, $G_{23, c f}$, and $G_{31, c f}$ equal zero because all fibers are in the $\vec{e}_{1}-\vec{e}_{2}$ plane.) We used the mean vector 
length of fibers in normal myocardium $\left(M V L_{n c f}=0.75\right)$ to calculate the non-collagen fiber stiffness terms

$E_{i, n c f}=\beta E_{i, c f}(0.75)$

$G_{i j, n c f}=\beta G_{i j, c f}(0.75)$

where $\beta$ was introduced to allow non-collagen fibers to have a stiffness different from the collagen fiber stiffness.

We used experimental data to determine how the coefficients $C$ and $C_{e}$ should depend on collagen area fraction $\left(F_{c f}\right)$. The ligation infarct study measured the stiffness and collagen area fraction of infarct scars at 1, 2, 3, and 6 weeks after infarction (Fomovsky \& Holmes, 2010). The scars were structurally and mechanically isotropic and their mechanical behavior was modeled with the strain energy function

$\psi=C_{1}\left(I_{c}-3\right)^{2}$

$\psi$ - strain energy density, $C_{1}$ - isotropic stiffness coefficient, $I_{C}$ - first invariant of right Cauchy-Green deformation tensor

For equibiaxial stretch $\left(\lambda_{11}=\lambda_{22}=\lambda\right)$ of an incompressible material, the stresses $\left(\sigma_{i i}\right)$ were $\sigma_{11}=\sigma_{22}=4 C_{1}\left(2 \lambda^{4}-3 \lambda^{2}-\lambda^{-2}+3 \lambda^{-4}-\lambda^{-8}\right)$

We fit the stress equation to the wall stress-wall stretch curves we derived for passive muscle in order to obtain a rough estimate of how the stiffness of passive muscle compared to the stiffness of the ligation infarct scars (Fig. 6.5a). The stiffness coefficient fit to the passive muscle data was two orders of magnitude lower than the lowest stiffness value of the scars. As a whole, the 
stiffness $\left(C_{1}\right)$ versus collagen area fraction $\left(F_{c f}\right)$ data suggested a direct proportionality could adequately describe their relationship (Fig. 6.5b):

$C_{1}=(3440 \mathrm{kPa}) F_{c f}$

In order to determine coefficients in the Fung-type strain energy function that would give mechanical behavior comparable to what was observed for the infarct scars, we fit the stressstrain curve for equibiaxial stretch predicted by the Fung-type strain energy function to the stress-strain curve of the polynomial strain energy function that characterized the infarct scars. The ligation infarcts were structurally and mechanically isotropic, so we set $M V L_{c f}=0$, and we assumed complete degradation of the initial myocardial structure, setting $F_{n c f}=0$. For collagen area fractions ranging from 0.05 to 0.4 (spanning the experimental data), we calculated $C_{1}=(3440 \mathrm{kPa}) F_{c f}$, calculated the polynomial stress-strain curve, and fit the Fung-type stressstrain curve to the polynomial curve (Fig. 6.5c). Over the range of collagen area fractions from 0.05 to 0.4 we found (Fig. 6.5d)

$C_{e}=26.4 F_{c f}+70.2$

$C=(1.85 k P a) F_{c f}$

Finally, we defined the bulk compressibility: $K=10000 C$.

In order to determine coefficients in the Fung-type strain energy function appropriate for normal myocardium, we set $F_{c f}=0.03, F_{n c f}=0.75$, and $M V L_{c f}=0.75$ (initial conditions of the agent-based model), simulated equibiaxial stretch with the finite element model, and fit the predicted stress-stretch curves to the wall stress-wall strain curves that we estimated for passive and active myocardium (Fig. 6.3b,c). For passive myocardium we obtained the values $C=$ 
$0.0941 \mathrm{kPa}, C_{e}=37.1$, and $\beta=0.156$. For active myocardium we obtained the values $C=3.62 \mathrm{kPa}, C_{e}=37.1$, and $\beta=0.374$.

Simulation of Systolic Deformation at Baseline and After Infarction

In general, systolic strains were computed in four steps. First, end-diastolic wall stresses were applied to the model and the deformation tensor $\overline{\bar{F}}_{E D}$ was saved for each element. Second, endsystolic wall stresses were applied to the model and the deformation tensor $\overline{\bar{F}}_{E S}$ was saved for each element. Third, the deformation tensor for the deformation from end-diastole to end-systole was computed as

$\overline{\bar{F}}_{E D \rightarrow E S}=\overline{\bar{F}}_{E S} \overline{\bar{F}}_{E D}^{-1}$

Fourth, the systolic strains were computed as

$\overline{\bar{\varepsilon}}_{E D \rightarrow E S}=\frac{1}{2}\left(\overline{\bar{F}}_{E D \rightarrow E S}{ }^{T} \overline{\bar{F}}_{E D \rightarrow E S}-\overline{\bar{I}}\right)$

For control myocardium (baseline) subjected to end-diastolic wall stresses, each element was assigned the constitutive model parameters obtained for passive myocardium. For control myocardium subjected to end-systolic wall stresses, each element was assigned the constitutive model parameters obtained for active myocardium. The computed systolic strains for control myocardium agreed well with experimental data (Fig. 6.6a).

For acutely cryo-infarcted myocardium subjected to end-diastolic wall stresses, each element was assigned the constitutive model parameters obtained for passive myocardium. For acutely cryo-infarcted myocardium subjected to end-systolic wall stresses, elements were assigned 
constitutive model parameters as a function of their distance away from the center of the infarct. Previously in the agent-based model, we used a cumulative normal distribution to model the strain transition at the infarct border zone. We adopted the same strategy for computing the transition of constitutive model parameters from those of passive myocardium in the center of the infarct to those of active myocardium in the remote tissue. That is,

$K=K_{\text {passive }}+\left(K_{\text {active }}-K_{\text {passive }}\right) \frac{1}{2} \operatorname{erfc}\left[\frac{-(r-R)}{\sigma_{b} \sqrt{2}}\right]$

$K$ - any constitutive model parameter, $r$ - distance from center of infarct, $R$ - infarct radius, $\sigma_{b}$ - standard deviation of the functional border zone, set to $8 \%$ of the infarct diameter (Gallagher $e t$ al., 1986)

The computed systolic strains for acutely cryo-infarcted myocardium agreed well with experimental data (Fig. 6.6a), as did the profile of systolic strain across the infarct border (Fig. 6.6b). Overall, we obtained a prediction of the strain field over the entire ABM domain (Fig. 6.6c,d,e), from which mechanical guidance cue vectors could be computed as described in Chapter 4 (Fig. 6.6f).

\section{Coupled ABM-FEM}

Coupling of the agent-based model (ABM) and finite element model (FEM) was managed entirely within the code of the agent-based model. Coupling was fairly straightforward because our chosen finite element modeling software, FEBio, reads model specifications from XML files and writes model results to TXT files. Using the Matlab function xml_write.m (contributed by 
Jaroslaw Tuszynski), which is designed to convert a data tree stored as a structured array in Matlab into an XML file, we were able to completely control the finite element model through Matlab. We wrote Matlab functions that could build and modify our FEM specification sheet as a structured array, write the specifications to an XML file, execute the FEBio solver, and then read the results from the output TXT files.

Overall, the coupled ABM-FEM ran as follows. During initialization, a function was called that 1. executed the end-diastolic and end-systolic finite element models for acutely cryo-infarcted myocardium, 2. imported the deformation tensors into Matlab matrices, and 3. computed the strain field needed in the ABM to calculate mechanical guidance cues. As the ABM ran, periodically (e.g. after every 24 hours of simulated time) a function was called that 1 . updated constitutive model parameters in the FEM based on the new element-by-element fiber mean vector lengths, mean angles, and collagen area fractions predicted by the ABM; 2. executed the end-diastolic and end-systolic finite element models; 3. imported the deformation tensors into Matlab matrices; and 4. computed and updated the strain field in the ABM.

\section{Effect of Collagen Fiber Structure on Infarct Strains and Mechanical Guidance Cues}

In order to investigate how the mechanical guidance cue might change as the infarct structure changes, we used the finite element model to compute average systolic strains in the infarct region for scars with varying collagen area fraction or varying collagen mean vector length, and we calculated the expected strength of cell alignment $\left(\rho_{m}\right)$ due to the mechanical guidance cue (strain anisotropy). We assumed complete degradation of the pre-existing myocardial structure 
in the infarct region $\left(F_{n c f}=0\right)$. We simulated isotropic scars $\left(M V L_{c f}=0\right)$ with collagen area fractions of $F_{c f}=0.05,0.1$, and 0.4 . We also simulated scars with constant collagen area fraction $\left(F_{c f}=0.4\right)$ and collagen mean vector lengths of $M V L_{c f}=0,0.43$, and 1 (circumferential mean angle).

\section{Effect of Spatial Heterogeneity of Fiber Angles on Infarct Strains and Mechanical Guidance Cues}

In order to test if spatial heterogeneity of strains could promote spatial heterogeneity of collagen fiber alignment, we used the finite element model to compute strains for a slab of tissue replicating - to scale - the fiber structure of a $2 \mathrm{~mm}$ by $2 \mathrm{~mm}$ patch of 3 week old scar from a cryoinfarct located on the anterior wall of the left ventricle (Fig. 6.7). We used a mesh of 50 by 50 elements and calculated element-by-element the expected strength of cell alignment $\left(\rho_{m}\right)$ and axis of cell alignment stimulated by the mechanical guidance cue. We performed this analysis using wall stresses to achieve either 5\% uniaxial strain or 5\% equibiaxial strain. We used $M V L_{c f}=0.75, F_{c f}=0.21$, and $F_{n c f}=0$ uniformly throughout the material, and varied the

fiber angle $\left(M A_{c f}\right)$ element-by-element in accordance with the fiber angles obtained directly from Fiber3 analysis (Karlon et al., 1999) of the $2 \mathrm{~mm}$ by $2 \mathrm{~mm}$ sample scar. 


\subsection{RESULTS AND DISCUSSION}

\section{Effect of Collagen Fiber Structure on Infarct Strains and Mechanical Guidance Cues}

We used the finite element model to investigate how perturbations of collagen area fraction and collagen mean vector length affect systolic strains and mechanical guidance cues within the infarct.

We simulated isotropic scars $\left(M V L_{c f}=0\right)$ with collagen area fractions of $F_{c f}=0.05,0.1$, and 0.4 . Increasing collagen area fraction increased the stiffness of the scar (Fig. 6.8a). Increasing collagen area fraction lowered the longitudinal systolic strain, but had little effect on the circumferential systolic strain, leading to higher strain anisotropy (Fig. 6.8c). The expected strength of cell alignment stimulated by the mechanical guidance cue was relatively insensitive to the change in strain anisotropy (Fig. 6.8e).

These preliminary results suggest that mechanical guidance cues in healing infarcts are insensitive to variations of collagen area fraction observed experimentally. Reliable prediction of the effect of increasing collagen area fraction on the mechanical guidance cue will depend on accurate modeling of the infarct geometry, the loads acting on the infarct, and the constitutive properties of infarct scar.

We simulated scars with constant collagen area fraction $\left(F_{c f}=0.4\right)$ and collagen mean vector lengths of $M V L_{c f}=0,0.43$, and 1 (circumferential mean angle). Increasing collagen mean 
vector length increased the mechanical anisotropy of the scar (Fig. 6.8b). Increasing collagen mean vector length lowered the circumferential systolic strain and raised the longitudinal systolic strain (Fig. 6.8d), leading to lower strain anisotropy and a weaker mechanical guidance cue (Fig. 6.8f).

Because the loads acting on the infarct caused predominantly circumferential systolic stretch, increasing alignment of fibers along the circumferential (fiber) axis acted to oppose this stretch and reduced the strain anisotropy. This illustrates a potentially important negative feedback process. An increase in fiber alignment will decrease the strength of the mechanical guidance cue, which lowers the strength of cell alignment, which then decreases the strength of fiber alignment. Currently, this effect is predicted to be very weak. However, this may be because we are under-weighting the contribution of collagen fibers to the stiffness coefficients of the constitutive model.

\section{Effect of Spatial Heterogeneity of Fiber Angles on Infarct Strains and Mechanical Guidance Cues}

We observed that 3 week old cryoinfarct scars have a structure that is spatially heterogeneous, consisting of domains on the order of hundreds of microns in size, where collagen fibers are highly aligned within each domain, but weakly aligned from domain to domain. We reasoned that our agent-based model of infarct healing may have been unable to predict these structures because of the static strain field we implemented in the prior model. We created a finite element 
model simulating a heterogeneous fiber structure in order to test if spatial heterogeneity of strains could support spatial heterogeneity of collagen fiber alignment.

We simulated equibiaxial stretch or uniaxial stretch of a $2 \mathrm{~mm}$ by $2 \mathrm{~mm}$ sheet of 3 week old cryoinfarct scar with a realistic spatial arrangement of fiber angles (Fig. 6.7). For equibiaxial stretch and uniaxial stretch, the strains were spatially heterogeneous (Fig. 6.9). For equibiaxial stretch, the expected strength of cell alignment and axis of cell alignment were spatially heterogeneous, and the axis of cell alignment was generally perpendicular to the local fiber axis (Fig. 6.10a). For uniaxial stretch, the strengths and axes of cell alignment displayed less spatial heterogeneity because of the globally anisotropic strain pattern (Fig. 6.10b).

These results suggest that spatial heterogeneity of fiber angles cannot be explained by spatial heterogeneity of fiber strains. For both equibiaxial stretch and uniaxial stretch, the expected axes of cell alignment were not parallel to the fiber axes. Consequently, the mechanical guidance cue was expected to stimulate remodeling of collagen fibers away from the current fiber axis, which would tend to dissipate the initial spatial heterogeneity of fiber angles. This raises an important point, which is that we do not know whether spatial heterogeneity of the collagen fiber structure of infarct scar is present early after infarction and then fades over time, is absent early after infarction and then develops over time, or is a ubiquitous feature of infarct scar. Experimental measurement of spatial heterogeneity at multiple time points after infarction would provide critical information for developing hypotheses that can be tested with the model. 


\section{Limitations and Future Work}

The main limitation of the coupled ABM-FEM is its slow processing time. One of the great advantages of the ABM was its fast processing time - simulation of 6 weeks of infarct healing took about 6 hours. The FEM takes about 15 minutes to compute systolic strains with the current mesh, which roughly triples the processing time if strains are updated after every 24 hours of simulated time. Ideally, a more refined mesh would be used (the current convergence tolerance of $5 \%$ is fairly loose), which would increase the processing time further.

The FEM very simplistically simulates end-diastolic and end-systolic loading of a sheet of myocardium. Although this model adequately served as a proof-of-concept and allowed some preliminary analysis, it lacks important features, such as loading due to cavity pressure, a realistic three dimensional geometry, a transmural gradient of myofiber orientations, and hemodynamic shifts post-infarction. More sophisticated finite element models of the heart already exist, such as Continuity (Kerckhoffs et al., 2012). Future work could include developing a scheme for prescribing regional variations of material properties in Continuity in order to simulate an infarct and its evolving mechanical properties.

Our scheme for updating constitutive model parameters based on the collagen area fraction and fiber orientation distribution of the scar requires experimental validation. Although we had experimental data that motivated calculating some constitutive model parameters from collagen area fraction, the ratio of the stiffness of collagen fibers to the stiffness of the ground substance $\left(E_{i, c f} / E_{\text {ground }}\right)$ was undetermined. A higher stiffness of collagen fibers relative to the stiffness 
of the ground substance could amplify the sensitivity of infarct strains to variations of collagen area fraction and collagen mean vector length.

\subsection{CONCLUSION}

We developed a finite element model of a circular infarct embedded in a sheet of myocardium subjected to circumferential and longitudinal wall stresses. We developed a scheme for updating the constitutive properties of the infarct as its structure (collagen area fraction and fiber mean vector length) changes over time after infarction. We developed a method of coupling the finite element model to our agent-based model of infarct healing, such that the structure predicted by the agent-based model was used to compute the constitutive properties in the finite element model, and the strains predicted by the finite element model were used to compute mechanical guidance cues in the agent-based model. The finite element model reproduced systolic strains for normal myocardium and acutely cryo-infarcted myocardium. We observed the possibility of a negative feedback loop whereby the strength of the mechanical guidance cue reacts to counteract any change in the strength of fiber alignment. However, overall, the mechanical guidance cue was insensitive to experimentally observed variations of the infarct collagen structure, possibly because the stiffness of collagen fibers relative to the isotropic ground substance was too low. Finally, we found that alignment of cells in response to mechanical guidance cues and subsequent collagen fiber remodeling would tend to dissipate, rather than reinforce spatial heterogeneities in the collagen fiber structure of infarct scar. These preliminary studies highlight the utility of a coupled ABM-FEM for studying infarct healing and motivate future work, such as mechanical characterization of anisotropic scars, measurement of spatial 
heterogeneity at multiple time points after infarction, and integration of the agent-based model with a finite element model of the heart. 


\subsection{FIGURES AND TABLES}

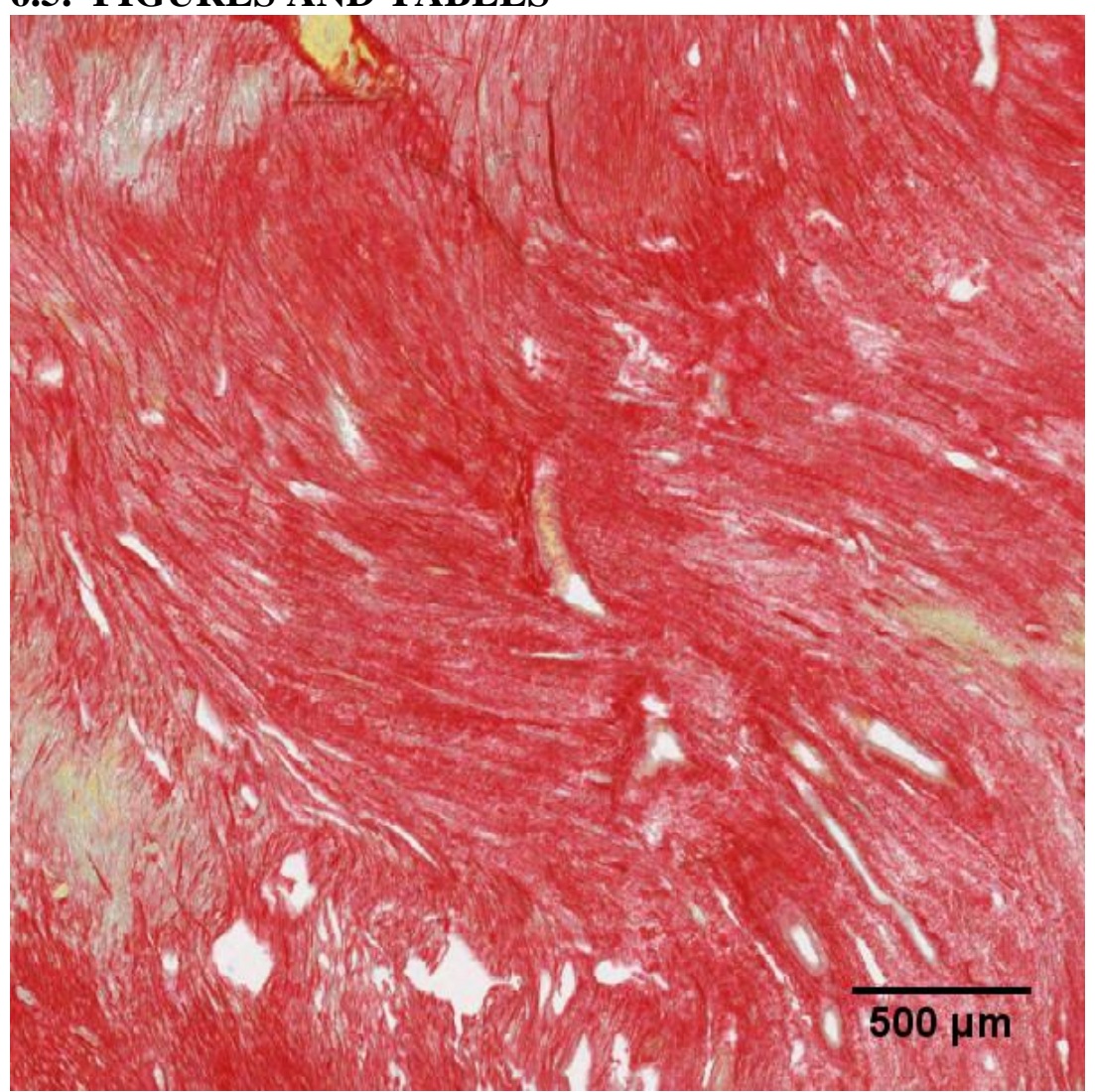

Figure 6.1. Sample of cryoinfarct scar tissue illustrating spatial heterogeneity of collagen fiber alignment. 
A

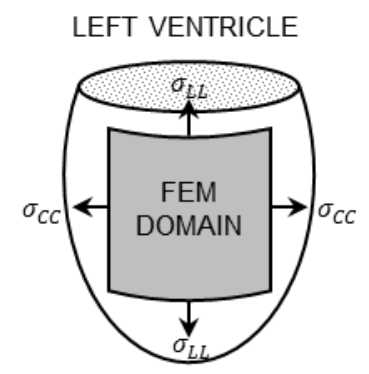

$\mathrm{C}$

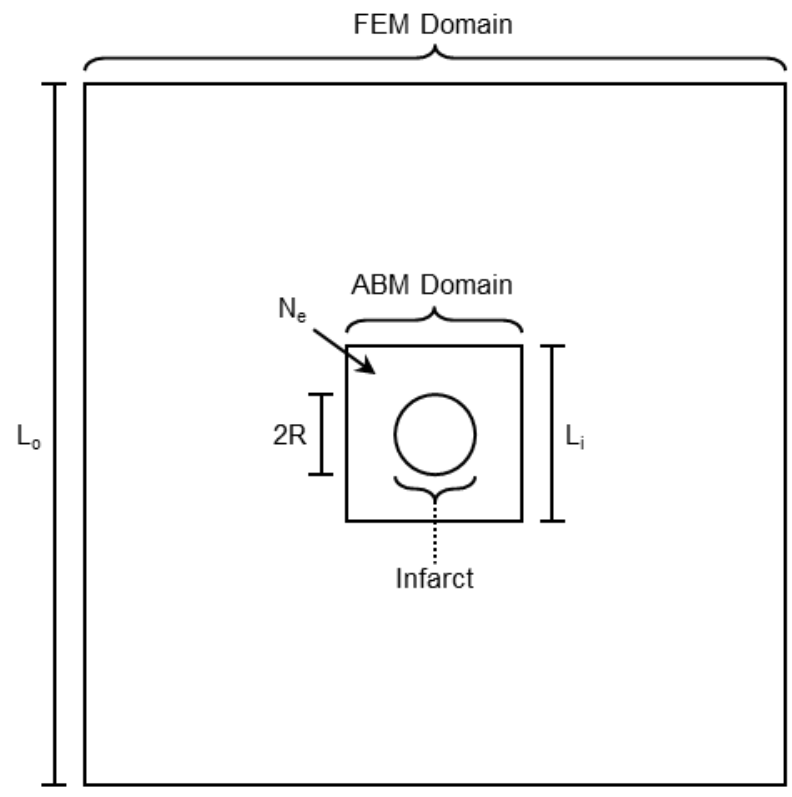

B

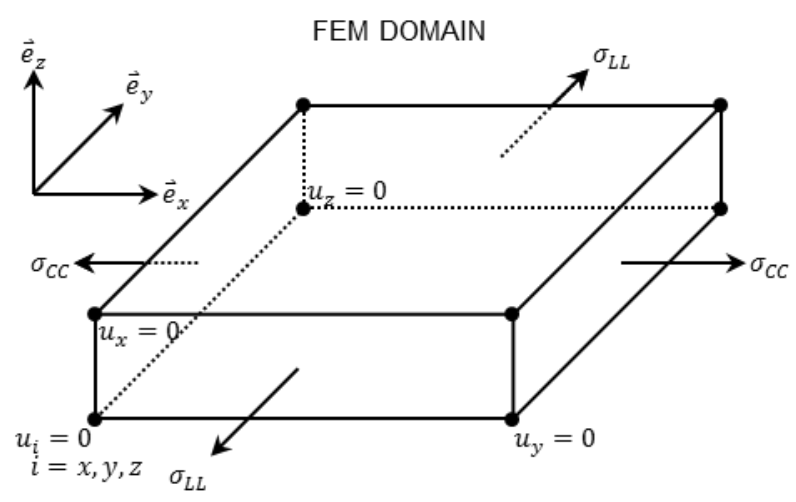

$\mathrm{D}$

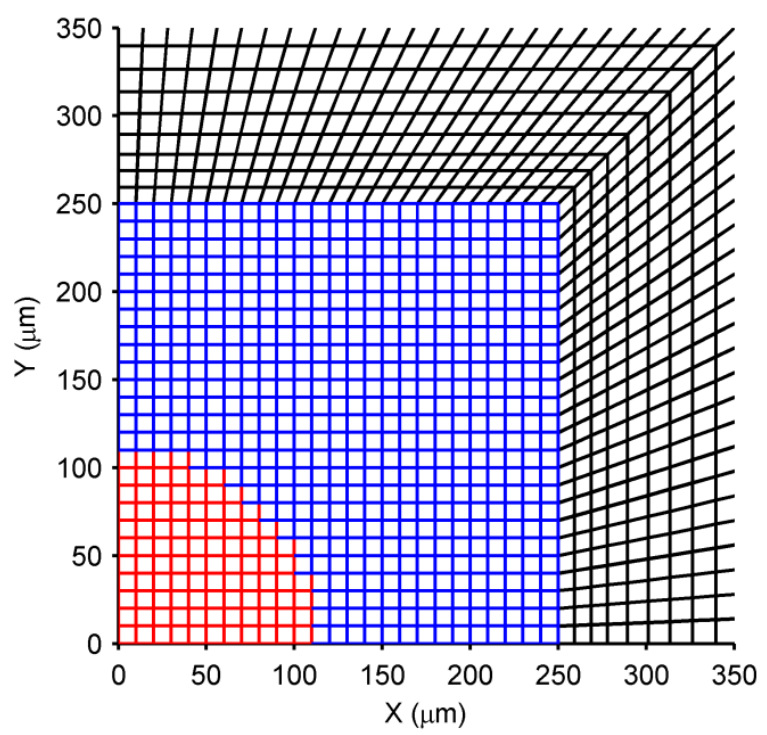

Figure 6.2. Finite element model geometry, boundary conditions, and mesh. A. The finite element model simulated a square sheet of myocardium cut from the anterior wall of the left ventricle, acted upon by circumferential and longitudinal wall stresses $\left(\sigma_{C C}\right.$ and $\left.\sigma_{L L}\right)$. B. The sheet of myocardium was modeled as a thin, square slab of material. We used a Cartesian coordinate system with the $\mathrm{x}$-axis defined as the circumferential axis, the y-axis defined as the longitudinal axis, and the z-axis defined as the transmural axis. Wall stresses acting in the outward normal direction were applied to the side faces of the slab. Rigid body translation and rigid body rotation were prevented by setting displacements of the nodes at the corners of the slab equal to zero where indicated (e.g. $u_{i}=0$ ). C. The FEM domain was set-up such that the size of an inner square region was matched to the size of the ABM $\left(L_{i}=250 \mu m\right)$. The $\mathrm{ABM}$ domain contained a circular infarct region with radius matched to that of the $\mathrm{ABM}(R=113 \mu \mathrm{m})$. The size of the whole FEM domain $\left(L_{o}=2000 \mu \mathrm{m}\right)$ was chosen to ensure that the deformations within the infarct region were insensitive to the location of the outermost boundary. D. Within the ABM domain, the model was divided into a 50 by 50 element mesh. Elements were classified as belonging to the infarct (red) or to the surrounding muscle (blue). Outside the ABM domain (black), elements were expanded from the edge of the ABM domain to the edge of the FEM domain in a butterfly pattern. The number of elements in the infarct region $\left(N_{e}=2500\right)$ was determined by convergence of the deformations within the infarct region to $5 \%$. 
A

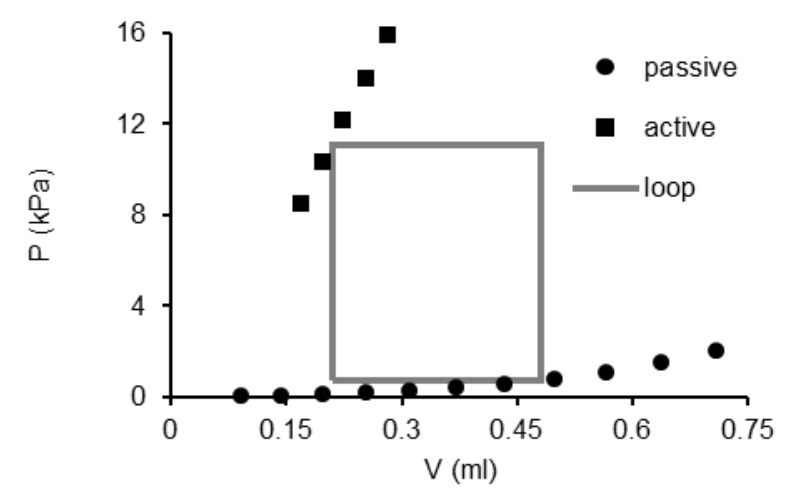

$\mathrm{C}$

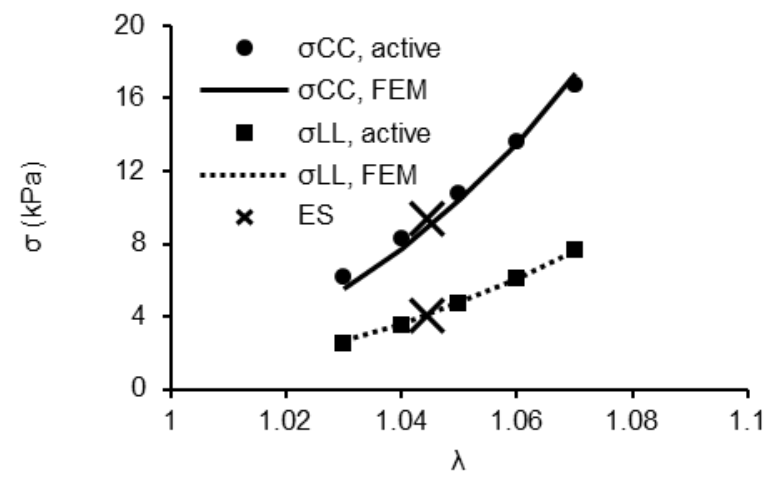

B

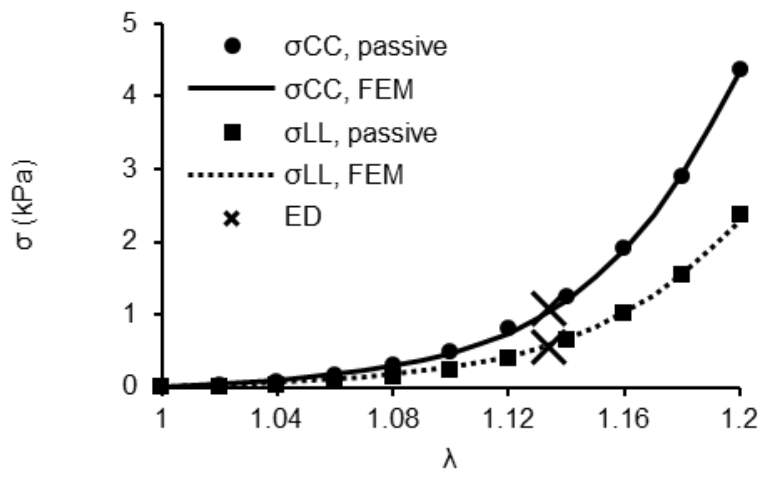

Figure 6.3. Fitting mechanical behavior of passive and active myocardium. We obtained hemodynamic measurements of control rat hearts reported in Fomovsky \& Holmes (2010). We assumed the left ventricle had the geometry of a truncated prolate ellipsoid with a constant major to minor axis length ratio throughout inflation, which allowed measurements of pressure and volume to be converted to estimates of wall stresses in response to equibiaxial stretch. We then simulated equibiaxial stretch of normal myocardium with the finite element model and iteratively solved for the coefficients of the constitutive model that could match the estimated stress-strain curves. A. ESPVR and EDPVR from Fomovsky \& Holmes (2010). B. For passive muscle, we derived stress-strain curves for equibiaxial stretch from EDPVR data $\left(\sigma_{c c \text {,passive }}\right.$ and $\left.\sigma_{l l, p a s s i v e}\right)$. We found coefficients of the constitutive model that adequately reproduced the estimated stressstrain curves $\left(\sigma_{c C, F E M}\right.$ and $\left.\sigma_{l l, F E M}\right)$. "X" marks indicate the estimated stresses and strains at end diastole. C. For active muscle, we derived stress-strain curves for equibiaxial stretch from ESPVR data $\left(\sigma_{c c \text {,active }}\right.$ and $\left.\sigma_{\text {ll,active }}\right)$. We found coefficients of the constitutive model that adequately reproduced the estimated stress-strain curves $\left(\sigma_{c c, F E M}\right.$ and $\left.\sigma_{l l, F E M}\right)$. " $\mathrm{X}$ " marks indicate the estimated stresses and strains at end systole. 
A

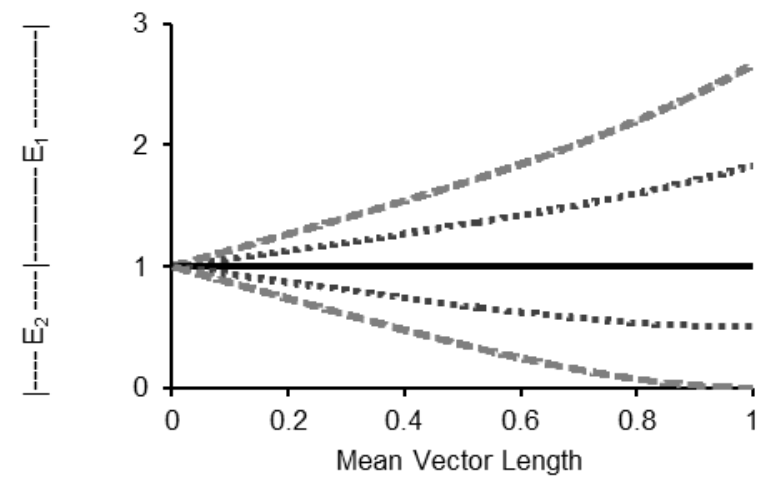

B

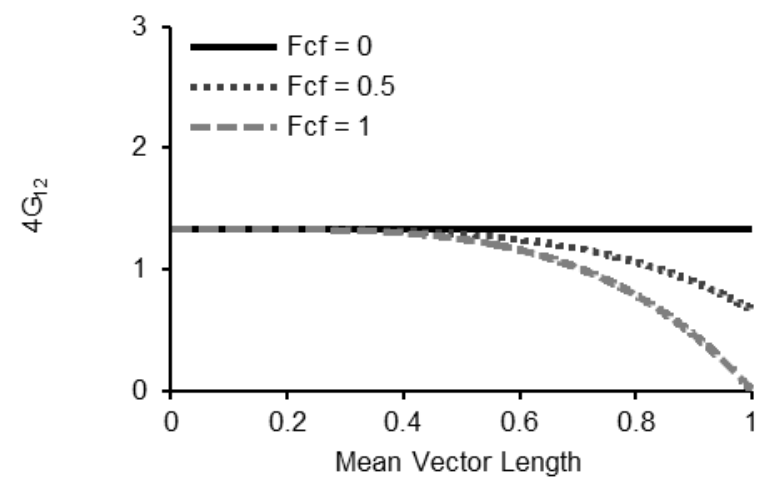

Figure 6.4. Computation of stiffness coefficients from fiber structure. We used the approach of Costa et al. (2001) to compute stiffness coefficients from known fiber structure for use in a Fung-type strain energy function. The stiffness coefficients were computed by integrating certain trigonometric functions (Eqn. X to Eqn. Y) over the fiber orientation distribution given the fiber mean vector length, where the fiber distribution was assumed to have the shape of a wrapped normal distribution. We assumed the existence of an isotropic ground substance, which we represented as a uniform spherical fiber distribution. We calculated the stiffness coefficients as a weighted sum of the stiffnesses of the isotropic ground substance, the collagen fiber distribution predicted by the agent-based model, and the non-collagen fiber distribution (accounting for muscle and other fibrous material initially present in infarcted myocardium) predicted by the agent-based model. Weight factors were set equal to the fiber area fractions, with the ground substance accounting for any remaining fraction. We computed a look-up table of fiber $\left(\mathrm{E}_{1}\right)$, cross-fiber $\left(\mathrm{E}_{2}\right)$, and shear $\left(\mathrm{G}_{12}\right)$ stiffness coefficients over the full range of fiber mean vector lengths. Results are shown for a non-collagen area fraction of $F_{n c f}=0$ and collagen area fractions of $F_{c f}=0,0.5$, and 1 . As the strength of fiber alignment increased, stiffness in the fiber direction increased (A), stiffness in the cross-fiber direction decreased (A), and the shear stiffness decreased (B). 
A

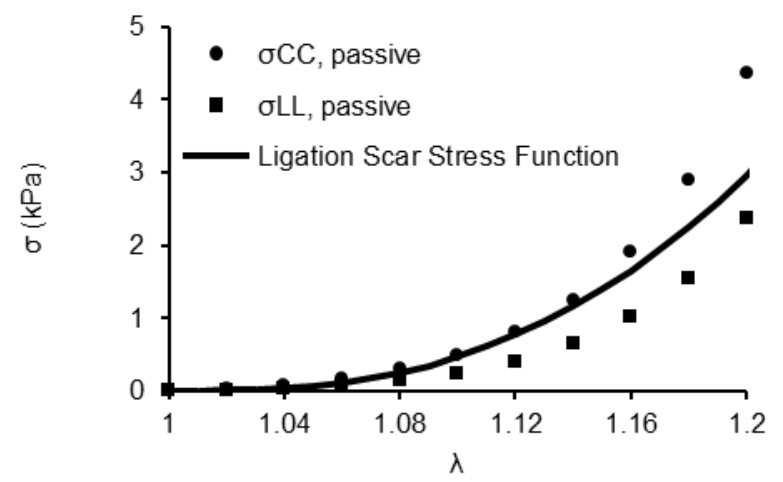

$\mathrm{C}$

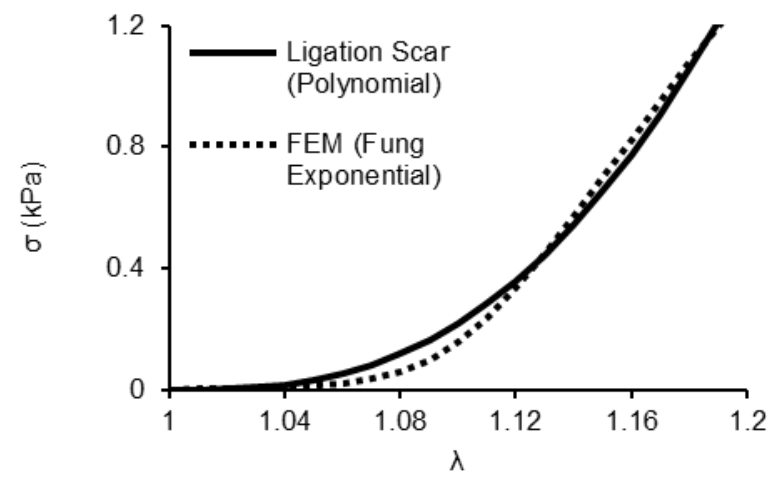

B

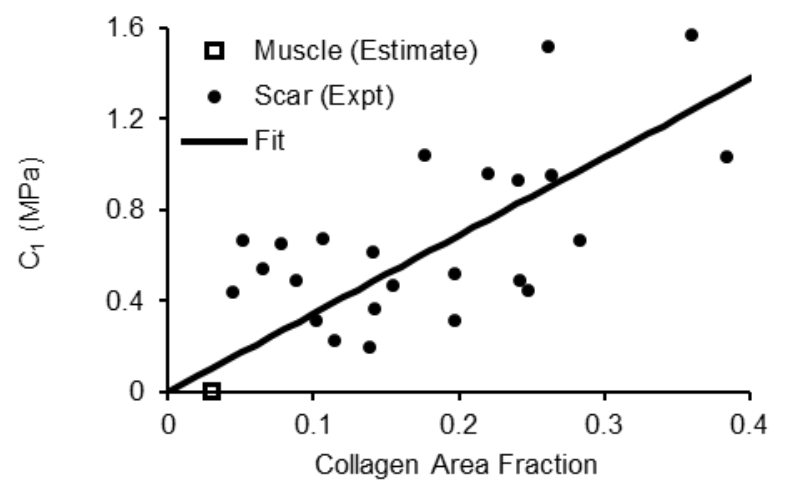

$\mathrm{D}$

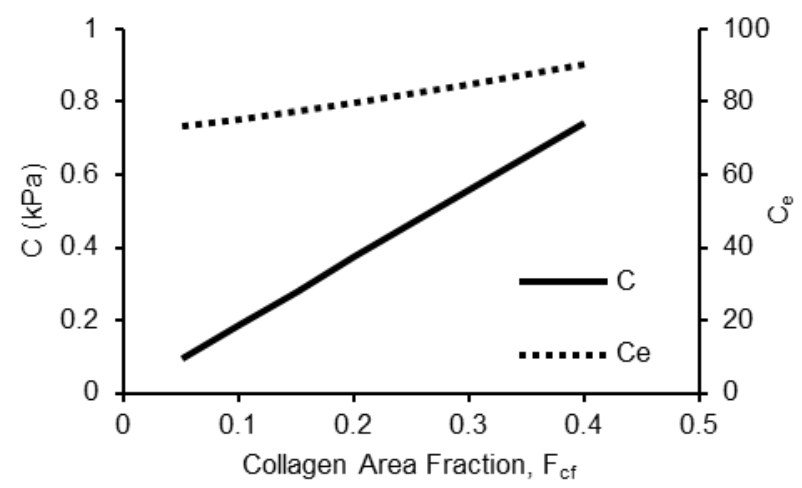

Figure 6.5. Stiffness coefficient scaling. We determined an equation for scaling stiffness coefficients in the constitutive model as a function of collagen area fraction. Fomovsky \& Holmes (2010) determined stiffness coefficients of rat ligation infarct scars from biaxial mechanical tests and also measured collagen area fraction. A. In order to estimate how the stiffness of passive myocardium compares to the stiffness of infarct scar, we fit our estimated stress-strain curves for passive muscle to the stress-strain relationship of the strain energy function that was used to characterize the infarct scars. The stiffness coefficient of passive muscle was $2 \mathrm{kPa}$, roughly two orders of magnitude lower than the stiffness coefficients of infarct scar, which were measured from 1 to 6 weeks after infarction. B. A plot of all stiffness coefficients against collagen area fraction (Fomovsky \& Holmes, 2010) suggested a simple proportionality factor could reasonably relate stiffness to collagen area fraction: $C_{1}=$ $(3440 \mathrm{kPa}) F_{c f}$. C and D. In order to determine coefficients in our Fung-type strain energy function that would give mechanical behavior comparable to what was observed for the infarct scars, we fit the stress-strain curve for equibiaxial stretch predicted by the Fung-type strain energy function to the stress-strain curve predicted by the polynomial strain energy function that characterized the infarct scars. The ligation infarcts were structurally and mechanically isotropic, so we set $M V L_{c f}=0$, and we assumed complete degradation of the initial myocardial structure, setting $F_{n c f}=0$. For collagen area fractions ranging from 0.05 to 0.4 , we used the correlation in plot $\mathrm{B}$ to calculate $C_{1}$ and then calculated the polynomial stress-strain curve (Ligation Scar). By fitting the exponential stress-strain curve (FEM) to the polynomial curve over the range of collagen area fractions $\left(F_{c f}\right)$, we found $C_{e}=26.4 F_{c f}+70.2$ and $C=$ $(1.85 \mathrm{kPa}) F_{c f}$. 
A

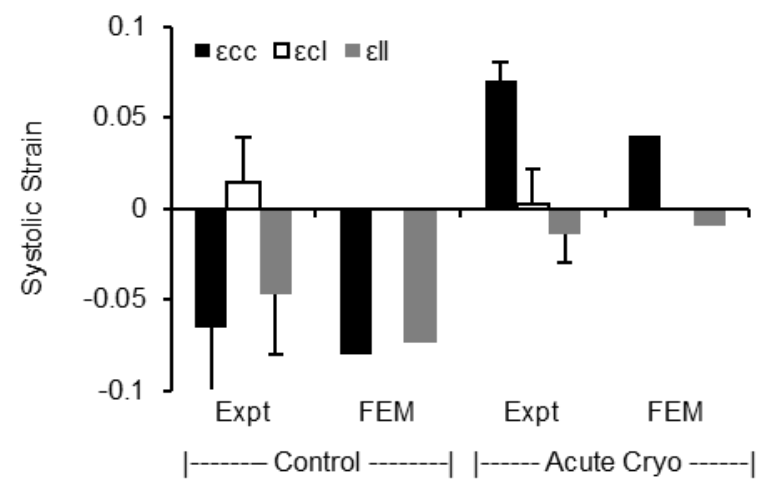

$\mathrm{C}$

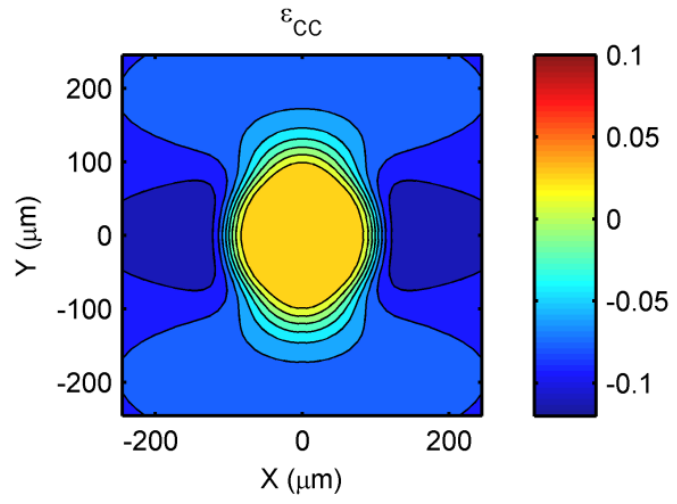

$\mathrm{E}$

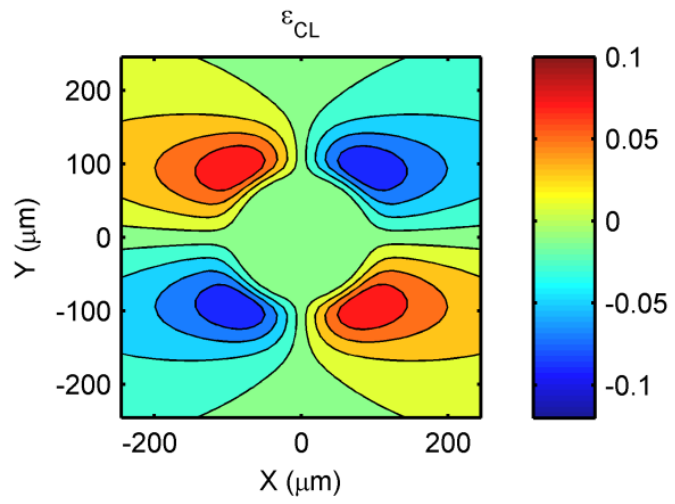

B

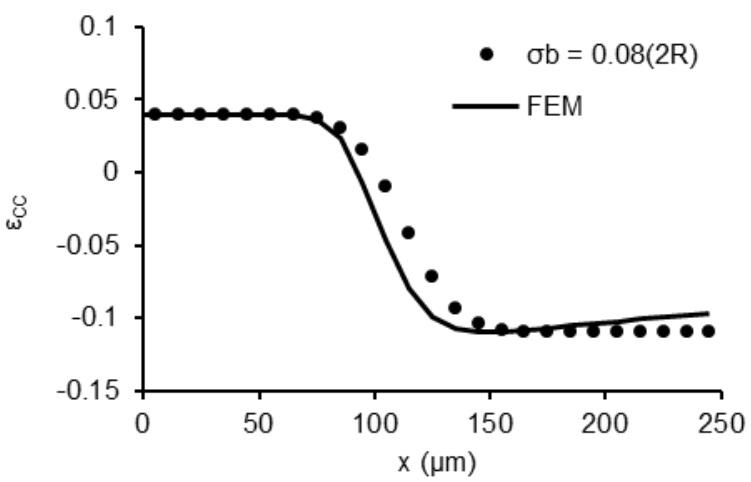

$\mathrm{D}$
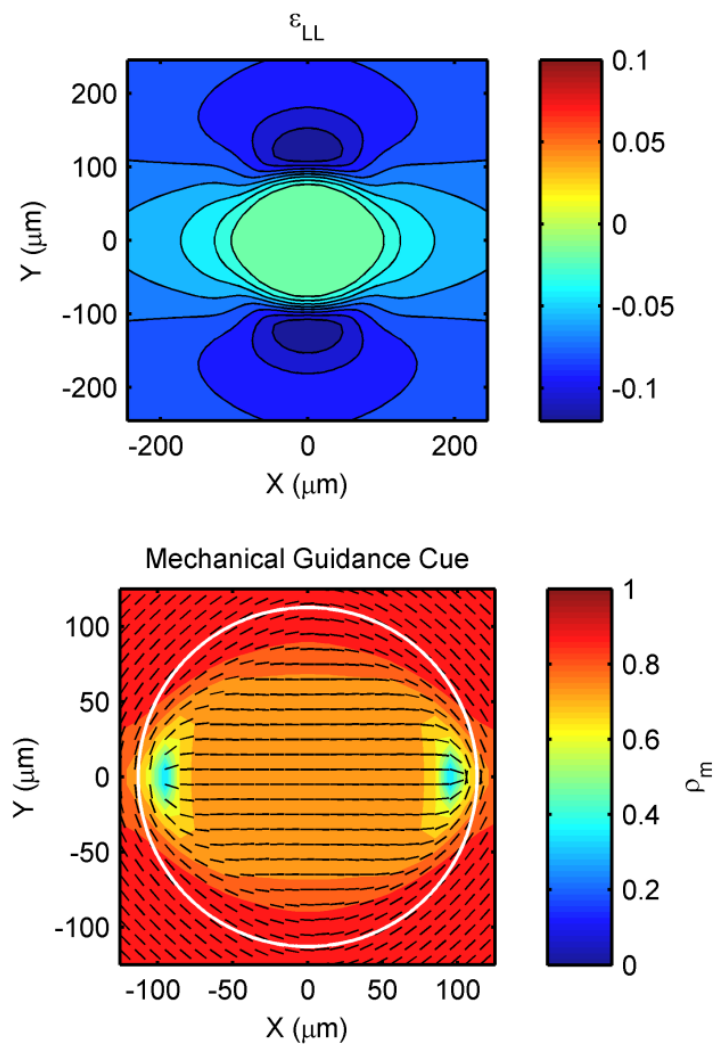

Figure 6.6. Simulated systolic strains. We applied end diastolic wall stresses to the finite element model using material coefficients for passive muscle in order to compute the deformed state at end diastole. We then applied end systolic wall stresses to the finite element model using material coefficients for active muscle in order to compute the deformed state at end systole. Systolic strains were computed between the end diastolic and end systolic states. The simulations were then repeated with the material coefficients of the infarct region at end systole changed to the values for passive muscle in order to compute the systolic strains acutely after infarction. A. The finite element model reproduced the experimentally measured systolic strains for normal muscle (control) and acutely ischemic muscle (acute cryo) (Fomovsky et al., 2012). B. For the acute cryoinfarction case, the width of the functional border zone, measured as the width of the transition of strain from the center of the infarct to the remote region, was similar to the width we intended to match based on experimental data (8\% of the infarct diameter) (Gallagher et al., 1986). C, D, and E. Contour plots of systolic strains immediately after infarction. F. Mechanical guidance cue immediately after infarction. Dashes indicate the axis of cell alignment and color indicates the expected strength of cell alignment. 

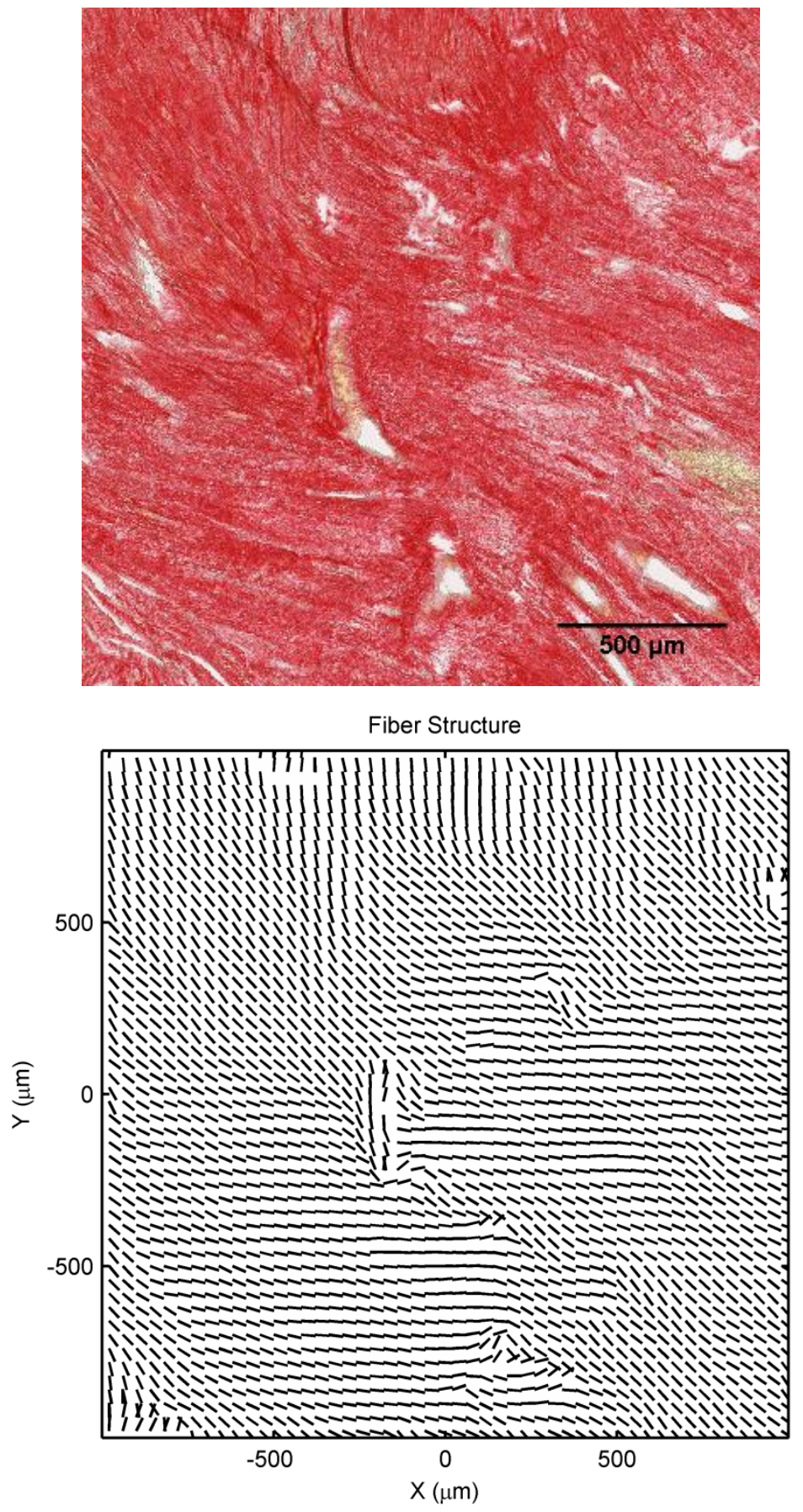

Figure 6.7. Detailed fiber structure of cryoinfarct scar. We selected for detailed structural analysis a $2 \mathrm{~mm}$ by $2 \mathrm{~mm}$ region of scar from a tissue section at $30 \%$ transmural depth into a 3 week old cryoinfarct located on the anterior wall of the left ventricle. The tissue section was stained with picrosirius red, and a high resolution image $(0.5 \mu \mathrm{m} / \mathrm{px})$ was captured using the Scanscope slide scanner. A $2 \mathrm{~mm}$ by $2 \mathrm{~mm}$ region of scar was cropped from the image and filtered to retain only red colored pixels corresponding to picrosirius red stained collagen fibers. Fiber angles were measured using the Fiber3 algorithm (Karlon et al., 1999) with a sub-region size of $20 \mu \mathrm{m}$. Fiber angles were then fit by smoothed interpolation to a 50 by 50 element mesh for finite element analysis (shown above). 
A

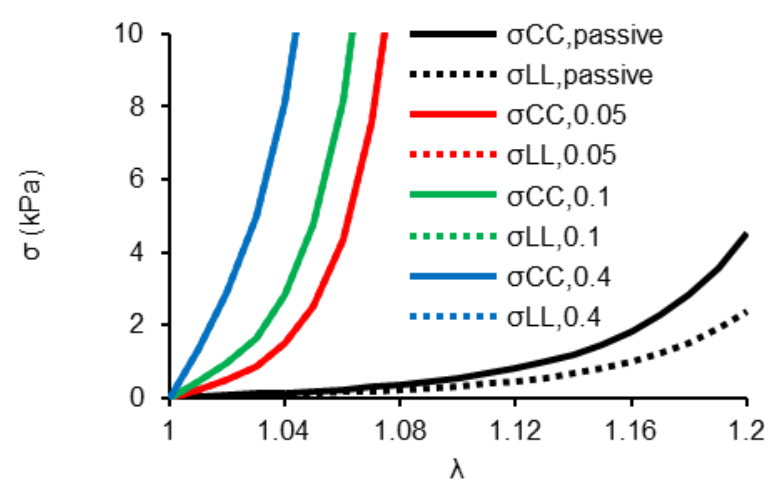

$\mathrm{C}$

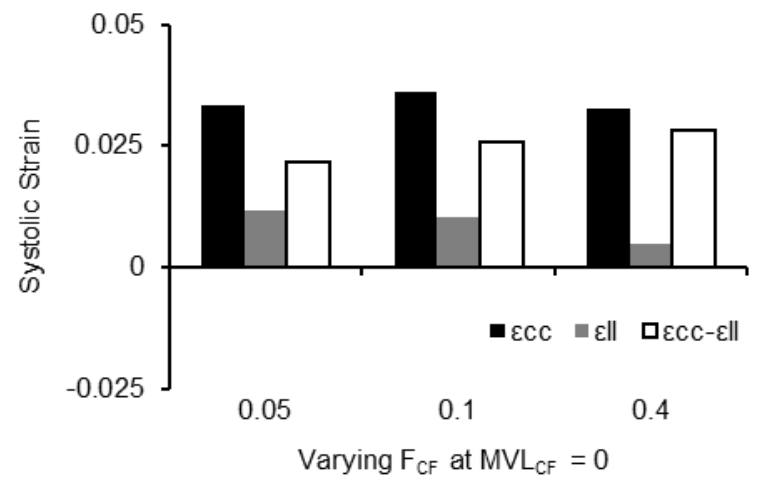

$\mathrm{E}$

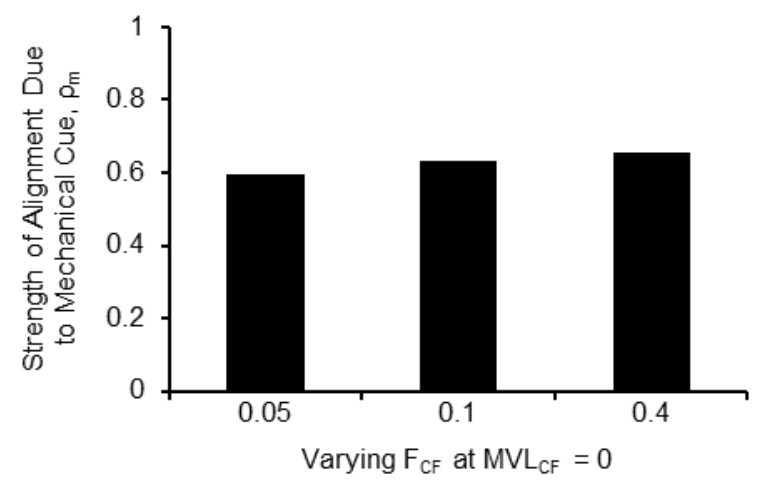

B Effect of Collagen Mean Vector Length

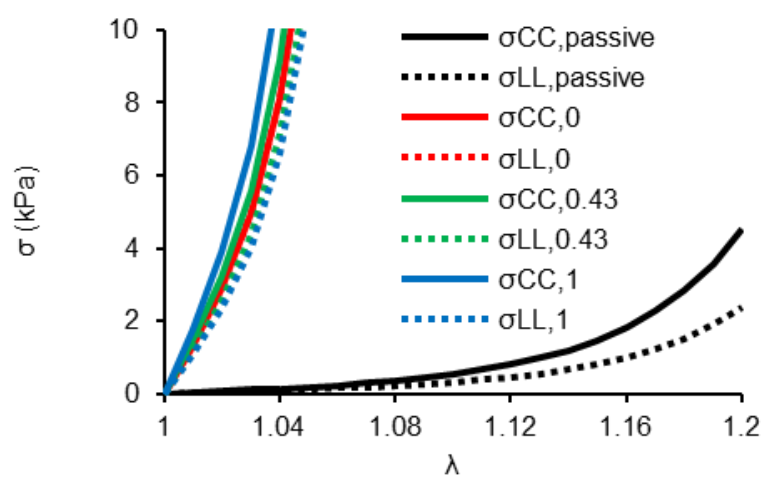

$\mathrm{D}$

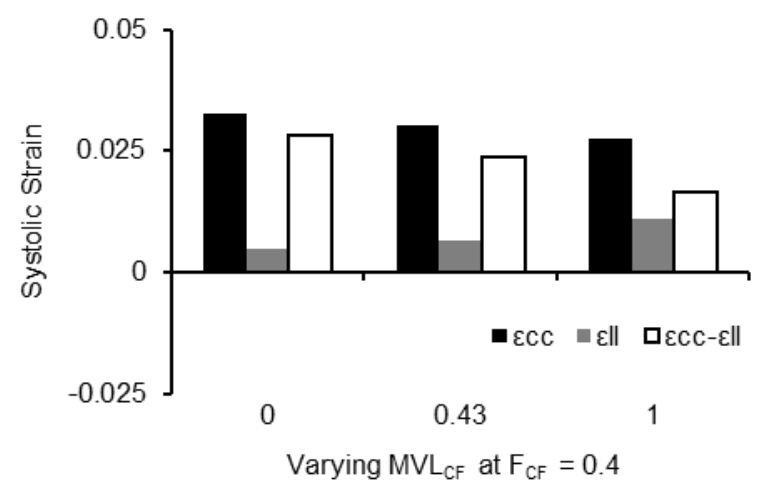

$\mathrm{F}$

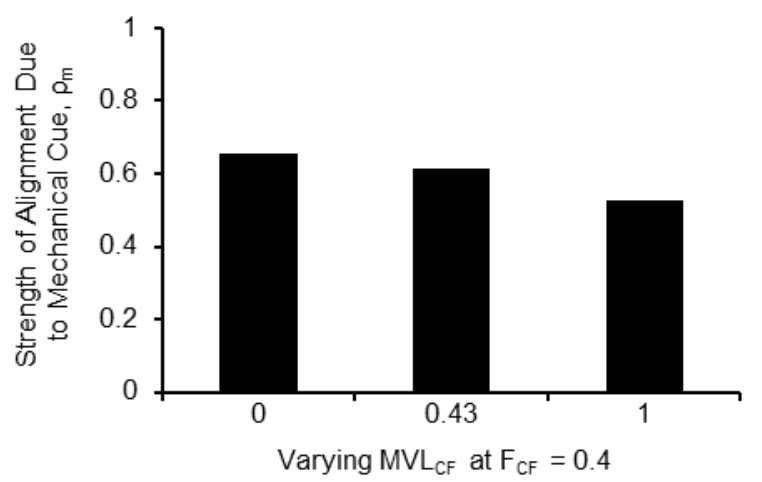

Figure 6.8. We used the FEM to investigate how perturbations of collagen area fraction and strength of collagen alignment affect systolic strains and mechanical guidance cues within the infarct. LEFT: We simulated isotropic scars $\left(M V L_{c f}=0\right)$ with collagen area fractions of $F_{c f}=0.05,0.1$, and 0.4. A. Increasing collagen area fraction increased the stiffness of the scar, as indicated by the stress curves for equibiaxial stretch. C. Increasing collagen area fraction lowered the longitudinal systolic strain $\left(\varepsilon_{L L}\right)$, but had little effect on the circumferential systolic strain $\left(\varepsilon_{C C}\right)$, leading to higher strain anisotropy $\left(\varepsilon_{C C}-\varepsilon_{L L}\right)$. E. The strength of alignment due to the mechanical cue $\left(\rho_{m}\right)$ was relatively insensitive to the change in strain anisotropy. RIGHT: We simulated scars with constant collagen area fraction $\left(F_{c f}=0.4\right)$ and collagen mean vector lengths of $M V L_{c f}=0,0.43$, and 1 (circumferential mean angle). B. Increasing collagen mean vector length increased the mechanical anisotropy of the scar, as indicated by separation of the circumferential and longitudinal stresses predicted for equibiaxial stretch. D. Increasing collagen mean vector length lowered the circumferential systolic strain and raised the longitudinal systolic strain, leading to lower strain anisotropy and a weaker mechanical guidance cue $(\mathbf{F})$. 
A

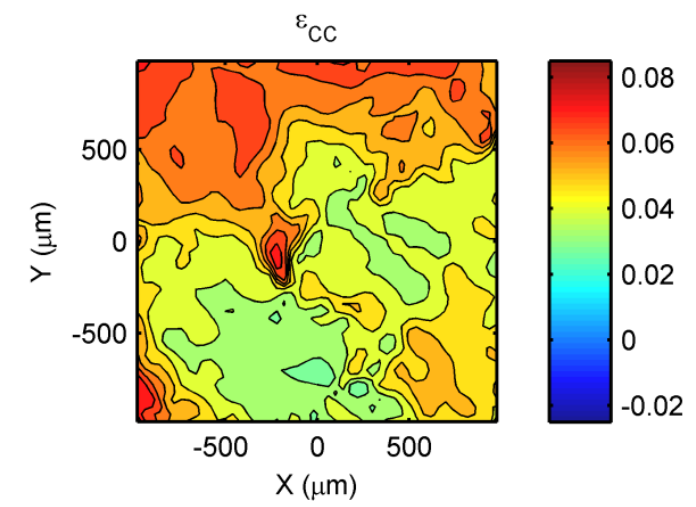

C

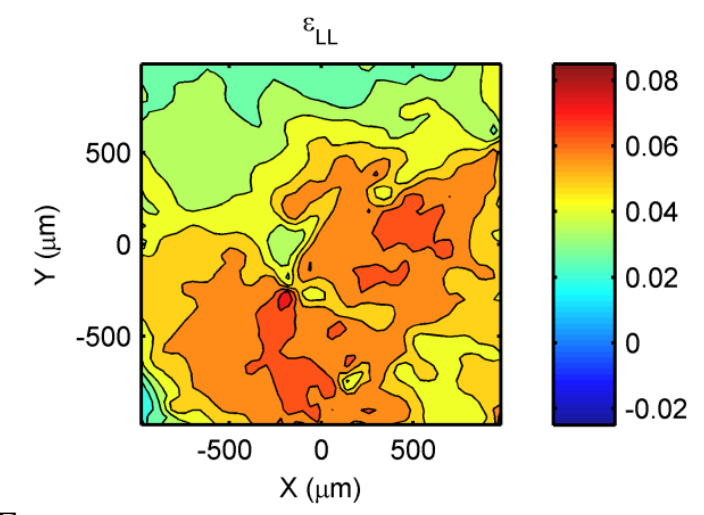

$\mathrm{E}$

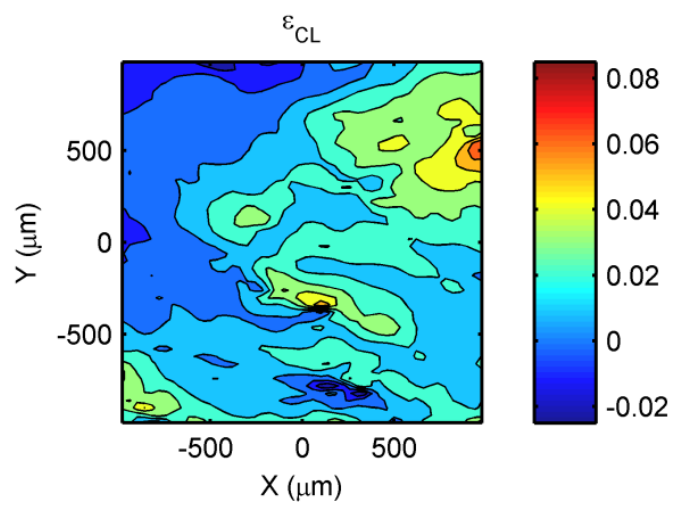

B Uniaxial Strain

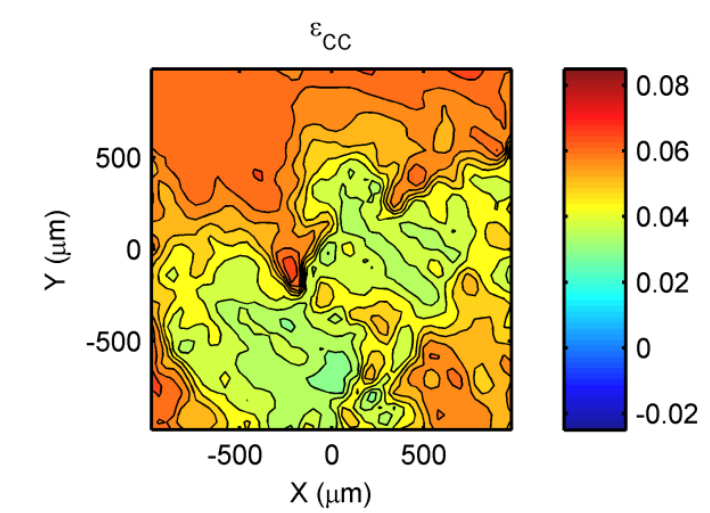

$\mathrm{D}$

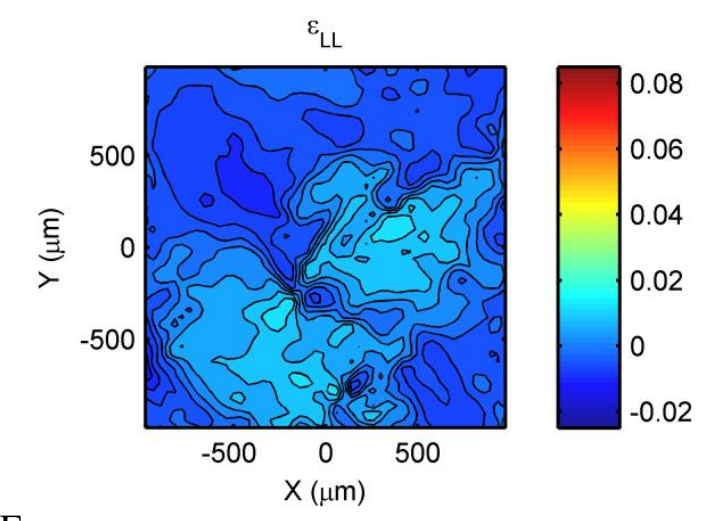

$\mathrm{F}$

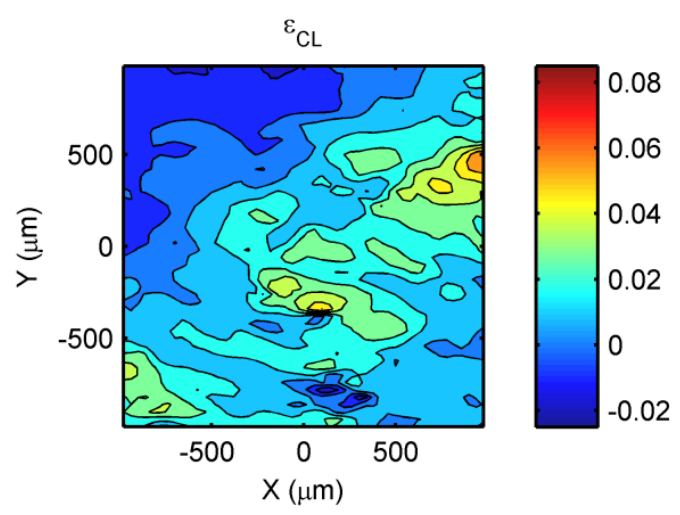

Figure 6.9. Effect of spatial heterogeneity of fiber angles on infarct strains. We simulated equibiaxial stretch (left panels) or uniaxial stretch (right panels) of a $2 \mathrm{~mm}$ by $2 \mathrm{~mm}$ sheet of 3 week old cryoinfarct scar with a realistic spatial arrangement of fiber angles (Fig. 6.6). For equibiaxial stretch and uniaxial stretch, the spatially heterogeneous fiber angles were predicted to cause spatially heterogeneous strains throughout the scar. 
A Equibiaxial Strain

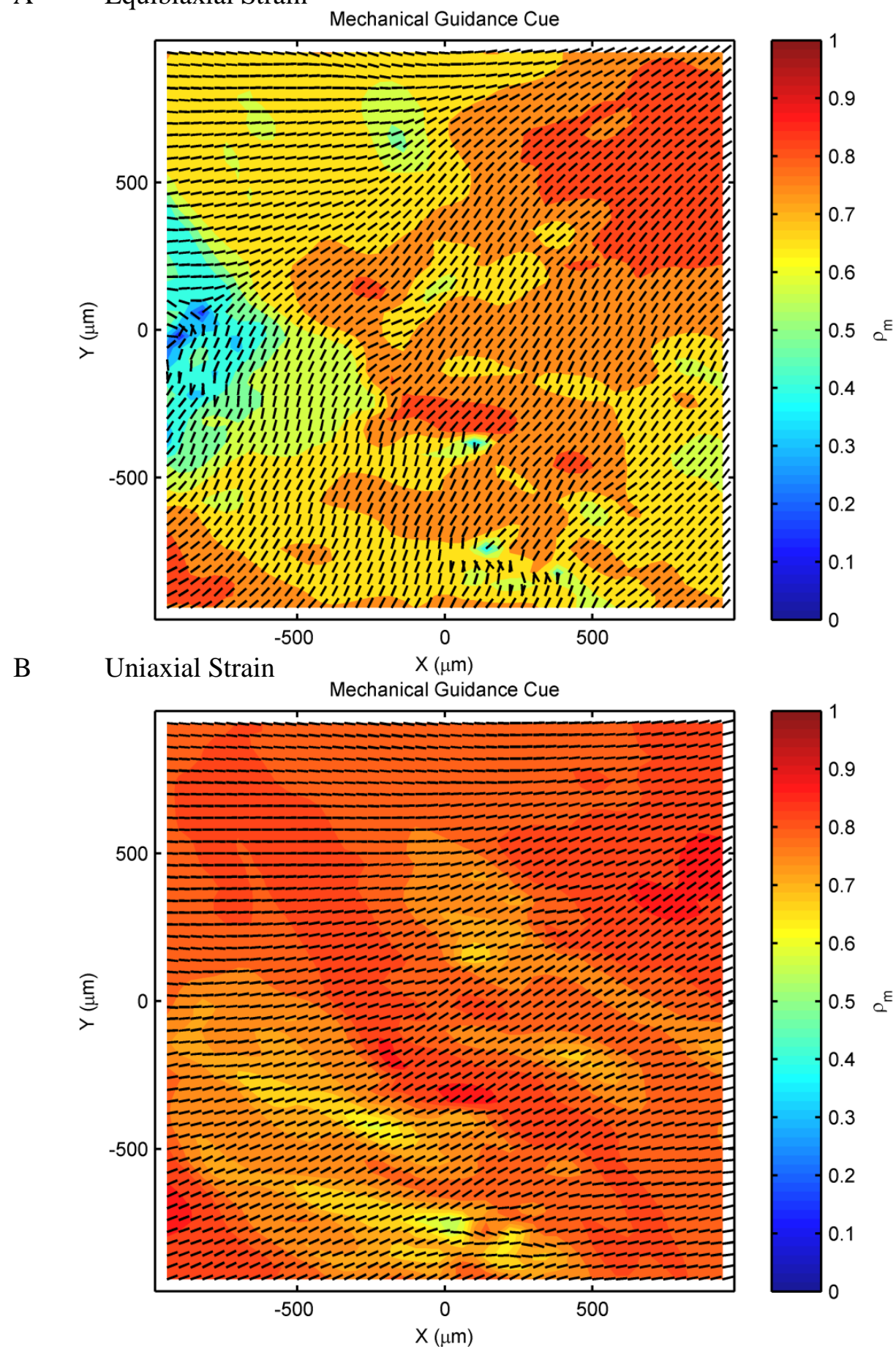

Figure 6.10. Effect of spatial heterogeneity of fiber angles on mechanical guidance cues. We simulated equibiaxial stretch (A) or uniaxial stretch $(\mathbf{B})$ of a $2 \mathrm{~mm}$ by $2 \mathrm{~mm}$ sheet of 3 week old cryoinfarct scar with a realistic spatial arrangement of fiber angles (Fig. 6.6) and calculated the expected strength of cell alignment $\left(\rho_{m}\right)$ and axis of cell alignment (dashes in the plots) stimulated by the mechanical guidance cue (anisotropic strain) at each element. A. For equibiaxial stretch, the spatially heterogeneous fiber angles were predicted to cause spatial heterogeity of the strengths and axes of cell alignment. The axis of cell alignment was generally perpendicular or nearly perpendicular to the local fiber axis (compare to Fig. 6.6). B. For uniaxial stretch, the strengths and axes of cell alignment displayed less spatial heterogeneity due to the globally anisotropic strain pattern. 


\subsection{REFERENCES}

Arts T, Lumens J, Kroon W \& Delhaas T (2012). Control of whole heart geometry by intramyocardial mechano-feedback: a model study. PLoS Computational Biology 8, e1002369.

Bujak M \& Frangogiannis NG (2007). The role of TGF-beta signaling in myocardial infarction and cardiac remodeling. Cardiovascular Research 74, 184-195.

Costa KD, Holmes JW \& McCulloch AD (2001). Modelling cardiac mechanical properties in three dimensions. Philosophical Transactions of the Royal Society A: Mathematical, Physical and Engineering Sciences 359, 1233-1250.

Dewald O, Zymek P, Winkelmann K, Koerting A, Ren G, Abou-Khamis T, Michael LH, Rollins BJ, Entman ML \& Frangogiannis NG (2005). CCL2/monocyte chemoattractant protein-1 regulates inflammatory responses critical to healing myocardial infarcts. Circulation Research 96, 881-889.

Fomovsky GM \& Holmes JW (2010). Evolution of scar structure, mechanics, and ventricular function after myocardial infarction in the rat. American Journal of Physiology: Heart and Circulatory Physiology 298, H221-H228.

Fomovsky GM, Rouillard AD \& Holmes JW (2012). Regional mechanics determine collagen fiber structure in healing myocardial infarcts. Journal of Molecular and Cellular Cardiology 52, 1083-1090.

Frangogiannis NG (2008). The immune system and cardiac repair. Pharmacological Research 58, 88-111.

Gallagher KP, Gerren RA, Stirling MC, Choy M, Dysko RC, McManimon SP \& Dunham WR (1986). The distribution of functional impairment across the lateral border of acutely ischemic myocardium. Circulation Research 58, 570-583.

Holmes JW (2004). Candidate mechanical stimuli for hypertrophy during volume overload. Journal of Applied Physiology 97, 1453-1460.

Holmes JW, Borg TK \& Covell JW (2005). Structure and mechanics of healing myocardial infarcts. Annual Review of Biomedical Engineering 7, 223-253.

Holmes JW, Nuñez JA \& Covell JW (1997). Functional implications of myocardial scar structure. American Journal of Physiology: Heart and Circulatory Physiology 272, H2123H2130.

Jin Y, Han H, Berger J, Dai Q \& Lindsey ML (2011). Combining experimental and mathematical modeling to reveal mechanisms of macrophage-dependent left ventricular remodeling. BMC Systems Biology 5, 1-14. 
Karlon WJ, Hsu PP, Li S, Chien S, McCulloch AD \& Omens JH (1999). Measurement of orientation and distribution of cellular alignment and cytoskeletal organization. Annals of Biomedical Engineering 27, 712-720.

Kerckhoffs RCP, Omens J \& McCulloch AD (2012). A single strain-based growth law predicts concentric and eccentric cardiac growth during pressure and volume overload. Mechanics Research Communications 42, 40-50.

Kroon W, Delhaas T, Bovendeerd P \& Arts T (2008). Structure and torsion in the normal and situs inversus totalis cardiac left ventricle. II. modeling cardiac adaptation to mechanical load. American Journal of Physiology Heart and Circulatory Physiology 295, H202-H210.

Lindsey ML \& Zamilpa R (2012). Temporal and spatial expression of matrix metalloproteinases and tissue inhibitors of metalloproteinases following myocardial infarction. Cardiovascular Therapeutics 30, 31-41.

Mazhari R, Omens JH, Covell JW \& McCulloch AD (2000). Structural basis of regional dysfunction in acutely ischemic myocardium. Cardiovascular Research 47, 284-293.

McCurdy SM, Dai Q, Zhang J, Zamilpa R, Ramirez TA, Dayah T, Nguyen N, Jin Y-F, Bradshaw AD \& Lindsey ML (2011). SPARC mediates early extracellular matrix remodeling following myocardial infarction. American Journal of Physiology Heart and Circulatory Physiology 301, H497-H505.

Mitchell MD, Laird RE, Brown RD \& Long CS (2007). IL-1beta stimulates rat cardiac fibroblast migration via MAP kinase pathways. American Journal of Physiology Heart and Circulatory Physiology 292, H1139-H1147.

Rane AA \& Christman KL (2011). Biomaterials for the treatment of myocardial infarction: a 5year update. Journal of the American College of Cardiology 58, 2615-2629.

Rosenkranz S (2004). TGF-beta1 and angiotensin networking in cardiac remodeling. Cardiovascular Research 63, 423-432.

Rouillard AD \& Holmes JW (2012). Mechanical regulation of fibroblast migration and collagen remodelling in healing myocardial infarcts. The Journal of Physiology 590, 4585-4602.

Streeter DD \& Hanna WT (1973). Engineering mechanics for successive states in canine left ventricular myocardium. Circulation Research 33, 639-655.

Zahedmanesh H \& Lally C (2012). A multiscale mechanobiological modelling framework using agent-based models and finite element analysis: application to vascular tissue engineering. Biomechanics and Modeling in Mechanobiology 11, 363-377. 


\section{CHAPTER 7: Summary and Future Directions}

\subsection{MOTIVATION}

The overall goal of this work was to understand the regulation of collagen fiber alignment during healing after myocardial infarction. The collagen fiber structure of infarct scar tissue largely determines its mechanical properties (Holmes et al., 2005). In turn, the mechanical properties of infarct scar tissue affect heart function (Laird \& Vellekoop, 1977). Because soft infarcts bulge during systole and waste energy that would otherwise aid blood ejection and stiff infarcts resist expansion during diastole and impair filling of the heart (Holmes et al., 2005), our lab has been interested in the possibility that anisotropic infarcts support better heart function than isotropic infarcts (Holmes et al., 1997; Fomovsky et al., 2011, 2012a). While other work in our lab has focused on the relationship between infarct scar anisotropy and heart function, this work focused on factors that regulate infarct scar anisotropy (Rouillard \& Holmes, 2012). By understanding the factors that determine whether collagen fibers are organized into isotropic or anisotropic structures, we may be able to control infarct scar anisotropy for experimental investigation of its impact on heart function and possibly for therapeutic benefit. Because a myriad of potential therapies for myocardial infarction are currently in development (Johnston et al., 2009; Chung et al., 2010; Morita et al., 2011; Rane \& Christman, 2011), we may also be able to anticipate unintended impacts of such interventions on the collagen fiber structure, and in turn, mechanical properties, of infarct scar. 


\subsection{APPROACH}

Our overall approach was to build a computational model of infarct healing and use it to test hypotheses about how collagen fiber alignment is regulated during infarct healing. This approach relied on inference from experimental data. Hypotheses were converted into alternative versions of the computational model. For example, we built a model that turned off the alignment response of fibroblasts to uniaxial strain in order to test the hypothesis that a mechanical guidance cue is necessary to explain collagen fiber alignment observed in infarct scars. For each hypothesis (candidate model), we simulated infarct healing and tested if the model reproduced experimentally measured features of infarct scar, such as the strengths of cell and collagen fiber alignment. Hypotheses that yielded model predictions that agreed with experimental data were considered more likely to be true than hypotheses that yielded model predictions that disagreed with experimental data.

We focused our efforts on understanding the behavior of fibroblasts, the cells primarily responsible for depositing and remodeling the collagen fibers of infarct scar matrices (Kanekar et al., 1998; Porter \& Turner, 2009; Souders et al., 2009). Our hypotheses revolved around the question of how the collagen fiber structure of infarct scar arises from the collective activity of individual fibroblasts sensing and responding to signals in their local environment. We decided to build an agent-based model, because, as the name implies, agent-based models are well-suited to simulating individual actions of many agents (e.g. fibroblasts) (Mi et al., 2007; Chavali et al., 2008; Hashambhoy et al., 2011). At the heart of our agent-based model of infarct healing was a set of instructions that dictated the actions of fibroblasts (limited to actions relevant to collagen 
matrix synthesis and remodeling) conditional upon the local environment of each fibroblast. The instructions were written as mathematical equations. For example, the rate at which fibroblasts deposit collagen fibers was written as a function of the local concentration of growth factors. Essentially, the objective of this work was to assemble and explain the consequences of an evidence-based set of instructions (a model) that embodies our understanding of the regulation of collagen fiber alignment during infarct healing.

\subsection{CHAPTER SUMMARIES}

Chapter 2: Mechanical Regulation of Fibroblast Migration and Collagen Remodeling in Healing Myocardial Infarcts

First, we tested the hypothesis that a mechanical guidance cue is necessary to explain collagen fiber alignment observed in infarct scars. Prior studies have suggested that mechanical, structural, or chemical cues could regulate the anisotropy of infarct scar tissue (Zimmerman et al., 2000; McDougall et al., 2006; Fomovsky et al., 2012b). We developed an agent-based model that accounted for the combined influence of these cues on fibroblast alignment, collagen deposition, and collagen remodeling (Rouillard \& Holmes, 2012). We pooled published experimental data from several sources in order to determine parameter values in the model, and then tested model predictions against collagen alignment measurements from a set of experiments that measured collagen fiber alignment in cryoinfarcts that were exposed to different patterns of stretch during healing (Fomovsky et al., 2012b). We independently perturbed the mechanical, structural, and chemical cues in the model in order to determine how 
strongly each cue influenced the predicted collagen structure. Although chemokine gradients and pre-existing matrix structures influenced spatial and temporal patterns of collagen organization in certain situations, a mechanical cue was critical to reproducing the collagen alignment observed experimentally in healing infarcts.

\section{Chapter 3: Computational Screening of Candidate Processes of Collagen Fiber Remodeling in Healing Myocardial Infarcts}

Next, we tested the hypothesis that active re-orientation of nearby collagen fibers by fibroblasts is necessary to explain collagen fiber alignment observed in infarct scars. In vitro studies have shown that mechanical cues can cause collagen fiber alignment 1 . by guiding alignment of fibroblasts, which then deposit collagen fibers aligned with the current cell orientation (aligned deposition) (Wang et al., 2003; Kapacee et al., 2008); 2. by guiding alignment of fibroblasts, which then generate traction forces that cause rotation of nearby collagen fibers toward alignment with the current cell orientation (rotation) (Petroll et al., 2003; Lee et al., 2008); or 3 . by reducing the rate of degradation of collagen fibers under tension, which promotes accumulation of collagen fibers aligned along the axis of tension (selective degradation) (Huang \& Yannas, 1977; Flynn et al., 2010). We modified our agent-based model to allow collagen fiber remodeling via aligned deposition only, rotation only, or selective degradation only and tested how well each model could reproduce the collagen alignment and cell alignment observed in healing infarcts. The aligned deposition model and rotation model were able to reproduce the experimental data equally well, whereas the selective degradation model under-predicted the strength of collagen alignment. We performed parameter sensitivity 
analyses on the aligned deposition and rotation models and discovered that they predicted different sensitivities of the strength of collagen alignment to the rates of collagen generation and degradation. We demonstrated that experiments that perturb these rates during infarct healing and measure collagen fiber alignment could provide data to further test whether collagen fiber remodeling during infarct healing is mediated by aligned deposition or rotation.

\section{Chapter 4: Empirical Approach to Predicting Cell Alignment and Biased Migration Driven by Mechanical, Structural, and Simultaneous Guidance Cues}

\section{Next, we developed an empirical approach to predicting fibroblast alignment and biased migration in response to environmental guidance cues. Prior studies have modeled the} influence of guidance cues on the orientation of a cell by representing guidance cues as vectors, computing the weighted sum of these vectors, and defining the cell orientation as the orientation of the resultant vector (McDougall et al., 2006). In our agent-based model, we modified this approach by using the resultant vector to define a probability distribution of cell orientations, in order to account for observations that guidance cues bias cell orientations but rarely dictate orientations absolutely. This approach worked well for predicting the cell and collagen fiber alignment observed in healing infarcts, but some of the parameter values and equations we used to compute and combine the guidance cue vectors were not firmly grounded in experimental evidence. We built an empirical foundation for this approach by formulating equations and fitting for parameter values that could reproduce experimental measurements-reported in the literature — of cell alignment in response to mechanical (cyclic uniaxial stretch), structural (micro-ridged substrates), or simultaneous mechanical and structural guidance cues. The 
literature data supported our general approach to representing guidance cues as vectors, combining guidance cues as a weighted vector sum, and relating the resultant vector to a cell orientation probability distribution. Furthermore, the literature data supported our prior conclusions about the roles of structural and mechanical cues during infarct healing. When we updated the agent-based model with the new equations and parameter values based on the in vitro data, the predicted strengths and weightings of the structural and mechanical guidance cues were similar to the values we estimated previously, and the results of the infarct healing simulations were nearly unchanged. We do not yet have a complete understanding of how guidance cues will interact in all situations, but we expect more progress can be made with further experimental investigation of cyclic stretch applied to cells plated on micro-textured substrates.

Chapter 5: Mechanical Regulation of Fibroblast Migration in a Collagen-Fibrin Wound Model

Next, we tested the hypothesis that anisotropic mechanical boundary conditions increase the directionality and speed of migration of fibroblasts into a model wound. Many in vitro studies have shown that fibroblast behaviors such as proliferation (Berry et al., 2003), matrix synthesis (Atance et al., 2004), matrix degradation (Tyagi et al., 1998), and alignment (Katsumi et al., 2004; Lee et al., 2008; Pang et al., 2011) are sensitive to mechanical stimuli. Extending our previous analysis, which suggested that mechanical guidance cues strongly affect fibroblast alignment during infarct healing, we tested if mechanical guidance cues also affect fibroblast migration during wound healing, which simultaneously exposes fibroblasts to competing 
structural and chemical guidance cues. We created elliptical fibrin "wounds" in fibroblastpopulated collagen gels and applied uniaxial mechanical restraint at the edges of the collagen gels along the short or long axis of the fibrin wounds. We measured apparent orientations and apparent speeds of migrating fibroblasts and found a strong guidance effect of short axis restraint relative to long axis restraint, without an increase in speed of fibroblast migration along the short axis of the wounds. We adapted our approach to calculating cell orientation probabilities - in this case, calculating orientation probabilities of cell displacements - in order to infer what guidance cues could explain the migration behavior that we observed. The different migration behavior between long axis restraint and short axis restraint could be explained as a response to different mechanical guidance cues.

\section{Chapter 6: Agent-Based Model with Integrated Finite Element Analysis for Investigation of the Mechanobiology of Healing Myocardial Infarcts}

Finally, we developed an approach to coupling a finite element model to the agent-based model of infarct healing in order to predict how strains, and therefore mechanical guidance cues, change as infarct structure evolves. Ultimately, in order to predict how interventions that change the collagen fiber structure of infarct scar affect heart function, we must first compute the mechanical properties of the scar, and then use a finite element model of the heart to compute how the presence of a scar with those mechanical properties affects heart function (Fomovsky et al., 2011). This task is complicated by the fact that infarct strains, and therefore mechanical guidance cues, are partly determined by the structural properties of the infarct. We anticipated a feedback loop, where the pattern of mechanical strain guides fibroblast and 
collagen fiber alignment, which changes the structural properties of the infarct, which changes the infarct strains. We developed a finite element model of an infarct and surrounding myocardium and developed an approach to coupling the finite element model to our agent-based model of infarct healing. We defined coefficients of the constitutive equation of the finite element model as functions of the collagen area fraction and collagen mean vector length predicted by the agent-based model, and computed mechanical guidance cues in the agent-based model from strains predicted by the finite element model. Preliminary analysis suggested that infarct strains, and therefore mechanical guidance cues, are insensitive to changes in collagen area fraction and collagen mean vector length that fall within the range of observed variations. We also predicted that strains in a scar with a spatially heterogeneous collagen fiber structure should create mechanical guidance cues that act to eliminate, rather than support, the structural heterogeneity. Further study of the co-evolution of the structure and mechanics of infarct scar requires improvement of the finite element model and experimental data supporting the method of computing constitutive properties from the collagen fiber distribution of infarct scar.

\subsection{FUTURE DIRECTIONS}

The overall goal of this work was to understand the regulation of collagen fiber alignment during healing after myocardial infarction. We developed a computational model of infarct healing that explains many aspects of how environmental cues influence the behavior of fibroblasts, the cells primarily responsible for depositing and remodeling the collagen fiber matrix of infarct scar. We have also identified several key directions for future work. In Chapter 2, we noted that measurements of the structure of collagen fibers (and other matrix fibers) during the first week 
after infarction are lacking, but would potentially be of high value given the dramatic thinning and expansion of the infarct that leads to impaired heart function and risk of rupture early after infarction (Whittaker et al., 1991; Gaudron et al., 1993; Gao et al., 2005, 2012). In Chapter 3, we designed experiments that should help us determine if collagen fiber remodeling during healing after infarction occurs predominantly via aligned deposition or fiber rotation. In Chapter 4, we emphasized the need for more experimental data studying the alignment of cells in response to simultaneous guidance cues of varying magnitudes and orientations. In Chapter 6, we introduced a significant advancement of the computational model, a coupled FEM-ABM, which could ultimately link predictions of infarct structure to predictions of heart function, but needs further model development and more experimental data from which to derive a structurebased constitutive equation.

More broadly, as our understanding of the regulation of fibroblasts and collagen fibers improves, we should consider interactions with other cell types and matrix components, especially for study of healing during the first week or two after infarction. For example, inflammatory cells such as macrophages and neutrophils degrade matrix and necrotic muscle and secrete growth factors that regulate fibroblast behavior (Frangogiannis et al., 2002), and fibrin and fibronectin form a provisional matrix prior to collagen fiber deposition (Dobaczewski et al., 2006). Early after infarction, these cell types and matrix fibers may be more important determinants of infarct structure and mechanics than fibroblasts and collagen fibers. We should also look toward ways in which the model can improve efforts to develop therapies for myocardial infarction. For example, we may be able to use our understanding of how environmental guidance cues regulate 
cell and matrix alignment to design strategies for guiding structural integration of regenerated myocardium (once achieved) with surrounding native myocardium. 


\subsection{REFERENCES}

Atance J, Yost MJ \& Carver W (2004). Influence of the extracellular matrix on the regulation of cardiac fibroblast behavior by mechanical stretch. Journal of Cellular Physiology 200, 377386.

Berry CC, Shelton JC, Bader DL \& Lee DA (2003). Influence of external uniaxial cyclic strain on oriented fibroblast-seeded collagen gels. Tissue Engineering 9, 613-624.

Chavali AK, Gianchandani EP, Tung KS, Lawrence MB, Peirce SM \& Papin JA (2008). Characterizing emergent properties of immunological systems with multi-cellular rulebased computational modeling. Trends in Immunology 29, 589-599.

Chung ES, Dan D, Solomon SD, Bank AJ, Pastore J, Iyer A, Berger RD, Franklin JO, Jones G, Machado C \& Stolen CM (2010). Effect of peri-infarct pacing early after myocardial infarction: results of the prevention of myocardial enlargement and dilatation post myocardial infarction study. Circulation Heart Failure 3, 650-658.

Dobaczewski M, Bujak M, Zymek P, Ren G, Entman ML \& Frangogiannis NG (2006). Extracellular matrix remodeling in canine and mouse myocardial infarcts. Cell and Tissue Research 324, 475-488.

Flynn BP, Bhole AP, Saeidi N, Liles M, DiMarzio CA \& Ruberti JW (2010). Mechanical strain stabilizes reconstituted collagen fibrils against enzymatic degradation by mammalian collagenase matrix metalloproteinase 8 (MMP-8). PLoS ONE 5, e12337.

Fomovsky GM, Clark SA, Parker KM, Ailawadi G \& Holmes JW (2012a). Anisotropic reinforcement of acute anteroapical infarcts improves pump function. Circulation Heart Failure 5, 515-522.

Fomovsky GM, Macadangdang JR, Ailawadi G \& Holmes JW (2011). Model-based design of mechanical therapies for myocardial infarction. Journal of Cardiovascular Translational Research 4, 82-91.

Fomovsky GM, Rouillard AD \& Holmes JW (2012b). Regional mechanics determine collagen fiber structure in healing myocardial infarcts. Journal of Molecular and Cellular Cardiology 52, 1083-1090.

Frangogiannis NG, Smith CW \& Entman ML (2002). The inflammatory response in myocardial infarction. Cardiovascular research 53,31-47.

Gao X-M, White DA, Dart AM \& Du X-J (2012). Post-infarct cardiac rupture: recent insights on pathogenesis and therapeutic interventions. Pharmacology \& Therapeutics 134, 156-179. 
Gao X-M, Xu Q, Kiriazis H, Dart AM \& Du X-J (2005). Mouse model of post-infarct ventricular rupture: time course, strain- and gender-dependency, tensile strength, and histopathology. Cardiovascular Research 65, 469-477.

Gaudron P, Eilles C, Kugler I \& Ertl G (1993). Progressive left ventricular dysfunction and remodeling after myocardial infarction. Circulation 87, 755-763.

Hashambhoy YL, Chappell JC, Peirce SM, Bautch VL \& Mac Gabhann F (2011). Computational modeling of interacting VEGF and soluble VEGF receptor concentration gradients. Frontiers in Physiology 2, 62.

Holmes JW, Borg TK \& Covell JW (2005). Structure and mechanics of healing myocardial infarcts. Annual Review of Biomedical Engineering 7, 223-253.

Holmes JW, Nuñez JA \& Covell JW (1997). Functional implications of myocardial scar structure. American Journal of Physiology: Heart and Circulatory Physiology 272, H2123$\mathrm{H} 2130$.

Huang C \& Yannas I (1977). Mechanochemical studies of enzymatic degradation of insoluble collagen fibers. Journal of Biomedical Materials Research 8, 137-154.

Johnston P V, Sasano T, Mills K, Evers R, Lee S-T, Smith RR, Lardo AC, Lai S, Steenbergen C, Gerstenblith G, Lange R \& Marbán E (2009). Engraftment, differentiation, and functional benefits of autologous cardiosphere-derived cells in porcine ischemic cardiomyopathy. Circulation 120, 1075-1083.

Kanekar S, Hirozanne T, Terracio L \& Borg TK (1998). Cardiac fibroblasts: form and function. Cardiovascular Pathology 7, 127-133.

Kapacee Z, Richardson SH, Lu Y, Starborg T, Holmes DF, Baar K \& Kadler KE (2008). Tension is required for fibripositor formation. Matrix Biology 27, 371-375.

Katsumi A, Orr a W, Tzima E \& Schwartz MA (2004). Integrins in mechanotransduction. The Journal of biological chemistry 279, 12001-12004.

Laird JD \& Vellekoop HP (1977). Time course of passive elasticity of myocardial tissue following experimental infarction in rabbits and its relation to mechanical dysfunction. Circulation Research 41, 715-721.

Lee EJ, Holmes JW \& Costa KD (2008). Remodeling of engineered tissue anisotropy in response to altered loading conditions. Annals of Biomedical Engineering 36, 1322-1334.

McDougall S, Dallon J, Sherratt J \& Maini P (2006). Fibroblast migration and collagen deposition during dermal wound healing: mathematical modelling and clinical implications. Philosophical Transactions of the Royal Society A: Mathematical, Physical and Engineering Sciences 364, 1385-1405. 
Mi Q, Rivière B, Clermont G, Steed DL \& Vodovotz Y (2007). Agent-based model of inflammation and wound healing: insights into diabetic foot ulcer pathology and the role of transforming growth factor-beta1. Wound Repair and Regeneration 15, 671-682.

Morita M, Eckert CE, Matsuzaki K, Noma M, Ryan LP, Burdick JA, Jackson BM, Gorman JH, Sacks MS \& Gorman RC (2011). Modification of infarct material properties limits adverse ventricular remodeling. Annals of Thoracic Surgery 92, 617-625.

Pang Y, Wang X, Lee D \& Greisler HP (2011). Dynamic quantitative visualization of single cell alignment and migration and matrix remodeling in 3-D collagen hydrogels under mechanical force. Biomaterials 32, 3776-3783.

Petroll WM, Ma L \& Jester J V (2003). Direct correlation of collagen matrix deformation with focal adhesion dynamics in living corneal fibroblasts. Journal of Cell Science 116, 14811491.

Porter KE \& Turner NA (2009). Cardiac fibroblasts: at the heart of myocardial remodeling. Pharmacology \& Therapeutics 123, 255-278.

Rane AA \& Christman KL (2011). Biomaterials for the treatment of myocardial infarction: a 5year update. Journal of the American College of Cardiology 58, 2615-2629.

Rouillard AD \& Holmes JW (2012). Mechanical regulation of fibroblast migration and collagen remodelling in healing myocardial infarcts. The Journal of Physiology 590, 4585-4602.

Souders CA, Bowers SLK \& Baudino TA (2009). Cardiac fibroblast: the renaissance cell. Circulation Research 105, 1164-1176.

Tyagi SC, Lewis K, Pikes D, Marcello A, Mujumdar VS, Smiley LM \& Moore CK (1998). Stretch-induced membrane type matrix metalloproteinase and tissue plasminogen activator in cardiac fibroblast cells. Journal of Cellular Physiology 176, 374-382.

Wang JH-C, Jia F, Gilbert TW \& Woo SL-Y (2003). Cell orientation determines the alignment of cell-produced collagenous matrix. Journal of Biomechanics 36, 97-102.

Whittaker P, Boughner DR \& Kloner RA (1991). Role of collagen in acute myocardial infarct expansion. Circulation 84, 2123-2134.

Zimmerman SD, Karlon WJ, Holmes JW, Omens JH \& Covell JW (2000). Structural and mechanical factors influencing infarct scar collagen organization. American Journal of Physiology Heart and Circulatory Physiology 278, H194-200. 